\title{
Molecular physiology of synaptic sound encoding at the first auditory synapse
}

\author{
Dissertation \\ for the award of the degree \\ "Doctor rerum naturalium" \\ of the Georg-August-Universität Göttingen \\ within the doctoral program "Molecular Biology" \\ of the Georg-August University School of Science (GAUSS)
}

\author{
Submitted by \\ Stefanie Krinner \\ Born in \\ Munich, Germany
}

Göttingen 2017 


\section{Members of the Thesis Committee}

\section{Prof. Dr. Tobias Moser}

Institute for Auditory Neuroscience \& InnerEarLab, University Medical Center Göttingen

Prof. Dr. Erwin Neher

Department of Membrane Biophysics, Max-Planck-Institute for Biophysical Chemistry

Prof. Dr. Stefan Hell

Department of NanoBiophotonics, Max-Planck-Institute for Biophysical Chemistry

\section{Further members of the Examination Board}

\section{Prof. Dr. Nils Brose}

Department of Molecular Neurobiology, Max-Planck-Institute of Experimental Medicine, Göttingen

\section{Prof. Dr. Thomas Dresbach}

Department of Anatomy and Embryology, Georg-August University, Göttingen

\section{Dr. Manuela Schmidt}

Somatosensory Signaling and Systems Biology, Max-Planck-Institute of Experimental Medicine, Göttingen

Date of oral examination: November $22^{\text {nd }}, 2017$ 


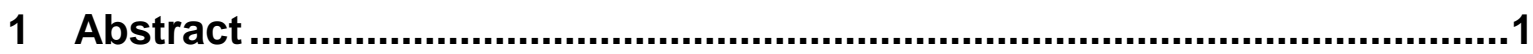

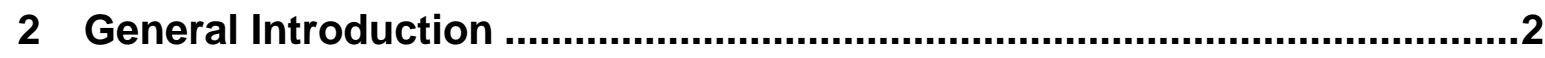

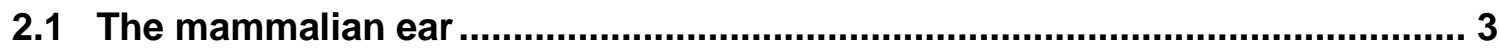

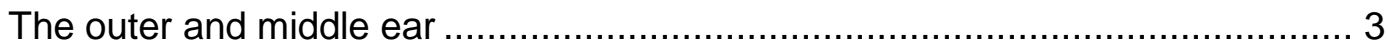

The inner ear and organ of Corti ............................................................. 4

2.2 Inner hair cell ribbon synapses .................................................................... 8

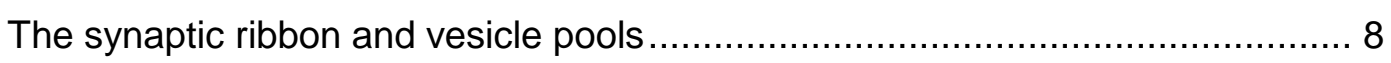

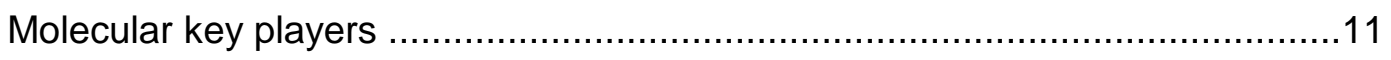

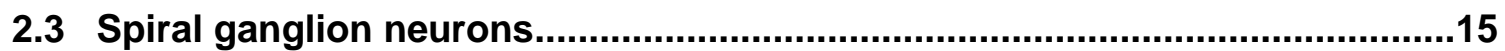

Cochlear innervation pattern and spike generation........................................15

Heterogeneous response characteristics ................................................16

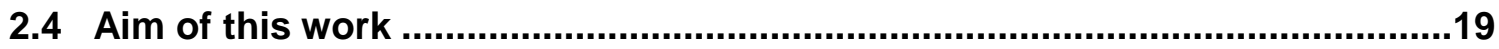

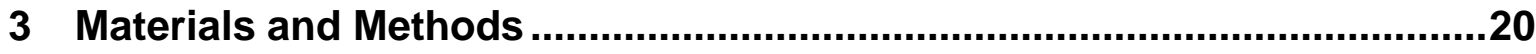

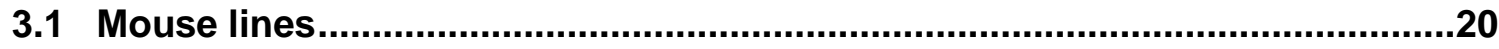

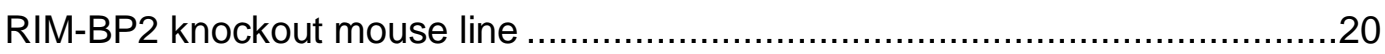

Floxpatch and Bhlhb5-Cre mouse line.................................................20

3.2 Viral optopatch construct and postnatal injection of AAV ...........................21

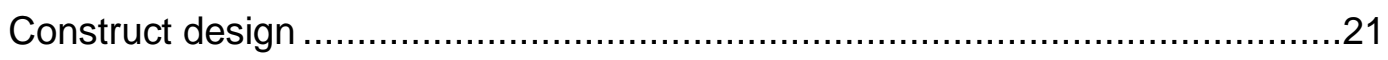

Postnatal injection of AAV ..............................................................22

3.3 Whole-mount preparations of the organ of Corti ..........................................23

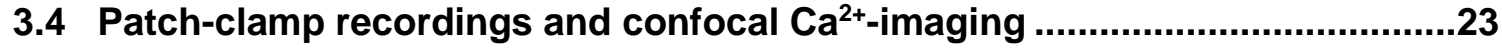

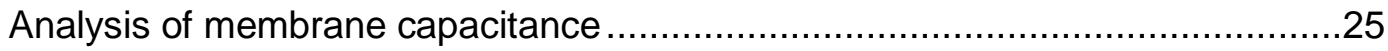

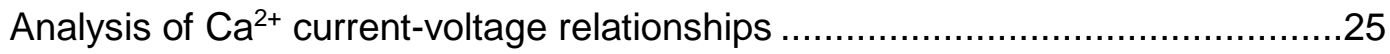

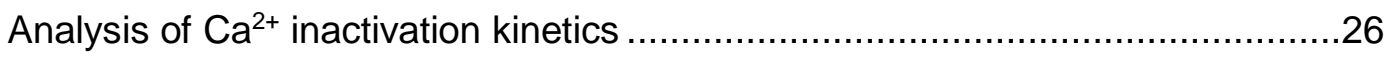

Estimating the apparent $\mathrm{Ca}^{2+}$ cooperativity of exocytosis ..............................26

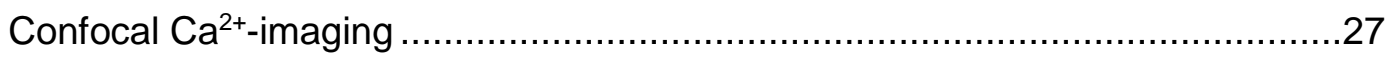

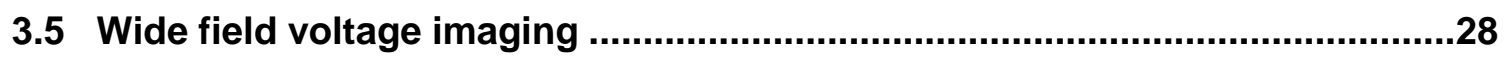

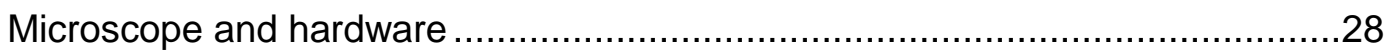

Optogenetic and patch-clamp stimulation..................................................29

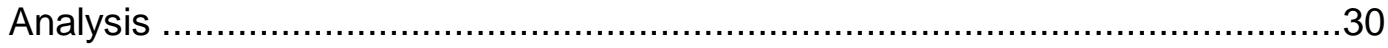

3.6 Immunohistochemistry, confocal and STED microscopy .................................30

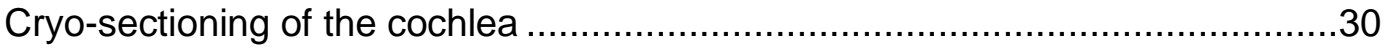

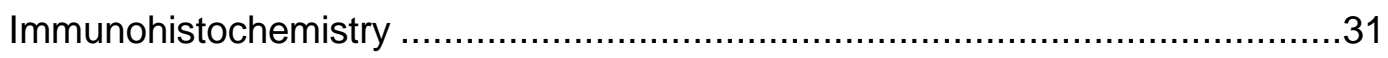

Fluorescence microscopy and data analysis ............................................32

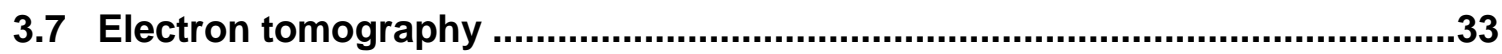

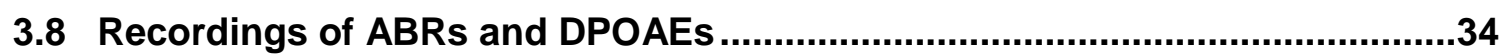




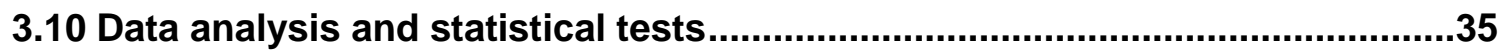

4 Chapter 1 .36

"RIM-binding protein 2 promotes the number of voltage-gated Cav1.3 $\mathrm{Ca}^{2+}$-channels at hair cell active zones and contributes to fast synaptic vesicle recruitment"

4.1 Introduction .37

4.2 Results .39

RIM-BP2 forms (double-) stripe shaped clusters at IHC AZs .................................... 39

RIM-BP2 promotes presynaptic $\mathrm{Ca}^{2+}$-influx in IHCs ............................................. 41

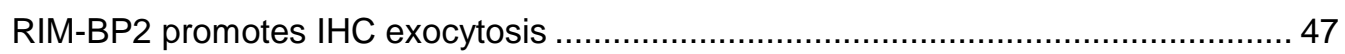

RIM-BP2 does not regulate $\mathrm{Ca}^{2+-i n f l u x}$ - exocytosis coupling of RRP SVs in IHCs ... 50

RIM-BP2 facilitates fast SV recovery after RRP depletion ..................................... 52

RIM-BP2 regulates the distance of SVs to the presynaptic IHC membrane ............... 53

RIM-BP2 disruption causes a mild impairment of synaptic sound encoding ............... 57

4.3 Discussion. .63

Impact of RIM-BP2 on synaptic $\mathrm{Ca}^{2+}$-channel abundance and their coupling to SVs. .64

RIM-BP2 regulates the abundance of L-type $\mathrm{Ca}^{2+-}$-channels at ribbon synapses ....... 64

Potential impact of RIM-BP2 - bassoon interaction ............................................. 66

Potential compensatory recruitment of N-type $\mathrm{Ca}^{2+-c h a n n e l s ~ u p o n ~ l o s s ~ o f ~ R I M-B P 2 ~} 66$

Tight $\mathrm{Ca}^{2+}$ nanodomain-like coupling at IHC ribbon synapses . .67

Potential interaction partners of RIM-BP2 at IHCs.......................................69

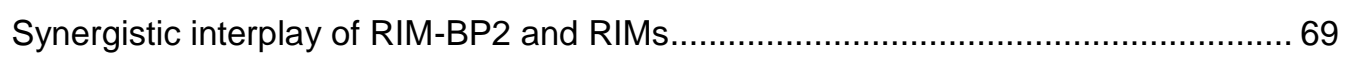

Potential impact on RIM-BP2 - CAST/ERC (ELKS) interaction ................................ 70

Impact of RIM-BP2 on sound encoding at the IHC ribbon synapse ....................71

Potential mechanism of RIM-BP2 function during vesicle replenishment ................... 71

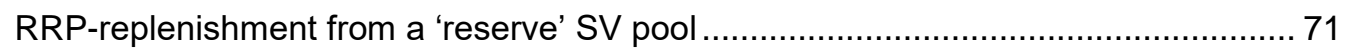

Potential molecular mechanism of RIM-BP2 at IHC AZs ...............................75

5 Chapter 2 .........................................................................................77

"Establishing voltage imaging in spiral ganglion neurons"

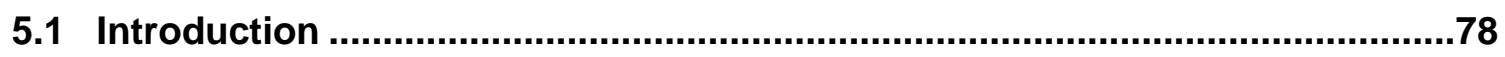

5.2 Results .80

Expression of QuasAr2 and all-optical electrophysiology in the cochlea of Floxpatch-

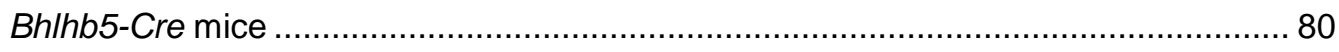

Expression of QuasAr3 in the postnatally virus-transduced mouse cochlea.............. 83

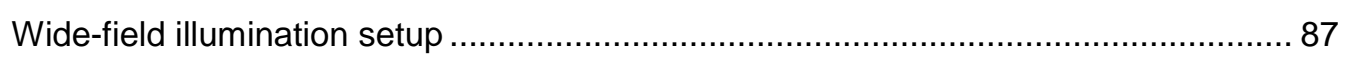

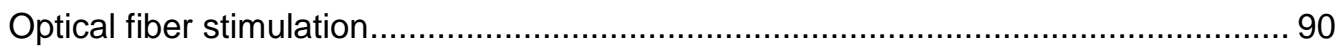

All-optical electrophysiology in AAV2/6-Optopatch3 transduced mouse cochleae ..... 93 
5.3 Discussion

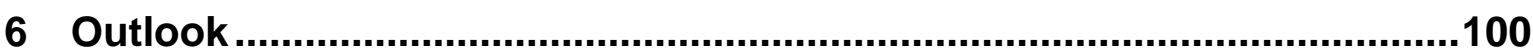

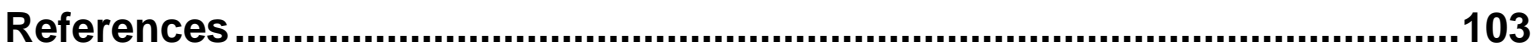

List of abbreviations .................................................................................116

Acknowledgements.............................................................................118

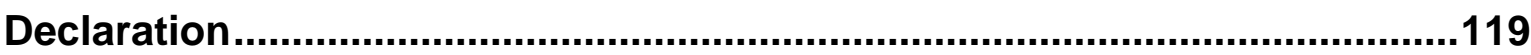

Curriculum Vitae............................................................................................120 


\section{Abstract}

To achieve accurate encoding of sounds, inner hair cell (IHC) ribbon-type synapses are highly specialized to release synaptic vesicles (SVs) with high rates and temporal precision. A sophisticated, by far not yet fully disentangled unconventional molecular machinery at the synapse active zone (AZ) realizes this impressive performance. It regulates the number of synaptic Cav1.3 $\mathrm{Ca}^{2+}$-channels, their tight coupling to SVs, and fast re-supply of SVs for sustained rates of exocytosis. Sound-evoked glutamate release from an IHC synapse is sensed by the postsynaptic spiral ganglion neurons (SGNs). Each SGN is innervated by only one ribbon-type synapse. Even though one might expect similar response characteristics from SGNs innervating the same IHC, this is surprisingly not the case. Postsynaptic spike responses of SGNs differ remarkably, which is likely used as a presynaptic mechanism to encode sounds of varying intensity. Recently, a positive correlation between different SGN response types and presynaptic synapse properties has been found. However, a functional link between heterogeneous presynaptic properties and postsynaptic SGN spike response diversity remains to be demonstrated. To draw a clearer picture of the synaptic transmission mechanism in IHCs, it is key to characterize the molecular components. Furthermore, it is critical to understand the coding strategies of sensory IHCs that specialize and fine-tune the synapses to mediate sound coding. In this work, I addressed these questions in two different approaches. (1) First, the molecular physiology of synaptic transmission at the IHC ribbon synapse was investigated by examining the role of RIM-binding protein 2 (RIM-BP2), a multidomain cytomatrix protein acting as molecular hub between $\mathrm{Ca}^{2+}$-channels and vesicular release sites. $\mathrm{A}$ multidisciplinary approach including confocal and STED immunofluorescence microscopy, electron microscopy, patch-clamp, and confocal $\mathrm{Ca}^{2+}$-imaging, as well as auditory systems physiology was utilized to explore the morphological and physiological effects of genetic RIM-BP2 disruption in constitutive RIM-BP2 knockout mice. I found evidence that RIM-BP2 positively regulates the number of synaptic Cav1.3 $\mathrm{Ca}^{2+}$-channels and thereby facilitates SV release and enhances fast SV recruitment after RRP depletion. Furthermore, recordings of auditory brainstem responses (ABRs) and of single auditory nerve fibers (ANFs) showed a mild deficit of sound encoding. (2) Second, an experimental setup for voltage imaging in SGNs was established, to simultaneously monitor multiple SGN responses innervating the same IHC and thereby create a system to understand the synaptic coding strategies of IHCs. The genetically encoded voltage indicators (GEVIs) QuasAr2 and 3 were specifically targeted to SGNs, however only QuasAr3 elicited fluorescence responses in SGN boutons adjacent to an IHC. Thus, henceforth QuasAr3 might be a suitable tool to probe the presynaptic mechanism of postsynaptic response diversity. 


\section{General Introduction}

A sense is the physiological capacity of an organism to perceive information from the outside world. Humans possess a multitude of senses, whereby our primary senses include vision, audition, balance, gustation, olfaction, and somatosensation. Furthermore, humans have the ability to process other conditions like e.g. temperature, or pain. There are several examples of animals evolving unique sensory mechanisms that have apparently helped them adapt to their environment, e.g. echolocation, or the perception of electric or magnetic fields. Organisms use specialized organs dedicated to each sense in order to process useful sensory information from their environment. Many species use sound detection as one of their major senses for orientation and navigation, detection of danger, and location of prey or predators. Furthermore, the generation and detection of sound is a major of communication. Because of the physical properties of a sound wave, communication through sounds is possible over long distances even under visual occlusion. In physical terms, sound is described as a periodically oscillating mechanical wave of pressure propagating through a transmission medium like air or water. Sound waves are characterized by their specific waveform, amplitude, frequency, and phase. As for any other sense, specific features of the stimulus, in this case the perceptible frequency and intensity range of sound stimuli, are confined and differ between organisms. The hearing range for humans for instance is limited to a frequency range of 20 to $20,000 \mathrm{~Hz}$ and is most sensitive between 2,000 to $5,000 \mathrm{~Hz}$. The hearing range of mice covers a frequency range from 1,000 to $100,000 \mathrm{~Hz}$ (Ashmore, 2008). Thus, they communicate using high frequency sounds, which are inaudible to humans, and do not hear lower frequencies that humans can perceive. Even though the frequency range of mice and humans differs to a large extent, mouse models enjoy great utility in the field of auditory neuroscience due to their suitability for genetic manipulation. Mouse models of hearing can help to understand the molecular mechanisms of sound encoding in the mammalian ear and serve as models for human hereditary hearing loss by unraveling the mechanisms that are disrupted, once a critical gene-mutation occurs. 


\subsection{The mammalian ear}

The mammalian ear consists of the outer, the middle, and the inner ear (Figure 1, bottom right).

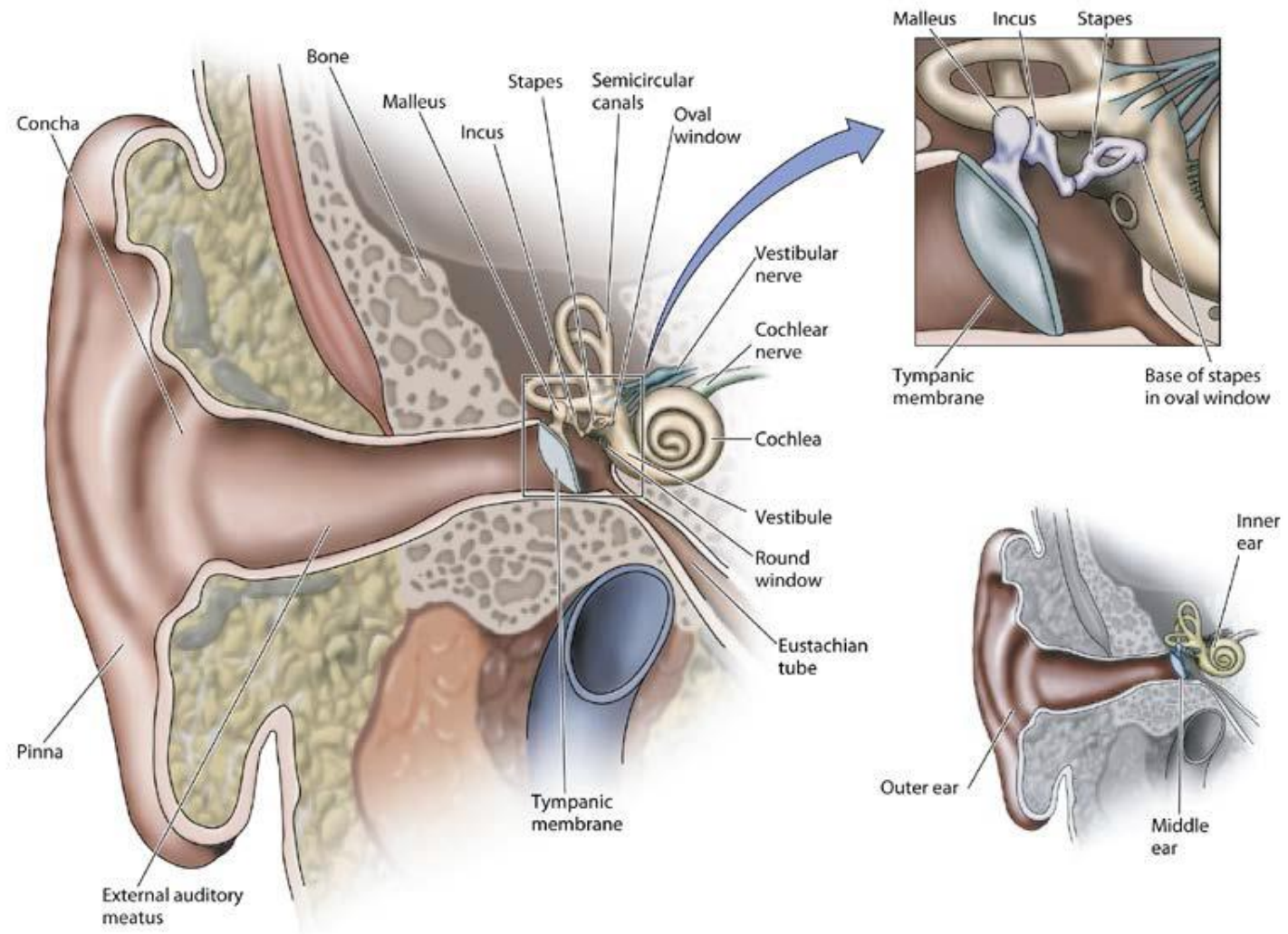

\section{Figure 1: The human ear}

The human ear (left) consists of the outer, middle, and inner ear. An overview of the main divisions of the ear is illustrated at the bottom right. The upper right panel highlights the large surface area of the tympanic membrane relative to the oval window and the three ossicles of the middle ear that form a mechanical leverage. Figure adapted from Purves et al., 2004.

\section{The outer and middle ear}

The outer ear (Figure 1) is the visible external component of the ear and consists of the auricle (pinna) and the ear canal (external acoustic meatus). The pinna functions as a sound-collector, filters and guides the gathered sound into the ear canal onto the eardrum (tympanic membrane) at the end of the canal. The incoming sound wave causes vibration of the elastic tympanic membrane, which conveys the sound to the attached ossicle chain (three ossicles: malleus, incus, and stapes) in the middle ear that couples to the fluid of the inner ear at the footplate of the stapes. The middle ear (Figure 1, top right) converts the low-impedance airborne vibrations of the eardrum into higher-impedance vibrations of the 
inner ear fluids and membranes: impedance matching. The ossicles transfer vibrations from the large, low impedance tympanic membrane to the much smaller, high impedance oval window, thereby boosting the force and reducing the displacement of the vibration suitable for driving cochlear fluids. Without this mechanical process, almost all of the acoustic energy would be reflected off the liquid high-impedance fluids of the inner ear.

\section{The inner ear and organ of Corti}

The cochlea is the acoustic part of the inner ear (Figure 2A, B) and hosts the sensory organ of Corti (Figure 2C).

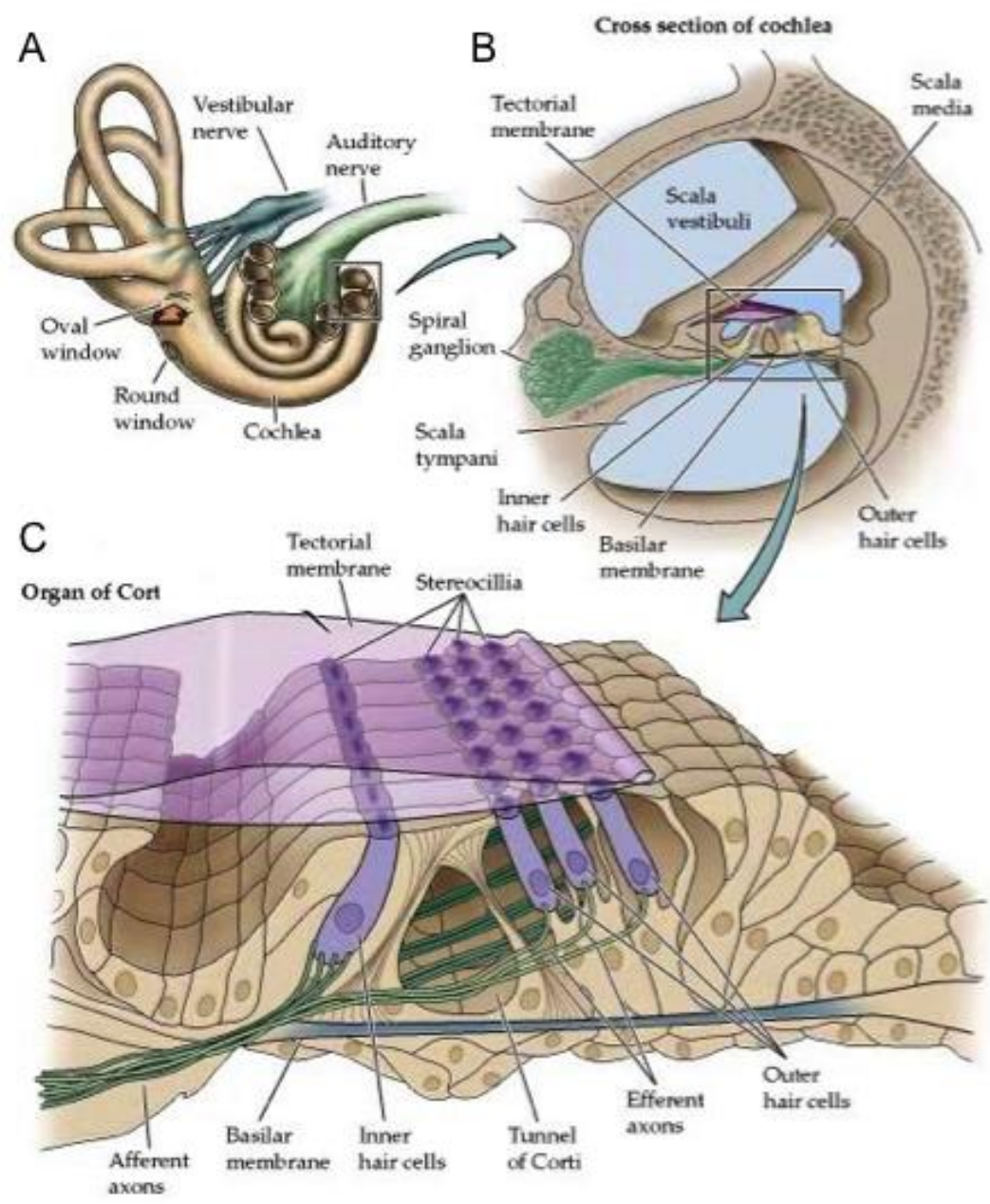

Figure 2: The human cochlea and the organ of Corti

[A] Face-on view on the cochlea showing the round and oval window at the basal end of the cochlea tube. [B] Cochlear cross-section showing the scala media between the scala vestibuli and scala tympani. [C] The organ of Corti is the hearing receptor organ, consisting of three rows of outer hair cells (OHCs) acting as cellular amplifiers, one row of IHCs, which serve as the genuine sensory cells, and several types of supporting cells. IHCs receive afferent innervation from type I SGNs, whereas OHCs receive afferent innervation from type II SGNs. Efferent input onto OHCs and type I SGNs is provided by the lateral olivocochlear neurons. Figure adapted from Purves et al., 2004 and modified. 
The cochlea is a tube-like, bony structure in the form of a coiled snail (Figure 2A) with 2.5 turns in human and 1.75 turns in mice. The cochlear partition, a flexible structure consisting of the Reissner's and the basilar membrane bisects the cochlea along its midline. It divides the cochlea into three fluid-filled compartments (Figure 2B): The scala media, the scala vestibuli above it (bordered by the Reissner's membrane), and the scala tympani below it (bordered by the basilar membrane).

Inside the cochlea, the mechanical vibration elicited by the sound wave is transformed into a neuronal code that is forwarded to higher auditory centers in the brain. The cochlea functions as frequency analyzer and thereby defines the tuning characteristics of auditory neurons.

\section{Tonotopic organization of the cochlea}

Once a sound wave arrives, the footplate of the stapes vibrates in the oval window connected to the scala vestibuli at the basal end of the cochlear tube (Figure 2A). A displacement of the cochlear fluids in the scala vestibuli causes vibration of the flexible basilar membrane eliciting a traveling wave that propagates along the cochlear duct. Due to the gradually changing resonant properties of the basilar membrane - from stiff and narrow at the base to wide and floppy at the apex - the cochlea decomposes the sound according to frequency along the duct, called tonotopy.

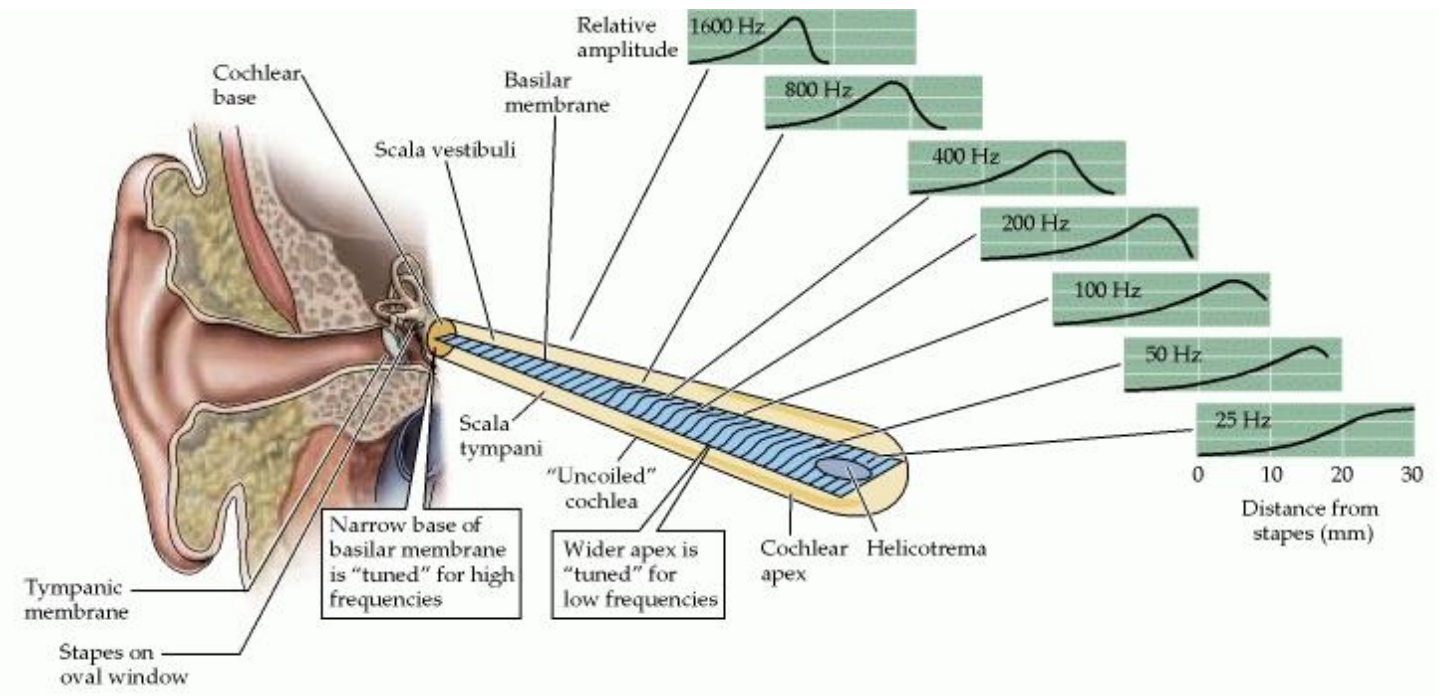

Figure 3: Tonotopic organization of the cochlea

Higher frequencies are encoded at the base and lower frequencies at the apex of the cochlea. The travelling wave moving from the base towards the apex slowly increases in amplitude and decreases in velocity. Depending on the frequency of the stimulus, the travelling wave reaches its maximal amplitude at a specific location on the basilar membrane. Figure adapted from Purves et al., 2004. 
This means that higher frequencies best vibrate the basilar membrane at the base and lower frequencies at the apex of the cochlea (Figure 3). The travelling wave always moves from the base towards the apex, slowly increasing in amplitude and decreasing in velocity. Depending on the frequency of the stimulus, the travelling wave reaches its maximal amplitude at a specific location on the basilar membrane, thereby providing frequencyselective physical separation of sound energy along the basilar membrane. This passive micromechanical decomposition of the vibrations is further sharpened by active amplification mediated by the electromotile OHCs. Each tonotopic place of the organ of Corti then transduces the mechanical signal into a neural code representing the specific sound frequency (see below).

\section{Cochlea fluids and the organ of Corti}

The scala vestibuli and scala tympani are filled with a fluid called perilymph, containing high concentrations of sodium ions $\left(\mathrm{Na}^{+}\right)$and chloride ions $\left(\mathrm{Cl}^{-}\right)$; they are connected via the helicotrema at the apex of the cochlea (Figure 3). The scala media within the cochlear partition is filled with a potassium ion $\left(\mathrm{K}^{+}\right)$rich fluid called endolymph and harbors the organ of Corti (Figure 2C).

The organ of Corti is an epithelial tissue and the hearing receptor organ of the inner ear. It is situated on top of the basilar membrane and is covered by the tectorial membrane. It consists of three rows of OHCs, which act as cellular amplifiers and one row of IHCs, the actual sensory receptors of the ear transforming vibrational energy into an electrical signal. Several types of supporting cells such as phalangeal cells, inner and outer pillar cells, Deiter's cells and Hensen's cells provide structural support and homeostasis for the organ of Corti. IHCs are innervated by afferent type I SGNs, whereas OHCs are innervated by unymelinated type II SGNs. Efferent auditory fibers arise in the brainstem and provide inhibitory input to type I SGNs beneath IHCs (lateral olivocochlear efferents) and to the OHCs (medial olivocochlear efferents), respectively.

Bulb-shaped IHCs and cylindrically-shaped OHCs were named for their bundle of hair-like stereocilia (microvilli) protruding from the cell's apex into the scala media. Stereocilia are covered by the hair cell's plasma membrane and arranged hexagonally in rows. Even though a kinocilium is missing in adult mammalian hair cells, stereocilia are arranged in a bilaterally symmetric staircase fashion. Stereocilia are connected to their next taller neighbor by tip-links - extracellular filaments containing the cell adhesion molecules protocadherin 15 and cadherin 23 (Müller, 2008; Pickles et al., 1984; Siemens et al., 2004; Söllner et al., 2004). Tip-links are directly linked to mechano-electrical transducer (MET) 
channels (Assad et al., 1991; Howard and Hudspeth, 1988) - specialized cation selective mechanoreceptors with ultrafast activation kinetics and large single-channel conductance (Beurg et al., 2006; Corey and Hudspeth, 1983; Fettiplace, 2016; Fettiplace and Kim, 2014; Ricci et al., 2005). The tallest stereocilia of OHCs are thought to directly connect to the overlaying tectorial membrane. Movements of the basilar and tectorial membranes relative to each other creates a shearing motion, causing horizontal displacement of the stereociliar bundle. An actin cytoskeleton provides structural stability to the stereocilia, allowing bidirectional deflection, which causes either depolarization or hyperpolarization of hair cells. Parallel movement of stereocilia towards the tallest stereocilium in the center creates tension in the tip links and thus induces depolarizing $\mathrm{K}^{+}$influx from endolymph in the scala media into the hair cells through opening of the MET channels. The ionic flow into the hair cells results in a graded receptor potential following the stereocilia's movement. This way the temporal information from the sinusoidal sound stimulus can be preserved.

The driving force for depolarizing $\mathrm{K}^{+}$influx into the hair cells is based on an electrical gradient across the hair cell membrane and the endolymph in the scala media. The resting potential of hair cells in the organ of Corti is about $-45 \mathrm{mV}$. The high $\left[\mathrm{K}^{+}\right]$and low $\left[\mathrm{Na}^{+}\right]$ concentration in the scala media (Nernst potential for $\mathrm{K}^{+}$over the apical hair cell membrane: $0 \mathrm{mV}$ ) and the positive potential of approximately $+80 \mathrm{mV}$ in the endolymph with respect to the perilymph-filled compartments (endocochlear potential) result in a strong electrical gradient for $\mathrm{K}^{+}$(about $125 \mathrm{mV}$ ). This fuels the mechanoelectrical transduction process, with little energetic burden for hair cells that can, at the end of depolarization, simply extrude the $\mathrm{K}^{+}$into the $\mathrm{K}^{+}$-poor perilymph along the typical electrochemical gradient through voltagegated $\mathrm{K}^{+}$-channels at the basolateral membrane. The high $\left[\mathrm{K}^{+}\right]$-concentration in the scala media is maintained through active ion transport by cells of the multilayered epithelium of the stria vascularis, lining the cochlear duct in the scala media.

In OHCs, this $\mathrm{K}^{+}$-driven depolarization causes voltage-dependent conformational changes of the integral membrane protein prestin (Zheng et al., 2000), which shrinks during depolarization, resulting in changes of the OHC's length at the same frequency as the incoming sound wave. Thereby, OHCs enhance vibrations of the tectorial membrane at particular locations along the cochlea by their electro-motility. These active movements of OHCs further sharpen the frequency resolving power of the cochlea by providing soundevoked mechanical feedback amplification (Ashmore, 2008; Ashmore et al., 2010; Hudspeth, 2008; Reichenbach and Hudspeth, 2010). In IHCs, the $\mathrm{K}^{+}$-driven depolarization induces opening of voltage-gated calcium $\left(\mathrm{Ca}^{2+}\right)$ channels at synaptic $\mathrm{AZs}$ at the basolateral membrane. Synaptic $\mathrm{Ca}^{2+}$-influx triggers the exocytosis of the neurotransmitter glutamate 
into the synaptic cleft. Glutamate is sensed by a-amino-3-hydroxy-5-methyl-4isoxazolepropionic acid (AMPA) receptors at the postsynaptic terminal of the afferent SGNs, which eventually fire and propagate their action potentials (APs) towards the auditory brainstem.

\subsection{Inner hair cell ribbon synapses}

Ribbon synapses were named after an electron dense organelle - the ribbon - tethering a halo of SVs. It is present at AZs of auditory and vestibular hair cells, photoreceptors and bipolar cells in the retina, and in pinealocytes of the pineal gland (reviewed in: Fuchs, Glowatzki, and Moser 2003; Lenzi and Von Gersdorff 2001; Sterling and Matthews 2005). By contrast to conventional synapses, where the fundamental features of neurotransmitter release are highly conserved and governed by presynaptic APs, ribbon synapses are specialized for graded and sustained neurotransmitter release: ribbon synapses release neurotransmitter in response to graded membrane potentials. These somatic potentials can be as small as a few $\mathrm{mV}$ or less and are modulated with respect to the stimulus intensity (Von Gersdorff, 2001). This feature allows IHCs to encode sounds of very different intensities with high sensitivity covering a large dynamic range. Further, patch-clamp recordings of presynaptic IHC membrane capacitance (Khimich et al., 2005; Moser and Beutner, 2000; Parsons et al., 1994; Rutherford and Roberts, 2006) and of postsynaptic excitatory currents from afferent nerve terminals (Glowatzki and Fuchs, 2002; Keen and Hudspeth, 2006; Li et al., 2009) revealed that IHC ribbon synapses employ precise signaling at sustained and high rates of transmitter release (hundreds of vesicles/s) to indefatigably track the ever-present stimulating sounds (reviewed in: Moser and Vogl 2016; Reijntjes and Pyott 2016; Safieddine, El-Amraoui, and Petit 2012; Wichmann and Moser 2015).

\section{The synaptic ribbon and vesicle pools}

The ribbon is a hallmark feature of ribbon synapses. To date the ribbon function has been investigated by a variety of morphological and physiological studies. However, despite major investigations its functional role remains debated. The synaptic ribbon is a regularly arranged dense projection mainly consisting of the structural component RIBEYE (Khimich et al., 2005; Maxeiner et al., 2016; Schmitz et al., 2000). Genetic RIBEYE disruption in mice abolished all presynaptic ribbons in retinal ribbon synapses, impaired fast and sustained neurotransmitter release, and changed $\mathrm{Ca}^{2+}$ nanodomain-like coupling of exocytosis in rod bipolar cells (Maxeiner et al., 2016). Regularly arranged dense projections such as the 
ribbon can be also observed at the frog or Drosophila melanogaster neuromuscular junction (NMJ) (Harlow et al., 2001; Szule et al., 2012; Wichmann and Sigrist, 2010). In Drosophila melanogaster, however, the main structural component of the dense projection called the T-bar is not RIBEYE, but bruchpilot (Kittel, 2006; Wagh et al., 2006) - the invertebrate homolog of CAST/ERC2 (ELKS2) (Ohtsuka et al., 2002; Wagh et al., 2006).

Based on electron microscopy and electron tomography studies of IHC ribbon synapses, several distinct morphological populations of SVs have been described (Khimich et al., 2005; Lenzi et al., 1999, 2002; Schnee et al., 2005). Besides free cytosolic SVs, pools of membrane-proximal (MP) as well as ribbon-associated (RA) SVs can be distinguished (Jung et al., 2015a). Often, SVs are structurally attached to the ribbon and/or the plasma membrane through filamentous tethers (Jung et al., 2015a; Lenzi et al., 1999; Nouvian et al., 2006; Wichmann and Moser, 2015). In this work, the following nomenclature for SV pools is used (illustrated in Figure 19A): The RA-SV pool comprises a SV monolayer around the synaptic ribbon within a distance of $80 \mathrm{~nm}$ from the ribbon (Jung et al., 2015a). The pool of MP-SVs is comprised of SVs at the ribbon base, which are in close proximity $(\leq 50 \mathrm{~nm})$ to the plasma membrane and within a distance of $100 \mathrm{~nm}$ to the presynaptic density (PD) (Jung et al., 2015a). Another MP SV population can be found more distal from the ribbon, termed "outlying"-SV pool (Lenzi et al., 2002). In this work, "outlying"-SVs are defined as SVs that are located within $50 \mathrm{~nm}$ to the presynaptic plasma membrane, more than $100 \mathrm{~nm}$ away from the PD, but directly opposed to the postsynaptic density.

Physiologically, exocytic SV fusion with the IHC's plasma membrane can be monitored as changes in membrane capacitance $\left(\Delta \mathrm{C}_{\mathrm{m}}\right)$ using the patch-clamp technique (reviewed in Neher 1998). SV fusion with the plasma membrane leads to an increase in membrane surface and thus in membrane capacitance. In IHCs, different kinetic components of exocytosis can be observed according to the changes in membrane capacitance with respect to step depolarizations of varying length. There is a fast and saturating kinetic component that can be estimated by the exocytic $\Delta \mathrm{C}_{\mathrm{m}}$ response of $\mathrm{IHCs}$ during depolarizations of up to $20 \mathrm{~ms}$. This component likely reflects a small, finite pool of SVs that is depleted rapidly. Thus, it was referred to as the readily releasable pool (RRP) (Moser and Beutner, 2000; Rutherford and Roberts, 2006; Spassova et al., 2004). Further, there is a slower, linear kinetic component of exocytosis ( $\geq 50 \mathrm{~ms}$ depolarization). It was attributed to the sustained phase of SV release, limited by SV resupply after RRP depletion (Frank et al., 2010; Goutman and Glowatzki, 2007; Meyer et al., 2009; Schnee et al., 2005). 
Relating morphological to physiological findings may help to understand SV pool dynamics and the functional role of the synaptic ribbon in more detail. This is however complicated by technical issues. Due to variations in morphological SV counts as well as in approximations of single SV capacitance it has not been yet possible to arrive at a common anatomical and physiological definition for the pool of SVs undergoing release during the linear sustained phase of exocytosis. This phase of release might include serial resupply of SVs or release of SVs from the MP "outlying" SV pool more distal from the ribbon (Lenzi et al., 1999, 2002; Nouvian et al., 2006). Nonetheless, correlations between the physiologically described RRP and morphological studies revealed mechanistic insights into SV release at IHC ribbon synapses. Recent work suggests that the physiologically measured RRP employs the fusion of docked SVs from the morphologically defined MP-SV pool (Khimich et al., 2005; Moser and Beutner, 2000; Nouvian et al., 2006; Rutherford and Roberts, 2006; Schnee et al., 2005). A second hypothesis states that the fast kinetic component of exocytosis additionally involves the exocytosis of RA-SVs. This requires either fast recruitment of RA-SVs to the plasma membrane or compound (pre-fused SVs) and cumulative (serial homotypic) SV fusion with the membrane, leading to multivesicular release (Edmonds et al., 2004; Spassova et al., 2004). Different experimental approaches suggest several functional roles for the ribbon on SV dynamics. The synaptic ribbon may act as (1) a scaffold organizing the $A Z$ and promoting the large and spatially confined array of $\mathrm{Ca}^{2+}$-channels and membrane proximal vesicles (Frank et al., 2010; Khimich et al., 2005), which in the retina seems to be critical also for the tight coupling (Maxeiner et al., 2016) (2) a „conveyor belt“ - allowing fast SV transport to the plasma membrane by passive diffusion of SVs through transient interactions of ribbon bound tethers with the SVs (Graydon et al., 2014) or active shuttling of ribbon attached SVs to the plasma membrane (Bunt, 1971; Von Gersdorff, 2001; Vaithianathan et al., 2016) (3) a "safety belt“ - decreasing the speed of SV diffusion, enabling compound fusion of SVs (Matthews and Sterling, 2008; Parsons and Sterling, 2003). The notion of multivesicular release was founded on the observation of large variations in the amplitude of monophasic EPSCs (excitatory postsynaptic current) as well as the presence of multiphasic EPSCs in postsynaptic patch-clamp recordings from afferent SGN terminals (Glowatzki and Fuchs, 2002). While the amplitude variation of fast monophasic EPSCs was proposed to reflect highly synchronized multivesicular release, the multiphasic EPSCs where thought to reflect the release of multiple quanta in an unsynchronized way (Glowatzki and Fuchs, 2002). Alternatively, a model of univesicular release with dynamic fusion pore regulation was proposed, in which multiphasic EPSCs were interpreted as step-by-step neurotransmitter release through a flickering fusion pore (Chapochnikov et al., 2014). 


\section{Molecular key players}

A network of presynaptic proteins constitutes the cytomatrix of the $A Z(C A Z)$, orchestrating the highly specialized sound-stimulus coding at the IHC-SGN synapse through tight stimulus-secretion coupling mediated by voltage-gated $\mathrm{Ca}^{2+}$-channels.

\section{Voltage-gated $\mathrm{Ca}^{2+}$-channels}

Voltage-gated $\mathrm{Ca}^{2+}$-channels are key players in $\mathrm{IHC}$ neurotransmitter release. Exocytosis is triggered by influx of $\mathrm{Ca}^{2+}$ through mainly ( 90\%) L-type voltage-gated Cav1.3 $\mathrm{Ca}^{2+}$ channels that are clustered at the PD underneath the ribbon (Baig et al., 2011; BechHansen et al., 1998; Brandt et al., 2003; Dou et al., 2004; Mansergh et al., 2005; Platzer et al., 2000; Strom et al., 1998). Cav1.3 $\mathrm{Ca}^{2+}$-channels activate at relatively hyperpolarized potentials, which makes them suitable to react to subtle graded potential changes, and inactivate slowly, providing tonic $\mathrm{Ca}^{2+}$-influx and neurotransmitter release (Baumann et al., 2004; Koschak et al., 2001, 2003; Xu and Lipscombe, 2001; reviewed in Joiner and Lee, 2015). Each $A Z$ of mature mouse IHCs from the apical cochlear turn is assumed to cluster on average approximately $\sim 80-100 \mathrm{Ca}^{2+}$-channels (Brandt et al., 2005; Wong et al., 2014). It was suggested that Cav1.3 $\mathrm{Ca}^{2+}$-channels are tightly coupled to SVs of the RRP with an effective weighted coupling distance of only 15-17 nm (Pangršič et al., 2015). In this socalled nanodomain-like control of exocytosis the release of SVs is linearly dependent on the number of open $\mathrm{Ca}^{2+}$-channels (Figure 4, left). Experimentally, RRP exocytosis in mature apical mouse and gerbil IHCs from the low-frequency apical cochlear region showed a low sensitivity to intracellular application of the slow-binding $\mathrm{Ca}^{2+}$-buffer EGTA further supporting the hypothesis of a tight coupling between $\mathrm{Ca}^{2+}$-channels and the SVs (Brandt et al., 2005; Johnson et al., 2017). Physiologically, nanodomain-like control of SV exocytosis might be especially suitable and important for graded sensory signals of IHCs to (1) encode sound stimuli over a large dynamic range starting from very weak sounds, because a tight coupling provides high $\mathrm{Ca}^{2+}$-sensitivity, (2) provide high temporal precision of exocytosis and phase-locking, because tight coupling increases the speed and efficacy of exocytosis and reduces synaptic delays (Moser et al., 2006a) and (3) work energyefficient, because less and locally confined intracellular $\mathrm{Ca}^{2+}$ needs to be cleared (Bartoletti et al., 2011; Brandt et al., 2005; Jarsky et al., 2010; Thoreson et al., 2004). 
$\mathrm{Ca}^{2+}$ nanodomain-like coupling

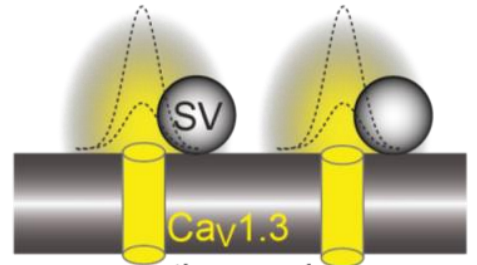

presynaptic membrane
$\mathrm{Ca}^{2+}$ microdomain-like coupling

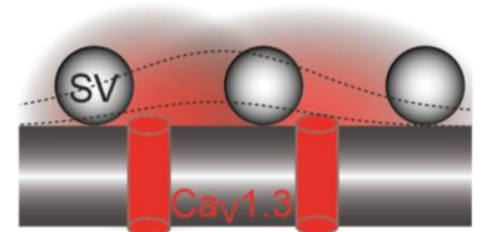

presynaptic membrane

Figure 4: Coupling between voltage-gated $\mathrm{Ca}^{2+}$-channels and SVs

[Left] Tight nanodomain-like coupling of SVs and voltage-gated $\mathrm{Ca}^{2+}$-channels (yellow) leads to locally confined changes in intracellular $\left[\mathrm{Ca}^{2+}\right]_{i}$ (dashed lines) and thereby precise control of exocytosis. In such a scenario, the release of SVs is linearly dependent on the number of open $\mathrm{Ca}^{2+}$-channels. [Right] Looser $\mathrm{Ca}^{2+}$ microdomain-like coupling employs opening of several $\mathrm{Ca}^{2+}$-channels (red) with overlapping domains (dashed lines) for the fusion of a single SV and a large $\mathrm{Ca}^{2+}$-channel to SV distance of more than $100 \mathrm{~nm}$. Hence, the release of SVs is non-linearly dependent on the number of open $\mathrm{Ca}^{2+}$-channels. Image not to scale.

Some synapses of the central nervous system however use a micodomain-like control of exocytosis (Figure 4, right): This employs opening of several $\mathrm{Ca}^{2+}$-channels with overlapping domains for the fusion of a single SV and a large $\mathrm{Ca}^{2+}$-channel to SV distance of more than $100 \mathrm{~nm}$ (Borst and Sakmann, 1996; Eggermann et al., 2011). In such a scenario, the release of SVs is non-linearly dependent on the number of open $\mathrm{Ca}^{2+}$ channels. Physiologically, microdomain-like control of SV exocytosis has a positive impact on (1) the signal-to-noise ratio, since the high $\mathrm{Ca}^{2+}$ cooperativity (4-5) of SVs reduces SV fusion below a certain threshold and the noise resulting from single channel gating is efficiently averaged out as well as (2) the synchronization of SV fusion, since the $\mathrm{Ca}^{2+}$ domain overlap triggers simultaneous fusion of several SVs upon reaching a certain threshold (Matveev et al., 2011; Schneggenburger and Neher, 2005). Looser microdomainlike $\mathrm{Ca}^{2+}$ - SV coupling was observed in gerbil IHCs towards the high-frequency tuned cochlear base (Johnson et al., 2017). Compared to the generally low frequency tuned cochlea of the gerbil $(0.3-30 \mathrm{kHz})$, the high-frequency tuned cochlea of mice $(4-100 \mathrm{kHz})$ might however have different properties.

\section{CAZ proteins}

Morphological studies of central nervous system synapses have shown that the CAZ forms a presynaptic grid of electron dense material at the synaptic $A Z$, extending approximately $50 \mathrm{~nm}$ into the cytoplasm (Fenster et al., 2000; Limbach et al., 2011; Phillips et al., 2001; Südhof, 2012; Zhai and Bellen, 2004). A filamentous network protruding further into the 
cytoplasm is associated with SVs and regulates SV dynamics and docking (Cole et al., 2016; Zhai and Bellen, 2004). In neuronal synapses, bassoon, piccolo, liprins- $\alpha$, UNC13/Munc13s, CASTs/ERCs (ELKS), RIMs (Rab3-interacting molecules) and RIM-BPs (RIM-binding proteins) form the core of the presynaptic cytomatrix and are essential for the organization of docking/priming, release and retrieval of SVs, and localization of voltagegated $\mathrm{Ca}^{2+}$-channels to release sites for efficient stimulus-secretion coupling. CAZ proteins interact with each other and are associated with SV fusion proteins, cytoskeletal and scaffolding proteins, cell adhesion molecules, and voltage-gated $\mathrm{Ca}^{2+}$-channels for maintaining the structural and functional organization of the AZ (Gundelfinger and Fejtova, 2012; Schoch and Gundelfinger, 2006). Recent work revealed that IHC ribbon synapses work independently of the CAPS and Munc-13 1/2 families of priming proteins (Vogl et al., 2015) and neuronal SNAREs (Nouvian et al., 2011). So far, only few CAZ proteins have been found to be present in IHC ribbon synapses and are functionally characterized.
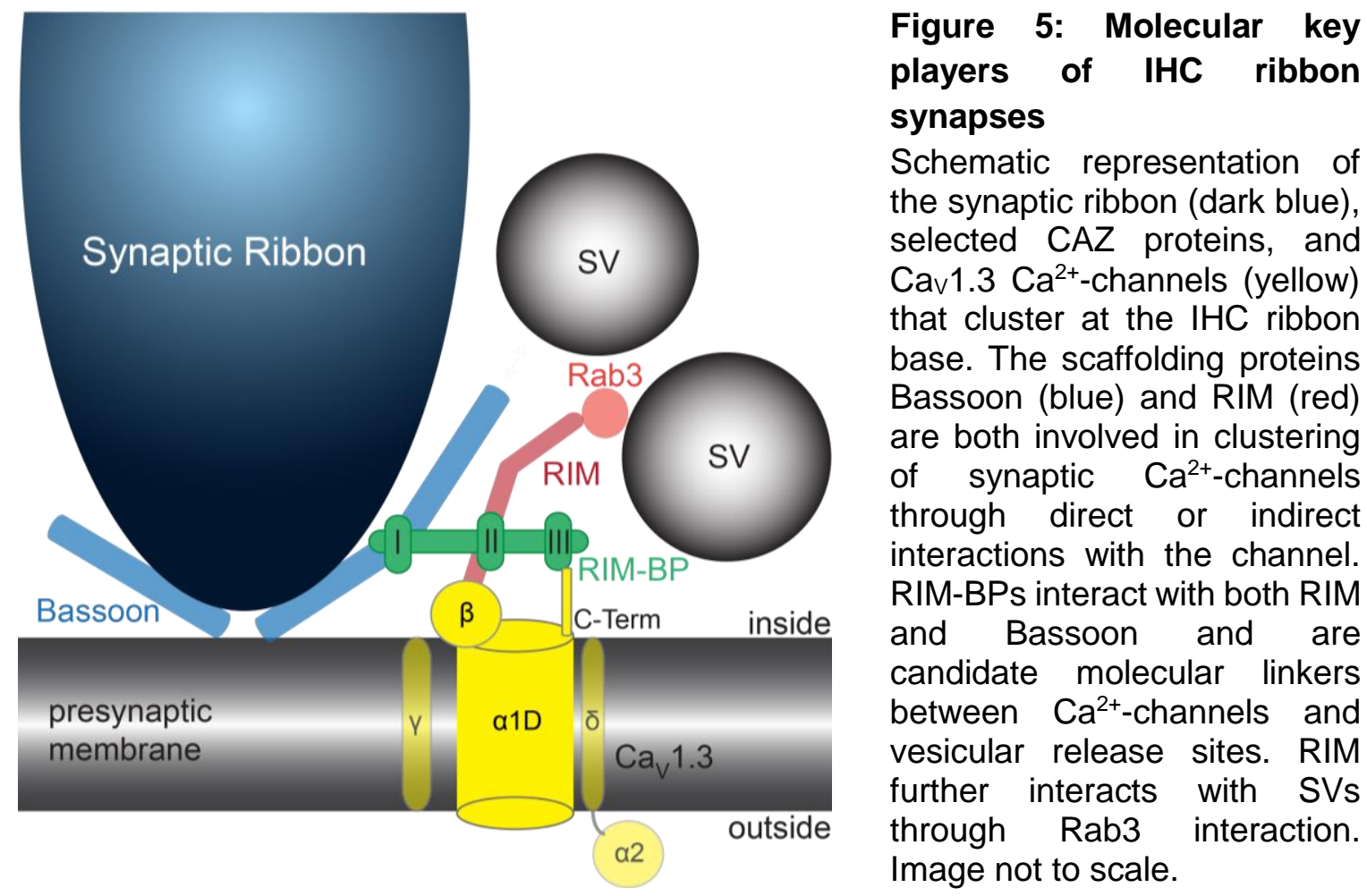
Schematic representation of the synaptic ribbon (dark blue), selected CAZ proteins, and Cav1.3 $\mathrm{Ca}^{2+}$-channels (yellow) that cluster at the $\mathrm{IHC}$ ribbon base. The scaffolding proteins Bassoon (blue) and RIM (red) are both involved in clustering of synaptic $\mathrm{Ca}^{2+}$-channels through direct or indirect interactions with the channel. RIM-BPs interact with both RIM and Bassoon and are candidate molecular linkers between $\mathrm{Ca}^{2+}$-channels and vesicular release sites. RIM further interacts with SVs through Rab3 interaction. Image not to scale.

The large scaffold protein bassoon (Figure 5, blue) is responsible for the structural and functional integrity of the IHC AZ. Genetic disruption of bassoon in mice revealed that bassoon anchors the synaptic ribbon to the plasma membrane (Dick et al., 2003; Khimich et al., 2005; Tom Dieck et al., 2005), is further involved in SV-replenishment, and enhances synchronous auditory signaling by promoting $\mathrm{Ca}^{2+}$-channel clustering, thereby creating SV release sites at IHC AZs (Frank et al., 2010; Khimich et al., 2005). Bassoon however does 
not directly interact with voltage-gated $\mathrm{Ca}^{2+}$-channels, but controls presynaptic P- and Qtype $\mathrm{Ca}^{2+}$-channels via RIM-BP interaction (Davydova et al., 2014). Besides bassoon, the multidomain scaffolding protein family of RIM proteins (Figure 5 , red) yields another group of molecular key players at IHC AZs (Jung et al., 2015a; Picher et al., 2017). RIM1 is absent in IHCs, but two isoforms of RIM2 (RIM2 $\alpha$ and RIM2 $\beta$ ) were found to be involved in the tethering of SVs to the presynaptic $A Z$ as well as in the regulation of presynaptic $\mathrm{Ca}^{2+}$ channel abundance and clustering, thereby creating SV release sites for RRP exocytosis (Jung et al., 2015a). RIMs tether voltage-gated $\mathrm{Ca}^{2+}$-channels to the AZ either directly or indirectly (Coppola et al., 2001; Gebhart et al., 2010; Grabner et al., 2015; Han et al., 2011; Jung et al., 2015a; Kaeser et al., 2011; Kintscher et al., 2013; Kiyonaka et al., 2007; Picher et al., 2017). The deletion of RIM2 $\alpha$ and bassoon, however, did neither lead to a complete loss of synaptic $\mathrm{Ca}^{2+}$-channels, nor perturb the coupling between $\mathrm{Ca}^{2+}$-channels and $\mathrm{SVs}$ (Frank et al., 2010; Jung et al., 2015a). This suggests that additional molecular linkers may help clustering Cav1.3 $\mathrm{Ca}^{2+}$-channels at the IHC AZ and localize them in close proximity to SVs to provide molecular coupling between SVs and the priming-fusion apparatus at the release site.

In conventional synapses and Drosophila melanogaster NMJs, RIM-BPs were found to play such a role, acting as molecular junction between voltage-gated $\mathrm{Ca}^{2+}$-channels and SVs (Acuna et al. 2015; Grauel et al. 2016; Hibino et al. 2002; Kaeser et al. 2011; M. Müller, Genç, and Davis 2015). Studies from the calyx of Held reported an increased sensitivity of SV release to the slow $\mathrm{Ca}^{2+}$-buffer EGTA, proposing an increased coupling distance between $\mathrm{Ca}^{2+}$-channels and SVs in absence of RIM-BP1 and -2 (Acuna et al. 2015). In hippocampal synapses, RIM-BP2 was found to fine-tune the clustering of P/Q-type $\mathrm{Ca}^{2+}$ channels at the $\mathrm{AZs}$, which affected the $\mathrm{Ca}^{2+}$-nanodomains and reduced the initial release probability of SVs (Grauel et al., 2016). In Drosophila melanogaster NMJs, the RIM-BP ortholog DRBP was found to be essential for both the structural and functional integrity of the $\mathrm{AZ}$, reporting impaired $\mathrm{Ca}^{2+}$-channel clustering and $\mathrm{Ca}^{2+}$-influx as well as looser $\mathrm{Ca}^{2+}$ influx-exocytosis coupling upon DRBP disruption (Liu et al., 2011; Müller et al., 2015). The expression and function of RIM-BPs have not been studied in IHCs so far. Nevertheless, given their role in other synapses, they are promising candidates to regulate the abundance of presynaptic $\mathrm{Ca}^{2+}$-channels and to contribute to the $\mathrm{Ca}^{2+}$ nanodomain-like control of $\mathrm{SV}$ exocytosis at the IHC AZ. 


\section{$2.3 \quad$ Spiral ganglion neurons}

\section{Cochlear innervation pattern and spike generation}

SGNs innervate the sensory hair cells and thereby connect the peripheral sensory receptor system with the brain. SGNs are bipolar neurons and their somata are located in the spiral ganglion in the Rosenthal's canal surrounded by the modiolus, the bony central axis of the cochlea. Their central processes (ANFs) form the cohlear part of the VIIlth cranial nerve, which projects to the neurons of the cochlear nucleus in the auditory brainstem. Depending on their pattern of peripheral innervation, SGNs are grouped into two classes: Type I SGNs contain the vast majority (90-95\%) of SGNs and project onto the IHCs (Figure 6B). Each type I SGN receives input from a single IHC ribbon-type AZ (one-to-one). Type I SGNs are myelinated and give rise to inner radial fibers of the cochlea that project to the brainstem. Each SGN fires preferentially to a specific frequency, determined by the position of the contacted IHC along the tonotopic map of the cochlea. Type II SGNs represent the remaining $5-10 \%$ of SGNs that are unymelinated, highly branched, and contact multiple OHCs en passant or terminally (Figure 6B).

In the organ of Corti, all axons are unmyelinated. They exit the organ of Corti through the foramina nervosa (asterisk in Figure $6 \mathrm{~A}$ and $\mathrm{B}$ ) through the basilar membrane, before the myelinated axonal segments begin (Figure $6 \mathrm{~A}$ and $\mathrm{B}$ ). Upon glutamate release from IHC ribbon synapses, boutons of type I SGNs generate EPSCs through AMPA receptors that are clustered at postsynaptic boutons of type I SGNs (Figure $6 \mathrm{~A}$ ) juxtaposed to each IHC ribbon-type AZ leading to excitatory post-synaptic potentials (EPSPs) (Glowatzki and Fuchs, 2002; Grant et al., 2010; Meyer et al., 2009). APs are generated in the spike generator, which is presumably located in the first heminode central to the foramina nervosa (Hossain et al., 2005). High densities of Nav1.6 channels are situated at the unmyelinated type I SGN segment and at the nodes of Ranvier between the myelinated axon segments (Figure 6B, red) to mediate rapid AP generation and propagation (Hossain et al., 2005; Lacas-Gervais et al., 2004; Rutherford et al., 2012). In vitro patch-clamp experiments demonstrated that almost every EPSP, and thus every vesicle released from an IHC, elicits an AP (Rutherford et al., 2012). SGN spiking patterns encode important stimulus parameters such as intensity and timing of the sound (Meyer and Moser, 2010). Together, the large amplitudes of EPSCs (on average -148 pA) and the high densities of Nav1.6 channels in the spike generator enable SGNs to accurately encode temporal sound information (Glowatzki and Fuchs, 2002; Rutherford et al., 2012). 


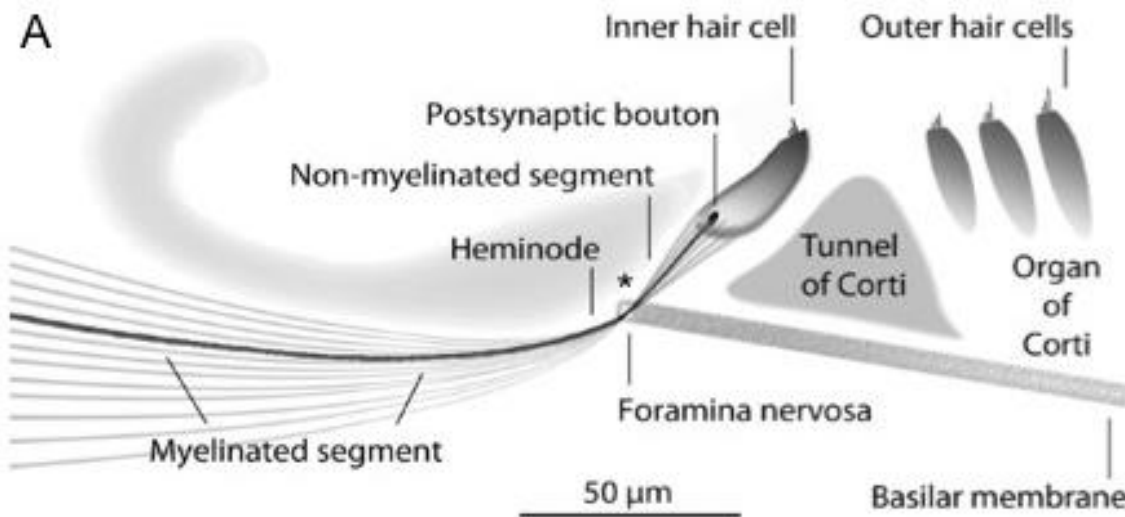

B

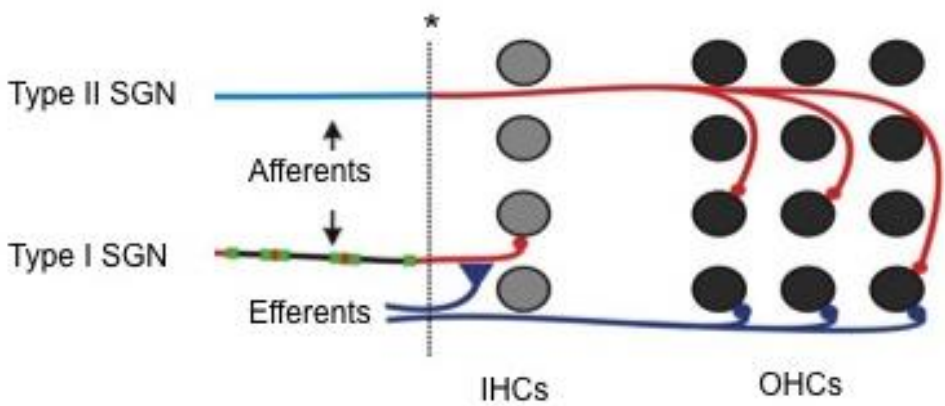

Figure 6: Scheme of cochlear innervation

Schematic cross section [A] and top-view [B] of the organ of Corti: Afferent fibers from type I SGN innervate individual IHCs (B: gray) in a one-to-one fashion. Multiple OHCs (B: black) are innervated by afferent type II SGNs en passant or terminally. Efferent ANFs (B: dark blue) provide inhibitory input to type I SGNs beneath the IHCs. All fibers exit the organ of Corti through the foramina nervosa (asterisk) through the basilar membrane, where the myelinated axonal segments start. APs of type I SGNs are triggered in the spike generator, which is presumably located in the first heminode central to the foramina nervosa and contains a high density of Nav1.6 channels (B: red). Images adapted from Hossain et al. 2005, and Rutherford, Chapochnikov, and Moser 2012 and modified.

\section{Heterogeneous response characteristics}

SGN responses to sound stimuli in vivo are studied using extracellular recordings from ANFs, which revealed great variability in spike response characteristics to the same sound stimulus. The most prominent property of SGNs is their frequency selectivity. Each SGN preferentially elicits high discharge rates at a specific acoustic frequency where it shows maximal sensitivity - the characteristic frequency. This is largely determined by the innervation location on the cochlear tonotopic axis and enhanced by $\mathrm{OHC}$ amplification (Kiang et al., 1965; Liberman, 1978; Taberner and Liberman, 2005). However, even SGNs with very similar characteristic frequency differ: e.g. in their spontaneous spike rate (ranges from 0-120 Hz), spike threshold, and dynamic spike range (Liberman, 1978; Ohlemiller and Echteler, 1990; Sachs and Abbas, 1974; Taberner and Liberman, 2005; Yates et al., 1990). The spontaneous spike rate is defined as the spike rate in the absence of sound stimuli, due to spontaneous SV release by the ribbon synapse. The spike threshold of an SGN is 
defined as the stimulus strength ( $\mathrm{dB} S \mathrm{SP}$ ) that is required to evoke a change in spike rate of the SGN at their characteristic frequency. Both, the spontaneous spike rate and spike threshold vary greatly among SGNs and are inversely related to each other (Liberman, 1978; Taberner and Liberman, 2005; Yates et al., 1990). The range of sound intensities over which changes in the evoked spike rates can be measured, is called the dynamic range of a SGN. It positively correlates with the spike threshold and negatively correlates with the spontaneous spike rate (Yates et al., 1990). Thus, SGNs with a high spontaneous spike rate (high-SR SGNs) usually have a low spike threshold and narrow dynamic range (Figure 7 , red box).

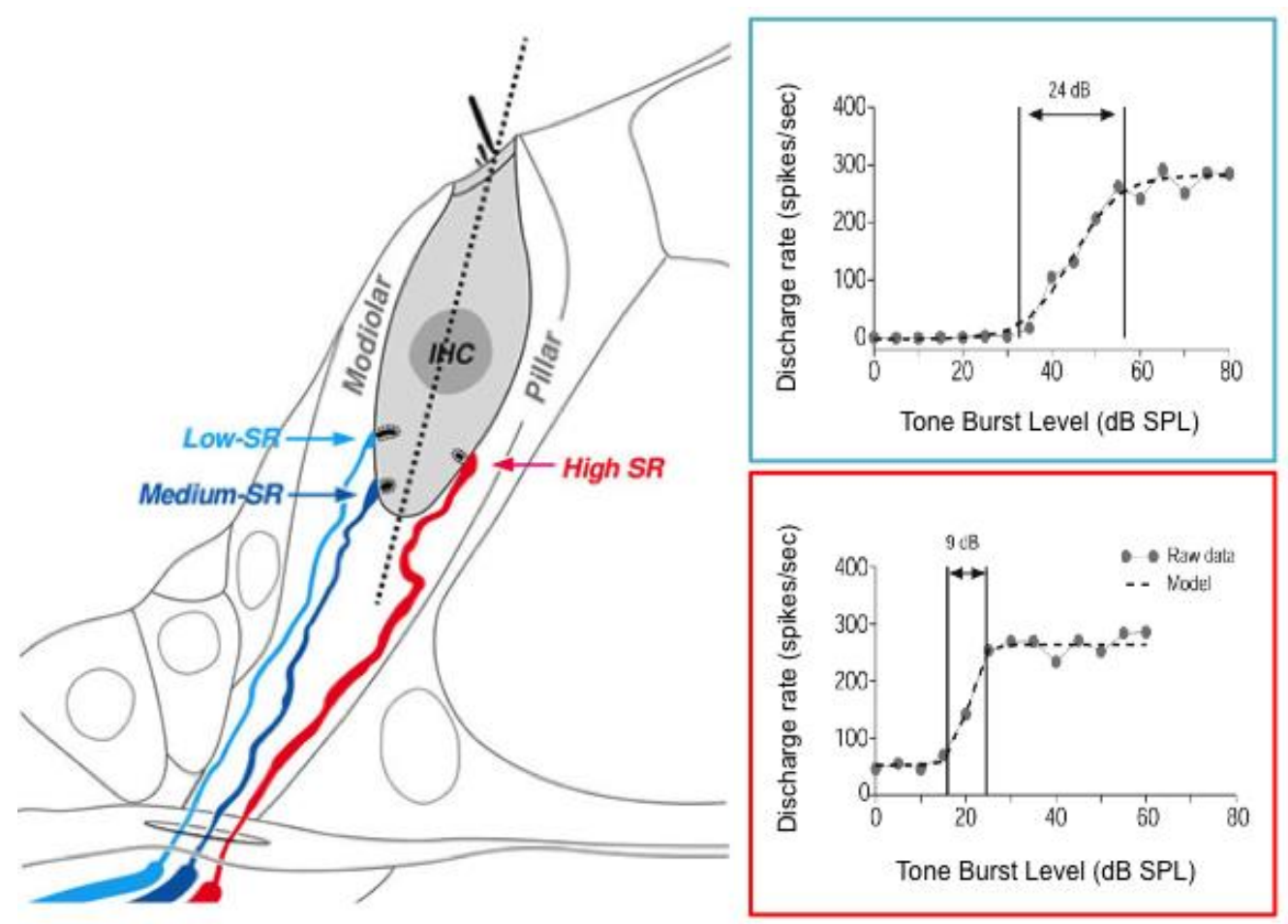

Figure 7: Heterogeneous response characteristics of type I SGNs innervating ribbon synapses of IHCs

Right: SGNs are diverse regarding their spontaneous spike rate, spike threshold and dynamic spike range (black lines). Left: A differential innervation pattern for low/mediumSR (blue fiber) and high-SR (red fiber) SGNs regarding the IHC symmetry axis has been described in cat (Kawase and Liberman, 1992; Liberman, 1980, 1982; Liberman and Oliver, 1984). Characteristically, high-SR SGNs have a low spike threshold, narrow dynamic range, and innervate the IHC on the pillar side (facing OHCs) (red fiber, red box), whereas lowSR SGNs have a high spike threshold, broad dynamic range and innervate the $\mathrm{IHC}$ on the modiolar side (facing the spiral ganglion) (blue fiber, blue box). Image adapted from Bharadwaj et al. 2014 and Taberner and Liberman 2005 and modified.

Hence, high-SR SGNs change their firing in response to soft sounds and saturate quickly when the sound intensity rises. Then, low spontaneous spike rate SGNs (low-SR SGNs), having a high spike threshold and broader dynamic spike range, are recruited (Figure 7, blue box). Since the dynamic range of individual SGNs is small $(<43 \mathrm{~dB})$ compared to the 
range of audible sound intensities ( 120 dB) (Taberner and Liberman, 2005), it is believed that SGNs complement each other through their functional diversity in their response characteristics. Thereby, they enable the organism to encode sounds over the full dynamic range of audible sound intensities with high sensitivity.

Several pre- and postsynaptic mechanisms may contribute to the observed functional diversity of SGNs. Differences in the afferent synapse molecular anatomy (Merchan-Perez and Liberman, 1996), as well as efferent modulation of SGNs (Ruel et al., 2001) have been proposed so far. (1) Functionally, heterogeneous SGN response characteristics could be caused by a high variability in EPSC shape and amplitude, which might be due to variations in the number and density of postsynaptic AMPA receptors (Chen et al., 2007; Grant et al., 2010; Liberman et al., 2011; Rutherford et al., 2012). Rapid adaptation and modulation of SGN responses could further be shaped by efferent fibers that primarily innervate low-SR SGNs (Groff and Liberman, 2003; Le Prell et al., 2005; Ruel et al., 2001). (2) Anatomically, it was found that afferent fibers vary in their diameter. In cat, low/medium-SR SGNs have a smaller diameter compared to high-SR SGNs (Liberman 1982). The large diameter of highSR SGNs may facilitate AP initiation and propagation and thus explain the lower spike thresholds of these fibers and vice versa (Geisler et al., 1985). Further, a positive correlation between the innervation pattern of SGNs to the IHC and their response characteristics has been described in cat. Low-SR SGNs preferentially appear to innervate the modiolar side (facing the spiral ganglion) of the IHC, whereas high-SR SGNs preferentially innervate the opposite pillar side of the IHC (facing the OHCs) (Figure 7, left) (Kawase and Liberman, 1992; Liberman, 1980, 1982; Liberman and Oliver, 1984). However, it has to be mentioned that in mice, presynaptic ribbons are distributed rather uniformly at the IHC base (Meyer et al., 2009). Further, compared to cats, the SR distribution of mouse ANFs is not clearly bimodal but rather uniform (Taberner and Liberman, 2005). (3) Interestingly, the innervation pattern of SGNs to the IHC and their response characteristics further correlated with IHC presynaptic AZ properties. Based on ultrastructural analysis, low/medium-SR SGNs innervate synapses with larger or multiple ribbons that contain a large number of SVs (Figure 7, left) (Merchan-Perez and Liberman, 1996). Live-cell confocal $\mathrm{Ca}^{2+}$-signals and simultaneously analyzed fluorescently labeled ribbons showed rather heterogeneous amplitudes within individual IHCs, representing a strong variance in both presynaptic ribbon size and synaptic $\mathrm{Ca}^{2+}$-influx amplitude (Frank et al., 2009; Ohn et al., 2016). Still, correlations could be found: larger ribbons had more synaptic $\mathrm{Ca}^{2+}$-channels, increased synaptic $\mathrm{Ca}^{2+}$-influx, and were located towards the modiolar IHC symmetry axis. AZs on the modiolar side of the IHCs are further characterized by on average a more depolarized voltage of half-maximal activation of $\mathrm{Ca}^{2+}$-influx ( $\mathrm{V}_{\text {half }}$ ) (Frank et al., 2009; Meyer et al., 2009; Ohn et al., 2016). 
Thus, the state-of-the-art working hypothesis is that synapses on the modiolar side elicit less spontaneous SV release due to their more depolarized $\mathrm{Ca}^{2+}$-channel $\mathrm{V}_{\text {half }}$ and require higher sound amplitudes to reach the threshold of release, which is in line with the response properties of low-SR SGNs. The larger number of SVs could enable them to increase the release of neurotransmitter over a wide range of sound intensities contributing to the large dynamic range of low-SR SGNs. On the other hand, synapses on the pillar side spontaneously release SVs with a higher rate due to their more hyperpolarized $\mathrm{Ca}^{2+}$ channel $V_{\text {half. }}$ Thereby, they are more sensitive to low sound pressure levels, which is again in line with the response characteristics of high-SR SGNs. Likely, this diversity in SGN response characteristics may be a potential mechanism to encode the sound intensity of a given sound stimulus over a broad range of sound pressure levels. However, a characterization of heterogeneous SGN firing properties - evoked by input from only a single IHC - remains to be demonstrated by comparing the postsynaptic responses of multiple SGNs contacting the same IHC.

\subsection{Aim of this work}

This work aims to elucidate the molecular physiology of synaptic transmission at the first auditory synapse, the IHC ribbon synapse.

The first aim was to characterize the role of RIM-BP2, a new candidate molecular linker between $\mathrm{Ca}^{2+}$-channels and SVs, to investigate how the highly specialized sound-stimulus coding of the IHCs is orchestrated in more detail. Therefore, multiple techniques such as confocal and STED immunofluorescence microscopy, electron microscopy, patch-clamp, and confocal $\mathrm{Ca}^{2+}$-imaging, as well as auditory systems physiology, were combined to explore the morphological and physiological effects of genetic RIM-BP2 disruption in constitutive RIM-BP2 knockout mice.

The second aim was to establish a readout-system for simultaneously monitoring multiple SGN responses upon presynaptic IHC stimulation. Recording postsynaptic spiking properties of several SGNs simultaneously addresses postsynaptic response diversity on a single synapse level with respect to the same depolarizing IHC stimulus. This may provide a functional link between heterogeneous presynaptic AZ properties and postsynaptic SGN spike response diversity, which might be a potential mechanism of sound intensity coding. Therefore, an experimental setup for live-cell voltage imaging and SGN specific expression of the GEVIs QuasAr2 and 3 in SGNs was established. 


\section{Materials and Methods}

\subsection{Mouse lines}

\section{RIM-BP2 knockout mouse line}

Grauel and colleagues generated the RIM-BP2 knockout mouse line (Grauel et al., 2016) using homozygous recombination, replacing exon 17 of the RIM-BP2 gene with a neomycincassette. The mouse line was kept in a heterozygous breeding. Littermate RIM-BP2 knockout $\left(R I M-B P 2^{-/}\right)$and $R I M-B P 2$ wild-type $\left(R I M-B P 2^{++}\right)$mice as well as wild-type C57BI/6 mice were used for experiments. Disruption of RIM-BP2 was confirmed by genotyping (Grauel et al., 2016). Mice of either sex were examined between postnatal day 14 (p14) and p16 for hair cell physiology and $\mathrm{Ca}^{2+}$-imaging; between day p20 and p23 for immunohistochemistry, immunofluorescence microscopy and electron tomography; and between p60 to p65 for auditory evoked ABRs, distortion product otoacoustic emissions (DPOAEs) and single unit recordings from ANFs. All experiments complied with national animal care guidelines and were approved by the University of Göttingen board for animal welfare and the animal welfare office of the state of Lower Saxony.

\section{Floxpatch and Bhlhb5-Cre mouse line}

Lou and colleagues generated the Cre-dependent transgenic Optopatch2 mouse line. This line, also called Floxpatch, allows optical stimulation and voltage readout of Cre-expressing cells at the same time. It was created by targeted knock-in of the Optopatch2 construct at the Rosa26 locus (Lou et al., 2016) (Figure 8A). The Optopatch2 construct is expressed under the ubiquitous CAG promoter and comprises QuasAr2-dark-mOrange2 (the GEVI QuasAr2 tagged with dark-mOrange2) and CheRiff-eGFP, the blue-shifted channelrhodopsin variant CheRiff tagged with eGFP, connected by a P2A sequence, a selfcleaving ribosomes skip sequence from porcine teschovirus-1 in order to stoichiometrically co-express QuasAr2-dark-mOrange2 and CheRiff-eGFP (Figure 8C). A premature stop codon flanked by loxP sites was inserted upstream of the Optopatch2 construct. A Bhlhb5Cre driver line (Ross et al., 2010) was crossed with the Floxpatch mouse line to excise the premature stop codon by Cre-mediated recombination and allow tissue specific expression of Optopatch2 (Figure 8B, C).

The laboratory of Prof. Adam Cohen provided Floxpatch mice. The laboratory of Prof. Lisa Goodrich provided Bhlhb5-Cre mice. Mice were kept in a homozygous (Floxpatch ${ }^{f / / f}$ ) $x$ heterozygous (Bh/hb5-Cre ${ }^{+-}$) breeding at the Harvard University; experiments employing 
Floxpatch-Bhlhb5-Cre mice were performed in the laboratory of Prof. Adam Cohen, Department of Chemistry and Chemical Biology, Harvard. Cre-positive and Cre-negative control mice of either sex were examined between $\mathrm{p} 14$ and $\mathrm{p} 16$.

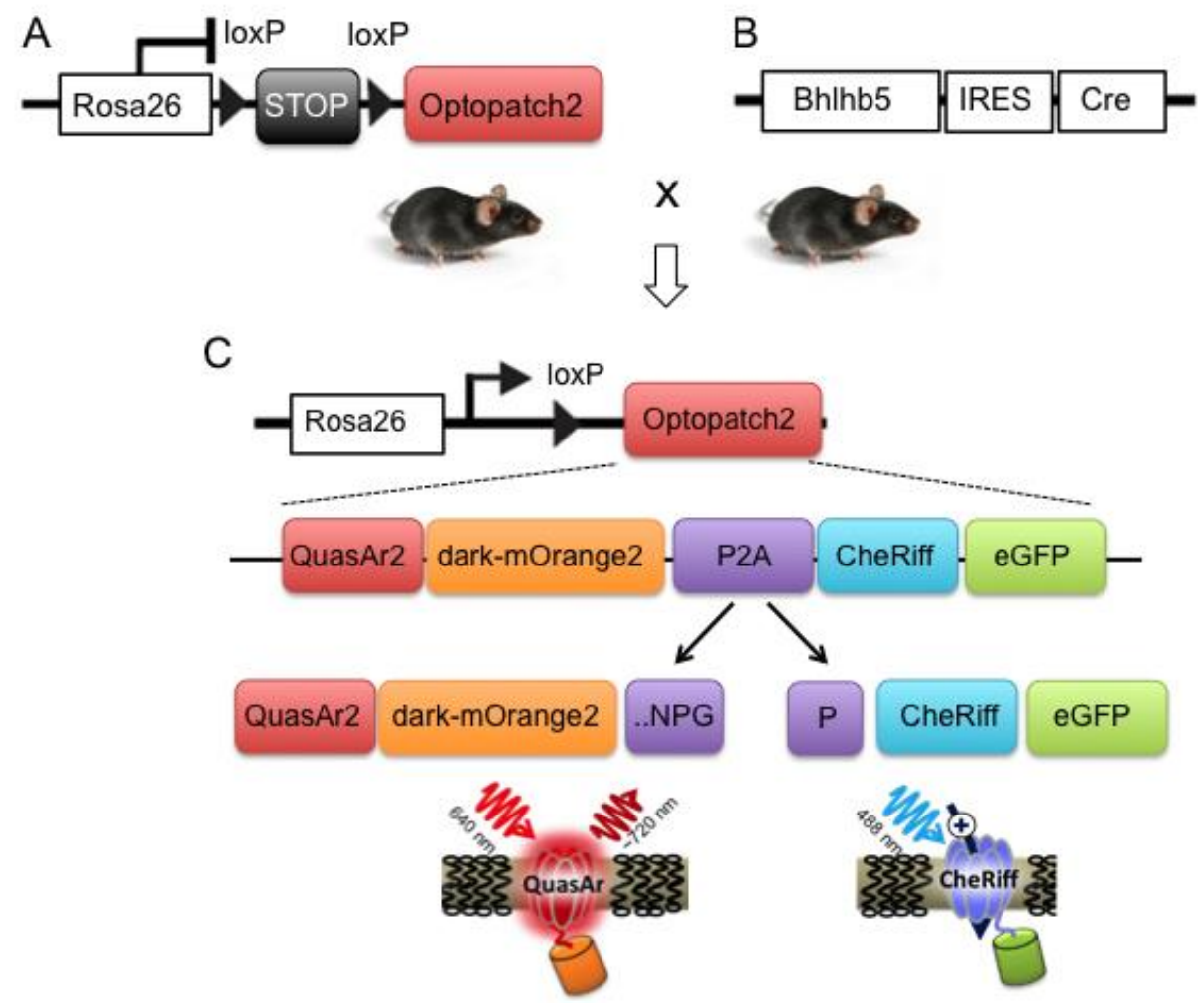

Figure 8: Floxpatch and Bhlhb5-Cre mouse line

[A] The Cre-dependent transgenic Floxpatch mouse line was created via knock-in of the Optopatch2 construct at the Rosa26 locus (Lou et al., 2016). [B] A Bhlhb5-Cre driver line mediates tissue specific excision of the premature stop codon upstream of Optopatch2 by Cre-mediated recombination (Ross et al., 2010). [C] Transcription of Optopatch2 leads to stoichiometric co-expression of QuasAr2-dark-mOrange2 and CheRiff-eGFP via the selfcleaving ribosomes skip sequence from porcine teschovirus-1, P2A. Figure partly adapted from Hochbaum et al. 2014.

\subsection{Viral optopatch construct and postnatal injection of AAV}

\section{Construct design}

The Optopatch3 construct (Figure 9) comprises the voltage indicator QuasAr3(Q95H) and the channelrhodopsin variant CheRiff and was designed and cloned by the laboratory of Prof. Adam Cohen (Harvard University, unpublished). In order to stoichiometrically coexpress QuasAr3(Q95H) and CheRiff, a self-cleaving ribosome skip sequence from porcine teschovirus-1 (P2A) was placed between the two genes (Hochbaum et al., 2014) in the same way as done in the Floxpatch mouse line (Lou et al., 2016). QuasAr3 was tagged with the fluorescent reporter Citrine. WPRE is the woodchuck hepatitis virus posttranscriptional 
regulatory element, TSX3 and TS are trafficking signal motifs, and ER2 is an endoplasmic reticulum (ER) export sequence (Hochbaum et al., 2014). The Optopatch3 construct was expressed under the human Synapsin promoter (H-Syn) and the expression cassette was flanked by inverted terminal repeats (ITR) of adeno-associated virus (AAV) 2. AAV with capsid proteins of serotype 6, hence AAV2/6, was produced by the University of North Carolina Vector Core facility (Titer: $2.1 \times 10^{12}$ vector genomes $/ \mathrm{mL}$ ). AAV2/6 and the $\mathrm{H}$-Syn were used to drive transgenic expression of QuasAr3-Citrine and CheRiff in SGNs.

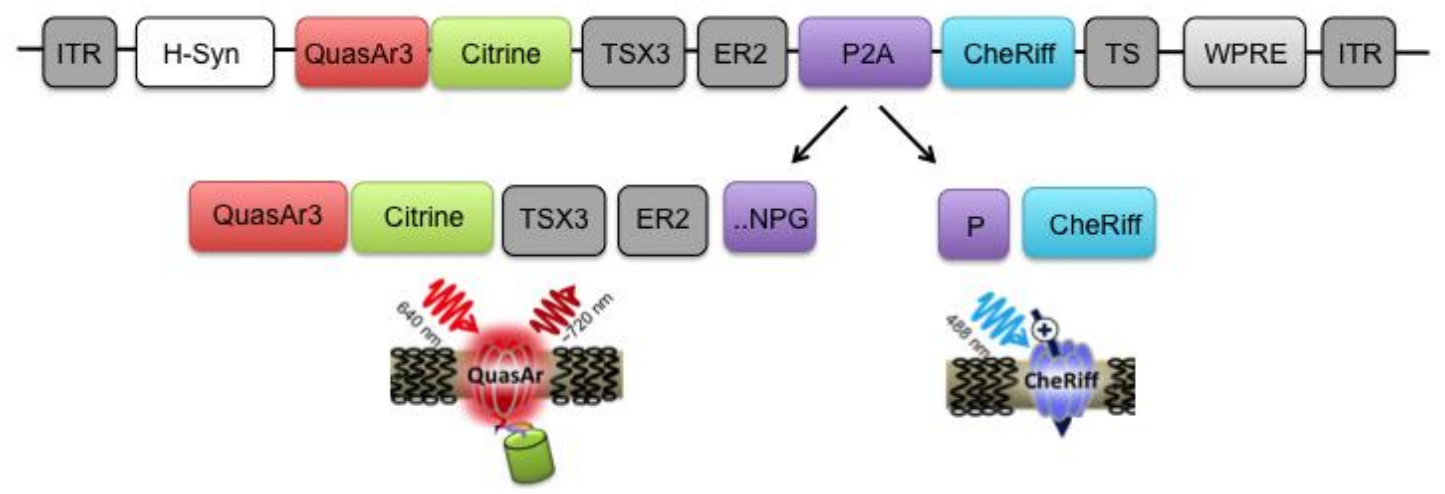

Figure 9: QuasAr3-CheRiff viral Optopatch3 construct design

The Optopatch3 construct contains QuasAr3-Citrine and CheRiff, linked by a self-cleaving ribosome skip sequence from porcine teschovirus-1 (P2A) for stoichiometric expression of QuasAr3 and CheRiff, as well as $H$-Syn to drive neuronal expression, woodchuck hepatitis virus posttranscriptional regulatory element (WPRE) to enhance transcription, trafficking signal motifs (TSX3 and TS), and an endoplasmic reticulum (ER) export sequence (ER2) to improve protein trafficking, and inverted terminal repeats (ITR) of AAV2/6. Figure partly adapted from Hochbaum et al. 2014.

\section{Postnatal injection of AAV}

Christiane Senger-Freitag injected AAV2/6 postnatally into the left ear's scala tympani of wild-type C57BI/6 mice via the round window. Experiments were performed at day p5 to p6 essentially as described in Akil et al. 2012. Briefly, mice were anesthetized by application of isoflurane (5\% for 2-3 min, maintained at 2-3\%) and depth of anesthesia was continuously checked by response to toe pinch. Preoperatively, animals were given subcutaneous injections of carprofen $(5 \mathrm{mg} / \mathrm{kg})$ und buprenorphin $(0.1 \mathrm{mg} / \mathrm{kg})$ to manage inflammation and pain. The left ear was approached via a ventral, paramedian incision in the neck and a hole was scraped into the bulla. 1-3 $\mu \mathrm{L}$ of AAV2/6 were injected into the cochlear perilymph with a hydraulic pump. The bulla was quickly sealed with fascia and adipose tissue and the wound was sutured. Mice were placed into a separate cage, where temperature was maintained at $37^{\circ} \mathrm{C}$ with a heat plate. Animals were closely monitored and put back to their home-cages after awakening from their anesthesia. 


\subsection{Whole-mount preparations of the organ of Corti}

Mice were anesthetized with $\mathrm{CO}_{2}$ and decapitated. After removing the skin, the skull was hemisected along the sagittal midline and placed in Petri dishes filled with HEPES-buffered Hanks' balanced salt solution (142 mM NaCl, $5.4 \mathrm{mM} \mathrm{KCl}, 1.0 \mathrm{mM} \mathrm{MgCl}_{2}, 0.5 \mathrm{mM} \mathrm{MgSO}_{4}$, $0.1 \mathrm{mM} \mathrm{CaCl}_{2}, 3.4 \mathrm{mM}$ L-Glutamine, $11 \mathrm{mM}$ D-Glucose, $10 \mathrm{mM} \mathrm{HEPES}$, pH adjusted to 7.3 $(\mathrm{NaOH})$, osmolarity 300 mOsm, all chemicals were obtained from Sigma-Aldrich). Under a dissection microscope the brain was removed and the cochlea was separated from the temporal bones by carefully twisting it out of the skull with a pair of medium fine forceps. Using fine forceps, the bony shell of the apical turn of the cochlea was opened to expose the apical coil of the organ of Corti. The stria vascularis was gently removed by unwinding it with very fine forceps and the first turn of the organ of Corti was separated from the modiolus.

\subsection{Patch-clamp recordings and confocal $\mathrm{Ca}^{2+}$-imaging}

For transfer of the organ of Corti whole-mount preparation to a recording chamber, a firepolished Pateur's pipette was used. For fastening the dissected organ of Corti to the chamber bottom, a grid of nylon threads was placed onto the organ of Corti, which was placed with the hair cells' stereocilia facing upwards. To allow access to a clean IHC membrane, all cells facing the pillar/abneural side of the organ of Corti were cleaned away. First, the tectorial membrane was removed with a wide-mouthed cleaning pipette (tip diameter of $\sim 20-30 \mu \mathrm{m}$ ). Then, perfusion with modified Ringer's solution (containing (in $\mathrm{mM}$ ): 104 or $110 \mathrm{NaCl}, 35 \mathrm{TEA}-\mathrm{Cl}, 2.8 \mathrm{KCl}, 2$ or $5 \mathrm{CaCl}_{2}, 1 \mathrm{MgCl}_{2}, 10 \mathrm{NaOH}$-HEPES, 11.3 D-glucose, $\mathrm{pH} 7.3$, osmolarity $\sim 300 \mathrm{mOsm}$, all chemicals were obtained from SigmaAldrich) was started with an exchange rate of approximately 0.5 to $1 \mathrm{~mL} / \mathrm{min}$. OHCs and outer and inner pillar cells were removed by sucking in the nuclei with a smaller cleaning pipette (tip diameter of $\sim 2-5 \mu \mathrm{m}$ ), waiting until cell death and finally sucking in the residual cell debris with very low pressure and minimal mechanical force. In the same way, but with even smaller pipettes (tip diameter of $\sim 2 \mu \mathrm{m}$ ) phalangeal cells were cleaned off last. Cleaning pipettes were pulled from soda-lime glass capillaries $(1.5 \times 80 \mathrm{~mm}, 0.15 \mathrm{~mm}$ wall thickness, Hilgenberg, Germany) with a vertical puller (Patch-Pipette Puller PA-10, E.S.F. Electronic, Göttingen, Germany). Patch pipettes were pulled from borosilicate glass capillaries (GB150F-8P or GB150-8P: capillaries with or without filament for ruptured or perforated patch-clamp recordings, respectively; $0.86 \times 1.50 \times 80 \mathrm{~mm}$; Science Products, Hofheim, Germany) with a horizontal Sutter P-97 Flaming/Brown Micropipette puller (Sutter 
Instruments Company, Novato, USA) (Table 1). The pipette-tip was fire-polished with a custom-made microforge to enhance sealing with the IHC plasma membrane.

Table 1: Puller program for patch pipettes

\begin{tabular}{|l|l|l|l|l|l|}
\hline Line & Heat & Filament & Velocity & Delay & Pull \\
\hline $\mathbf{1}$ & 640 & 4 & 30 & 200 & 20 \\
\hline $\mathbf{2}$ & 630 & 4 & 25 & 200 & \\
\hline $\mathbf{3}$ & 620 & 4 & 20 & 200 & \\
\hline $\mathbf{4}$ & 620 & 4 & 20 & 200 & 15 \\
\hline $\mathbf{5}$ & 625 & 4 & 25 & 200 & 30 \\
\hline
\end{tabular}

Diverse patch-clamp setups equipped with BX50WI microscopes (Olympus, Hamburg, Germany), 60x magnification objectives (0.9 NA, water immersion objective, Olympus), shielded by Faraday cages and assembled on hydraulic air tables (TMC, Peabody, USA) were used for the cellular electrophysiology. Fluid level in the chamber as well as pipette pressure were controlled by MPCU-3 pumps (Lorenz Messgeätebau, Kathlenburg-Lindau, Germany). Piezoelectric micromanipulators (MP-285, Sutter Instruments Company, Novato, USA) were used for moving cleaning and patch-pipettes nearby the whole-mount preparation. For patch-clamp measurements EPC-10 USB amplifiers (HEKA Elektronik, Lambrecht, Germany) controlled by Patchmaster software (HEKA Elektronik, Lambrecht, Germany) were used.

Patch-clamp recordings of IHCs from freshly dissected organs of Corti were carried out as described by Moser and Beutner 2000. For perforated-patch recordings of whole-cell $\mathrm{Ca}^{2+}-$ current and exocytosis, recording pipettes were dipped into amphotericin B (pore-forming antibiotic) free intracellular solution for $50 \mathrm{~s}$ to avoid premature amphotericin $\mathrm{B}$ exposure of the tissue and filled with intracellular solution containing (in $\mathrm{mM}$ ): 130 Cs-gluconate, 10 TEA-Cl, 10 4-AP, 10 HEPES, $1 \mathrm{MgCl}_{2}$, amphotericin B (300 $\mathrm{gg} / \mathrm{ml}$, Calbiochem), pH 7.2 (osmolarity 290 mOsm, all chemicals were obtained from Sigma-Aldrich). Ruptured-patch whole-cell recordings were performed for confocal $\mathrm{Ca}^{2+}$-imaging with the solution described above excluding amphotericin B, but including (in mM) 4 Mg-ATP, 0.3 Na-GTP, 1 EGTA, 0.4 Fluo-4FF (Invitrogen) and 0.01 carboxytetramethylrhodamine (TAMRA)-conjugated dimeric RIBEYE-binding peptide (Francis et al., 2011). Recording pipettes with resistances of 4.5 to 7.0 M $\Omega$ (when filled with Cs-based intracellular solution) were advanced towards the IHC under positive pressure of $\sim 2.5 \mathrm{~cm} \mathrm{H} \mathrm{H}_{2} \mathrm{O}$. After approaching the IHC, the positive pressure was released to form a gigaseal (with electrical resistance in $G \Omega$ ) between the patch pipette and the IHC's membrane. The IHC was immediately voltage-clamped at a 
holding potential of $-84 \mathrm{mV}$. For pipette capacitance compensation the EPC-10's built-in auto $\mathrm{C}_{\text {fast }}$ compensation function was used to eliminate fast transient currents. Whole-cell patch-clamp configuration was achieved by two different approaches. In case of confocal $\mathrm{Ca}^{2+}$-imaging, the IHC membrane was ruptured by a short, negative pressure pulse through the suction tube. In case of whole-cell $\mathrm{Ca}^{2+}$-current and exocytosis recordings, the IHC membrane was perforated by amphotericin B inside the intracellular solution. Slow current transients, resulting from the whole-cell membrane capacitance were compensated using the EPC-10's built-in auto $\mathrm{C}_{\text {slow }}$ compensation function. All currents were leak-corrected using a $\mathrm{p} / \mathrm{n}(0.25 / 8)$ protocol, with a $\mathrm{p} / \mathrm{n}$ holding potential of $-90 \mathrm{mV}$, low pass filtered at 2.9 $\mathrm{kHz}$ and sampled at $100 \mathrm{kHz}$. IHCs with leak current greater than $-30 \mathrm{pA}$ or $\mathrm{R}_{\mathrm{s}}$ greater than $30 \mathrm{M} \Omega$ were excluded from the analysis. In the offline analysis using Igor Pro software (Wavemetrics, Lake Oswego, USA), all voltages were corrected for liquid-junction potentials (LJP $-14 \mathrm{mV}$ ) and for the voltage drop across the access resistance to the cell (series resistance, $\left.R_{S}\right)$.

\section{Analysis of membrane capacitance}

For membrane capacitance $\left(\mathrm{C}_{\mathrm{m}}\right)$ measurements, IHCs were stimulated by depolarizations of different durations to $-14 \mathrm{mV}$ at intervals of 30 to $60 \mathrm{~s}$. Exocytic $\mathrm{C}_{\mathrm{m}}$ changes $\left(\Delta \mathrm{C}_{\mathrm{m}}\right)$ were measured using the Lindau-Neher technique (Lindau and Neher, 1988), as previously described (Moser and Beutner, 2000) (sinewave frequency $1 \mathrm{kHz}$, sinewave $70 \mathrm{mV}$ peakto-peak, $V_{\text {reversal }}-45 \mathrm{mV}$ ). $\Delta \mathrm{C}_{\mathrm{m}}$ was quantified as the averaged (over $400 \mathrm{~ms}$ ) difference between $\mathrm{C}_{\mathrm{m}}$ before and after (skipping the first $100 \mathrm{~ms}$ ) $\mathrm{IHC}$ depolarization. Mean $\Delta \mathrm{C}_{\mathrm{m}}$ estimates present grand averages calculated from the mean estimates of individual IHCs (2-3 sweeps per stimulus per IHC) to avoid dominance of IHCs contributing more sweeps.

\section{Analysis of $\mathrm{Ca}^{2+}$ current-voltage relationships}

Current-voltage relationships (IVs) of $\mathrm{IHC} \mathrm{Ca}{ }^{2+}$-currents were calculated from the last $8 \mathrm{~ms}$ of currents evoked by $10 \mathrm{~ms}$ step depolarizations to various potentials from $-100 \mathrm{mV}$ to +30 $\mathrm{mV}$ in $5 \mathrm{mV}$ increments. Calculating the conductance of the cells and plotting it against the potential to which the cell was clamped created fractional activation curves:

$$
G(V)=\frac{I_{C a}}{\left(V-V_{\text {rev }}\right)} \quad \text { (Equation 1) }
$$

Here $G$ is the conductance, $I_{C a}$ the $\mathrm{Ca}^{2+}$-current, $V$ the potential to which the cell was clamped and $V_{\text {rev }}$ the reversal potential of the current obtained by fitting a line to the IV from 6 to $26 \mathrm{mV}$ and extrapolating it to the potential of zero current. 
The traces were normalized to the maximum conductance $\left(G_{n}\right)$ calculated in the range of 20 to $+10 \mathrm{mV}$ and fitted with a Boltzmann equation:

$$
G_{n}(V)=\frac{G_{n, \text { max }}}{1+e^{\frac{\text { hallf-V }}{k}}} \quad \text { (Equation 2) }
$$

Here $G_{n, \max }$ is the maximal conductance, $V$ the potential to which the cell was clamped, $V_{\text {half }}$ the voltage of half-maximal activation, and $k$ the slope of activation.

\section{Analysis of $\mathrm{Ca}^{2+}$ inactivation kinetics}

The $\mathrm{Ca}^{2+}$-current $\left(I_{C a}\right)$ inactivation time constant $\tau$ was measured by fitting an exponential function to the last $195 \mathrm{~ms}$ of $I_{C a}$ traces during $200 \mathrm{~ms}$ depolarizations to $-14 \mathrm{mV}$.

$$
y=A \times e^{(- \text {invTaux })} \quad(\text { Equation 3) }
$$

Here $A$ is the $I_{C a}$ amplitude, invTau is the inverse of the time constant $\tau$.

\section{Estimating the apparent $\mathrm{Ca}^{2+}$ cooperativity of exocytosis}

The coupling between $\mathrm{Ca}^{2+}$-channels and SVs was addressed essentially as described in Wong et al. 2014, by calculating the apparent $\mathrm{Ca}^{2+}$ cooperativity $m$ of exocytosis. During perforated patch recordings, exocytic membrane capacitance changes $\left(\Delta \mathrm{C}_{\mathrm{m}}\right)$ and corresponding whole-cell $\mathrm{Ca}^{2+}$-currents $\left(I_{C a}\right)$ were measured during 20 ms depolarizations (to $-14 \mathrm{mV}$ ) to primarily recruit the RRP of vesicles (Beutner and Moser, 2001; Moser and Beutner, 2000). The number of open $\mathrm{Ca}^{2+}$-channels of the IHC was gradually reduced by slow perfusion $(0.5 \mathrm{~mL} / \mathrm{min})$ with the L-type $\mathrm{Ca}^{2+}$-channel antagonist isradipine $(10 \mu \mathrm{M})$ in the extracellular solution $\left(5 \mathrm{mM} \mathrm{CaCl}_{2}\right.$ ). The integrated $I_{C a}$ charge was plotted against its respective $\Delta \mathrm{C}_{\mathrm{m}}$ on double-logarithmic axes and data points of each IHC were fit with a power function:

$$
\Delta C_{m}=A \times\left(Q_{C a}\right)^{m} \quad(\text { Equation 4) }
$$

Here $\Delta C_{m}$ is the exocytic membrane capacitance change, $Q_{C a}$ the whole-cell $\mathrm{Ca}^{2+}$-current integral and the power $m$ describing the apparent $\mathrm{Ca}^{2+}$ cooperativity of SV release. 


\section{Confocal $\mathrm{Ca}^{2+}$-imaging}

Confocal $\mathrm{Ca}^{2+}$-imaging was carried out essentially as described by Frank et al. 2009. Experiments were performed on an upright BX50WI microscope (Olympus, Hamburg, Germany) equipped with a confocal laser scanning unit (Olympus Fluoview 300 confocal scanner, Olympus, Hamburg, Germany), a $50 \mathrm{~mW}, 488 \mathrm{~nm}$ solid state excitation laser (0.5 \% laser power, Cyan, Newport Spectra-Physics, Santa Clara, USA), a $1.5 \mathrm{~mW}, 543 \mathrm{~nm} \mathrm{He}-$ Ne excitation laser (5 \% laser power, LGK 7786 P150, Lasos, Jena, Germany), LUMPlan 60x magnification NA 0.9 objective (Olympus) and two photomultiplier tubes (Gain $700 \mathrm{~V}$, Hamamatsu, Herrsching am Ammersee, Germany) as detectors. The microscope was controlled by Olympus Fluoview acquisition software and triggered via Patchmaster software (HEKA Elektronik, Lambrecht, Germany) for synchronization with whole-cell patch-clamp recordings.

Individual IHC AZs were identified by fluorescence of synaptic ribbons labeled with TAMRAconjugated dimeric RIBEYE-binding peptide (Francis et al., 2011) $(0.01 \mathrm{mM}$ in the intracellular solution). Confocal line-scans $(0.7 \mathrm{kHz})$ through the center of the synaptic ribbon were performed during short $\mathrm{IHC}$ step depolarizations (20 ms to $-7 \mathrm{mV}$ ) and $\mathrm{Ca}^{2+}$ indicator fluorescence increase (Fluo-4FF, $0.4 \mathrm{mM}$ ) was measured as readout of synaptic $\mathrm{Ca}^{2+}$-influx. For quantification of synaptic $\mathrm{Ca}^{2+}$-signal amplitudes, the fluorescence change $(\Delta \mathrm{F})$ was calculated as the average evoked fluorescence intensity change for 3 peakcentered pixels over the last $10 \mathrm{~ms}$ of depolarization (from 3 averaged line-scans). For normalization $\left(\Delta \mathrm{F} / \mathrm{F}_{0}\right)$, synaptic $\mathrm{Ca}^{2+}$-signals $(\Delta \mathrm{F})$ from line-scans were divided by their baseline fluorescence $\left(\mathrm{F}_{0}\right)$. The spatial spread of $\mathrm{Ca}^{2+}$-signals was estimated by fitting a Gaussian function to the individual $\mathrm{Ca}^{2+}$-signal line profiles to obtain full width at half maximum (FWHM) estimates:

$$
\begin{aligned}
& f(x)=y_{0}+A \times e^{-\frac{\left(x-x_{0}\right)^{2}}{w_{i d t h}^{2}}} \quad \text { (Equation 5) } \\
& F W H M=\text { width } \times 2 \times \sqrt{\ln 2} \text { (Equation 6) }
\end{aligned}
$$

Here, $y_{0}$ is the baseline fluorescence, $A$ is the amplitude, $x_{0}$ is the center of the Gaussian distribution and width is its width. 


\subsection{Wide field voltage imaging}

\section{Microscope and hardware}

Voltage imaging was carried out with a custom-built wide field imaging microscope (Figure 10): Wide field illumination was achieved by expansion of a diode laser beam (labeled "S" in the figure, OBIS 637 nm LX 140 mW diode laser; Coherent Inc., Santa Clara, USA) using a plan-concave lens (“L1”) and a plan-convex lens (“L2”). To gain a more uniform Gaussian laser beam profile, an iris ("Iris") was used (L1, L2, Iris: Thorlabs, Munich, Germany). To couple the laser beam into the microscope an excitation filter (“F1", 635/20 nm bandpass; Chroma Technology Corp., Vermont, USA) and dichroic mirror (“DM”, Notch-dichroic beamsplitter 633/70 nm; AHF Analysentechnik, Tübingen, Germany) was mounted into a dual port 1X C-mount (U-DP/U-DP1CC; Olympus, Hamburg, Germany). An achromatic lens ("L3"; Thorlabs, Munich, Germany) focused the collimated laser beam onto the back-focal plane of the objective ("Objective", LUMPlan 60x magnification NA 0.9 water immersion; Olympus, Hamburg, Germany) to create a parallel laser beam simultaneously illuminating a relatively large area of the tissue. Lenses were fixed in a cage system $(60 \mathrm{~mm})$ mounted on a breadboard (Thorlabs, Munich, Germany). Fluorescence emission was collected through the same objective. Before imaging it onto the camera chip ("sCMOS Camera", Orca-Flash4.0V2 sCMOS Camera; Hamamatsu Photonics Deutschland GmbH, Herrsching am Ammersee, Germany), it passed back through the dichroic mirror ("DM"), an emission filter ("F2", 655 nm longpass; Chroma Technology Corp., Vermont, USA), tube lens ("T") and post-magnification lenses (“N”) (Olympus, Hamburg, Germany).

An EPC-10 USB amplifier (HEKA Elektronik, Lambrecht, Germany) controlled by Patchmaster software (HEKA Elektronik, Lambrecht, Germany) was used as master to control the laser and camera. The laser was regulated via digital modulation (digital-current mode) with the Coherent Connection software (Coherent Inc., Santa Clara, USA). The camera was controlled with a digital start trigger (start trigger mode) with the Hokawo2.6 software (Hamamatsu Photonics Deutschland $\mathrm{GmbH}$, Herrsching am Ammersee, Germany). The "start trigger mode" is an external control mode, where, following the start trigger signal, the camera is operated in internal trigger mode enabling continuous imaging at the highest frame rate. The image acquisition rate during voltage imaging was 1000 frames/s using $2 \times 2$ pixel binning of a $2048 \times 184$ pixel area (pixel size: $6.5 \mu \mathrm{m} \times 6.5 \mu \mathrm{m}$ ), which was set symmetrically to the center-line of the camera chip. 


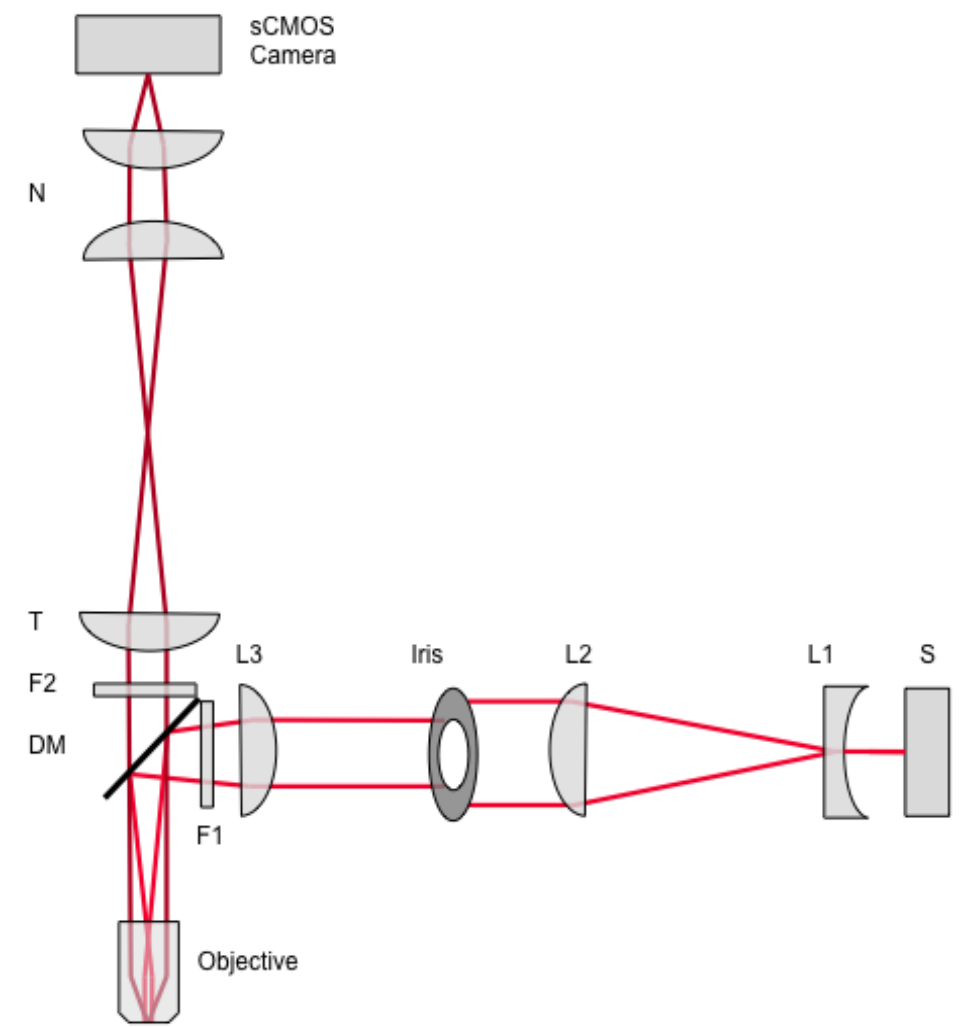

Figure 10: Diagram of custom-built wide field microscope

Illumination source: (S) OBIS $637 \mathrm{~nm}$ LX $140 \mathrm{~mW}$ diode laser (Coherent Inc., Santa Clara, USA); Lenses: ( $L 1)$ plan-concave lens ( $f=-6 \mathrm{~mm}),(L 2)$ plan-convex lens $(f=150 \mathrm{~mm}),(L 3)$ achromatic lens ( $f=200 \mathrm{~mm}),(\mathrm{L} 1-3$ : Thorlabs, Munich, Germany); $(T)$ tube lens $(f=180 \mathrm{~mm})$, (N) post-magnification lenses, (T and L: Olympus, Hamburg, Germany), Dichroic mirror: (DM) Notch-dichroic beamsplitter 633/70 nm (AHF Analysentechnik, Tübingen, Germany), Filters: (F1) 635/20 nm bandpass, (F2) 655 nm longpass, (F1 and F2: Chroma Technology Corp., Vermont, USA), Objective: LUMPlan 60x magnification NA 0.9 water immersion objective (Olympus, Hamburg, Germany), Detector: Orca-Flash4.0V2 sCMOS Camera (Hamamatsu Photonics Deutschland GmbH, Herrsching am Ammersee, Germany).

\section{Optogenetic and patch-clamp stimulation}

Voltage imaging with the reporter QuasAr2 was performed on a custom-built wide-field microscope described in Kralj et al. 2011 and Hochbaum et al. 2014 in the laboratory of Prof. Adam Cohen, Harvard University. SGNs were stimulated optically, using 500 ms pulses of $488 \mathrm{~nm}$ wavelength to induce photocurrent through activation of CheRiff (Hochbaum et al., 2014; Lou et al., 2016). Voltage imaging with the reporter QuasAr3 was performed on the custom-built wide field microscope described above (Figure 10). After postnatal AAV2/6 injection, (see chapter 3.2) organs of Corti were dissected at age p14-16 and placed in the recording chamber in modified Ringer's solution. Two different stimulation paradigms were used: (1) IHCs were patch-clamped (see chapter 3.4) and depolarized to $-14 \mathrm{mV}$ for various durations to induce presynaptic neurotransmitter release onto the postsynaptic SGNs, resulting in afferent activity. IHCs were patched in the perforated patch- 
clamp configuration and whole cell $\mathrm{Ca}^{2+}$-current and exocytic capacitance changes were measured. Simultaneously, QuasAr3 fluorescence was recorded before, during, and after IHC depolarization from several boutons targeting the stimulated IHC. (2) Alternatively, optogenetic stimulation of SGNs was performed by $473 \mathrm{~nm}$ laser illumination through an optic fiber (Laser: MBL-473-(FC), $50 \mathrm{~mW}$, Changchun New Industries Optoelectronics Tech. Co., Ltd., China; Fiber: M42L05 - $\varnothing 50 \mu \mathrm{m}, 0.22$ NA, Thorlabs; Munich, Germany). The fiber was fixed on a piezoelectric micromanipulator (MP-285, Sutter Instruments Company, Novato, USA) and placed at approximately $250 \mu \mathrm{m}$ distance from the SGN boutons. $50 \mathrm{~ms}$ laser pulses of increasing intensity (1 to $15 \mathrm{~mW}$ ) were used to induce photocurrent through activation of CheRiff.

\section{Analysis}

QuasAr3 fluorescence was analyzed by choosing regions of interest (ROls) at the location of SGN boutons adjacent to the IHC. $\Delta \mathrm{F}_{\mathrm{RO}} / \mathrm{F}_{0}$ was calculated by first subtracting and and then dividing the mean fluorescence of the ROls over all time points $\left(F_{0}\right)$ from the mean ROI fluorescence of each time point $\left(\mathrm{F}_{\mathrm{RO}}\right)$. Analysis was performed using $\mathrm{NIH}$ ImageJ (Schneider et al., 2012) and Igor Pro software (Wavemetrics, Lake Oswego, USA). QuasAr2 fluorescence intensity was analyzed as described in Hochbaum et al., 2014. Briefly, ROls were manually chosen and the fluorescence was calculated with equal pixel weighting and no background subtraction or correction for photobleaching within individual ROls in Matlab software (Mathworks).

\subsection{Immunohistochemistry, confocal and STED microscopy}

\section{Cryo-sectioning of the cochlea}

The cochlea was prepared as described in 3.3. The bone above the apical turn of the cochlea was opened and the cochlea was fixed for 1 hour in $4 \%$ formaldehyde (FA) in phosphate buffered saline (PBS). Subsequently, the cochlea was washed in PBS for 1 day, decalcified in Ethylenediaminetetraacetic acid (EDTA) (120 mM, pH 7.0) for 3 days and incubated in sucrose (25\% in PBS) for 3 days. The cochlea was transferred into Cryomatrix (Thermo Fisher Scientific, Massachusetts, USA) filled aluminum chambers and the modiolus was oriented horizontally before rapid freezing in liquid nitrogen. Samples were stored at $-80^{\circ} \mathrm{C}$ until $16 \mu \mathrm{m}$ thick cryo-sections were cut with a cryostat (2800 Frigocut $\mathrm{E}$, Reichert-Jung Mannheim, Germany) at $-25^{\circ} \mathrm{C}$ and mounted onto Superfrost Plus microscope glass slides (Menzel, Braunschweig, Germany). Samples were dried at room temperature and stored at $-20^{\circ} \mathrm{C}$. 


\section{Immunohistochemistry}

Immunohistochemistry was essentially performed as described in Khimich et al. 2005. Freshly dissected apical turns of the organs of Corti were either fixed with 4\% FA in PBS on ice for 10 minutes or with methanol at $-20^{\circ} \mathrm{C}$ for 20 minutes. Thereafter, the whole-mount preparations or cryo-samples were washed $3 \times 10$ min in PBS and incubated for 1 hour in goat serum dilution buffer (GSDB: 16\% normal goat serum, $450 \mathrm{mM} \mathrm{NaCl}, 0.3 \%$ Triton X100, $20 \mathrm{mM}$ phosphate buffer, $\mathrm{pH}$ 7.4) in a wet chamber at room temperature. Primary antibodies (Table 2) were diluted in GSDB and applied overnight at $4^{\circ} \mathrm{C}$ in a wet chamber.

Table 2: Antibodies and dilutions used in immunohistochemistry

\begin{tabular}{|c|c|c|c|}
\hline Antibody & Dilution & Source & Provider \\
\hline anti-Bassoon & $1: 500$ & Guinea pig & $\begin{array}{l}\text { Synaptic Systems, Göttingen, } \\
\text { Germany }\end{array}$ \\
\hline anti-Calretinin (CR7697) & $1: 2000$ & Rabbit & Swant, Marly, Switzerland \\
\hline anti-Cav1.3 & $1: 50$ & Rabbit & $\begin{array}{l}\text { Alomone Labs, Jerusalem, } \\
\text { Israel }\end{array}$ \\
\hline anti-CtBP2 & $1: 200$ & Mouse & $\begin{array}{l}\text { BD Biosciences, San Jose, } \\
\text { USA }\end{array}$ \\
\hline anti-GFP & $1: 500$ & Mouse & $\begin{array}{l}\text { Abcam, Cambridge, United } \\
\text { Kingdom }\end{array}$ \\
\hline anti-GluR 2/3 & $1: 200$ & Rabbit & Chemicon, Billerica, USA \\
\hline anti-RIM-BP2 & $1: 200$ & Rabbit & $\begin{array}{l}\text { Synaptic Systems, Göttingen, } \\
\text { Germany }\end{array}$ \\
\hline Synapsin $1 / 2$ & $1: 500$ & Guinea pig & $\begin{array}{l}\text { Synaptic Systems, Göttingen, } \\
\text { Germany }\end{array}$ \\
\hline AlexaFluor488 anti-chicken & $1: 200$ & Goat & $\begin{array}{l}\text { Invitrogen, Karlsruhe, } \\
\text { Germany }\end{array}$ \\
\hline AlexaFluor568 anti-mouse & $1: 200$ & Goat & $\begin{array}{l}\text { Invitrogen, Karlsruhe, } \\
\text { Germany }\end{array}$ \\
\hline AlexaFluor647 anti-rabbit & $1: 200$ & Goat & $\begin{array}{l}\text { Invitrogen, Karlsruhe, } \\
\text { Germany }\end{array}$ \\
\hline Star580 anti-mouse & $1: 50$ & Goat & $\begin{array}{l}\text { Abberior Instruments } \mathrm{GmbH} \text {, } \\
\text { Göttingen, Germany }\end{array}$ \\
\hline Star635P anti-guinea pig & $1: 50$ & Goat & $\begin{array}{l}\text { Abberior Instruments } \mathrm{GmbH} \text {, } \\
\text { Göttingen, Germany }\end{array}$ \\
\hline Star635P anti-rabbit & $1: 50$ & Goat & $\begin{array}{l}\text { Abberior Instruments } \mathrm{GmbH} \text {, } \\
\text { Göttingen, Germany }\end{array}$ \\
\hline
\end{tabular}


After washing 3x10 min (wash buffer: $450 \mathrm{mM} \mathrm{NaCl}, 20 \mathrm{mM}$ phosphate buffer, $0.3 \%$ Triton $X-100$ ) the tissue was incubated with secondary antibodies (Table 2 ) in GSDB in a wet lightprotected chamber for 1 hour at room temperature. Then, the samples were washed $3 \times 10$ min in wash buffer and $1 \times 10 \mathrm{~min}$ in $5 \mathrm{mM}$ phosphate buffer, placed onto the glass microscopy slides with a drop of fluorescence mounting medium (Mowiol) and covered with thin glass coverslips.

\section{Fluorescence microscopy and data analysis}

Confocal images were acquired using a laser scanning confocal microscope (Leica TCS SP5, Leica Microsystems GmbH, Mannheim, Germany) equipped with 488, 561, and 633 $\mathrm{nm}$ lasers for excitation and a $63 \mathrm{x}$ oil immersion objective (1.4 NA, Leica) and 40x oil immersion objective (1.4 NA, Leica) (Facility for Innovative Light Microscopy (FILM), MaxPlanck-Institute for Biophysical Chemistry, Göttingen). STED images were acquired using a 2-color STED microscope (Abberior Instruments, Göttingen, Germany) equipped with 561 $\mathrm{nm}$ and $640 \mathrm{~nm}$ excitation lasers, a $775 \mathrm{~nm}$ laser for stimulated emission depletion (1.2 W) and a 100x oil immersion objective (1.4 NA, Olympus). Samples were treated in parallel and images were acquired in parallel, using same laser power, gain, and microscope settings.

Images were analyzed using NIH ImageJ (Schneider et al., 2012), Igor Pro software (Wavemetrics, Lake Oswego, USA), OriginPro software, and Imaris software (using custom MATLAB code by Gerhard Hoch) and assembled for display in Adobe Illustrator software (Adobe Systems Inc., San Jose, USA). STED images of $\mathrm{Ca}^{2+}$-channel and bassoon clusters were fitted with a 2-dimensional (2D) Gauss function using a genetic fit algorithm (Rio and Pareschi, 2001) to the subarea of the $X Y$ image containing the $\mathrm{Ca}^{2+}$-channel or bassoon clusters to obtain FWHM estimates for the long and short axes of the clusters. To quantify the size and fluorescence intensity of the fitted clusters, the area and integral (volume) below the 2D Gaussian fit were calculated:

$$
\begin{aligned}
& A=\pi \times \frac{F W H M_{\text {short }}}{2} \times \frac{F W H M_{\text {long }}}{2} \\
& V=2 \pi \times A m p \times \frac{F W H M_{\text {short }}}{2 \times \sqrt{2 \times \ln 2}} \times \frac{F W H M_{\text {long }}}{2 \times \sqrt{2 \times \ln 2}}
\end{aligned}
$$

(Equation 7)

(Equation 8)

Here $A$ is the area of the ellipse formed by the long and short axes of the 2D Gaussian fit, $V$ is the volume under the 2D Gaussian fit function, $A m p$ is its amplitude, $F W H M_{\text {short }}$ and FWHMlong are the FWHM of the short and long axis, respectively. 


\subsection{Electron tomography}

Prof. Carolin Wichmann performed electron tomography and generated tomograms. Embedding was carried out as described previously in Wong et al. 2014. Organ of Corti whole-mounts were fixed for $1 \mathrm{~h}$ on ice with $4 \%$ paraformaldehyde (PFA) and $0.5 \%$ glutaraldehyde in $1 \times \mathrm{PBS}, \mathrm{pH} 7.2$, followed by a fixation step with $2 \%$ glutaraldehyde in 0.1 $\mathrm{M}$ sodium cacodylate buffer, $\mathrm{pH} 7.2$, overnight on ice. The samples were washed 3 times in sodium cacodylate buffer, postfixed for $1 \mathrm{~h}$ with $1 \%$ osmium tetroxide $((\mathrm{v} / \mathrm{v})$ in $0.1 \mathrm{M}$ sodium cacodylate buffer), washed 3 times for $20 \mathrm{~min}$ in sodium cacodylate buffer followed by 3 brief washing steps in distilled water. All steps were performed on ice. The samples were stained en bloc with $1 \%(\mathrm{v} / \mathrm{v})$ uranyl acetate in distilled water for $1 \mathrm{~h}$ on ice and washed 3 times briefly with distilled water. Further, samples were dehydrated at room temperature in increasing ethanol concentrations, infiltrated in Epon resin $(100 \% \mathrm{EtOH} / \mathrm{Epon} 1: 1(\mathrm{v} / \mathrm{v})$, 30 and $90 \mathrm{~min} ; 100 \%$ Epon, overnight), and embedded for $48 \mathrm{~h}$ at $70^{\circ} \mathrm{C}$ in Epon resin. Following conventional embedding and trimming of the blocks, $250 \mathrm{~nm}$ sections were sliced using an Ultracut E microtome (Leica) with a diamond knife (Diatome). Sections were applied to Formvar-coated copper mesh grids and poststained with uranyl acetatereplacement solution for $40 \mathrm{~min}$, and $30 \mathrm{sec}$ with lead citrate. 10-nm gold particles (British Bio Cell) were applied to both sides of the grid. Single-tilt series from $-60^{\circ}$ to $+60^{\circ}$ with $1^{\circ}$ increment were acquired at $200 \mathrm{kV}$ and a 12,000-fold magnification using a JEOL electron transmission microscope (JEM 2100), Gatan Orius 1200A camera and Serial-EM software. Tomograms were generated using the IMOD package etomo. Rendering of 3-dimensional (3D) models and quantitative image analysis were performed blinded using $3 \mathrm{dmod}$. The size of synaptic ribbons and presynaptic densities (PD) was determined from the surface area of the reconstructed 3D objects. For RA-SVs the first row of vesicles around the synaptic ribbon within a distance of $80 \mathrm{~nm}$ was counted (Jung et al., 2015a). For MP-SVs the vesicles within a distance of $100 \mathrm{~nm}$ from the PD and $50 \mathrm{~nm}$ from the presynaptic plasma membrane were counted (Jung et al., 2015a). "Outlying"-SVs were counted as SVs that were located within $50 \mathrm{~nm}$ from the presynaptic plasma membrane and within the postsynaptic density area, but more than $100 \mathrm{~nm}$ from the PD. The distribution of MP-SVs and "outlying"-SVs regarding their distance to the PD and plasma membrane was analyzed by measuring the shortest (membrane-to-membrane) distance between SVs and the plasma membrane and PD. The cumulative distribution of SVs regarding their distance to the membrane and PD was calculated for each SV group. To compare the vesicle distribution statistically, the Kolmogorov-Smirnov test was applied. 


\subsection{Recordings of ABRs and DPOAEs}

Nadine Dietrich recorded ABRs and DPOAEs. For recordings of ABRs and DPOAEs mice were anesthetized with a combination of ketamine $(125 \mathrm{mg} / \mathrm{kg})$ and xylazine $(2.5 \mathrm{mg} / \mathrm{kg})$ i.p.. Their body temperature was maintained constant at $37^{\circ} \mathrm{C}$ using a heat blanket (Hugo Sachs Elektronik-Harvard Apparatus). For stimulus generation, presentation, and data acquisition, a TDT II System run by BioSig software (Tucker Davis Technologies) was used. Tone bursts (4/6/8/12/16/24/32 kHz, $10 \mathrm{~ms}$ plateau, $1 \mathrm{~ms} \cos ^{2}$ rise/fall) or clicks of $0.03 \mathrm{~ms}$ were presented at $40 \mathrm{~Hz}$ (tone bursts) or $20 \mathrm{~Hz}$ (clicks) in the free field ipsilaterally using a JBL 2402 speaker. The difference potential between vertex and mastoid subdermal needles was amplified 50,000 times, filtered $(400-4,000 \mathrm{~Hz})$ and sampled at a rate of $50 \mathrm{kHz}$ for 20 ms, 1,300 times, to obtain two mean ABR traces for each sound intensity. The hearing threshold was determined with $10 \mathrm{~dB}$ precision as the lowest stimulus intensity that evoked a reproducible response waveform in both traces by visual inspection by two independent observers. For DPOAE, continuous primary tones (frequency $f_{2=1} 1^{*} f_{1}$, intensity $I_{2}=l_{1}-10 \mathrm{~dB}$ SPL) were delivered through the MF1 speaker system (Tucker Davis Technologies) and a custom-made probe containing an MKE-2 microphone (Sennheiser). The microphone signal was amplified (DMX 6Fire, Terratec) and the DPOAE amplitude at $2^{*} f_{2}-f_{1}$ was analyzed by fast Fourier transformation using custom-written Matlab software (Mathworks). Sound pressure levels (SPL) are provided in decibel (dB) SPL root mean square (RMS) (tonal stimuli) or dB SPL peak equivalent (clicks).

\subsection{Recording of auditory nerve fiber single units}

Tanvi Butola performed extracellular field potential recordings from single units of ANFs and analyzed data. Experiments were carried out essentially as described in Jing et al. 2013. Briefly, the mice were anesthetized (urethane $1.32 \mathrm{mg} / \mathrm{kg}$, xylazine $5 \mathrm{mg} / \mathrm{kg}$, and buprenorphine $0.1 \mathrm{mg} / \mathrm{kg}$ i.p.), tracheotomized and placed in a custom-made head-holder. Their body temperature was controlled by a rectal temperature probe and maintained by heating the soundproof chamber to $26^{\circ} \mathrm{C}$ and a custom-designed heat plate. Following the partial removal of the left occipital bone and cerebellum, a high-impedance glass electrode filled with $3 \mathrm{M} \mathrm{NaCl}$ and $2 \%$ methylene blue was advanced towards the entry point of the auditory nerve into the cochlear nucleus, aiming for the SGNs. During the approach, a 50 ms noise burst search stimulus of $80 \mathrm{~dB}$ was presented at $5 \mathrm{~Hz}$ through an open field Avisoft ScanSpeak Ultrasonic Speaker (Avisoft Bioacoustics) to elicit spiking of auditory neurons. Upon detection of a sound-responsive unit, spontaneous firing rate was determined in 
silence for an initial duration of $10 \mathrm{~s}$, and if it was $<10$ or $2 \mathrm{~Hz}$, the recording duration was increased by 10 or $20 \mathrm{~s}$, respectively. Tuning curves were obtained by varying stimulus intensities and frequencies of $15 \mathrm{~ms}$ tone bursts, to estimate the best threshold of hearing and the characteristic frequency with a $2 \mathrm{~dB}$ and 1/32 octave precision, respectively. Peristimulus time histograms (PSTHs) to 50 ms tone burst stimulation or paired stimuli (forward masking) were obtained at the characteristic/best frequency at sound intensities at which spike rates were mostly saturated: $30 \mathrm{~dB}$ above threshold.

SGNs were differentiated from cochlear nucleus neurons by (i) the electrode position $(>1,200 \mu \mathrm{m}$ below the surface of the cochlear nucleus), (ii) their primary-like PSTHs to suprathreshold $50 \mathrm{~ms}$ tone bursts presented at the characteristic/best frequency and (iii) their irregular firing pattern, as confirmed by a coefficient of variance (CV) of inter-spike interval of steady state firing response, $>0.5$. Recordings were performed using TDT system III hardware and an ELC-03XS amplifier (NPI electronics), offline analysis using waveformbased spike detection using custom-written MATLAB software. Statistical tests employed for data analysis accompany respective figure legends.

\subsection{Data analysis and statistical tests}

Data analysis and statistical tests on electrophysiology, Ca ${ }^{2+}$-imaging, STED microscopy, and electron tomography data were done in Igor Pro software (Wavemetrics, Lake Oswego, USA). Normality of data was assessed with the Jarque-Bera test. The F-test was used to assess equality of variance in normally distributed data sets. The unpaired, two-tailed Wilcoxon rank test (Mann-Whitney test) was used to compare non-normal or data with unequal variances. Else, the unpaired, two-tailed Student's t-test was used. To assess the equality of sample variances of the $\Delta \mathrm{F} / \mathrm{F}_{0}$ estimates for synaptic $\mathrm{Ca}^{2+}$-signals, a modified Levene's test, using median instead of mean for improved robustness under non-normality was used. Data analysis for systems physiology experiments was done in MATLAB software. For statistical analysis of ABR and DPOAE data, a one-way ANOVA test and, for multiple comparisons, post-hoc Tukey's test were used in Graphpad Prism. Statistical significance is indicated by ${ }^{*}$ for $p<0.05,{ }^{* *}$ for $p<0.01$, and ${ }^{* * *}$ for $p<0.001$. Data are presented as mean \pm standard error of the mean (SEM), unless otherwise specified. Graphs were done with Igor Pro software (Wavemetrics, Lake Oswego, USA) and figures were assembled in Adobe Illustrator software (Adobe Systems Software Ireland Ltd.). 


\section{Chapter 1}

"RIM-binding protein 2 promotes the number of voltage-gated Cav1.3 $\mathrm{Ca}^{2+}$-channels at hair cell active zones and contributes to fast synaptic vesicle recruitment" 


\subsection{Introduction}

In order to indefatigably encode stimulating sounds with sub-millisecond precision, ribbon synapses of IHCs are specialized for high rates of synchronous exocytosis. As discussed in the General Introduction, a sophisticated, unconventional molecular machinery of cytomatrix proteins realizes this impressive performance. They regulate the number of synaptic voltage-gated Cav1.3 $\mathrm{Ca}^{2+}$-channels, tightly couple SVs to $\mathrm{Ca}^{2+}$-channels and provide fast replenishment of fusion-competent SVs for sustained exocytosis. Besides the CAZ proteins RIM (Coppola et al., 2001; Gebhart et al., 2010; Jung et al., 2015a; Kaeser et al., 2011; Kiyonaka et al., 2007), and bassoon (Davydova et al., 2014; Frank et al., 2010; Khimich et al., 2005), both playing a critical role in regulating the abundance of $\mathrm{Ca}^{2+}$ channels at IHC AZs, lately the focus has turned to RIM-BPs (Figure 5, green). RIM-BPs play a central role for AZ function by acting as molecular hub between voltage-gated $\mathrm{Ca}^{2+}$ channels and vesicular release sites (Hibino et al. 2002; Acuna et al. 2015; M. Müller, Genç, and Davis 2015; Grauel et al. 2016).

Vertebrates express three RIM-BP isoforms - RIM-BP1 and 2 are primarily found in the brain, whereas RIM-BP3 is expressed outside the brain (Mittelstaedt and Schoch, 2007). All RIM-BPs contain an N-terminal and two C-terminal Src homology domains (SH3) and one Fibronectin type II domain (Figure 11). The N-terminal SH3 domain interacts with the proline-rich PXXP motif of bassoon (Davydova et al., 2014). The two C-terminal SH3 domains interact with RIMs and the $\alpha_{1}$ subunits of voltage-gated P/Q-, N-, and L-type $\mathrm{Ca}^{2+}$ channels (Hibino et al., 2002; Kaeser et al., 2011; Wang et al., 2000).

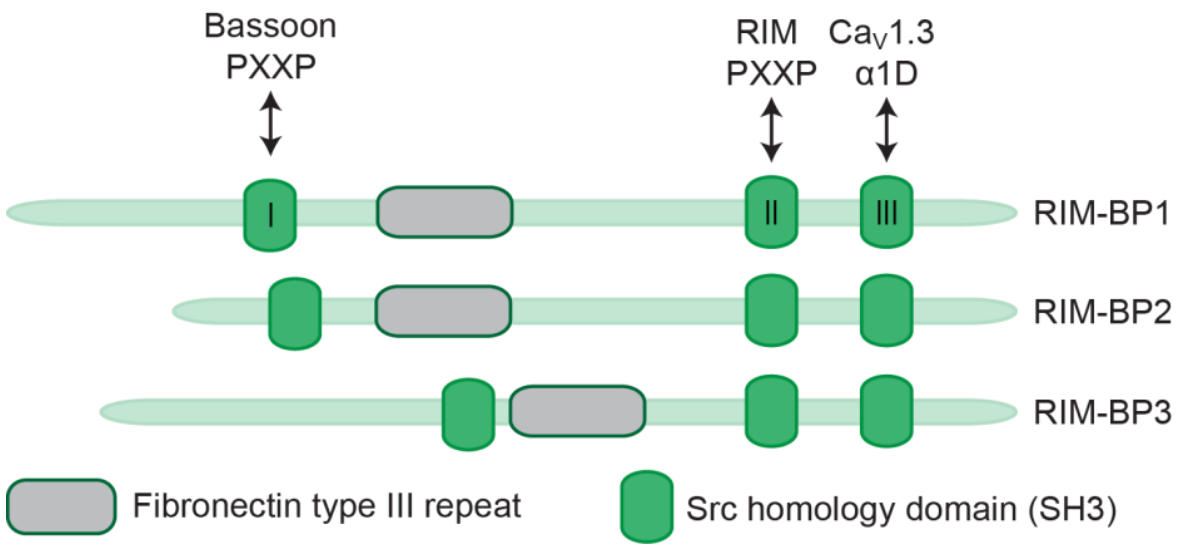

Figure 11: Multidomain structure and interaction sites of RIM-binding proteins All three RIM-BP isoforms contain an N-terminal and two C-terminal Src homology domains (SH3) (green) and one Fibronectin type II domain (gray). The N-terminal SH3 domain (I) interacts with proline-rich PXXP motif of bassoon; the two C-terminal SH3 domains (II and III) interact with proline-rich PXXP motif of RIMs and the $\alpha_{1}$ subunit of voltage-gated P-/Q-, $\mathrm{N}-$, and L-type $\mathrm{Ca}^{2+}$-channels, respectively. 
These various protein interactions potentially enable RIM-BPs to regulate synaptic $\mathrm{Ca}^{2+}$ channels and bring SVs into close vicinity to $\mathrm{Ca}^{2+}$-channels and their release sites and suggest RIM-BPs as a central node at the AZ function. Studies in mammalian hippocampal synapses and the calyx of Held however reported only moderate effects of RIM-BP disruption on synapse structure and functionality (Acuna et al. 2015; Grauel et al. 2016). In hippocampal synapses, RIM-BP2 was found to fine-tune the clustering of P/Q-type $\mathrm{Ca}^{2+}$ channels at the AZ (Grauel et al., 2016): More distant positioning of the P/Q-type $\mathrm{Ca}^{2+}$ channels to vesicular release sites upon disruption of RIM-BP2 affected the $\mathrm{Ca}^{2+}$ nanodomains. Consequently, the SV release sensitivity to the slow $\mathrm{Ca}^{2+}$-buffer EGTA increased, and the initial release probability of SVs decreased (Grauel et al., 2016). Additional disruption of RIM-BP1 did not lead to further significant effects on synapse structure or function (Grauel et al., 2016). Studies from the calyx of Held reported that double knockout of $R I M-B P 1$ and $R I M-B P 2$ impaired the fidelity, reliability, and magnitude of neurotransmitter release, but not physiological $\mathrm{Ca}^{2+}$-channel properties (Acuna et al. 2015). Based on an increased sensitivity of SV release to presence of the slow $\mathrm{Ca}^{2+}$-buffer EGTA, an increased coupling distance between $\mathrm{Ca}^{2+}$-channels and SVs was proposed. Therefore, RIM-BPs might have a selective role in coupling $\mathrm{Ca}^{2+}$-influx to SV-exocytosis during AP-driven transmitter release (Acuna et al. 2015). Compared to the relatively mild phenotype in mammalian synapses, the RIM-BP disruption phenotype in Drosophila melanogaster was more substantial. In Drosophila melanogaster, the RIM-BP ortholog DRBP was found to be essential for both the structural and functional integrity of the $A Z$ of NMJs. In the absence of DRBP the presynaptic cytomatrix was disorganized, with no regularly shaped T-bars visible, impaired $\mathrm{Ca}^{2+}$-channel clustering and $\mathrm{Ca}^{2+}$-influx, and looser $\mathrm{Ca}^{2+}$-influx-exocytosis coupling, together resulting in a decreased SV release probability. Further, RRP recovery during paired-pulse stimulation was drastically reduced (Liu et al., 2011; Müller et al., 2015).

At IHC AZs, RIM-BPs might turn out to be functionally especially relevant because of (1) the apparent lack of some CAZ proteins at IHC ribbon synapses (Vogl et al., 2015) that are essential at conventional synapses, (2) the direct interaction of RIM-BPs with bassoon, RIM2, and the pore-forming $\alpha_{1 D}$ subunit of L-type voltage-gated Cav1.3 $\mathrm{Ca}^{2+}$-channels (Hibino et al. 2002; Liu et al. 2011; M. Müller, Genç, and Davis 2015; Acuna et al. 2015; Davydova et al. 2014) and (3) a similar synapse architecture of IHC ribbon synapses to Drosophila melanogaster NMJs with its prominent T-bar dense projection (Wichmann and Sigrist, 2010). Whether RIM-BPs are expressed at IHC ribbon synapses and which functional role they might take as part of the unconventional synaptic machinery (Rutherford and Pangršič, 2012) remained to be investigated. Given their role at other synapses, RIM$\mathrm{BPs}$ are promising candidates to regulate the abundance of presynaptic $\mathrm{Ca}^{2+}$-channels and 
to contribute to the $\mathrm{Ca}^{2+}$ nanodomain-like control of SV exocytosis at the IHC AZ. In this part of my PhD work, multidisciplinary state-of-the-art techniques and a constitutive RIM$B P 2$ knockout mouse model were employed to characterize the function of RIM-BP2 in synaptic transmission in IHCs of the mouse cochlea.

\subsection{Results}

\section{RIM-BP2 forms (double-) stripe shaped clusters at IHC AZs}

First, the expression and localization of RIM-BP2 at IHC AZs was investigated using immunohistochemistry and 2-color confocal microscopy of mature mouse IHCs (Figure 12). RIM-BP2 immunofluorescence (green) co-localized with presynaptic ribbons of IHC AZs, marked by CtBP2/RIBEYE (magenta). Specificity of the immunolabeling was confirmed by the absence of RIM-BP2 immunofluorescence in simultaneously processed littermate RIM$B P 2^{-1-}$ IHCs (Figure 12A). In addition, some RIM-BP2 immunofluorescence spots were observed that did not co-localize with IHC ribbons. Most likely, they reflect expression of RIM-BP2 at conventional presynaptic terminals of efferent ANFs of lateral olivocochlear neurons projecting onto the postsynaptic boutons of the afferent SGNs adjacent to the base of the IHCs (connectivity illustrated in Figure 12B). Triple antibody staining for RIM-BP2, CtBP2/RIBEYE, and synapsin1/2, a marker for presynaptic terminals of efferent lateral olivocochlear neurons (Safieddine and Wenthold, 1999) (Figure 12B) confirmed this hypothesis. As RIM-BP2 immunofluorescence co-localized with either CtBP2/RIBEYE or synapsin1/2, but was not visible outside the marked presynaptic regions, RIM-BP2 likely specifically localizes to AZs of both IHCs and terminals of efferent lateral olivocochlear neurons.

In addition, the nanoscale organization of RIM-BP2 at IHC AZs was determined using 2D 2-color STED microscopy. A mostly stripe-like and double-stripe-like cluster shape of RIMBP2 immunofluorescence (green) was observed at the base of synaptic ribbons (CtBP2/RIBEYE, magenta), which could not be resolved by confocal microscopy (Figure 12C). The clusters appear in a similar stripe-like pattern as other IHC cytomatrix proteins like bassoon and RIM2, which co-align with the presynaptic $\mathrm{Cav}_{\mathrm{v}} 1.3 \mathrm{Ca}^{2+}$-channel clusters (Frank et al., 2010; Jung et al., 2015a). So far, a double-stripe like cluster shape was only described for Cav1.3 $\mathrm{Ca}^{2+}$-channels (Frank et al., 2010; Wong et al., 2014), suggesting a functional link between Cav1.3 $\mathrm{Ca}^{2+}$-channels and RIM-BP2 as central part of the presynaptic CAZ. Co-immunostaining of RIM-BP2 together with Cav1.3 $\mathrm{Ca}^{2+}$-channels was not possible, since the antibodies were available only from the same species (see Methods and Materials). 
A RIM-BP2 CtBP2/RIBEYE confocal

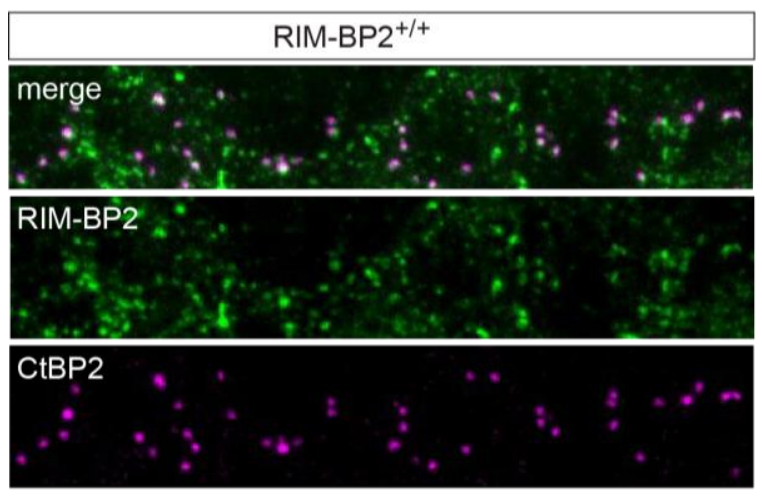
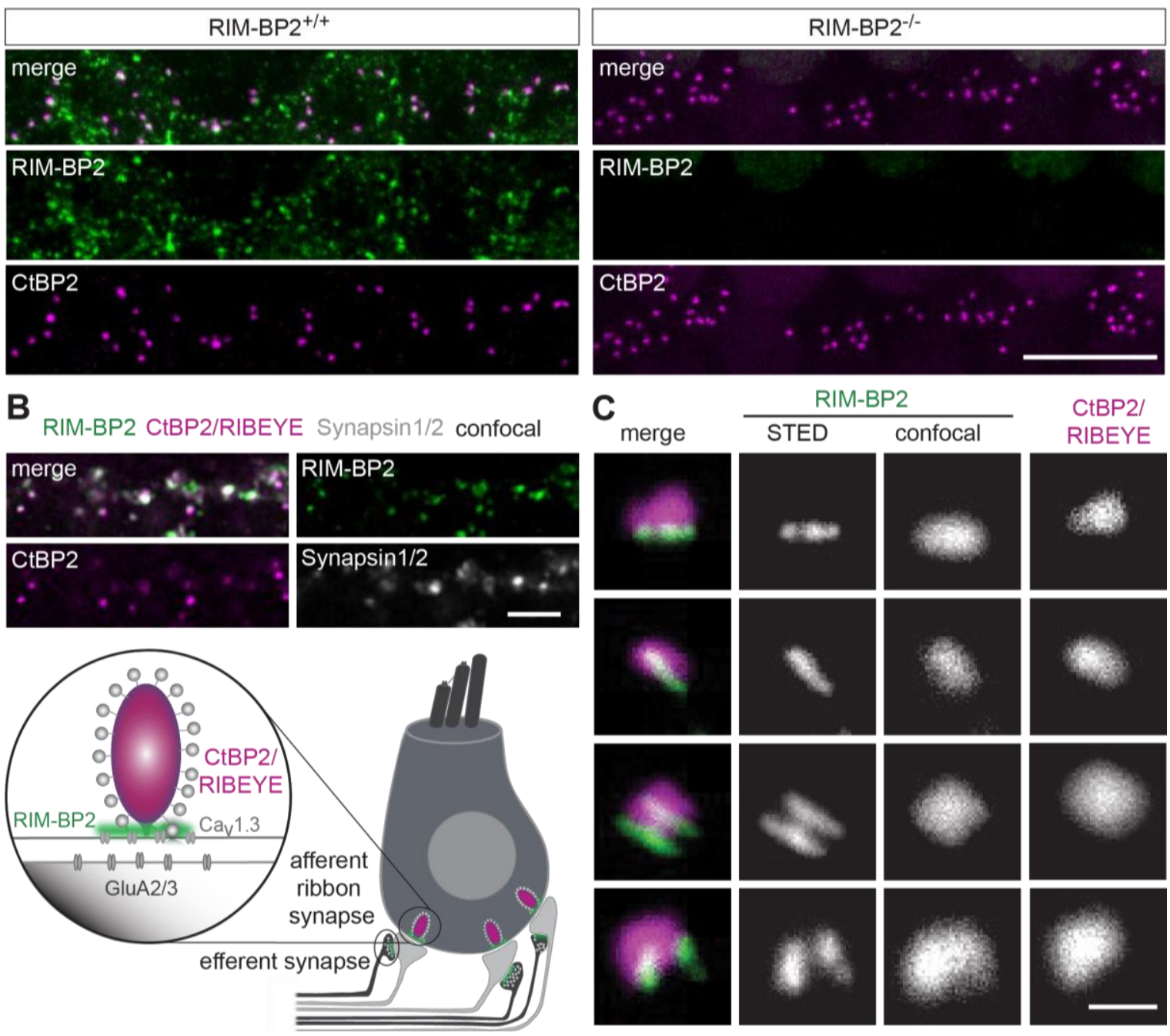

C

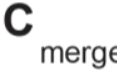

RIM-BP2
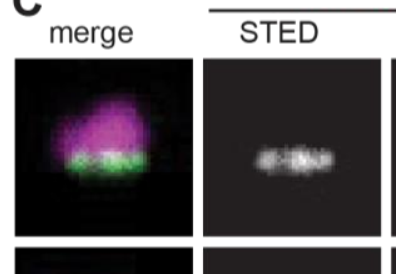

confocal

CtBP2/
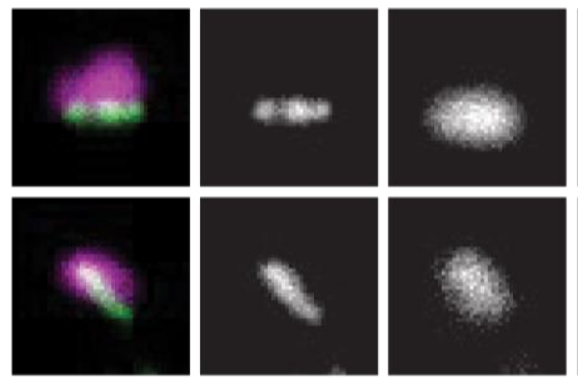

RIBEYE
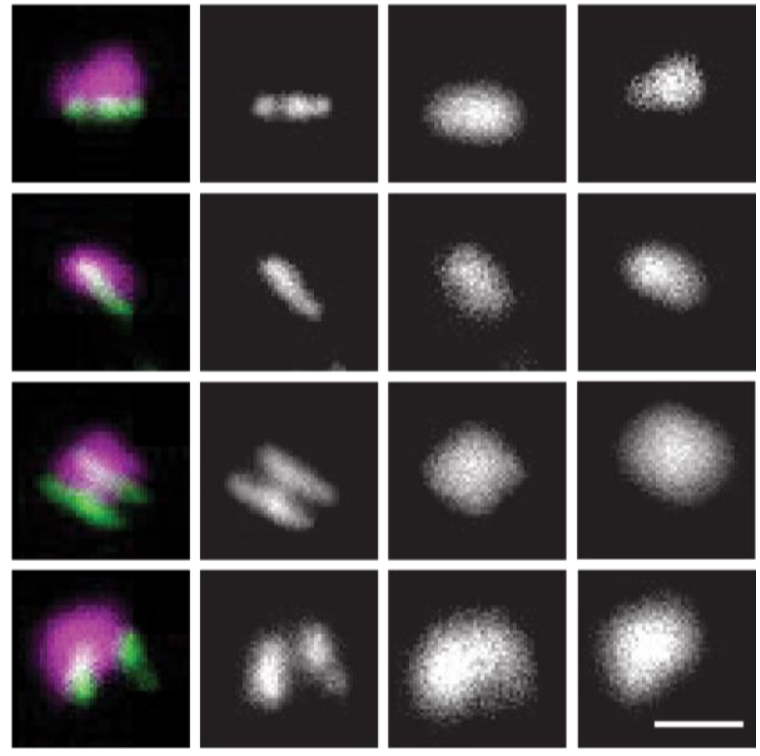

Figure 12: RIM-BP2 forms stripe like clusters at the base of the IHC synaptic ribbon

[A] Expression analysis of RIM-BP2 in IHCs: Maximum projections of confocal stacks after immunohistochemistry of whole mount explants of apical organs of Corti of p21 mice. RIMBP2 immunofluorescence (green) co-localizes with presynaptic ribbons (CtBP2/RIBEYE, magenta) in $R I M-B P 2^{+/+}$IHCs and is absent in parallel processed littermate $R I M-B P 2^{-/}$ IHCs, indicating specificity of the immunolabeling. Additional RIM-BP2 fluorescence in RIM$\mathrm{BP}^{+++} \mathrm{IHCs}$ most likely localizes to presynaptic efferent terminals (illustrated in B). Scale bar: $10 \mu \mathrm{m}$. [B] Top: Confocal stacks (maximum projections) after triple-immunostaining of whole-mount explants of apical organs of Corti of p21 mice. RIM-BP2 immunofluorescence (green) co-localizes with either presynaptic ribbons (CtBP2/RIBEYE, magenta) or synapsin1/2 (gray), a marker of presynaptic terminals of efferent lateral olivocochlear neurons. Scale bar $5 \mu \mathrm{m}$. Bottom: Illustration of a mature murine IHC: Afferent type I SGNs innervate ribbon synapses at the IHC base. Efferent synapses of the lateral olivocochlear neurons form projections onto type I SGN synapses nearby the IHC. Location of presynaptic ribbons (CtBP2/RIBEYE) is highlighted in magenta. RIM-BP2 (green) forms clusters at the $P D$ at the base of the synaptic ribbon and at the AZs of efferent presynaptic terminals. [C] Nanoscale organization of RIM-BP2: 2-color STED microscopy of individual AZs from whole mount explants of apical organs of Corti of p21 mice after immunohistochemistry resolved a mostly stripe or double-stripe-like expression pattern of RIM-BP2 (green) at the ribbon (CtBP2/RIBEYE, magenta). Simultaneously acquired confocal images could not resolve single or double stripes of RIM-BP2. Single XY-sections, Scale bar: $500 \mathrm{~nm}$. Panels [A-C] adapted from Krinner et al., 2017. 


\section{RIM-BP2 promotes presynaptic $\mathrm{Ca}^{2+-i n f l u x ~ i n ~ I H C s ~}$}

RIM-BPs interact with the pore-forming and voltage-sensitive $\alpha_{1 \mathrm{D}}$ subunit of $\mathrm{Cav} 1.3 \mathrm{Ca}^{2+}$ channels (Coppola et al., 2001; Hibino et al., 2002) and clusters in a similar stripe-shaped fashion as Cav1.3 $\mathrm{Ca}^{2+}$-channels (Figure 12C). Therefore, the effect of RIM-BP2 disruption on presynaptic $\mathrm{Ca}^{2+}$-influx and $\mathrm{Ca}^{2+}$-channel gating was studied, using the perforated patchclamp technique to record voltage-gated $\mathrm{Ca}^{2+}$-influx in $\mathrm{RIM-BP2^{-/ }}$ IHCs.

The $\mathrm{Ca}^{2+}$ IV revealed a significant (20\%) reduction of the maximal whole-cell $I_{C a}$ amplitude in $R I M-B P 2^{-/}$IHCs (Figure 13A). Further, the $\mathrm{Ca}^{2+}$-channel gating, including the $\mathrm{V}_{\text {half }}$ and the slope factor of fractional activation (k), (Figure 13B) as well as the kinetics of $I_{C a}$ inactivation ( $\tau_{\text {inactivation}}$, Figure $13 \mathrm{C}$ ) were analyzed, each of which was unaltered in RIM-BP2 - IHCs (Table 3). The channel open probability and single channel current were not tested in $R I M-B P 2^{-1}$ IHCs and thus cannot be excluded as a cause of the reduced $I_{C a}$ amplitude. Since no changes in the $\mathrm{Ca}^{2+}$-channel gating and kinetics were observed as consequence of $R I M-B P 2$ disruption, these results favor the hypothesis of reduced overall $\mathrm{Ca}^{2+}$-channel numbers due to loss of RIM-BP2.

Table 3: Summary of $\mathrm{Ca}^{2+}$-current $\left(\mathrm{I}_{\mathrm{Ca}}\right)$ data from perforated patch-clamp recordings

\begin{tabular}{|c||c|c|c|c|}
\hline & Amplitude (pA) & $\mathbf{V}_{\text {half }}(\mathbf{m V})$ & Slope factor $\mathbf{k}$ & $\tau_{\text {inactivation }}(\mathbf{m s})$ \\
\hline \hline $\boldsymbol{R I M - B P 2} 2^{+/+}$ & $-157 \pm 6(\mathrm{n}=17)$ & $-29.9 \pm 0.9(\mathrm{n}=17)$ & $6.9 \pm 0.1(\mathrm{n}=17)$ & $90 \pm 11(\mathrm{n}=15)$ \\
\hline $\boldsymbol{R} I M-\mathbf{B P 2}^{-/}$ & $-126 \pm 9(\mathrm{n}=16)$ & $-30 . \pm 1.5(\mathrm{n}=16)$ & $6.7 \pm 0.2(\mathrm{n}=16)$ & $98 \pm 13(\mathrm{n}=11)$ \\
\hline $\boldsymbol{p}$-value & $\begin{array}{c}0.02 \\
\text { Wilcoxon rank } \\
\text { test }\end{array}$ & $\begin{array}{c}0.9 \\
\text { Student's t-test }\end{array}$ & $\begin{array}{c}\text { Wilcoxon rank } \\
\text { test }\end{array}$ & $\begin{array}{c}\text { Wilcoxon rank } \\
\text { test }\end{array}$ \\
\hline
\end{tabular}

Summary of $\mathrm{IHC}$ average $\mathrm{Ca}^{2+}$-current $\left(I_{C a}\right)$ data from perforated patch-clamp recordings from $R I M-B P 2^{+/+}$and RIM-BP2-- IHCs (Figure 13). Whole cell $I_{C a}$ was analyzed regarding amplitude, $\mathrm{V}_{\text {half, }} \mathrm{Ca}^{2+}$-channel gating (slope factor $\mathrm{k}$ representing voltage sensitivity of $\mathrm{Ca}^{2+}$ influx), and kinetics of $I_{C a}$ inactivation ( $\tau_{\text {inactivation }}$ ). Data represent IHC grand averages, mean \pm SEM; $n=$ number of IHCs; $p$-values and statistical test are depicted for each dataset, significance level: n.s. $p \geq 0.05,{ }^{*} p<0.05,{ }^{* *} p<0.01,{ }^{* \star} p<0.001$. Table adapted from Krinner et al., 2017. 


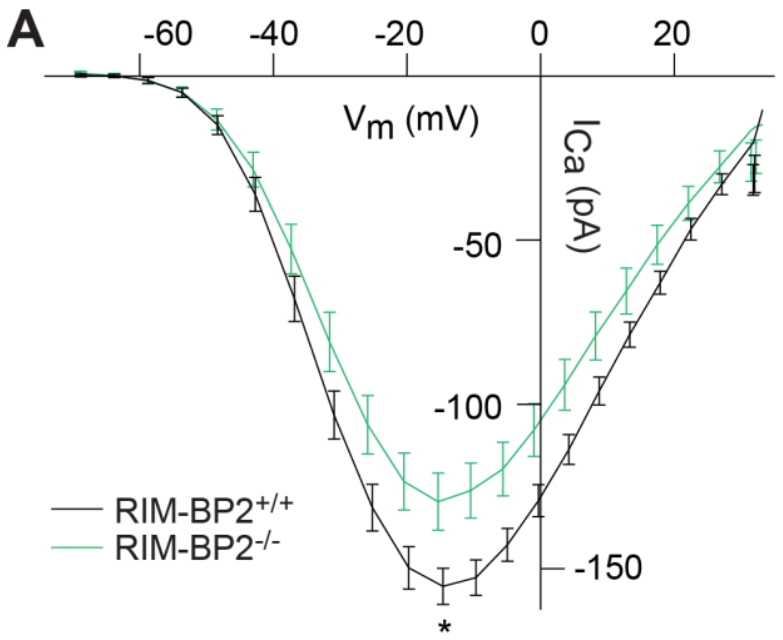

\section{Figure 13: RIM-BP2 promotes voltage-dependent $\mathrm{Ca}^{2+}$-influx in IHCs}

[A] $\mathrm{Ca}^{2+}$ IV of RIM-BP2 deficient ( $R I M-$ $B P 2^{--}, \mathrm{n}=16$, green) and control (RIM$B P 2^{+/+}, \mathrm{n}=17$, black) IHCs. $\mathrm{Ca}^{2+}$ current $\left(I_{c a}\right)$ amplitude was significantly reduced in RIM-BP2 deficient IHCs.

[B] Fractional activation curves of the whole-cell $\mathrm{Ca}^{2+}$-current: A Boltzmann function was fitted to the normalized conductance curve calculated from the $\mathrm{Ca}^{2+}$ IV $[\mathrm{A}]$. Average fit data (dashed traces) are displayed for both genotypes ( $R I M-B P 2^{-/}, \mathrm{n}=16$, green, and $R I M-B P 2^{+/+}, \mathrm{n}=17$, black). Dashed lines (gray) indicate $V_{\text {half }}$ of the wholecell $\mathrm{Ca}^{2+}$-current.

[C] $\mathrm{Ca}^{2+}$-current $\left(I_{C a}\right)$ during 200-ms step depolarization to $-14 \mathrm{mV}$ of RIMBP2 deficient (RIM-BP2 ${ }^{-/}, \quad \mathrm{n}=11$, green) and control $\left(R I M-B P 2^{+/+}, \mathrm{n}=15\right.$, black) IHCs. Direct comparison of the $\mathrm{Ca}^{2+}$-currents through scaling of the $\mathrm{Ca}^{2+}$-currents showed no difference in the $\mathrm{Ca}^{2+}$-current kinetics.

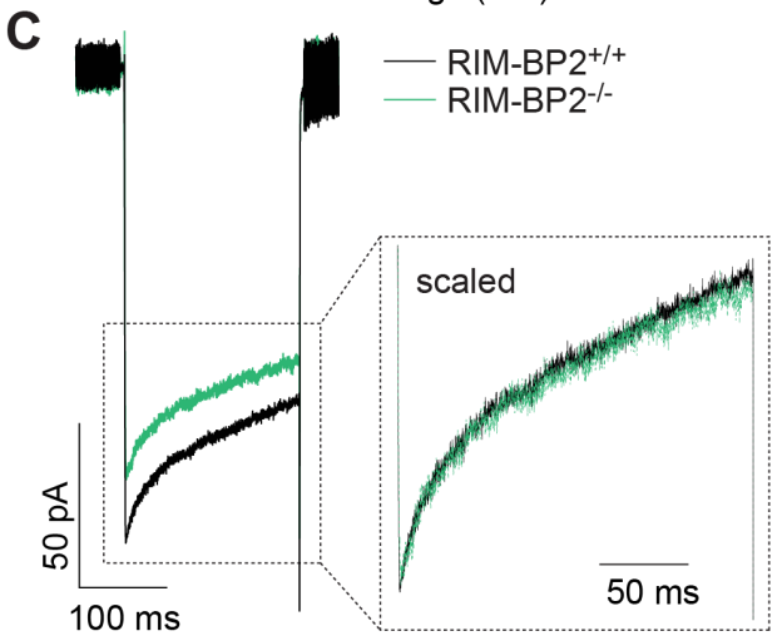

[A-C] Data information: Data represent IHC grand averages, mean \pm SEM. Mean \pm SEM and statistical tests and $p$-values are displayed in Table 3. Significance level: n.s. $p \geq 0.05,{ }^{*} p<0.05,{ }^{* *} p<0.01,{ }^{* * *} p<0.001$; $\mathrm{n}=$ number of IHCs; age of mice: $\mathrm{p} 14-$ p16. Panels $[\mathrm{A}-\mathrm{C}]$ adapted from Krinner et al., 2017.

Since whole-cell patch-clamp recordings represent the summed $\mathrm{Ca}^{2+}$-current across the whole IHC's plasma membrane, including extra-synaptic $\mathrm{Ca}^{2+}$-influx, the $\mathrm{Ca}^{2+}$-influx at individual AZs was investigated as a next step, using laser scanning confocal $\mathrm{Ca}^{2+}$-imaging (Francis et al., 2011; Frank et al., 2009; Ohn et al., 2016). 
A Ruptured whole-cell patch-clamp
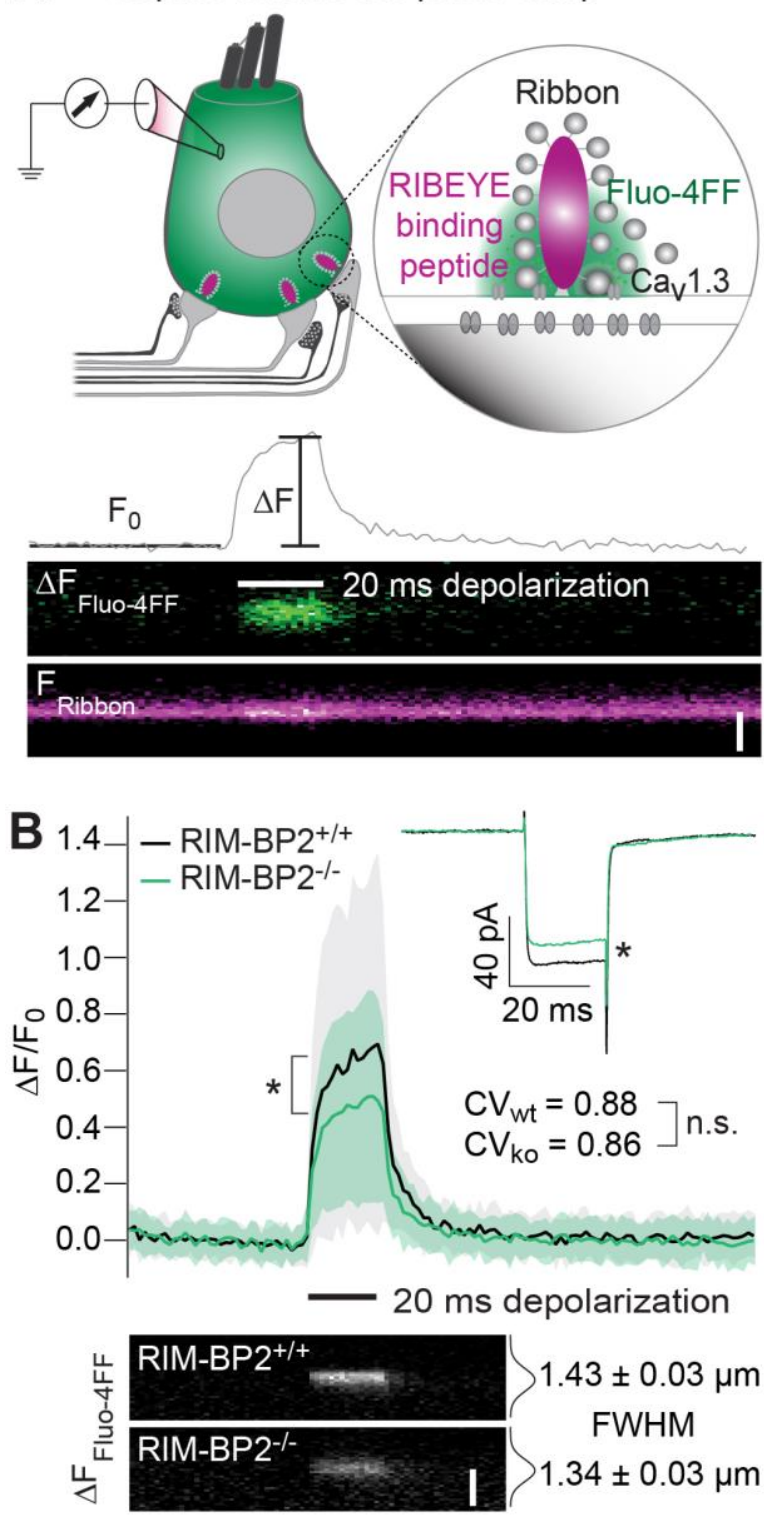

Figure 14: RIM-BP2 promotes the abundance of synaptic $\mathrm{Ca}^{2+}$. channels in IHCs

[A] Top: schematic representation of an IHC loaded with fluorescently labeled RIBEYE-binding peptide $(10 \mu \mathrm{M})$ to visualize ribbons at the $A Z$, and a low affinity $\mathrm{Ca}^{2+}$ indicator Fluo-4FF (400 $\mu \mathrm{M})$ to monitor $\mathrm{Ca}^{2+}$ influx. Bottom: Confocal line scan across an IHC AZ showing ribbon fluorescence ( $\left.F_{\text {ribbon }}\right)$ and Fluo-4FF fluorescence change $\left(\Delta \mathrm{F}_{\text {Fluo-4FF }}\right)$ during $20 \mathrm{~ms}$ depolarization (white bar). Temporal profile of Fluo4FF fluorescence from the ribbon center illustrates the calculated $\Delta \mathrm{F}$, which was normalized to its baseline fluorescence $F_{0}$ hence $\Delta F / F_{0}$. Scale bar: $2 \mu \mathrm{m}$.

[B] Top: Average $\triangle \mathrm{F} / \mathrm{F}_{0}$ for $R I M-B P 2^{+/+}$ $\left(\mathrm{n}=41, \mathrm{~N}=14\right.$, black line) and $R I M-B P 2^{-1}$ $(\mathrm{n}=42, N=13$, green line) AZs during IHC depolarizations (black bar). $\Delta \mathrm{F} / \mathrm{F}_{0}$ (grand averages, mean \pm SD as shaded area) and its corresponding $I_{C a}$ (grand average) were significantly reduced in $R I M-B P 2^{--}$IHCs (Wilcoxon rank test, both $p=0.01$ ). The CV (mean) was comparable within genotypes (modified Levene's test). Bottom: Representative $\mathrm{Ca}^{2+}$-signals (same intensity-scale) of $R I M-B P 2^{+/+}$and RIM-BP2 ${ }^{-/} \quad$ AZs showing comparable spatial spread of synaptic $\mathrm{Ca}^{2+}$-influx estimated by a Gaussian fit (Student's t-test, $p=0.5$; mean \pm SEM). Scale bar: $2 \mu \mathrm{m}$. Data information: Significance levels: n.s. $p \geq$ $0.05,{ }^{*} p<0.05,{ }^{* *} p<0.01,{ }^{* * *} p<0.001$; $\mathrm{n}=$ number of $\mathrm{AZs}, \mathrm{N}=$ number of $\mathrm{IHCs}$; age of mice: p14-16. Panels [A-B] adapted from Krinner et al., 2017..

The ruptured patch-clamp technique was performed to load IHCs with the low affinity $\mathrm{Ca}^{2+}$ indicator Fluo-4FF ( $\left.\mathrm{K}_{\mathrm{D}} 10 \mu \mathrm{M}\right)$ to monitor $\mathrm{Ca}^{2+}$-signals, and in addition with a fluorescently conjugated dimeric RIBEYE-binding peptide (Francis et al., 2011) to visualize the location of synaptic ribbons. During $\mathrm{IHC}$ depolarization (20 ms to $-7 \mathrm{mV}$ ) line scans were performed across the center of fluorescently labeled AZs to measure synaptic $\mathrm{Ca}^{2+}$-indicator fluorescence (Figure 14A). 
A significant decrease in the average synaptic Fluo-4FF fluorescence change $\left(\Delta F / F_{0}\right)$ was observed at $R I M-B P 2^{--}$AZs ( $p=0.01$, Figure 14B). Under the chosen experimental conditions ([C $\left.\mathrm{Ca}^{2+}\right]_{\mathrm{e}}: 2 \mathrm{mM}, \mathrm{Ca}^{2+}$-buffering: $1 \mathrm{mM}$ EGTA) Fluo-4FF fluorescence changes $\left(\Delta \mathrm{F} / \mathrm{F}_{0}\right)$ are expected to be a good proxy for the synaptic $\mathrm{Ca}^{2+}$-influx (Frank et al., 2009). The greater reduction in synaptic $\mathrm{Ca}^{2+}$-influx $(41 \%)$ compared to that of the whole-cell $\mathrm{Ca}^{2+}-$ current (20\% reduction, Figure $13 \mathrm{~A}, p=0.01)$, indicates that RIM-BP2 preferentially regulates the number of synaptic $\mathrm{Ca}^{2+}$-channels and that RIM-BP2 deficient AZs may tether synaptic $\mathrm{Ca}^{2+}$-channels less efficiently.

Within IHCs AZs, $\Delta F / F_{0}$ estimates vary greatly (Figure 14B), which are representative of the commonly observed heterogeneity of ribbon synapses regarding their $\mathrm{AZ}$ size and $\mathrm{Ca}^{2+}$ channel number and gating (Frank et al., 2009; Meyer et al., 2009; Ohn et al., 2016; Wong et al., 2014). Synapse heterogeneity was assessed by calculating the CV of the line scan $\Delta F / F_{0}$ signals between 15 and 20 ms after depolarization onset and was comparable between the genotypes ( $R I M-B P 2^{+/+} \mathrm{AZs} C V=0.88, R I M-B P 2^{-/} \mathrm{CV}=0.86$, Figure $\left.14 \mathrm{~B}\right)$. This indicates that even though the overall synaptic $\mathrm{Ca}^{2+}$-influx amplitude was reduced, synapse heterogeneity was unchanged. The spatial extent of synaptic $\mathrm{Ca}^{2+}$-signals was estimated by fitting a $1 \mathrm{D}$ Gaussian function to the maximal $\Delta \mathrm{F}_{\text {Fluo-4FF }}$ amplitudes of the line scan $15 \mathrm{~ms}$ after depolarization onset. Compared to $R I M-B P 2^{+/+} \mathrm{AZs}$, the FWHM of $R I M-B P 2^{-/} \mathrm{AZs}$ tended to be reduced without reaching statistical significance $\left(R I M-B P 2^{+/+} \mathrm{FWHM}=1.43 \pm\right.$ $0.03 \mu \mathrm{m}, R I M-B P 2^{-/} \mathrm{FWHM}=1.34 \pm 0.03 \mu \mathrm{m}, p=0.5$, Figure 14B).

However, as confocal $\mathrm{Ca}^{2+}$-imaging under low intracellular $\mathrm{Ca}^{2+}$-buffering conditions (1 $\mathrm{mM}$ EGTA) does not provide insight into the nanoscale organization of $\mathrm{Ca}^{2+}$-channels at the $A Z$, super-resolution STED microscopy of Cav1.3 immunofluorescence was used next. Immunohistochemistry for Cav1.3 $\mathrm{Ca}^{2+}$-channels (Figure 15A: green) and the presynaptic ribbon (CtBP2/RIBEYE, Figure 15A: magenta) followed by 2-color STED microscopy was performed. Different apparent shapes of Cav1.3 immunofluorescence (likely reflecting synaptic Cav1.3 $\mathrm{Ca}^{2+}$-channel clusters) at ribbon-occupied AZs were first subjectively classified (Figure 15A) and then analyzed in a semi-quantitative way (Figure 15C, D). 


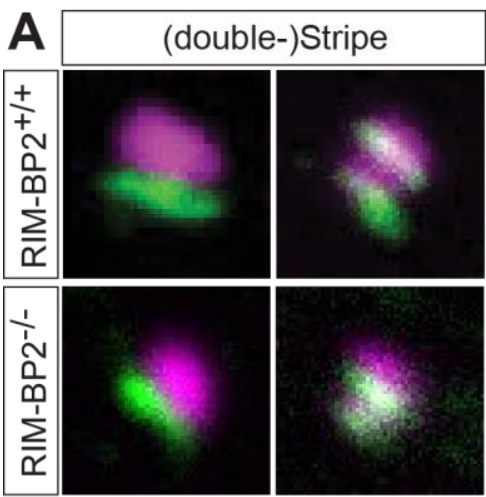

$\mathrm{Ca}_{\mathrm{V}} 1.3$ STED CtBP2 STED
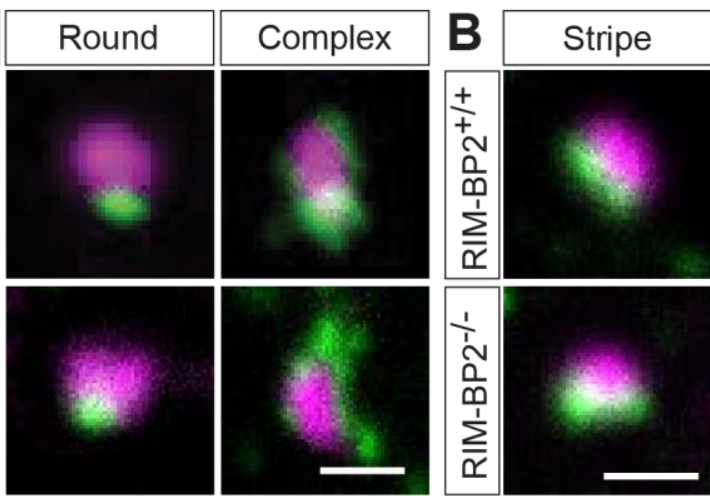

Bassoon STED CtBP2 STED

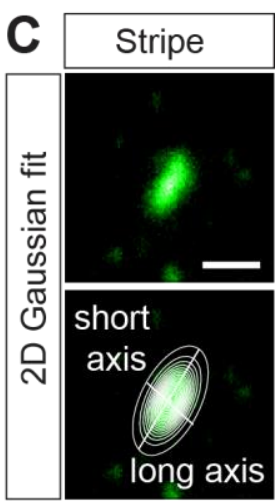

$\mathrm{Ca}_{\mathrm{V}} 1.3$ example STED
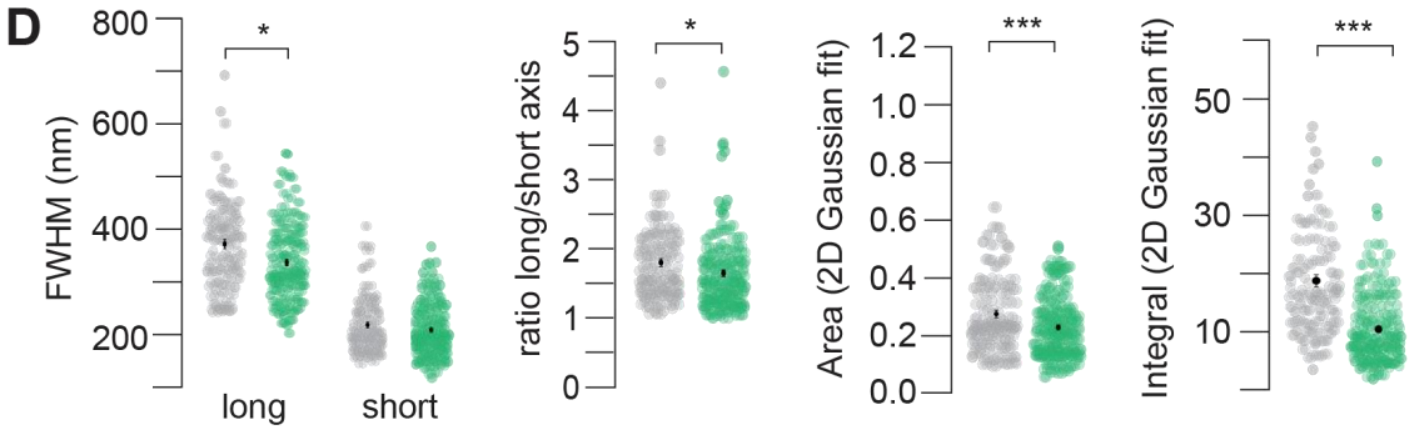

$\mathrm{Ca}_{\mathrm{v}} 1.3$

RIM-BP2 $2^{+/+}$

RIM-BP2 ${ }^{-/-}$

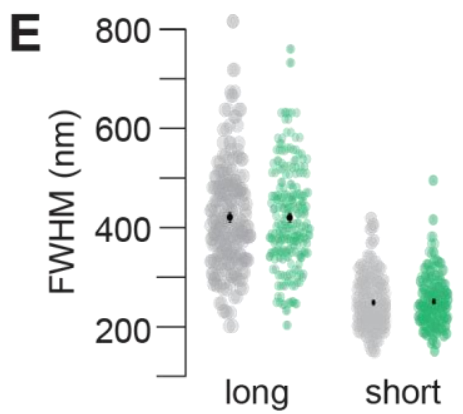

Bassoon

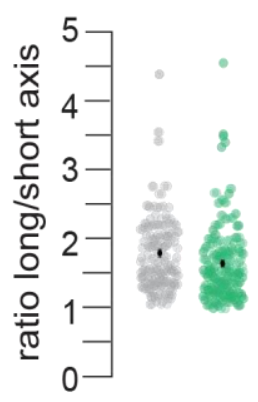

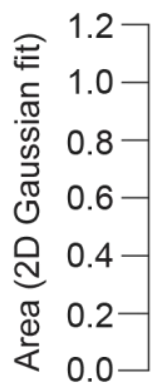

RIM-BP $2^{+/+}$

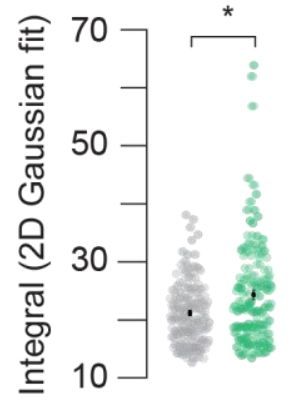

RIM-BP2 $2^{-/-}$

Figure 15: RIM-BP2 promotes $\mathrm{Ca}^{2+}$-channel abundance at IHC ribbon synapse AZs

[A,B] XY-sections of individual AZs acquired with high-resolution 2D 2-color STED microscopy. Whole mount explants of apical $R I M-B P 2^{+/+}$and $R I M-B P 2^{--}$organs of Corti (age: p21-p23) were immunolabeled for CtBP2/RIBEYE (magenta) and Cav1.3 (green, $[A]$ ) or bassoon (green, [B]). Scale bars: $500 \mathrm{~nm}$. [A] Cav1.3 clusters were subjectively classified in "(double-) stripe-like", "round" and "complex" shapes. [C] 2D Gaussian fit functions were fitted (illustrated at bottom) to each individual STED image of stripe-shaped Cav1.3 (example at top) and bassoon clusters to approximate the cluster dimensions. Fit amplitudes and FWHM of the long and short axis (illustrated) of the 2D Gaussian fit function were used for quantitative analysis in D, E. Scale bar: $500 \mathrm{~nm}$. [D, E] Results of quantitative analysis of Cav1.3 [D] and bassoon [E] cluster STED images as described in [C]. Data of FWHM long and short axis, the ratio of long over short axis, and cluster area and integral are displayed as individual data points $\left(R I M-B P 2^{+/+}\right.$in gray, $R I M-B P 2^{-/}$in green) and grand averages mean \pm SEM (black). [D] $R I M-B P 2^{+/+} \mathrm{n}=37, \mathrm{~N}=2 ; R I M-B P 2^{-/} \mathrm{n}=49, \mathrm{~N}=2$. [E] RIM$B P 2^{++} \mathrm{n}=127, \mathrm{~N}=2 ; R I M-B P 2^{-/} \mathrm{n}=124, \mathrm{~N}=2 ;[\mathrm{D}, \mathrm{E}]$ Mean $\pm \mathrm{SEM}$ and statistical $p$-values are displayed in Table 2. Significance levels: n.s. $p \geq 0.05,{ }^{*} p<0.05,{ }^{* \star} p<0.01,{ }^{* * *} p<0.001$; $\mathrm{n}=$ number of $\mathrm{AZs}, \mathrm{N}=$ number of mice; age of mice: $\mathrm{p} 21-\mathrm{p} 23$. Panels $[A-E]$ adapted from Krinner et al., 2017. 
The majority of clusters formed a "stripe- or double stripe-like" arrangement (66\%), $12 \%$ of $\mathrm{Ca}^{2+}$-channel clusters formed "round" and $22 \%$ "complex" cluster shapes. In RIM-BP2-AZs classes of shapes and their representation were similar with a trend towards less stripeshaped $(62 \%)$ but more round (16\%) appearance. This classification applies to apparent shapes of synapses seemingly laying en face in the imaging plane; however the clusters were not assessed with 3D STED resolution.

Dimensions of "stripe-shaped" $\mathrm{Ca}^{2+}$-channel clusters were quantified using a genetic 2dimensional (2D) Gaussian function fit algorithm (Rio and Pareschi, 2001) to STED images of stripe-shaped $\mathrm{Ca}^{2+}$-channel clusters (illustrated in Figure $15 \mathrm{C}$ ). A significant reduction was observed for the long axes' FWHM ( $p=0.009)$, the area ( $22 \%$ reduction, $p=0.01)$ and integral ( $51 \%$ reduction, $p<0.0001)$ of 2D Gaussian fits to immunofluorescence from Cav1.3 $\mathrm{Ca}^{2+}$-channel clusters in RIM-BP2-- AZs, whereas the short axes' FWHM was unaltered $(p=0.1)$ (Figure 15D, Table 4). Consequently, there was a trend towards a smaller FWHM ratio of long and short axes, which, however, did not reach statistical significance $(p=0.1$, Figure 15D, Table 4). The area and integral calculated from the 2D Gaussian fit function were interpreted as measure of $\mathrm{Ca}^{2+}$-channel cluster size and number of $\mathrm{Ca}^{2+}$-channels in the cluster, respectively. However, one should note that the correlation between measured fluorescence and the number of $\mathrm{Ca}^{2+}$-channels might not be linear, thus does not provide a direct measure for $\mathrm{Ca}^{2+}$-channel numbers, but might serve as a proxy for relative $\mathrm{Ca}^{2+}$ channel levels. In summary, the morphological STED data suggest a rounder and smaller cluster shape with a $51 \%$ reduced integrated Cav1.3 immunofluorescence for stripe-shaped $\mathrm{Ca}^{2+}$-channel clusters of RIM-BP2 deficient AZs (Figure 15D, Table 4). This is consistent with the reduction by $41 \%$ of the $\Delta F / F_{0}$ found by $\mathrm{Ca}^{2+-i m a g i n g ~(F i g u r e ~} 14 \mathrm{~B}$ ) and suggests a reduction in the number of especially synaptic $\mathrm{Ca}^{2+}$-channels upon genetic RIM-BP2 disruption.

The same analysis (illustrated in Figure 15C) was performed for the RIM-BP2 interaction partner bassoon to investigate whether the reduction in clustered $\mathrm{Ca}^{2+}$-channels was mediated directly by RIM-BP2 or potentially indirectly through bassoon interaction. In both $R I M-B P 2^{+/+}$and $R I M-B P 2^{-/} I H C s$, bassoon formed stripe like clusters (Figure 15B). Quantification of clusters (Figure 15C, Table 4) revealed no difference in the FWHM of the long and short axis, long/short axis ratio and cluster area between the two genotypes. However, a significant increase in Bassoon cluster intensity (21\%) was found in RIM$B P 2^{-1}$ AZs (Figure 15D, Table 4), which could potentially indicate a compensatory upregulation of synaptic bassoon levels. Overall, these data indicate that RIM-BP2 regulates $\mathrm{Cav} 1.3 \mathrm{Ca}^{2+}$-channel clustering at IHC ribbon synapses probably downstream of bassoon. 
Table 4: Summary of Cav1.3 and bassoon STED image quantification data

\begin{tabular}{|c|c|c|c|c|c|}
\hline & $\begin{array}{l}\text { FWHM long } \\
\text { axis }(n m)\end{array}$ & $\begin{array}{l}\text { FWHM short } \\
\text { axis }(\mathrm{nm})\end{array}$ & $\begin{array}{l}\text { Ratio } \\
\text { long/short } \\
\text { axis }\end{array}$ & $\begin{array}{l}\text { Area } \\
\left(\mu \mathrm{m}^{2}\right)\end{array}$ & $\begin{array}{l}\text { Integral } \\
\left(\mu \mathrm{m}^{2} \mathrm{x}\right. \\
\text { a.u. })\end{array}$ \\
\hline $\begin{array}{c}\text { Cav1.3 } \\
\text { RIM-BP2 } 2^{+/+} \\
(n=37, N=2)\end{array}$ & $403 \pm 20$ & $221 \pm 8$ & $1.85 \pm 0.09$ & $0.072 \pm 0.005$ & $18.3 \pm 1.7$ \\
\hline $\begin{array}{c}\text { Cav1.3 } \\
R I M-B P 2^{--} \\
(n=49, N=2)\end{array}$ & $338 \pm 13$ & $205 \pm 6$ & $1.67 \pm 0.06$ & $0.056 \pm 0.003$ & $8.98 \pm 0.9$ \\
\hline $\begin{array}{l}\text { Cav1.3 } \\
p \text {-value }\end{array}$ & $\begin{array}{c}0.009 \\
\text { Wilcoxon } \\
\text { rank test } \\
\end{array}$ & $\begin{array}{c}0.1 \\
\text { Studen't t- } \\
\text { test }\end{array}$ & $\begin{array}{c}0.1 \\
\text { Wilcoxon } \\
\text { rank test }\end{array}$ & $\begin{array}{l}0.01 \\
\text { Studen't t-test }\end{array}$ & $\begin{array}{c}7.2 \mathrm{e}-8 \\
\text { Wilcoxon } \\
\text { rank test }\end{array}$ \\
\hline $\begin{array}{c}\text { Bassoon RIM- } \\
\text { BP2 }^{+/+} \\
(n=127, N=2)\end{array}$ & $419 \pm 10$ & $251 \pm 5$ & $1.69 \pm 0.03$ & $0.035 \pm 0.003$ & $11.0 \pm 0.5$ \\
\hline $\begin{array}{c}\text { Bassoon RIM- } \\
\text { BP2 } \\
(n=124, N=2)\end{array}$ & $420 \pm 10$ & $249 \pm 5$ & $1.73 \pm 0.04$ & $0.032 \pm 0.003$ & $14.0 \pm 0.8$ \\
\hline $\begin{array}{l}\text { Bassoon } \\
p \text {-value }\end{array}$ & $\begin{array}{c}0.9 \\
\text { Student's t- } \\
\text { test }\end{array}$ & $\begin{array}{c}0.8 \\
\text { Student's t- } \\
\text { test }\end{array}$ & $\begin{array}{c}0.8 \\
\text { Wilcoxon } \\
\text { rank test }\end{array}$ & $\begin{array}{c}0.8 \\
\text { Student's t- } \\
\text { test }\end{array}$ & $\begin{array}{c}0.01 \\
\text { Wilcoxon } \\
\text { rank test }\end{array}$ \\
\hline
\end{tabular}

Summary of grant average data (mean \pm SEM) from 2-color 2D STED images of immunofluorescently labeled stripe-shaped $\mathrm{Ca}_{v} 1.3 \mathrm{Ca}^{2+}$-channel clusters or bassoon clusters (Figure 15). Fit amplitudes and FWHM of the long and short axis of a 2D Gaussian fit function were used for quantitative analysis. Further, the ratio of long over short axis, cluster area and integral were calculated. $p$-values and statistical test are depicted for each dataset; significance levels: n.s. $p \geq 0.05,{ }^{*} p<0.05,{ }^{* *} p<0.01,{ }^{* * *} p<0.001 ; \mathrm{n}=$ numbers of $\mathrm{AZs}$, $\mathrm{N}=$ number of mice; age of mice: p21-p23. Table adapted from Krinner et al., 2017.

\section{RIM-BP2 promotes IHC exocytosis}

The expression pattern of RIM-BP2 in IHC AZs (Figure 12C) and recent literature do not only suggest a link between RIM-BP2 and $\mathrm{Ca}^{2+}$-channels, but also to SVs potentially via interaction with bassoon or Rab3/RIM (Acuna et al. 2015; Acuna, Liu, and Südhof 2016; Davydova et al. 2014; Grauel et al. 2016; Hibino et al. 2002; Liu et al. 2011; M. Müller, Genç, and Davis 2015; Y. Wang et al. 2002). Hence, it was tested if RIM-BP2 regulates synaptic transmission, and whether such regulation could be mediated either via its promotion of $\mathrm{Ca}^{2+}$-channel abundance, via a role in coupling of $\mathrm{Ca}^{2+}$-channels to vesicular release sites, or by directly affecting SV release.

For that, the perforated patch-clamp technique was employed, as it provides endogenous buffering conditions to measuring exocytic membrane capacitance changes $\left(\Delta \mathrm{C}_{\mathrm{m}}\right)$ of the $\mathrm{IHC}$ in response to voltage-gated $\mathrm{Ca}^{2+}$-influx during step-depolarizations of various 
durations (Figure 16A). IHC exocytosis can be described by two kinetic components, representing different SV release phases (Moser and Beutner, 2000). The RRP of SVs was described by a fast and saturating component that can be approximated by the exocytic $\Delta \mathrm{C}_{\mathrm{m}}$ response of IHCs in response to $20 \mathrm{~ms}$ long depolarizations. RIM-BP2 disruption tended to reduce RRP exocytosis (by $21 \%$ for $\Delta \mathrm{C}_{\mathrm{m}}$ elicited by $20 \mathrm{~ms}$ depolarization, but not reaching statistical significance: $p=0.1$ ) (Figure $16 \mathrm{~A}$ ). The reduction in exocytosis in the absence of RIM-BP2 became significant for the slower linear component of exocytosis (50 ms depolarization, $38 \%, p=0.0007$ ), which was attributed to the sustained phase of SV release, involving SV resupply after RRP depletion (Goutman and Glowatzki, 2007; Meyer et al., 2009; Pangršič et al., 2010; Schnee et al., 2005). To confirm this result, a line was fit to the sustained phase of exocytosis (from 50 to $200 \mathrm{~ms}$ ) to estimate the kinetics of vesicle turnover during ongoing stimulation (Figure 16A, dashed line in top left panel). In RIM-BP2 - IHCs, SV turnover rates were significantly slower (193 fF/s) compared to RIM-BP2 ${ }^{+/+} \mathrm{IHCs}$ (279 fF/s) ( $p=0.001$, Figure 16A right panel).

In addition to the reduction in exocytosis during the sustained phase of SV release, a significant reduction in the whole-cell voltage-gated $\mathrm{Ca}^{2+}$-influx (integrated $\mathrm{Ca}^{2+}$-charge, $\mathrm{Q}_{\mathrm{Ca}}$ ) was observed during all IHC depolarization durations (Figure 16A, bottom). This result is in line with the reduced $I_{C a}$ amplitude measured in $\mathrm{Ca}^{2+} \mathrm{IV}$ recordings (Figure 13A), as well as with the suggested reduction in the number of synaptic $\mathrm{Ca}^{2+}$-channels (Figure 14 and 15) in RIM-BP2 deficient IHCs.

Since not only exocytosis, but also the $\mathrm{Ca}^{2+}$ influx was significantly reduced, it was next tested whether the reduction in whole-cell $\mathrm{Ca}^{2+}$-current alone could explain the discrepancy in exocytosis between the $R I M-B P 2^{+/+}$and $R I M-B P 2^{-/} I H C s$. For that the relation between the exocytic $\Delta \mathrm{C}_{\mathrm{m}}$ and its corresponding $\mathrm{Ca}^{2+}$-charge $\left(\mathrm{Q}_{\mathrm{ca}}\right)$ was investigated as the ratio between $\Delta \mathrm{C}_{\mathrm{m}}$ and $\mathrm{Q}_{\mathrm{Ca}}$ for $20 \mathrm{~ms}$ step-depolarizations to assess the efficiency of $\mathrm{Ca}^{2+}$-influx to drive RRP exocytosis and for 50 to $200 \mathrm{~ms}$ step-depolarizations to assess the sustained phase of exocytosis (Figure $16 \mathrm{~B}$ ). The $\Delta \mathrm{C}_{\mathrm{m}} / \mathrm{Q}_{\mathrm{ca}}$ ratio was comparable between both genotypes for 20 ms depolarizations $(p=0.9)$. Nevertheless, there was indeed a significant difference between the two genotypes regarding the sustained phase of exocytosis (50 ms: $p=0.003,100 \mathrm{~ms}: p=0.04,200 \mathrm{~ms}: p=0.01)$. These results indicate that the reduction in RRP exocytosis could be the consequence of reduced $\mathrm{Ca}^{2+}$-influx, whereas the discrepancy of the sustained exocytic responses exceeds what can be explained by the reduction in $\mathrm{Ca}^{2+}$ influx pointing towards an additional role of RIM-BP2 in sustained SV exocytosis.

For further analysis, the extracellular $\left[\mathrm{Ca}^{2+}\right]_{\mathrm{e}}$ was elevated from 2 to $5 \mathrm{mM}$, thereby increasing single channel $\mathrm{Ca}^{2+}$-current (Figure 16B). As consequence the ratio between $\triangle \mathrm{C}_{\mathrm{m}}$ and $\mathrm{Q}_{\mathrm{Ca}}$ in $R I M-B P 2^{-/} \mathrm{IHCs}$ was elevated so that it was indistinguishable from RIM$B P 2^{+++}$levels at $2 \mathrm{mM}\left[\mathrm{Ca}^{2+}\right]_{\mathrm{e}}(50 \mathrm{~ms}: p=0.3,100 \mathrm{~ms}: p=0.9,200 \mathrm{~ms}: p=0.4)$. This suggests 
that boosting single $\mathrm{Ca}^{2+}$-channel current can overcome the alteration imposed by RIM-BP2 disruption, potentially because $\mathrm{Ca}^{2+}$-domains around channels spread further out, reaching more remotely positioned fusion-competent vesicles.
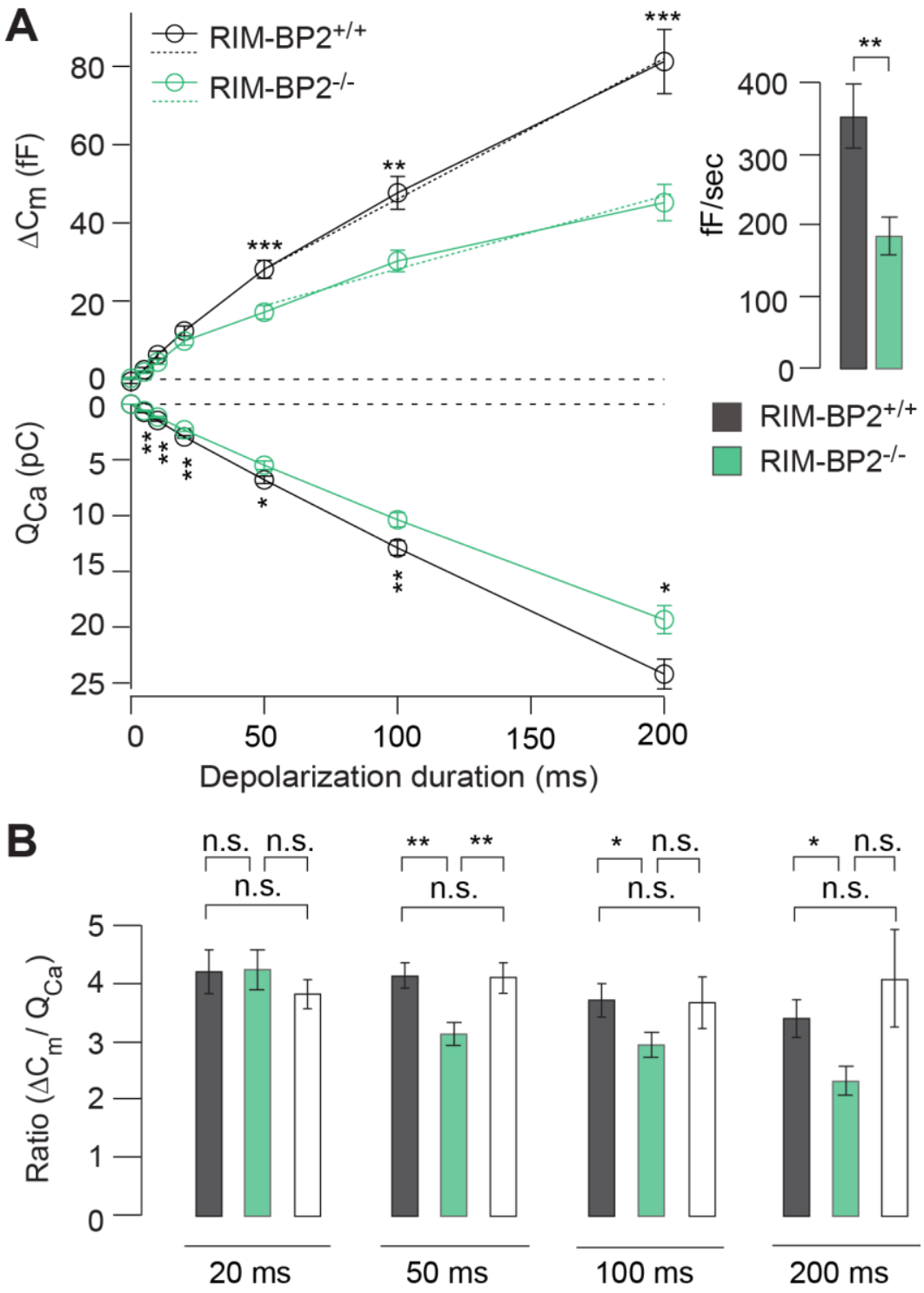

RIM-BP2 $\left.{ }^{+/+}\right] 2 \mathrm{mM}\left[\mathrm{Ca}^{2+}\right]_{\mathrm{e}}$
$\begin{aligned} & \text { RIM-BP2-/- } \\ & \text { RIM-BP2-/- }\end{aligned} 5 \mathrm{mM}\left[\mathrm{Ca}^{2+}\right]_{e}$

Figure 16: RIM-BP2 promotes sustained exocytosis in IHCs

[A] Left: Bookman plot showing exocytic membrane capacitance changes ( $\Delta \mathrm{C}_{\mathrm{m}}$, top) and corresponding whole-cell $\mathrm{Ca}^{2+}$-current integrals $\left(\mathrm{Q}_{\mathrm{Ca}}\right.$, bottom) of RIM-BP2 deficient (RIM$B P 2^{--}, \mathrm{n}=10$, green) and control $\left(R I M-B P 2^{+/+}, \mathrm{n}=12\right.$, black) IHCs for various depolarization durations to $-14 \mathrm{mV}$. $\mathrm{Q}_{\mathrm{Ca}}$ was significantly reduced during all depolarization durations ( $p=0.01$ to 0.002 ). Exocytic $\Delta \mathrm{C}_{\mathrm{m}}$ was significantly reduced in $R I M-B P 2^{-/} \mathrm{IHCs}$ for 50 $(p=0.0007), 100(p=0.002)$, and $200 \mathrm{~ms}(p=0.0007)$ depolarizations representing the sustained phase of SV release, whose rate is governed by vesicle resupply to the RRP. A line was fit to the sustained phase of exocytosis to each individual IHC (from 50 to $200 \mathrm{~ms}$, 
dashed line) to estimate the kinetics of vesicle turnover. Right: IHC grand average (mean \pm SEM) of line fit slopes as estimate for SV turnover rates. A significant reduction was

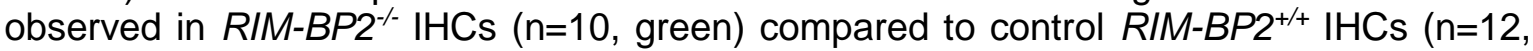
black) (Wilcoxon rank test, $p=0.001$ ). [B] The relation between the exocytic $\Delta \mathrm{C}_{\mathrm{m}}$ and its corresponding integrated $\mathrm{Ca}^{2+}$-current $\left(\mathrm{Q}_{\mathrm{ca}}\right)$ was calculated as the ratio between $\Delta \mathrm{C}_{\mathrm{m}}$ and $\mathrm{Q}_{\mathrm{Ca}}$ from the data shown in the bookman plot $[\mathrm{A}]$. A significant difference between the two genotypes (RIM-BP2 $2^{++}, \mathrm{n}=12$, black bars; RIM-BP2 $2^{-/}, \mathrm{n}=10$, green bars) was observed for the sustained phase of exocytosis (50-200 ms, $p=0.01$ to 0.003 ), but not for RRP exocytosis (20 ms, $p=0.9$ ). After the extracellular $\left[\mathrm{Ca}^{2+}\right]_{\mathrm{e}}$ was elevated from 2 to $5 \mathrm{mM}$ in $R I M-B P 2^{-1-}$ IHCs ( $\mathrm{n}=7$, white bars), the ratio between $\Delta \mathrm{C}_{\mathrm{m}}$ and $\mathrm{Q}_{\mathrm{Ca}}$ was indistinguishable from RIM$B P 2^{+/+}$levels ( $p=0.4$ to 0.9). [A-B] Data information: Data represent IHC grand averages, mean \pm SEM; Student's t-test unless specified differently (see above), Significance levels: n.s. $p \geq 0.05,{ }^{*} p<0.05,{ }^{* *} p<0.01,{ }^{* *} p<0.001 ; n=$ number of IHCs; age of mice: $p 14-p 16$. Panels [A-B] adapted from Krinner et al., 2017.

\section{RIM-BP2 does not regulate $\mathrm{Ca}^{2+}$-influx - exocytosis coupling of RRP SVs in IHCs}

Since the reduction in exocytosis could not be fully explained by the reduction of presynaptic $\mathrm{Ca}^{2+}$-influx alone, a looser $\mathrm{Ca}^{2+}$-influx-exocytosis coupling was considered. $\mathrm{Ca}^{2+}$-influx exocytosis coupling was investigated by estimating the apparent $\mathrm{Ca}^{2+}$ cooperativity $m$ of exocytosis (Augustine et al., 1991) (Dr. Sangyong Jung contributed to this data set with $n=3$ IHC recordings for each genotype). The number of open $\mathrm{Ca}^{2+}$-channels was gradually reduced during perforated patch-clamp experiments by slow perfusion of the dihydropyridine channel antagonist isradipine $(10 \mu \mathrm{M})$ and $20 \mathrm{~ms}$ depolarizations separated by at least $60 \mathrm{sec}$ were repeatedly applied for SV-replenishment to secure complete RRP recovery. The apparent $\mathrm{Ca}^{2+}$ cooperativity $m$ was obtained by fitting the relationship of exocytic capacitance changes $\left(\Delta \mathrm{C}_{\mathrm{m}}, \mathrm{y}\right.$-axis) and the corresponding integrated $\mathrm{Ca}^{2+}$-charge $\left(Q_{C a}, \mathrm{X}\right.$-axis) for each individual IHC with a power function $\left(\Delta C_{m}=A \times\left(Q_{C a}\right)^{m}\right)$ restricted to the range that did not show obvious saturation of $\Delta \mathrm{C}_{\mathrm{m}}$ (Figure 17B).

Previously $m$ was found to be close to unity for IHCs after the onset of hearing, indicating $\mathrm{Ca}^{2+}$ nanodomain-like control of exocytosis to govern synaptic sound encoding (Figure 17A, yellow). For immature IHCs however, $m$ was found $>1$ indicating a supralinear $\mathrm{Ca}^{2+}$ micodomain-like control of exocytosis (Figure 17A, orange) (Brandt et al., 2005; Jung et al., 2015a; Wong et al., 2014). If RIM-BP2 was a molecular linker regulating the distance between the Cav1.3 $\mathrm{Ca}^{2+}$-channel complex and the vesicular release site, essential for establishing this $\mathrm{Ca}^{2+}$ nanodomain-like control, one would expect an increase in $m$ upon RIM-BP2 disruption. However, the estimates of $m$ were statistically indistinguishable between $R I M-B P 2^{+/+}$and RIM-BP2/- IHCs $(1.55 \pm 0.044$ vs. $1.64 \pm 0.17$, mean \pm SEM, $p=0.5$ ), indicating that RIM-BP2 is not required for the tight $\mathrm{Ca}^{2+}$ nanodomain-like coupling of readily releasable SVs to the $\mathrm{Ca}^{2+}$-channel, at least if SVs were replenished in due time. 
A

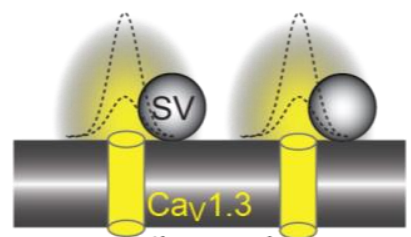

presynaptic membrane

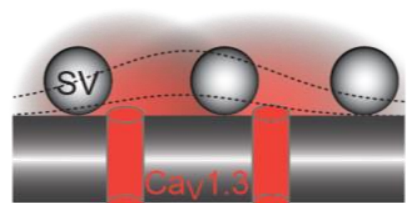

presynaptic membrane

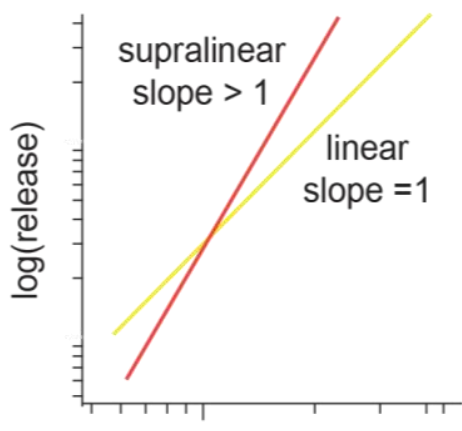

$\log \left(\mathrm{Ca}^{2+}\right.$ influx $)$

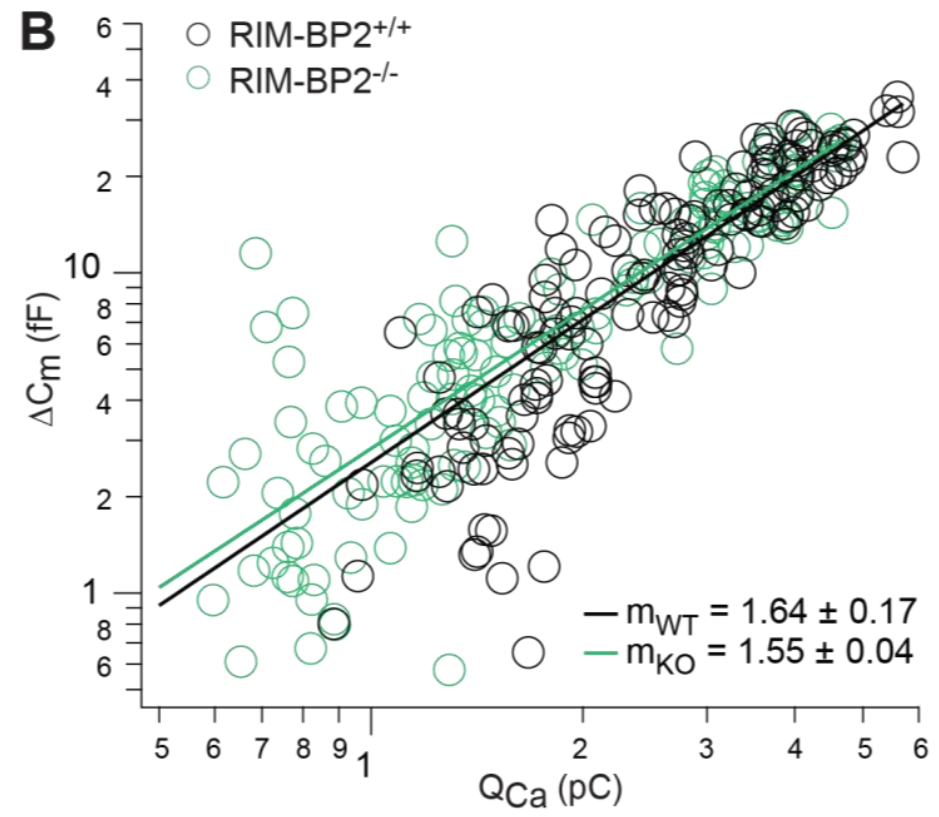

Figure 17: RIM-BP2 disruption did not affect IHC Ca ${ }^{2+}$-influx - exocytosis coupling

[A] Left, top: After the onset of hearing SV fusion seems under $\mathrm{Ca}^{2+}$ nanodomain-like control, where the release of each SV would be controlled by a single $\mathrm{Ca}^{2+}$-channel. Left, bottom: Before the onset of hearing SV fusion seems under $\mathrm{Ca}^{2+}$ microdomain-like control, where the release of each SV would be controlled by several $\mathrm{Ca}^{2+}$-channels. Right: $\mathrm{Ca}^{2+}$ influx - exocytosis coupling can be assessed through the apparent $\mathrm{Ca}^{2+}$ cooperativity $m$ of exocytosis. $m$ is estimated by reducing the number of open $\mathrm{Ca}^{2+}$-channels during patchclamp experiments by slow perfusion of a channel blocker, e.g. the dihydropyridine channel antagonist isradipine $(10 \mu \mathrm{M})$. The relationship of $\mathrm{Ca}^{2+}$-influx - exocytosis is plotted in a double logarithmic scale. For $\mathrm{Ca}^{2+}$ nanodomain-like control in mature IHCs, $m$ was found to be close to unity (yellow line). For immature $\mathrm{IHCs} m$ was found to be $>1$, describing a supralinear micodomain-like (orange line) coupling. [B] Double-logarithmic plot of exocytic membrane capacitance changes $\left(\Delta \mathrm{C}_{\mathrm{m}}\right)$ and corresponding whole-cell $\mathrm{Ca}^{2+}$-current integrals $\left(\mathrm{Q}_{\mathrm{Ca}}\right)$ during $20 \mathrm{~ms}$ depolarizations and perfusion of IHCs with isradipine to gradually decrease the number of open $\mathrm{Ca}^{2+}$-channels. Symbols represent individual data points from depolarizations of RIM-BP2 deficient (RIM-BP2 ${ }^{-1}, \mathrm{n}=7 \mathrm{IHCs}$, each 20 to 43 data points, green) and control IHCs (RIM-BP2 $2^{+/+}, \mathrm{n}=6$, each 20 to 42 data points, black). Solid lines represent best-fit power function to all data points of each genotype. The estimates of $m$ for each individual cell were statistically indistinguishable between genotypes, indicating normal $\mathrm{Ca}^{2+}$ influx-exocytosis coupling ( $p=0.5$, Student's t-test). Dr. Sangyong Jung contributed $\mathrm{IHC}$ recordings to this data set ( $\mathrm{n}=3 \mathrm{IHC}$ recordings for each genotype). Significance levels: n.s. $p \geq 0.05,{ }^{*} p<0.05,{ }^{* *} p<0.01,{ }^{* * *} p<0.001 ; n=$ number of IHCs; age of mice: p14-16. Panel [B] adapted from Krinner et al., 2017. 
Hence, these results do not rule out that RIM-BP2 could serve in promoting the engagement of newly arriving SVs and $\mathrm{Ca}^{2+}$-channels after RRP depletion. Together with the increased $\mathrm{Ca}^{2+}$-dependence of exocytosis during sustained release rates, such a scenario could indeed cause the reduction of sustained exocytosis. Therefore, deficits in replenishing SVs from an upstream vesicle pool were investigated next.

\section{RIM-BP2 facilitates fast SV recovery after RRP depletion}

A paired-pulse paradigm measured vesicle refilling from an upstream vesicle pool into the $\mathrm{RRP}$, using two consecutive $20 \mathrm{~ms}$ depolarization pulses to $-14 \mathrm{mV}$ with variable interstimulus-intervals $(\Delta t)$ (illustrated in Figure $18 \mathrm{~A}$ ). The first $20 \mathrm{~ms}$ pulse was supposed to deplete the RRP, the second $20 \mathrm{~ms}$ pulse was supposed to probe RRP recovery within variable intervals to the first pulse.
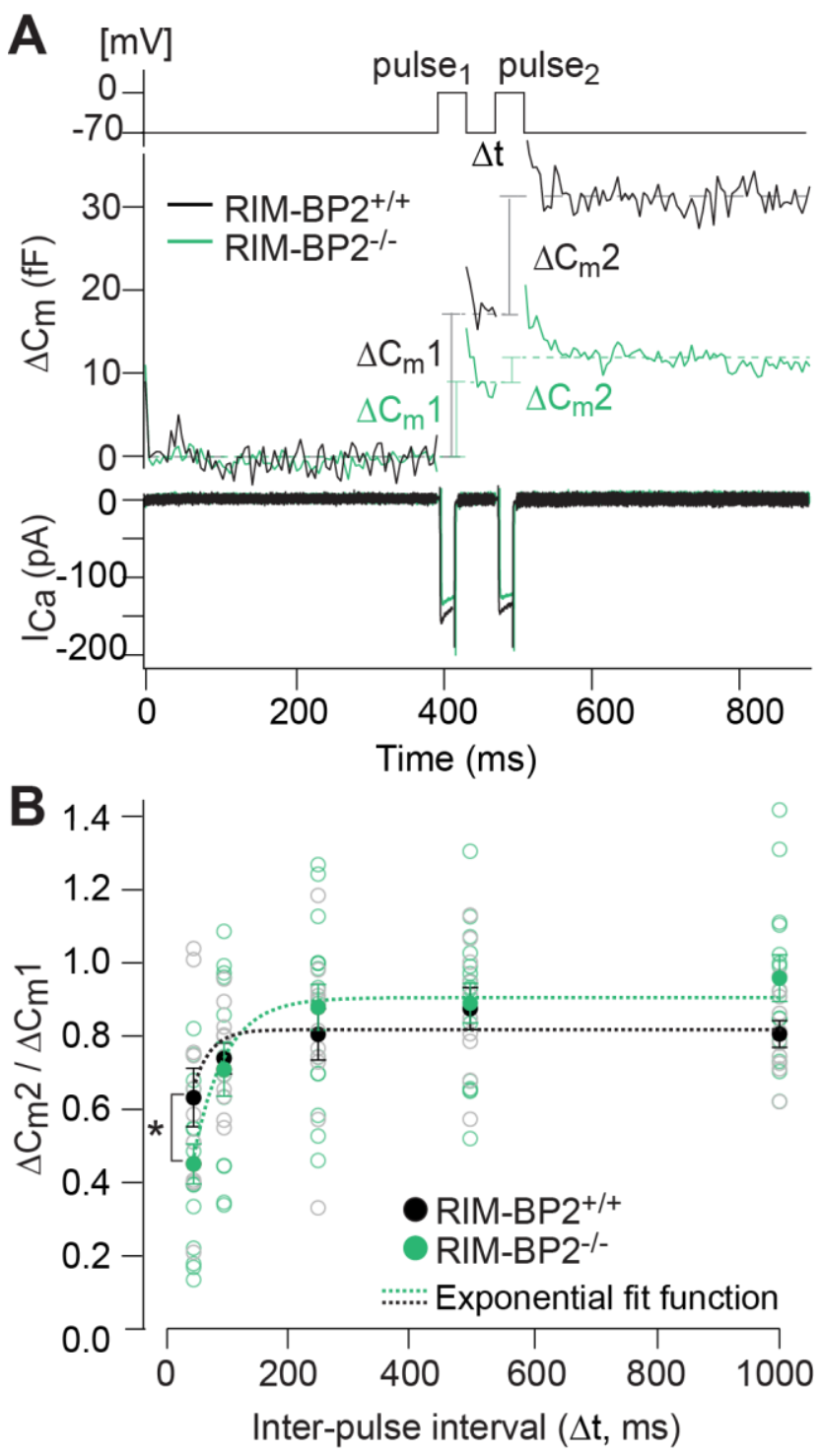

Figure 18: RIM-BP2 facilitates fast recruitment of SVs after RRP depletion in IHCs 
[A] A paired-pulse protocol was used to assess SV-replenishment after RRP depletion. Average exocytic membrane capacitance change traces $\left(\Delta \mathrm{C}_{\mathrm{m}}\right.$, middle) and corresponding $\mathrm{Ca}^{2+}$-currents $\left(I_{C a}\right.$, bottom) of $R I M-B P 2^{-/}\left(\mathrm{n}=18\right.$, green) and control IHCs $\left(R I M-B P 2^{+/}, \mathrm{n}=14\right.$, black) in response to a pair of $20 \mathrm{~ms}$ depolarizations separated by an inter-pulse interval $(\Delta t)$ of $50 \mathrm{~ms}$ (top). SV-replenishment was measured as the paired-pulse ratio between the second and the first exocytic membrane capacitance change $\left(\Delta \mathrm{C}_{\mathrm{m}} 2 / \Delta \mathrm{C}_{\mathrm{m}} 1\right)$. [B] Pairedpulse ratios $\left(\Delta \mathrm{C}_{\mathrm{m}} 2 / \Delta \mathrm{C}_{\mathrm{m}} 1\right)$ at varying inter-pulse intervals $(\Delta t)$ were significantly reduced in $R I M-B P 2^{-/}$IHCs ( $\mathrm{n}=18$, green) compared to control IHCs $\left(R I M-B P 2^{+/+}, \mathrm{n}=14\right.$, black) for RRP recovery times of $50 \mathrm{~ms}(p=0.02)$. Longer RRP recovery times $(>50 \mathrm{~ms})$ were sufficient for RRP-replenishment in RIM-BP2 deficient IHCs ( $p=0.1$ to 0.8$)$. The time course of RRP recovery was estimated by fitting the paired-pulse data with a single exponential fit function (dashed lines). $\tau$ was significantly slower in RIM-BP2 ${ }^{-\alpha}$ IHCs (mean \pm SEM: $107 \pm 24 \mathrm{~ms}$, $\mathrm{n}=5$, green) compared to $R I M-B P 2^{+/+} \mathrm{IHCs}$ (mean \pm SEM: $44 \pm 7 \mathrm{~ms}, \mathrm{n}=5$, black, $p=0.03$, Student's t-test). Data represent IHC averages (empty circles) and grand averages (filled circles, dashed lines), mean \pm SEM. Student's t-test: n.s. $p \geq 0.05,{ }^{*} p<0.05,{ }^{* *} p<0.01$, ${ }^{* *} p<0.001$. [A, C] $n=$ number of IHCs; age of mice: $p 14-p 16$. Panels [A-B] adapted from Krinner et al., 2017.

RRP recovery was slowed in $R I M-B P 2^{-1} \mathrm{IHCs}$, as is evident from significantly smaller responses at an interval of $50 \mathrm{~ms}(p=0.02)$, likely representing a fast recycling SV pool. Longer RRP recovery times (>50 ms) were sufficient for RRP-replenishment in RIM-BP2 deficient IHCs (Figure 18B). The time course of RRP recovery was estimated by fitting the paired-pulse data with a single exponential fit function to a subset of recordings where sufficient data was collected to allow reliable fitting (Figure 18B, dashed lines). The RRP refilling time constant $\left(\tau\right.$, mean \pm SEM) was slower in $R I M-B P 2^{-\alpha}$ IHCs $(107 \pm 24 \mathrm{~ms})$ compared to $R I M-B P 2^{+++}$IHCs $(44 \pm 7 \mathrm{~ms})(p=0.03)$. This indicates that RIM-BP2 facilitates fast recruitment of SVs after RRP depletion in IHC ribbon synapses. Speculatively, RIMBP2 could be involved in a fast dynamic process of guiding SVs into close proximity to a nearby $\mathrm{Ca}^{2+}$-channel. Longer RRP recovery times ( $>50 \mathrm{~ms}$ ), however, were sufficient for normal RRP-replenishment in RIM-BP2 deficient IHCs (Figure 18B), likely because of the increasing probability of alternative protein-protein interactions taking place providing the proper positioning between the replenished SVs and their release site.

\section{RIM-BP2 regulates the distance of SVs to the presynaptic IHC membrane}

The effect of RIM-BP2 disruption on the ultrastructural level of IHC ribbon synapses was examined by transmission electron tomography (tomography and $3 \mathrm{D}$ tomogram reconstructions were performed by Prof. Dr. Carolin Wichmann). Reconstructed virtual tomogram sections and the respective $3 \mathrm{D}$ tomogram models of representative ribbon synapses of $R I M-B P 2^{-/}$and $R I M-B P 2^{+/+} I H C$ s are displayed in Figure $19 \mathrm{~A}$. The overall synapse ultrastructure in RIM-BP2-/ IHCs was normal (Figure 19A): Synaptic ribbons (red) were anchored to the presynaptic membrane (blue) via a PD (pink) and the size of synaptic ribbons and the PDs were statistically indistinguishable from $R I M-B P 2^{+/+} I H C s$ (Figure 19B, 
left). SVs were classified into three pools. Those SVs that appeared directly adjacent to the ribbon (within $80 \mathrm{~nm}$ ) within the first SV row were considered as RA-SVs (Figure 19A, green). Those SVs that were directly facing the AZ membrane (within $100 \mathrm{~nm}$ from the PD and within $50 \mathrm{~nm}$ from the presynaptic plasma membrane) were classified as MP-SVs (Figure 19A, MP-SVs, yellow) (Jung et al., 2015a). SVs that fit both criteria were classified as MP-SVs. As consequence, RA-SVs comprise only those SVs that are more than $50 \mathrm{~nm}$ away from the plasma membrane (Figure 19A). SVs that were in close proximity to the plasma membrane $(\leq 50 \mathrm{~nm}$ ) and the area marked by the postsynaptic density, but not within $100 \mathrm{~nm}$ from the PD were classified as "outlying"-SVs. The number of MP-SVs, RASVs and "outlying"-SVs were unaltered in RIM-BP2/- IHCs (Figure 19B, right).
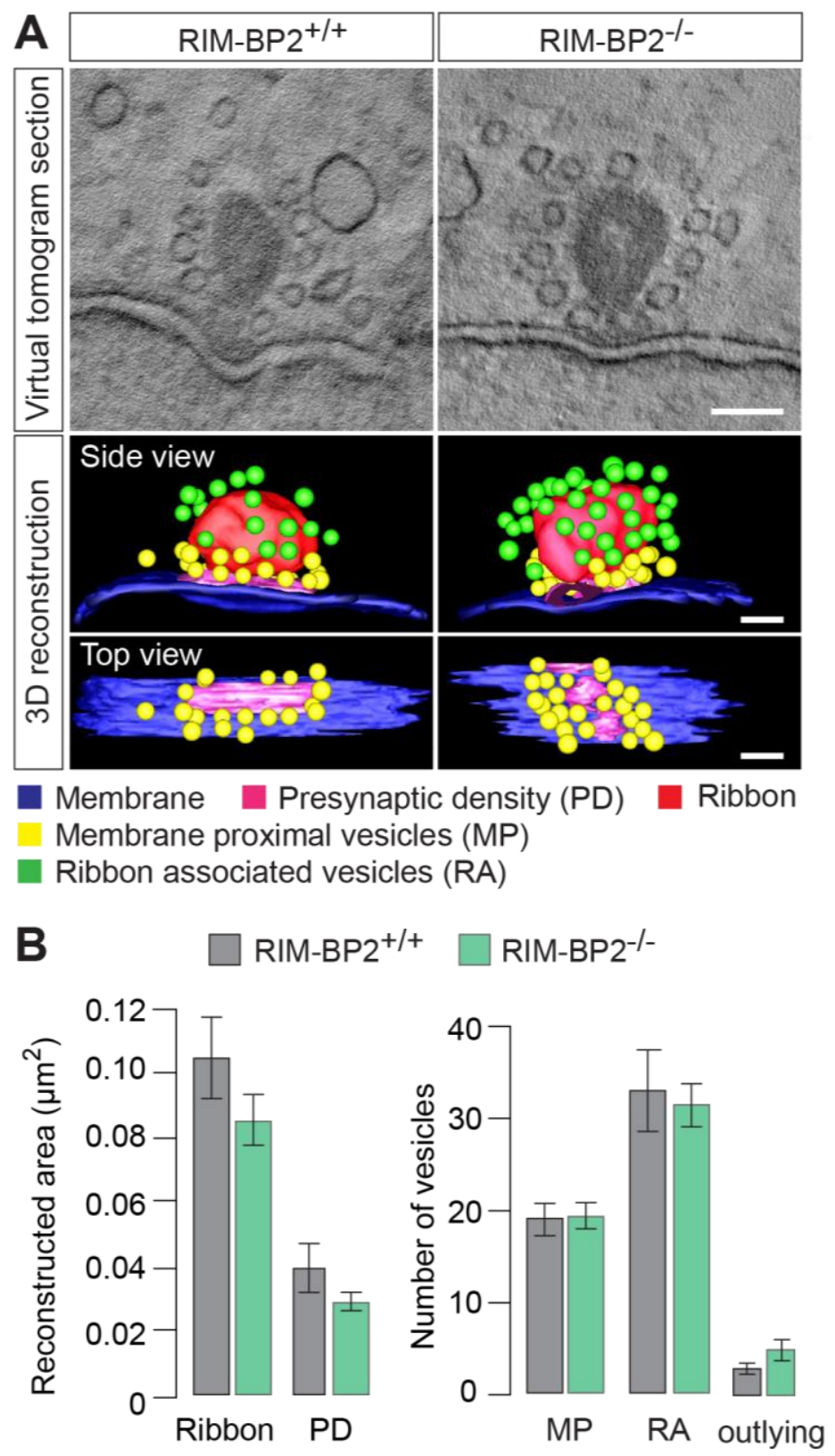

Figure 19: RIM-BP2 does not influence ribbon synapse ultrastructure or the number of SVs at IHC AZs 
[A] Exemplary virtual sections of $R I M-B P 2^{+/+}$and $R I M-B 2^{-/-}$ribbon synapses (top) and side and top view of respective 3D models (bottom). The overall synapse ultrastructure in RIM$B P 2^{-/}$IHCs was normal: Synaptic ribbons (red) were anchored to the presynaptic membrane (blue) via a PD (pink). SVs that were located within $100 \mathrm{~nm}$ distance from the $\mathrm{PD}$ and with a membrane-to-membrane distance of $\leq 50 \mathrm{~nm}$ away from the presynaptic plasma membrane were classified as "membrane proximal" (MP) SVs (yellow). SVs that appeared within $80 \mathrm{~nm}$ around the presynaptic ribbon within the first row of SVs around the ribbon were considered as "ribbon associated" (RA) SVs (green). Scale bar: $100 \mathrm{~nm}$. Prof. Dr. Carolin Wichmann performed tomography and 3D tomogram reconstructions. [B] Quantitative analysis of electron tomograms: In RIM-BP2 ${ }^{-1} A Z s(n=9, N=2)$ no significant differences were found compared to $R I M-B P 2^{++} A Z s(n=9, N=2)$ when comparing reconstructed ribbon area (Student's t-test $p=0.13$ ) and PD area (Wilcoxon rank test $p=0.13$ ) (left), as well as the average number of MP-SVs (Student's t-test $p=0.14$ ), RA-SVs (Student's t-test $p=0.76$ ), or "outlying"-SVs (Student's t-test $p=0.14$ ) (right). Data represent grand averages, mean \pm SEM; Significance levels: n.s. $p \geq 0.05,{ }^{*} p<0.05,{ }^{* *} p<0.01$, ${ }^{* * *} p<0.001 ; n=$ number of tomograms, $\mathrm{N}=$ number of mice; age of mice: $\mathrm{p} 21$. Panels [A-B] partly adapted from Krinner et al., 2017.

Since RIM-BP2 might act as molecular linker between SVs and $\mathrm{Ca}^{2+}$-channels via interaction with bassoon and RIMs/Rab3, the average distance and distribution of MP and "outlying"-SVs regarding their distance to the plasma membrane and the PD was studied in more detail. For that, the shortest (membrane-to-membrane) distance between MP-SVs (Figure 20A) or "outlying"-SVs (Figure 20B) and the plasma membrane or the PD was measured (Figure 20). The cumulative distribution function shows the probability of SVs to be located at a specific distance with respect to the plasma membrane or PD (Figure 20CF). For visualization, the distance of SVs with respect to the plasma membrane or PD was plotted in histograms (Figure 20C-F). The average distance between MP-SVs and the PD and plasma membrane was unaltered (Figure 20A), whereas the distribution of MP-SV with respect to the plasma membrane was changed in the absence of RIM-BP2 (Figure 20C). Based on the cumulative distribution of MP-SVs regarding their distance to the membrane (solid lines), the MP-SV pool was divided into a large fraction (70\%) of SVs within 0-25 nm membrane distance and a small fraction (30\%) of SVs located within 25-50 nm membrane distance. The distributions differed for both fractions of MP-SVs between $R I M-B P 2^{+/+}$and $R I M-B P 2^{-1-}$ AZs (small fraction: $p=0.02$ and large fraction: $p=0.004$ ). There was a tendency of more MP-SVs in RIM-BP2- AZs to populate the smaller distance bins (Figure 20C), while less SVs were observed with intermediate distance ( 20-40 nm). A second accumulation of MP-SVs further away from the membrane ( 40-50 nm) appeared, which seemed absent at $R I M-B P 2^{+/+}$AZs. The normal count of MP-SVs (Figure 19B) and the unaltered lateral distribution of MP-SVs with respect to the PD (Figure 20E) suggests that exocytosis is generally not limited by the availability of SVs. RIM-BP2 might instead regulate the distance of SVs to the plasma membrane, which might be physiologically reflected in fast SV recruitment to the plasma membrane for release. 

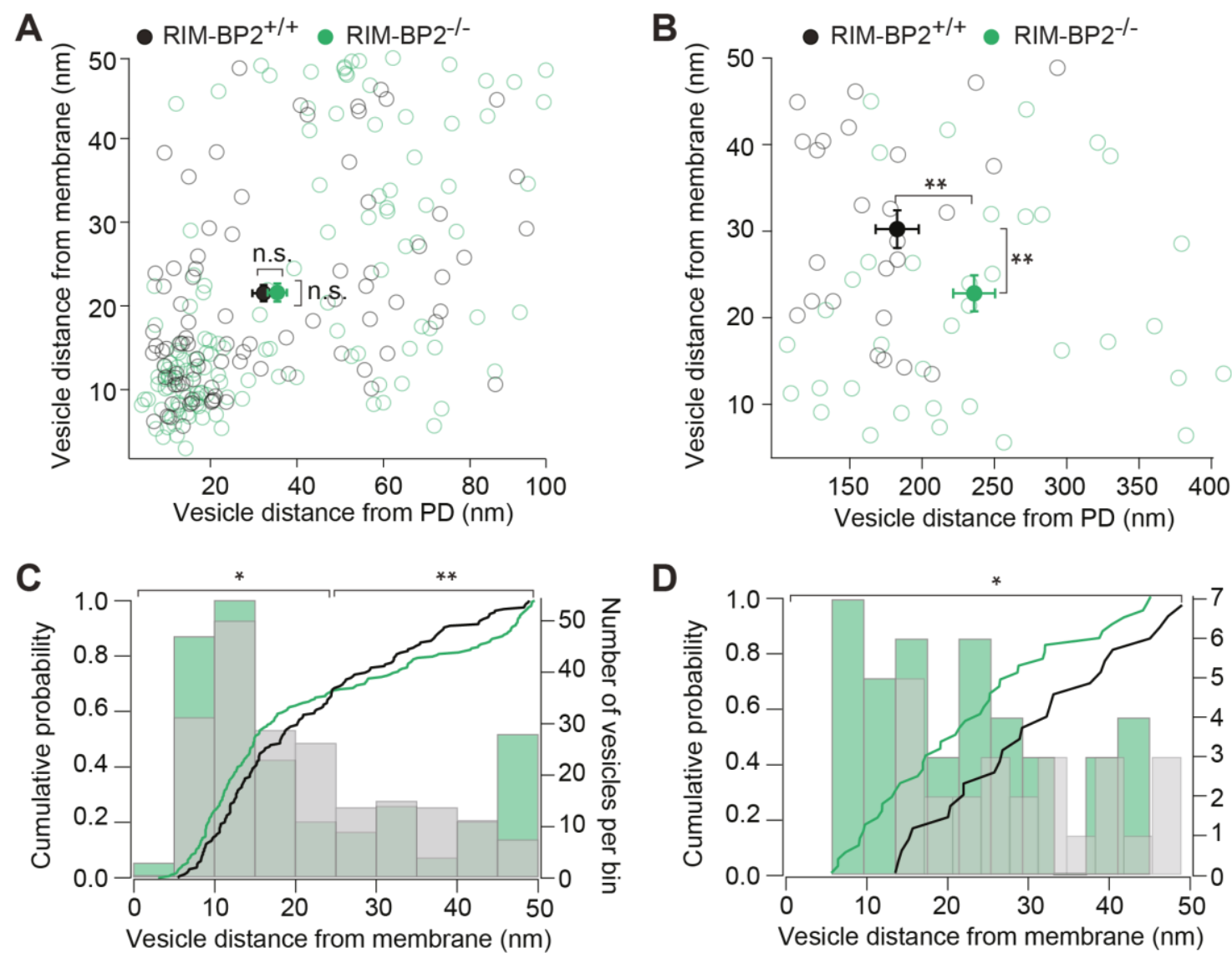

D
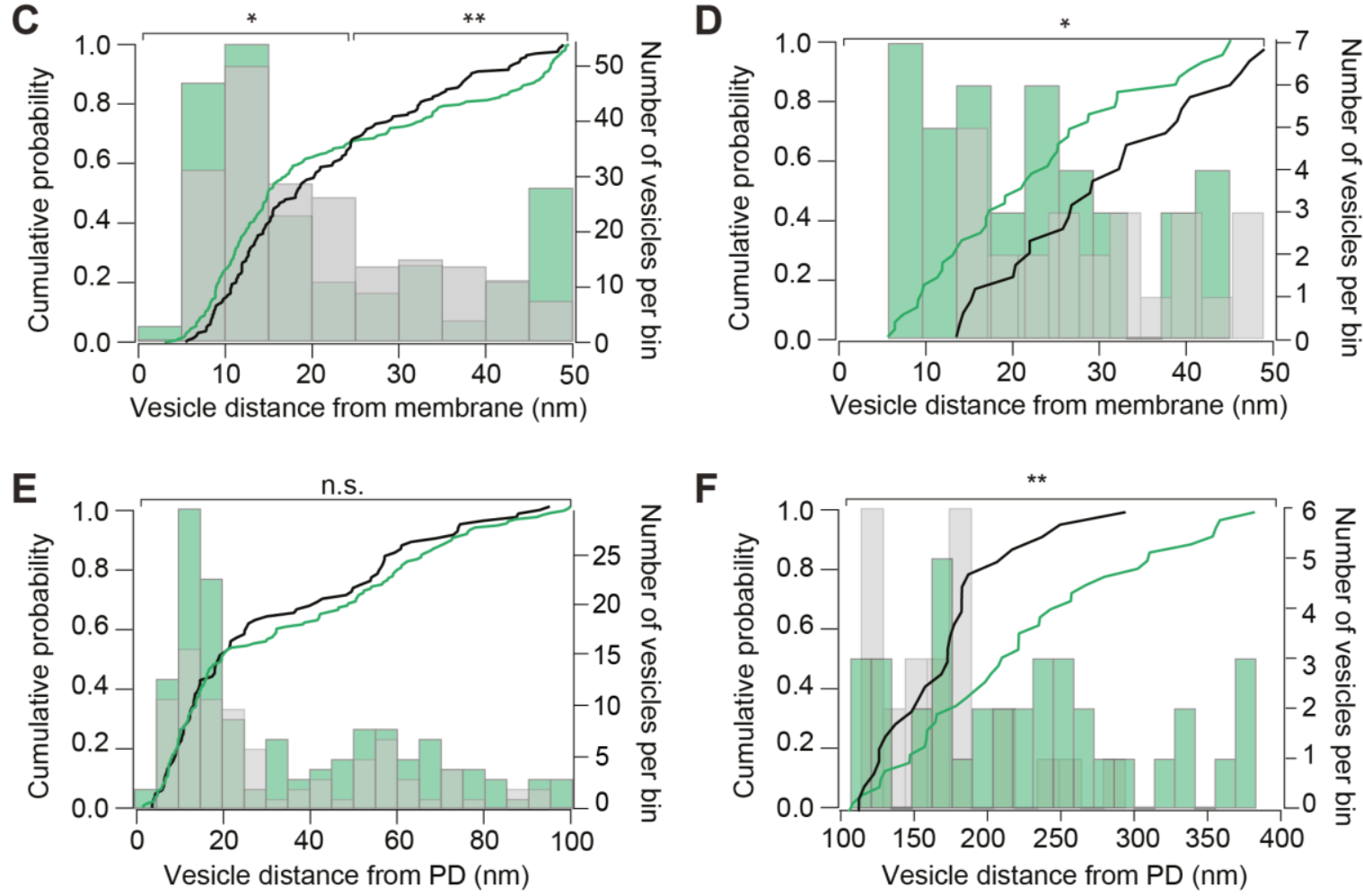

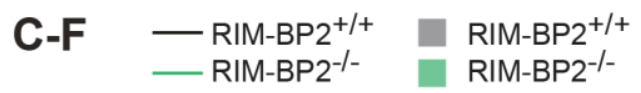

Figure 20: RIM-BP2 regulates the distribution of MP-SVs and "outlying"-SVs

Distribution of MP-SVs $[A, C, E]$ and "outlying"-SVs $[B, D, F]$ regarding their distance to the plasma membrane [C,D] and PD [E,F]. The shortest (membrane-to-membrane) distance between MP- and "outlying"-SVs and the plasma membrane and PD was measured. [A, B] Average (filled circles, mean \pm SEM) and individual SV distance (open circles) to the plasma membrane (y-axis) and PD (x-axis) was plotted for MP-SVs [A] and "outlying"-SVs [B]. [A] On average, the distance of MP-SVs to the plasma membrane (Wilcoxon rank test $p=0.29$ ) and PD (Wilcoxon rank test $p=0.44$ ) was unaltered in $R I M-B P 2^{-/} A Z s$. [B] On average "outlying"-SVs were shifted closer to the plasma membrane (Student's t-test $p=0.005$ ) and further away from the PD (Wilcoxon rank test $p=0.005$ ) in $R I M-B P 2^{-\alpha}$ ribbon synapses. [CF] Histograms of SV distance from membrane [C,D] or PD [E,F] (bin size $5 \mathrm{~nm}$ in C-E and $15 \mathrm{~nm}$ in F) show the number of SVs (right axis) with respect to their distance to the plasma membrane or PD. The cumulative distribution function (solid line) shows the probability of 
SVs (left axis) to be located at a specific distance to the membrane or PD (x-axis). The Kolmogorov-Smirnov test was used to compare the probability distribution of SVs. [C] A large fraction (70\%) of MP-SVs was located within $\sim 25 \mathrm{~nm}$ and a small fraction $(30 \%)$ of SVs was located within $~ 25 \mathrm{~nm}$ to $50 \mathrm{~nm}$ away from the plasma membrane. A significantly different cumulative distribution of SVs was observed between the two genotypes for both the large $(p=0.004)$ and the small $(p=0.02)$ fractions of MP-SVs. [E] The distribution of MP$S V$ s with respect to the PD was unaltered in $R I M-B P 2^{-/}$ribbon synapses. [D] In RIM-BP2 ${ }^{-1}$ ribbon synapses, "outlying"-SVs had a significantly higher probability to be located closer to the membrane ( $p=0.049)$ compared to $R I M-B P 2^{+/+}$ribbon synapses. [F] "Outlying"-SVs had a significantly higher probability to be located more distal from the PD in $R I M-B P 2^{-/}$ribbon synapses ( $p=0.004)$. [A-F] Significance levels: n.s. $p \geq 0.05,{ }^{*} p<0.05,{ }^{* *} p<0.01,{ }^{* *} p<0.001$; $R I M-B P 2^{+/+}$IHCs $(\mathrm{n}=9, \mathrm{~N}=2), R I M-B P 2^{-/}$IHCs $(\mathrm{n}=9, \mathrm{~N}=2), \mathrm{n}=$ number of tomograms, $\mathrm{N}=$ number of mice; age of mice: p21. Panels [C and E] adapted from Krinner et al., 2017.

In the absence of RIM-BP2, "outlying"-SVs were on average positioned further away from the PD and additionally closer to the plasma membrane (Figure 20B, both $p=0.005$ ). The shift in the cumulative distribution of "outlying"-SVs with respect to the plasma membrane indicates that in $R I M-B P 2^{-/} \mathrm{AZs}$, SV are overall shifted into closer proximity to the membrane (Figure 20D, $p=0.049$ ). Whereas the cumulative distribution of "outlying"-SVs with respect to the PD indicates that SV in $R I M-B P 2^{-\alpha} \mathrm{AZs}$, are distributed over a wider presynaptic area (Figure 20F, $p=0.004$ ). Whether the "outlying"-SV pool represents SVs that are pre-synaptically recruited for exocytosis, was not studied yet in IHCs.

\section{RIM-BP2 disruption causes a mild impairment of synaptic sound encoding}

In order to probe the consequences of RIM-BP2 disruption on the auditory systems function, recordings of $A B R s$ and DPOAEs were performed in RIM-BP2-- mice (measurements by Nadine Dietrich). DPOAEs probe the function of OHCs, mediating cochlear amplification. In both genotypes, normal DPOAE amplitudes were recorded (for all F2 intensities: $p>0.05$ ) (Figure 21C), indicating intact mechanoelectrical transduction and cochlear amplification. $A B R s$, on the other hand, reflect the synchronized neural activation of the stages of the auditory pathway. ABR thresholds were mildly but significantly elevated in 8-10 week-old $R I M-B P 2^{-1}$ mice (Figure $21 \mathrm{~A} ; p_{4 \mathrm{kHz}}=0.005, p_{8 \mathrm{kHz}}=0.02, p_{16 \mathrm{kHz}}=0.002$ ). In addition, the ABR wave I - reporting the compound AP of SGNs, which get innervated by IHC ribbon synapses - was significantly reduced (Figure $21 \mathrm{~B} ; p=0.03$ ). This suggests a mild synaptopathic hearing impairment for RIM-BP2-- mice (Moser and Starr, 2016). Interestingly, the ABR wave III amplitude was also significantly reduced (Figure 21B; $p<0.0001$ ) in RIM-BP2mice. The ABR wave III likely represents the synchronized activity of neurons from the superior olivocochlear complex (Melcher et al., 1996), suggesting an additional role of RIMBP2 downstream of the IHC ribbon synapse. 
Moreover, the smaller ABR wave I amplitude in the absence of RIM-BP2 could have different reasons: Changes in the afferent innervation of IHC ribbon synapses or changes in the spiking behavior of the postsynaptic ANFs, both of which were investigated next.
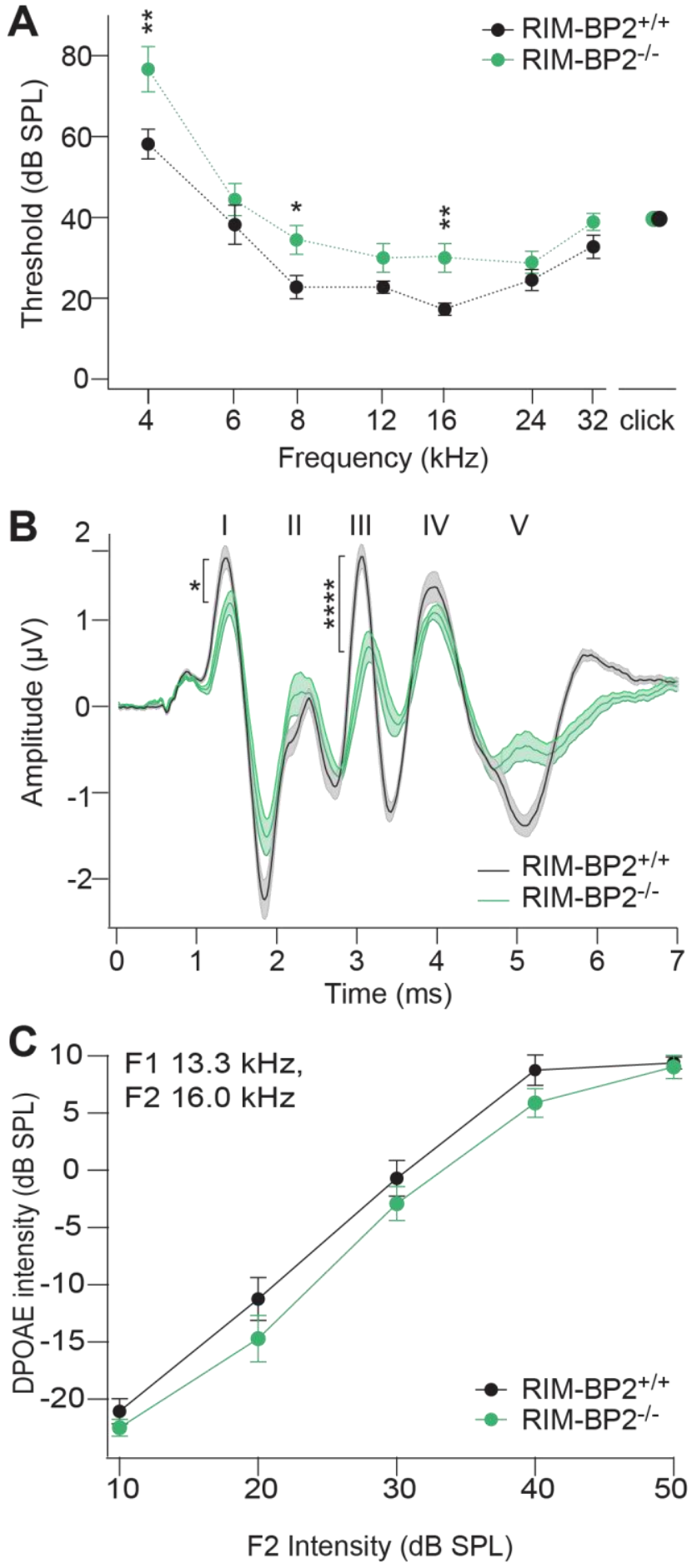

Figure 21: RIM-BP2 disruption causes a mild synaptopathic hearing impairment

[A] ABR thresholds were elevated in RIM-BP2 deficient mice compared to control mice, which reached significance at 4 $(p=0.005), 8(p=0.02)$, and $16 \mathrm{kHz}$ $(p=0.002)$.

[B] Compared to control mice ABR waveforms (80 dB peak equivalent, $20 \mathrm{~Hz}$ stimulation rate) of RIM-BP2 deficient mice elicited a significantly reduced ABR wave I amplitude $(p=0.03)$ and wave III amplitude $(p<0.0001)$, representing the compound AP of SGNs and the olivocochlear nucleus, respectively.

[C] At the frequency of the strongest increase of hearing threshold (16 kHz, see A), otoacoustic emission amplitudes were unaltered in RIM-BP2 deficient mice compared to control mice $(p=0.1$ to 0.9$)$, indicating normal mechanoelectrical transduction (F1 $13.3 \mathrm{kHz}, \mathrm{F} 216$ $\mathrm{kHz}$ ).

[A-C] Data information: Data represent averages, mean \pm SEM; One way ANOVA, $p$-values are from post hoc Tukey's multiple comparison: n.s. $p \geq 0.05,{ }^{*} p<0.05$, ${ }^{* *} p<0.01, \quad{ }^{* * *} p<0.001 ; \quad R I M-B P^{+/+}$ $\mathrm{n}=12$ (black), RIM-BP $/ \mathrm{n}=10$ (green); $n=$ number of mice; age of mice: 8-10 weeks. Panels [A-C] adapted from Krinner et al., 2017. 


\section{RIM-BP2 disruption does not affect IHC afferent connectivity}

First, IHC to afferent fiber connectivity was examined using confocal microscopy and 3D analysis of pre- and postsynaptically immunolabeled synapses. RIBEYE/CtBP2 served as a presynaptic ribbon marker and GluA2/3 served as a postsynaptic marker labeling AMPA receptors (Figure 22A) (Khimich et al., 2005). The number of ribbons (RIBEYE/CtBP2 spots) and ribbon-occupied synapses (number of juxtaposed RIBEYE/CtBP2 and GluA2/3 spots) were comparable in $R I M-B P 2^{-/}$and $R I M-B P 2^{+/+} I H C s$ ( $p=0.9$ and 0.6 , respectively) (Figure 22B), indicating that the afferent IHC connectivity was unaltered.

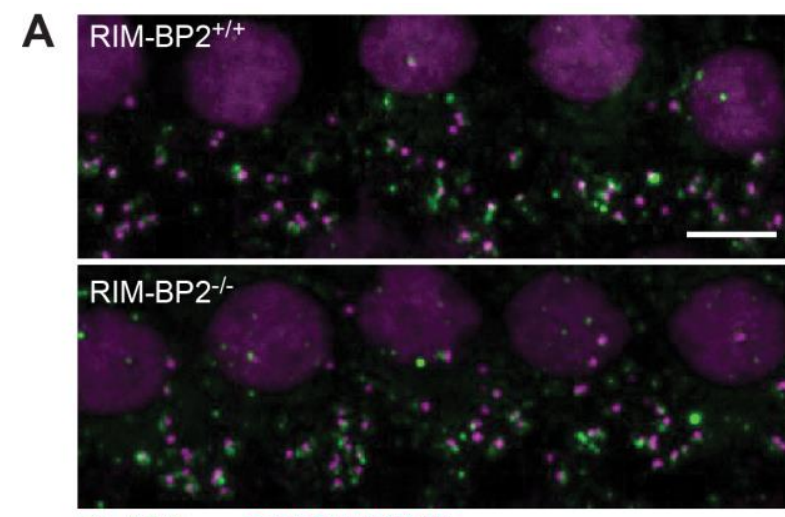

GluA2/3 CtBP2/RIBEYE
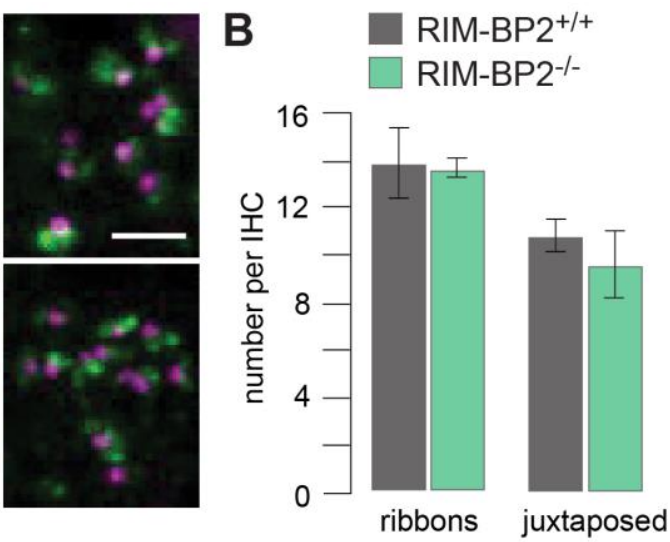

Figure 22: RIM-BP2 loss does not affect IHC afferent connectivity

[A] Left: Maximum projections of confocal stacks from whole mount explants from apical organs of Corti, immunolabeled for CtBP2/RIBEYE (magenta) and GluA2/3 (green). Scale bar: $5 \mu \mathrm{m}$. Right: Higher magnification shows details of synapses. Scale bar: $2 \mu \mathrm{m}$ [B] 3D analysis of fluorescently labeled IHC ribbon synapses shows that the number of ribbons (CtBP2/RIBEYE spots) and the number of ribbon-occupied synapses (number of juxtaposed RIBEYE/CtBP2 and GluA2/3 spots) per IHC were not altered in RIM-BP2 deficient $\left(R I M-B P 2^{-/}, \mathrm{N}=4\right)$ IHCs compared to control $\left(R I M-B P 2^{+/+}, \mathrm{N}=4\right)$ IHCs. Data represent grand averages, mean \pm SEM; Wilcoxon rank test: n.s. $p \geq 0.05,{ }^{*} p<0.05,{ }^{* *} p<0.01$, ${ }^{* * *} p<0.001 ; n=$ number of IHCs, $N=$ number of mice; age of mice: $p 21$. Panels $[A-B]$ adapted from Krinner et al., 2017.

Second, extracellular recordings from postsynaptic ANFs of SGNs were performed to study sound encoding in vivo (experiments and data analysis were performed by Tanvi Butola). This method provides analysis of single AZ activity as each SGN is thought to receive input from just a single IHC AZ (Liberman 1978). Frequency tuning (Figure 23B) and sound thresholds (Figure 23B') were unaltered in SGNs of $R I M-B P 2^{-/}$mice, supporting the findings of intact cochlear amplification. However, a significantly higher fraction of ANFs with low spontaneous firing rates ("low-SR" SGNs) was found in RIM-BP2 deficient mice (Figure 23A, $p=0.04)$. Together, the unaltered sound thresholds and the decreased spontaneous firing rate in $R I M-B P 2^{--}$SGNs could therefore result from the reduction of presynaptic $\mathrm{Ca}^{2+}$ channels (Figure 14 and 15) and a consequential overall reduction of spontaneous SV 
release in IHCs. Likely, the increase in low-SR SGNs is not the consequence of recording from a higher fraction of low-SR / high threshold SGNs in RIM-BP2-- mice, since spike thresholds were unaltered in $R I M-B P 2^{-/}$mice (Figure 32B).

A

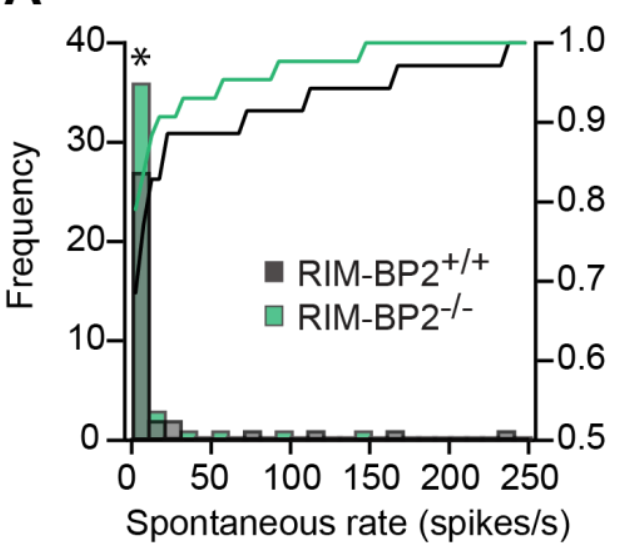

B
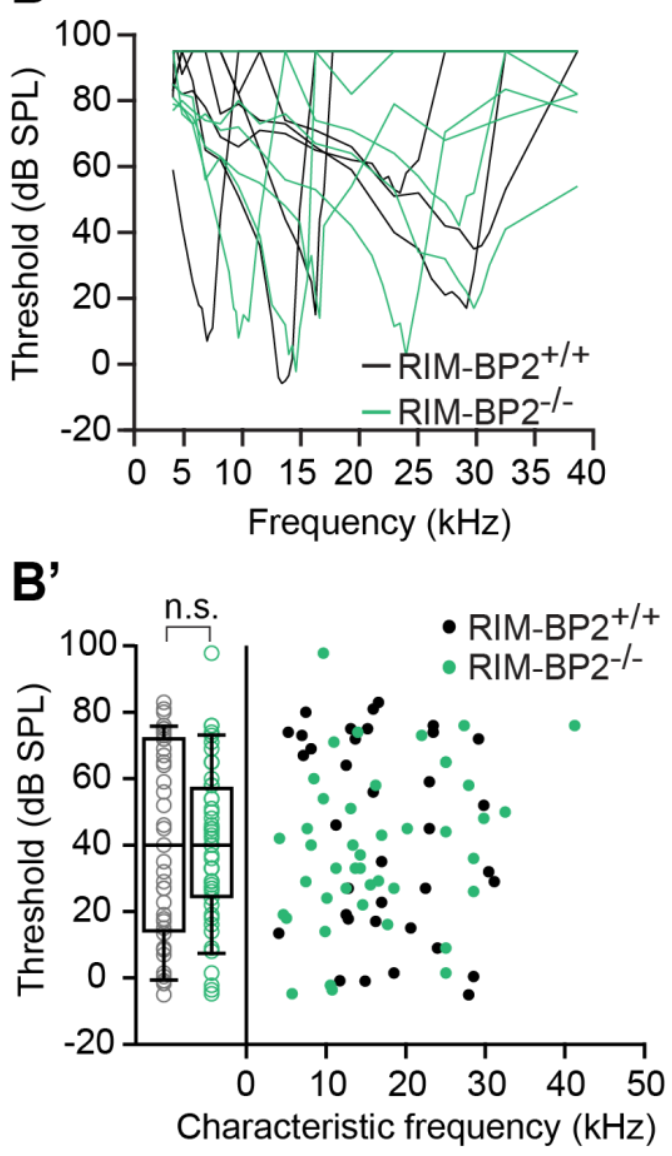

Figure 23: Increased fraction of low-SR SGNs in RIM-BP2/- mice

[A] Distribution of spontaneous firing rates of single units in $R I M-B P 2^{++}$(black; $\mathrm{n}=35 ; \mathrm{N}=6$ ) and $R I M-B P 2^{-/}$(green; $\mathrm{n}=43 ; \mathrm{N}=3$ ) ANFs. The histogram represents the distribution of their frequency (left axis) and the solid lines represent the cumulative probability density (right axis) of spontaneous firing rates. The fraction of ANFs with low spontaneous firing rates was significantly higher in $R I M-B P 2^{-/}$ mice (Wilcoxon rank test, $p=0.04$ ).

[B] Representative tuning curves of ANFs from $R I M-B P 2^{+/+}$(black; $\mathrm{n}=41 ; \mathrm{N}=6$ ) and $R I M$ $B P 2^{--}$(green; $\mathrm{n}=48 ; \mathrm{N}=4$ ) mice demonstrate clear and unimpaired frequency tuning in RIM-BP2/- SGNs.

[B'] ANF sound thresholds at their characteristic frequency were comparable between the two genotypes (Wilcoxon rank test, $p=0.82$ ): $40.75 \pm 4.60$ (median: 40.0 ) dB for $R I M-B P 2^{+++}(\mathrm{n}=41 ; \mathrm{N}=6)$ and $39.66 \pm 3.4$ (median: 40.00) dB for RIM-BP2 ${ }^{-/}(\mathrm{n}=48$; $\mathrm{N}=4$ ). Data reported as mean $\pm \mathrm{SEM}$. Each data point represents the response of a single ANF.

[A-B'] Tanvi Butola performed experiments and data analysis. Data information: Significance levels: n.s. $p \geq 0.05,{ }^{*} p<0.05$, ${ }^{* *} p<0.01,{ }^{* * *} p<0.001$; Box and whisker plot represents median, lower/upper quartiles and $10^{\text {th }}-90^{\text {th }}$ percentiles; $n=$ number of ANFs, $\mathrm{N}=$ number of animals. Age of mice: 10 weeks. Panels [A-B'] adapted from Krinner et al., 2017. 
The onset firing rate (peak rate) of SGN responses (Figure 24A) evoked by 50 ms tone bursts, at their characteristic frequency and $30 \mathrm{~dB}$ SPL above spike threshold, reflects the maximal initial RRP SV release rates of the presynaptic IHCs. The adapted spike rate reflects RRP depletion and replenishment dynamics convolved with postsynaptic refractoriness (Figure 24A) (Buran et al., 2010; Frank et al., 2010; Wittig and Parsons, 2008). The adapted rate was unaltered in $R I M-B P 2^{-}$SGNs, whereas the peak firing rate was significantly reduced (Figure 24A, $p=0.02$ ). Furthermore, the firing onset was significantly delayed in $R I M-B P 2^{-/} S G N s$, which is reflected by an increased median first spike latency (FSL) (Figure 24A', $p=0.004$ ). On the other hand, the FSL variance remained unchanged in RIM-BP2-/ SGNs (Figure 24A'). Likely, the mild impairment of sound onset coding reflected by the reduced peak firing rate and delayed firing onset explain the $A B R$ wave I amplitude reduction and the increased hearing thresholds in $R I M-B P 2^{-/}$mice.

The normal adapted spike rate of $R I M-B P 2^{-}$SGNs (Figure 24A) does not support the notion from IHC electrophysiology, where a reduced rate for the sustained phase of exocytosis (Figure 16A) and slower SV recruitment after RRP depletion (Figure 18B) was found in RIM$B P 2^{-1} \mathrm{IHCs}$. To investigate this in more detail in vivo, the forward masking paradigm (Harris and Dallos, 1979) was used to measure SV-replenishment and provide an estimate of the kinetics of the RRP recovery after partial depletion (Figure 24B). In $R I M-B P 2^{-}$SGNs, probe responses following a $100 \mathrm{~ms}$ masker tone and short recovery intervals tended to be smaller for $4 \mathrm{~ms}$ inter-stimulus-intervals ( $p=0.07)$ and were significantly reduced for $16 \mathrm{~ms}$ interstimulus-intervals $(p=0.04)$. For longer inter-stimulus-intervals, the recovered response in RIM-BP2/- SGNs was comparable to that of $R I M-B P 2^{++}$SGNs and the recovery time constant was unaltered. In summary, these data point towards slower recovery from forward masking, which agrees well with the notion from IHC electrophysiology, suggesting that without RIM-BP2, fast SV-replenishment is slowed. 

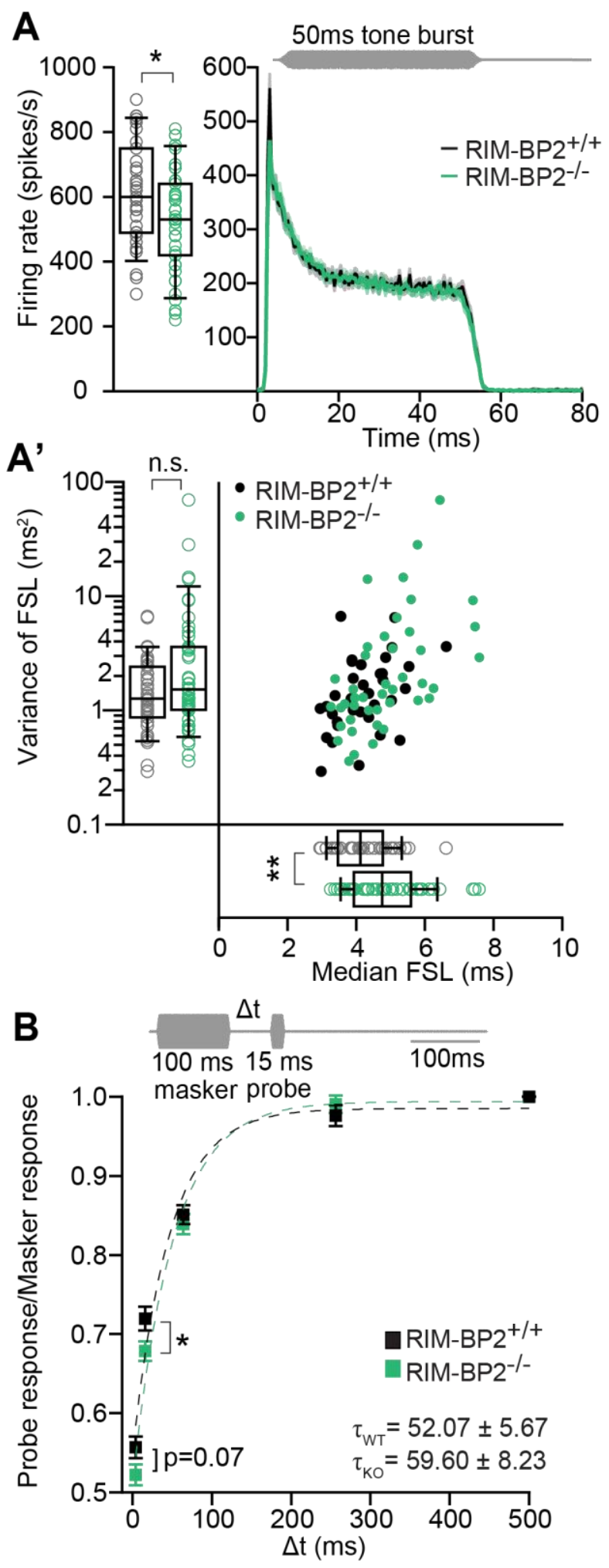

Figure 24: Reduced onset-firing rate, increased first spike latency and slower recovery in RIM-BP2-/ SGNs

[A] Peak-aligned peri-stimulus time histogram (PSTH) of the ANF responses to $50 \mathrm{~ms}$ tone burst stimulation (at characteristic frequency, $30 \mathrm{~dB}$ above threshold; stimulus illustrated in gray) in $R I M-B P 2^{+/+}$(black; $\mathrm{n}=35 ; \mathrm{N}=6$ ) and $R I M-$ $\mathrm{BP2}^{-1-}$ (green; KO $\mathrm{n}=43 ; \mathrm{N}=4$ ). $\mathrm{PSTH}$ presented as mean (solid lines) \pm SEM (shaded area). Peak onset firing rate (left panel) was reduced in RIM-BP2-- ANFs (Student's t-test, $p=0.02$ ).

[A'] Median first spike latency (FSL) of PSTH (same ANF single units as in A) was increased in RIM-BP2- ANFs (Student's t-test, $p=0.004$ ), while the variance of FSL remained unchanged (Wilcoxon rank test, $p=0.23$ ).

[B] The time course of recovery of the ANF was assessed by a 100 ms masker tone followed by a $15 \mathrm{~ms}$ probe tone presented after a silent interval (recovery time) of variable duration $(\Delta \mathrm{t})$ (at characteristic/best frequency, $30 \mathrm{~dB}$ threshold; stimulus paradigm illustrated in gray). Recovery was plotted as the ratio of ANF peak probe tone response to ANF peak masker tone response (solid boxes \pm SEM) and was reduced in $R I M-B P 2^{-/}$ ANFs for short recovery times ( $\Delta \mathrm{t} 16 \mathrm{~ms}$ : Student's t-test, $p=0.04)$. Dotted lines represent single exponential fits to the time course of recovery. Time constants ( $\mathrm{T}$ ) of recovery (mean \pm SEM) were comparable between the two genotypes (Wilcoxon rank test, $p=0.81$ ). $R I M-B P 2^{+/+}$ (black; $\mathrm{n}=40 ; \mathrm{N}=6$ ) and $R I M-B P 2^{-/}$ (green; KO $n=43 ; \mathrm{N}=4$ ).

[A-B] Tanvi Butola performed experiments and data analysis. Data information: Significance levels: n.s. $p \geq$ $0.05,{ }^{*} p<0.05,{ }^{* *} p<0.01,{ }^{* * *} p<0.001$; Box and whisker plots represent median, lower/upper quartiles and $10^{\text {th }}-90^{\text {th }}$ percentiles; $n=$ number of ANFs, $N=$ number of animals. Age of mice: 10 weeks. Panels [A-B] adapted from Krinner et al., 2017. 


\subsection{Discussion}

Faithful encoding of acoustic information at the IHC-SGN synapse requires precise conversion of a sound-driven IHC receptor potential to presynaptic glutamate release. To achieve this challenging task, voltage-gated L-type (Cav1.3) $\mathrm{Ca}^{2+}$-channels are positioned within nanometer proximity to the synaptic vesicle fusion machinery (Pangršič et al., 2015). At AZs of mature apical mouse IHCs approximately 80-100 $\mathrm{Ca}^{2+}$-channels are thought to be clustered in the plasma membrane below the ribbon (Brandt et al., 2005; Wong et al., 2014). Direct electrophysiological access to IHCs enables the examination of depolarization-induced presynaptic $\mathrm{Ca}^{2+}$-influx and simultaneous analysis of individual $\mathrm{AZ}$ $\mathrm{Ca}^{2+}$-influx via fast confocal $\mathrm{Ca}^{2+}$-imaging. Therefore, the $\mathrm{IHC}$ ribbon-type synapse is a suitable model system for detailed analysis of $\mathrm{Ca}^{2+}$-channel abundance and localization as well as $\mathrm{Ca}^{2+}$-influx - exocytosis coupling.

So far, only few cytomatrix proteins have been identified that regulate the number, function and coupling of $\mathrm{Ca}^{2+}$-channels to the IHC vesicular release machinery. In my thesis, I examined the role of the candidate molecular linker RIM-BP2 in murine IHCs - by analyzing the effects of its genetic disruption on the structure and function of IHC ribbon synapses. Confocal and STED microscopy indicated that RIM-BP2 is present at the IHC AZs as part of the typically stripe-like presynaptic density at IHC AZs (Figure 12). There, it appears to promote (1) the abundance of synaptic $\mathrm{Ca}^{2+}$-channels and, in addition, (2) fast SVreplenishment. In support of (1), STED microscopy of Cav1.3 immunofluorescence (Figure 15), live-cell confocal $\mathrm{Ca}^{2+}$-imaging of individual AZs (Figure 14), and whole-cell patch clamp recordings (Figure 13) collectively demonstrated a reduced number of Cav1.3 $\mathrm{Ca}^{2+}$ channels at the AZs of RIM-BP2 deficient IHCs. In support of (2), in vitro and in vivo electrophysiology showed significantly reduced RRP exocytosis during short but not long inter-stimulus-intervals and reduced sustained phase of exocytosis indicative of slowed RRP recovery (Figures 16, 18 and 24). Further, morphological changes in the spatial organization of MP-SVs with respect to the plasma membrane were found by electron tomography (Figure 20). RIM-BP2 deficiency however did not alter $\mathrm{Ca}^{2+}$-nanodomain-like control of exocytosis (Figure 17) in IHCs. Finally, RIM-BP2 deletion caused a mild impairment of hearing (Figure 21) and sound onset coding in SGNs (Figure 24) that likely reflects the presynaptic impairment of $\mathrm{Ca}^{2+}$ signaling and SV-replenishment. In summary, RIM-BP2 was identified as a central part of the cytomatrix of IHC AZs promoting the number of synaptic L-type $\mathrm{Ca}^{2+}$-channels and fast RRP-replenishment. 


\section{Impact of RIM-BP2 on synaptic Ca ${ }^{2+-}$-channel abundance and their coupling to SVs}

As found in previous studies for RIM2a (Jung et al., 2015a) and bassoon (Frank et al., 2010), RIM-BP2 promotes the synaptic abundance of voltage-gated L-type $\mathrm{Ca}^{2+}$-channels at the IHC AZs. Interestingly, such function for RIM-BP2 was also reported for the Drosophila NMJ (Liu et al., 2011; Müller et al., 2015), but not for any conventional mammalian synapse (Grauel et al. 2016; Acuna et al. 2015).

\section{RIM-BP2 regulates the abundance of L-type $\mathrm{Ca}^{2+}$-channels at ribbon synapses}

Evidence that RIM-BP2 promotes the synaptic abundance of voltage-gated L-type $\mathrm{Ca}^{2+}$ channels at the IHC AZs is based on IHC electrophysiology, synaptic confocal $\mathrm{Ca}^{2+}$-imaging and super-resolution STED microscopy of Cav1.3 immunofluorescence. $\mathrm{Ca}^{2+}$-imaging of single AZs revealed a $40 \%$ reduction in presynaptic $\mathrm{Ca}^{2+}$-influx (Figure 14) in RIM-BP2 deficient IHCs. The $51 \%$ decrease found in the semi-quantitative Cav1.3 immunofluorescence image analysis further argued for a reduction in synaptic $\mathrm{Ca}^{2+}$ channels. Moreover, the nanoscale structure of $\mathrm{Cav}_{v} 1.3 \mathrm{Ca}^{2+}$-channel clusters revealed a significant shortening of the long cluster axis' FWHM (16\% shorter) (Figure 15) upon RIMBP2 disruption. Furthermore, the whole-cell $\mathrm{Ca}^{2+}$-current was significantly reduced (by 20\%) (Figure 13 and 16), but Cav1.3 $\mathrm{Ca}^{2+}$-channel gating and inactivation were unaltered (Figure 13). The latter further supports the notion of a reduction in $\mathrm{Ca}^{2+}$-channel numbers in the absence of RIM-BP2. All in all, the reduction in synaptic $\mathrm{Ca}^{2+}$-channels $(40 \%)$ exceeded the reduction in the whole-cell $\mathrm{Ca}^{2+}$-current $(20 \%)$, indicating that in addition to a reduction in the number of $\mathrm{Ca}^{2+}$-channels at the $\mathrm{AZ}$, potentially a higher fraction of extrasynaptic $\mathrm{Ca}^{2+}$ channels (Brandt et al., 2005) might be present in RIM-BP2-/ IHCs.

The possibly higher fraction of extrasynaptic $\mathrm{Ca}^{2+}$-channels in $\mathrm{RIM-BP2^{-/ }}$ IHCs might point towards delayed synapse maturation (Wong et al., 2014; Zampini et al., 2010) upon RIMBP2 disruption. This would go along with reduced $\mathrm{Ca}^{2+}$-channel open probability (Zampini et al., 2010), which could also contribute to the $20 \%$ reduced $\mathrm{Ca}^{2+}$-current, and $40 \%$ reduced synaptic $\mathrm{Ca}^{2+}$-influx in $\mathrm{RIM}-\mathrm{BP}^{-/} \mathrm{IHCs}$. Immature IHC synapses were previously found to exhibit (1) weak $\mathrm{Ca}^{2+}$-channel inactivation and low $\mathrm{Ca}^{2+}$-channel open probability (Zampini et al., 2010, 2013), (2) non-linear apparent $\mathrm{Ca}^{2+}$-dependence of exocytosis, indicating a loose coupling between $\mathrm{Ca}^{2+}$-influx and exocytosis (Wong et al., 2014), (3) AZs containing several small ribbons (Sobkowicz et al., 1982; Wong et al., 2014) and (4) prominent appearance of extrasynaptic $\mathrm{Ca}^{2+}$-channels that formed small and round clusters (Wong et al., 2014; Zampini et al., 2010). Based on these observations, the following findings argue against a developmental deficit in $R I M-B P^{--} I H C$ AZs: (1) The $\mathrm{Ca}^{2+}$-current inactivation 
kinetics were unaltered in $R I M-B P 2^{-/} I H C s$ (Figure 13). (2) The apparent $\mathrm{Ca}^{2+}$-dependence of exocytosis was quasi-linear in $\mathrm{RIM-BP2^{-/ }}$ IHCs indicating tight nanodomain-like $\mathrm{Ca}^{2+}$ exocytosis coupling (Figure 17). (3) Both, the ultrastructural and immunohistochemical analysis revealed unaltered number and size of synaptic ribbons in $R I M-B P 2^{-/} \mathrm{IHCs}$ when compared to $R I M-B P^{+/+}$littermates (Figure 19 and 22). (4) The majority of $\mathrm{Ca}^{2+}$-channel clusters in $R I M-B P 2^{-1} A Z s$ still appeared in a "stripe- or double stripe-like" arrangement (62\% in $R I M-B P 2^{--}$AZs compared to $66 \%$ in $R I M-B P^{++} A Z s$ ) and co-localized with synaptic CtBP2/RIBEYE marked synaptic ribbons (Figure 15). Hence, apart from the possibly higher fraction of extrasynaptic $\mathrm{Ca}^{2+}$-channels in $\mathrm{RIM}-\mathrm{BP}^{-/} \mathrm{IHCs}$, these data mainly argue against delayed synapse maturation in RIM-BP2 deficient IHC.

Even though a reduction in the number of synaptic $\mathrm{Ca}^{2+}$-channels upon RIM-BP2 disruption was not reported for conventional mammalian synapses (Grauel et al. 2016; Acuna et al. 2015), it should be noted that my findings are consistent with recent work from the murine rod bipolar cell ribbon synapse (Luo et al., 2017). In this study, the authors reported a role of RIM-BP2 in tethering L-type (Cav1.3) $\mathrm{Ca}^{2+}$-channels to ribbon synapse AZs (Luo et al., 2017), thereby implicating a common function of RIM-BPs at mammalian ribbon synapses. Moreover, in the latter study, synaptic Cav1.3 immunofluorescence was significantly reduced in $R I M-B P 1 / 2$ double-knockout rod bipolar cell ribbon synapses and presynaptic $\mathrm{Ca}^{2+}$-current peak amplitude was significantly reduced in $R I M-B P 1 / 2$ double-knockout (50\%) and RIM-BP1 and RIM-BP2 single-knockout rod bipolar cells (20-30\%) (Luo et al., 2017).

Why are the effects of RIM-BP disruption on the $\mathrm{Ca}^{2+}$-channels so diverse among different synapse types? The specific AZ ultrastructure, the expression of specific proteins and/or protein isoforms, the level of their expression as well as genetic redundancy might fine-tune synapses for their needs and thereby drive the specialization of a given synapse type. While ribbon-type synapses express mainly L-type $\mathrm{Ca}^{2+}$-channels (Brandt et al., 2003; Morgans, 2001; Platzer et al., 2000), in the hippocampal synapses $\mathrm{N}$ - and $\mathrm{P} / \mathrm{Q}$-type $\mathrm{Ca}^{2+}$-channels are mainly found (Luebke et al., 1993; Takahashi and Momiyama, 1993; Wheeler et al., 1994; Wu et al., 1999). Further differences may appear upon synapse development. For example, whereas immature Calyx of Held synapses predominantly express $\mathrm{N}$-type $\mathrm{Ca}^{2+}$ channel types, mature synapses predominantly operate via $\mathrm{P} / \mathrm{Q}$-type $\mathrm{Ca}^{2+}$-channels (Fedchyshyn, 2005; Scholz and Miller, 1995). 


\section{Potential impact of RIM-BP2 - bassoon interaction}

A selective recruitment of $\mathrm{P} / \mathrm{Q}$-type $\mathrm{Ca}^{2+}$-channels by bassoon was previously found in hippocampal synapse AZs in a bassoon knockout study (Davydova et al., 2014). Interestingly, the authors further report that bassoon requires the interaction with RIM-BPs for the recruitment of $P / Q$-type $\mathrm{Ca}^{2+}$-channels to synapses (Davydova et al., 2014). While bassoon disruption caused significantly reduced synaptic RIM-BP2 and P/Q-type $\mathrm{Ca}^{2+}$ channel levels, also interfering with the bassoon - RIM-BP2 interaction decreased the number of P/Q-type channels at the release sites (Davydova et al., 2014). Hence, the authors concluded that RIM-BPs might form a molecular hub in a bassoon - RIM-BP - $\mathrm{Ca}^{2+}$ channel tripartite complex, as RIM-BPs can simultaneously bind to $\mathrm{Ca}^{2+}$-channels and the presynaptic cytomatrix via bassoon (Davydova et al., 2014). Thereby, RIM-BP - bassoon interaction is likely mediated between RIM-BP C-terminal SH3I domain and the PXXP motif of bassoon (Davydova et al., 2014), whereas RIM-BP - $\mathrm{Ca}^{2+}$-channel interaction appears to involve the N-terminal SH3III domain of RIM-BPs and the PXXP motif in the C-terminal region of the Cava subunit of the $\mathrm{Ca}^{2+}$-channel (Hibino et al., 2002).

Interestingly, the same study reported that there is no evidence for direct bassoon interaction with L-type $\mathrm{Ca}^{2+}$-channels (Davydova et al., 2014), consistent with (Frank et al., 2010). Disruption of bassoon in IHCs - that mainly express L-type $\mathrm{Ca}^{2+}$-channels (Brandt et al., 2003; Platzer et al., 2000) - revealed a strong ( 60\%) reduction in synaptic $\mathrm{Ca}^{2+}$ influx in bassoon mutant IHCs (Frank et al., 2010), indicating a role of bassoon in regulating the abundance of synaptic L-type $\mathrm{Ca}^{2+}$-channels. Since however RIM-BPs, but not bassoon could be shown to interact biochemically with L-type $\mathrm{Ca}^{2+}$-channels (Davydova et al., 2014; Hibino et al., 2002, Frank et al., 2010), the reduction of synaptic L-type $\mathrm{Ca}^{2+}$-channels upon bassoon disruption in IHCs (Frank et al., 2010) may be mediated by bassoon - RIM-BP2 interaction. Nonetheless, the reduction in synaptic $\mathrm{Ca}^{2+}$-influx in bassoon mutant AZs (60\%) exceeded the reduction in synaptic $\mathrm{Ca}^{2+}$-influx reported in the absence of RIM-BP2 (40\%) (Figure 14), suggesting that the reduction of synaptic $\mathrm{Ca}^{2+}$-channels in the absence of bassoon might only be partially based on the potential bassoon - RIM-BP2 interaction. On the other hand, bassoon disruption was associated with ribbon detachment from the plasma membrane (Frank et al., 2010; Khimich et al., 2005), which could give rise to secondary effects contributing to the reduction in the number of synaptic $\mathrm{Ca}^{2+}$-channels. A good validation of this hypothesis would be to test if synaptic RIM-BP2 expression levels were reduced in bassoon mutant IHC AZs. In RIM-BP2 deficient IHC AZs, bassoon cluster shapes and sizes appeared normal when measured by STED microscopy and subsequent 2D Gaussian fitting of synaptic bassoon immunofluorescence (Figure 15). Surprisingly, upon RIM-BP2 disruption, a statistically significant $~ 20 \%$ increase in bassoon fluorescence intensity integrals was observed (Figure 15). This suggests first, that bassoon might have 
been up-regulated by a compensatory mechanism counteracting the loss of RIM-BP2 and second, that bassoon - RIM-BP2 interaction might be relevant not only for the abundance of $\mathrm{P} / \mathrm{Q}$-type $\mathrm{Ca}^{2+}$-channels in hippocampal synapse AZs (Davydova et al., 2014), but also for L-type $\mathrm{Ca}^{2+}$-channels at IHC ribbon-type synapse AZs.

\section{Potential compensatory recruitment of $\mathrm{N}$-type $\mathrm{Ca}^{2+}$-channels upon loss of RIM-BP2}

Besides the finding, that bassoon requires the interaction with RIM-BP2 for the recruitment of P/Q-type $\mathrm{Ca}^{2+}$-channels to synapses (Davydova et al., 2014), the authors also found a higher fraction of $\mathrm{N}$-type $\mathrm{Ca}^{2+}$-channels at bassoon deficient hippocampal neuron $\mathrm{AZs}$, which compensated total $\mathrm{Ca}^{2+}$-channel numbers (Davydova et al., 2014). Hence, one would expect an equal reduction in the synaptic P/Q-type $\mathrm{Ca}^{2+}$-channel number and possibly compensational recruitment of $\mathrm{N}$-type $\mathrm{Ca}^{2+}$-channels upon RIM-BP2 disruption. Whether this hypothesis is true, was only partly addressed in a RIM-BP2 knockout study in hippocampal neurons (Grauel et al., 2016). In this study, super-resolution microscopy revealed a change in the relative distribution of $\mathrm{P} / \mathrm{Q}$-type $\mathrm{Ca}^{2+}$-channels with respect to the AZ scaffold and a localization of P/Q-type $\mathrm{Ca}^{2+}$-channels more distal from the $\mathrm{AZ}$ when RIMBP2 is absent (Grauel et al., 2016). However, changes in synaptic P/Q-type and N-type $\mathrm{Ca}^{2+}$-channel levels were not measured in the immunofluorescence cluster analysis in RIMBP2 deficient hippocampal neurons (Grauel et al., 2016). Moreover, SynGCamp6f $\mathrm{Ca}^{2+}$ imaging was performed to estimate synaptic $\mathrm{Ca}^{2+}$-channel influx (Grauel et al., 2016). However, there, no distinction of $\mathrm{Ca}^{2+}$-influx through different $\mathrm{Ca}^{2+}$-channel types was made, meaning that the summated $\mathrm{Ca}^{2+}$-influx through all $\mathrm{Ca}^{2+}$-channel types was measured. By applying the pharmacological $\mathrm{N}$-type $\mathrm{Ca}^{2+}$-channel blocker $\omega$-conotoxin GVIA, or P/Q-type $\mathrm{Ca}^{2+}$-channel blocker $\omega$-agatoxin IVA during synaptic SynGCamp6f $\mathrm{Ca}^{2+}$-imaging, one could reach more precise estimates on the contribution of synaptic P/Q-type and N-type $\mathrm{Ca}^{2+-}$-channels in RIM-BP2 deficient hippocampus neurons. Therefore, one could speculate that RIM-BP2 might have a potential function in regulating the abundance of P/Q-type $\mathrm{Ca}^{2+}$ channels in hippocampal synapse AZs.

Further indication that recruitment of $\mathrm{N}$-type $\mathrm{Ca}^{2+}$-channels might have compensated for potential P/Q-type $\mathrm{Ca}^{2+}$-channel loss upon RIM-BP2 disruption in hippocampal neurons is the increased coupling distance between $\mathrm{Ca}^{2+}$-channels and SVs in RIM-BP2 deficient neurons (Grauel et al., 2016). In both hippocampal neurons and the calyx of Held, a correlation between nanodomain-like and microdomain-like coupling and the presence of P/Q-type, and N-type $\mathrm{Ca}^{2+}$-channels, respectively, was found (Fedchyshyn, 2005, Goswami et al., 2012). Hence, such looser $\mathrm{Ca}^{2+}$-influx - exocytosis coupling in RIM-BP2 deficient hippocampal neurons could be caused by the compensatory recruitment of $\mathrm{N}$-type $\mathrm{Ca}^{2+}-$ channels to $\mathrm{AZs}$ upon synaptic $\mathrm{P} / \mathrm{Q}$-type $\mathrm{Ca}^{2+}$-channel loss. 
A reduction in synaptic $\mathrm{P} / \mathrm{Q}$-type $\mathrm{Ca}^{2+}$-channel number and possibly compensational recruitment of $\mathrm{N}$-type $\mathrm{Ca}^{2+}$ channels upon $\mathrm{RIM}-\mathrm{BP} 2$ disruption is also conceivable for the calyx of Held synapse. However, consistent with work from hippocampal neurons (Grauel et al., 2016), the RIM-BP1/2 knockout study at the calyx of Held synapse reported no reduction of presynaptic $\mathrm{Ca}^{2+}$-currents upon RIM-BP1/2 disruption, when investigated by electrophysiology (Acuna et al. 2015). Further, the authors used the selective P/Q-type $\mathrm{Ca}^{2+}$-channel blocker $\omega$-agatoxin IVA and found no change in the relative contribution of different $\mathrm{Ca}^{2+}$-channel subtypes to the total presynaptic $\mathrm{Ca}^{2+}$-current upon RIM-BP1/2 disruption (Acuna et al. 2015). Nonetheless, whole-cell recordings of presynaptic $\mathrm{Ca}^{2+}$-influx sum synaptic and extrasynaptic $\mathrm{Ca}^{2+}$-influx and thereby do not provide insight into the different $\mathrm{Ca}^{2+}$-channel subtypes that mediate exocytosis at the AZs. This insight could be however gained by direct examination of synaptic transmission in the presence of either the pharmacological $\mathrm{N}$-type $\mathrm{Ca}^{2+}$-channel blocker $\omega$-conotoxin $\mathrm{GVIA}$, or $\mathrm{P} / \mathrm{Q}$-type $\mathrm{Ca}^{2+}$-channel blocker $\omega$-agatoxin IVA in RIM-BP2 deficient calyxes. In the above mentioned bassoon knockout study, this experiment lead to one of the key findings, namely the recovery of evoked synaptic transmission through compensatory recruitment of $\mathrm{N}$-type $\mathrm{Ca}^{2+}$-channels to AZs upon bassoon disruption (Davydova et al., 2014). While application of the N-type $\mathrm{Ca}^{2+}$-channel blocker $\omega$-conotoxin GVIA significantly reduced evoked excitatory postsynaptic currents (eEPSCs) in bassoon deficient neurons compared to wildtype neurons, the $\mathrm{P} / \mathrm{Q}$-type $\mathrm{Ca}^{2+}$-channel blocker $\omega$-agatoxin IVA had no effect (Davydova et al., 2014). This indicates a switch from mainly $P / Q$-type $\mathrm{Ca}^{2+}$-channel mediated synaptic transmission in wildtype neurons to $\mathrm{N}$-type $\mathrm{Ca}^{2+}$-channel mediated synaptic transmission in bassoon deficient neurons (Davydova et al., 2014). Therefore, this experiment could also shed light on the relative contribution of different $\mathrm{Ca}^{2+}$-channel subtypes to synaptic transmission in RIM-BP1/2 deficient calyxes. Hence, as mentioned above, one could therefore speculate that RIM-BP1/2 might a potential function in regulating the abundance of $P / Q$-type $\mathrm{Ca}^{2+}$-channels also at $A Z$ s of calyx of Held synapses.

If loss of synaptic $\mathrm{P} / \mathrm{Q}$-type $\mathrm{Ca}^{2+}$-channels and compensatory recruitment of $\mathrm{N}$-type $\mathrm{Ca}^{2+}$ channels to AZs upon RIM-BP2 disruption would be true, one could further argue that RIMBP1/2 themselves do not directly mediate the tight coupling of SVs and $\mathrm{Ca}^{2+}$-channels, which was reported for RIM-BP1/2 deficient calyx of Held synapses (Acuna et al. 2015). It would be rather the consequence of the larger contribution of $\mathrm{N}$-type $\mathrm{Ca}^{2+}$-channels, which per se are characterized by a looser microdomain-like $\mathrm{Ca}^{2+}$-channel - SV coupling (Eggermann et al., 2011; Fedchyshyn, 2005; Goswami et al., 2012). 


\section{Tight $\mathrm{Ca}^{2+}$ nanodomain-like coupling at IHC ribbon synapses}

In this work, the $\mathrm{Ca}^{2+}$-channel - SV coupling in IHCs was addressed by testing the apparent $\mathrm{Ca}^{2+}$ cooperativity $m$ of RRP exocytosis in RIM-BP2 deficient IHCs, however no indication for such looser coupling was found (Figure 17). Previously, modeling and biophysical experiments, in which either the number of open channels $\left(N_{C a} \times p_{o p e n}\right)$ or single channel current $\left(i_{C a}\right)$ was manipulated, carefully addressed $\mathrm{Ca}^{2+}$-exocytosis coupling (Wong et al., 2014). For a $\mathrm{Ca}^{2+}$ microdomain-like control of exocytosis, the apparent $\mathrm{Ca}^{2+}$ cooperativity $m$ was expected to be similar to the intrinsic $\mathrm{Ca}^{2+}$ cooperativity (4-5) for both types of manipulation (Beutner et al., 2001). For a $\mathrm{Ca}^{2+}$ nanodomain-like control, on the other hand, a $m$ close to unity was expected and indeed observed during changes of the number of open channels (Brandt et al., 2005; Wong et al., 2014). Indeed, mature IHCs showed nonlinear $\mathrm{Ca}^{2+}$ dependence when manipulating $i_{C a}\left(m\right.$ around 3 ), but nearly linear $\mathrm{Ca}^{2+}$ cooperativity $m$ of RRP exocytosis when manipulating $N_{C a} X p_{o p e n}$ by applying a successive dihydropyridine block of L-type $\mathrm{Ca}^{2+}$ channels ( $m$ around 1.5) (Wong et al., 2014). Here, for RIM-BP2 deficient IHCs $m$ was also found to be 1.5, arguing against a disruption of $\mathrm{Ca}^{2+}$ nanodomain-like control of exocytosis in the absence of RIM-BP2. Other molecular linkers, potentially compensating for RIM-BP2-deficiency, might mediate the molecular coupling of $\mathrm{Ca}^{2+}$-channels and vesicular $\mathrm{Ca}^{2+}$-sensors in IHCs. These findings are suggestive of a highly and tightly regulated release machinery at IHC AZs that serves the demands of high SV turnover with precise timing. If combinatory deletion of several proteins like, for example, RIM and RIM-BP2 would elicit any measurable effect will have to be examined in future experiments. Interestingly, in retinal ribbon synapses, such looser coupling of $\mathrm{Ca}^{2+}$ channels to SVs was observed upon RIM-BP1/2 deletion (Luo et al., 2017), indicating that even though synapses of high similarity (e.g. presence of synaptic ribbon and expression of L-type $\mathrm{Ca}^{2+}$-channels) employ different strategies to fine-tune synaptic transmission. This is further reflected in a differential expression of presynaptic proteins, e.g. lack of neuronal SNAREs (Nouvian et al., 2011) and presence of otoferlin (Pangršič et al., 2012) in IHCs.

\section{Potential interaction partners of RIM-BP2 at IHCs}

Besides interaction of RIM-BP2 with L-type $\mathrm{Ca}^{2+}$-channels (Hibino et al., 2002) and bassoon (Davydova et al., 2014), also other CAZ proteins might interact with RIM-BP2 at IHC AZs and thereby shape and fine-tune synaptic transmission of IHCs.

\section{Synergistic interplay of RIM-BP2 and RIMs}

RIM2 $\alpha$ is an intensively studied synaptic cytomatrix protein and its genetic deletion caused a reduction in synaptic $\mathrm{Ca}^{2+}$-channel number and presynaptic $\mathrm{Ca}^{2+}$-influx ( 36\%) at $\mathrm{IHC}$ 
AZs (Jung et al., 2015a). Interestingly, disruption of RIM2 $\alpha$ did also not lead to an obvious switch to $\mathrm{Ca}^{2+}$ microdomain-like control of exocytosis at IHC synapses (Jung et al., 2015a). RIM2 $\alpha$ is thought to bind IHC L-type channels through C-terminal interaction with the auxiliary Cavß channel subunits and through interaction with the C-terminus of the Cava subunit (Gebhart et al., 2010; Kiyonaka et al., 2007). However, the latter direct RIM2 interaction with the pore-forming a subunit of L-type $\mathrm{Ca}^{2+}$-channels is still under debate (Coppola et al., 2001; Kaeser et al., 2011; Picher et al., 2017). Interestingly, apart from the direct interaction of RIMs with $\mathrm{Ca}^{2+}$-channels, several studies pointed out the importance of an indirect protein interaction between RIMs and $\mathrm{Ca}^{2+}$-channels through RIM-BPs in a tripartite complex (Hibino et al., 2002; Kaeser et al., 2011). The importance of the tripartite complex formation was for example shown for tethering of $\mathrm{N}$ - and $\mathrm{P} / \mathrm{Q}$-type $\mathrm{Ca}^{2+}$-channels to the AZ (Kaeser et al., 2011). In this study, rescue experiments in RIM deficient neurons with a RIM peptide containing the PDZ domain and the PXXP motif - the RIM-BP binding sequence - restored normal $\mathrm{Ca}^{2+}$-dependence of exocytosis, whereas loss of the RIM-BPbinding sequences blocked rescue of $\mathrm{Ca}^{2+}$ influx in RIM deficient neurons (Kaeser et al., 2011). Along these lines, expression of RIM2 and the Cava subunit of L-type channels alone did not lead to co-localization of both proteins in a heterologous expression system, whereas additional expression of RIM-BP2 induced co-localization of all three proteins and enhanced exocytosis in a human growth hormone secretion assay (Hibino et al., 2002). Interestingly, a synergistic effect of RIMs and their binding proteins RIM-BPs was described in the calyx of Held and hippocampus synapses (Acuna et al., 2016). A combined knockout of $R I M 1 / 2$ and $R I M-B P 1 / 2$ eliminated presynaptic $\mathrm{Ca}^{2+}$-influx, tethering and priming of SVs, reduced the RRP size and functional synaptic connectivity. The RIM/RIM-BP deletion phenotype was more severe than the sum of the separate RIM1/2 or RIM-BP1/2 deletion phenotypes. This study points out that together, RIMs and RIM-BPs function as central organizers of $A Z$ function. Together, these two molecules mediate the assembly of the $A Z$ by recruiting other $A Z$ components, and participate in several steps of $A Z$ function (Acuna et al., 2016). Whether RIMs and RIM-BPs employ a similar role at IHC AZs may be tested in a combinatorial knockout approach. Further, one could investigate the expression levels of RIMs in RIM-BP2 knockout AZs and vice versa.

\section{Potential impact on RIM-BP2 - CAST/ERC (ELKS) interaction}

Besides RIMs, also CAST/ERC (ELKS) proteins might turn out to be important interaction partners of RIM-BPs. This hypothesis is based on work from Drosophila melanogaster, where disruption of the RIM-BP ortholog DRBP led to a stronger phenotype as compared to mammalian synapses. Here, presynaptic $\mathrm{Ca}^{2+}$-influx and neurotransmitter release (Liu et al., 2011; Müller et al., 2015), as well as clustering of presynaptic $\mathrm{Ca}^{2+}$-channels were 
impaired (Liu et al., 2011). In addition, the structural and functional integrity of the AZ of NMJ was severely affected and the loss of T-bars - presynaptic electron dense bodies, which tether vesicles at the NMJ (Kittel, 2006) - was observed (Liu et al., 2011). The cytomatrix protein that provides the main structural component of the T-bars, and is essential for the correct localization and density of $\mathrm{Ca}^{2+}$-channels is called Bruchpilot (Kittel, 2006). Interestingly, DRBP levels were reduced in Bruchpilot mutants, whereas overall Bruchpilot levels remained normal in DRBP mutants (Liu et al., 2011). While functional deficits in Bruchpilot and DRBP mutants share some common features, SV release is more affected in DRBP mutants (Kittel, 2006; Liu et al., 2011). In contrast, structural deficits, appear more severe in Bruchpilot mutants (Fouquet et al., 2009; Kittel, 2006). Hence, speculatively, deficits in Bruchpilot mutants could be partly explained by the accompanying loss of DRBP, which probably serves a function downstream of Bruchpilot. The N-terminus of Bruchpilot is homolog to the vertebrate CAZ protein CAST/ERC2/ELKS2 (Wagh et al., 2006). Moreover, deletion of CAST/ERC/ELKS and RIM in hippocampal neurons caused reduced protein levels of RIM-BP2, and synaptic P/Q-type $\mathrm{Ca}^{2+}$-channel immunofluorescence (Wang et al., 2016). Interestingly, both RIM1/2 and ELKS protein levels were found to be upregulated in RIM-BP1/2 deficient retinae (Luo et al., 2017), pointing towards a functional connection between RIM-BPs and CAST/ERC/ELKS either directly or indirectly through RIMs. Whether mammalian ELKS are expressed at IHC ribbon synapses and whether the interaction of CAST/ERC/ELKS and RIM-BP2 at ribbon-type synapses is structurally and functionally as relevant as in Drosophila NMJ is subject to future analysis.

\section{Impact of RIM-BP2 on sound encoding at the IHC ribbon synapse}

It is essential to identify molecular key players such as RIM-BP2 and their interaction partners to draw up a clearer picture of the complex network orchestrating synaptic transmission in IHCs. However, it is equally important to link the physiological and morphological findings in order to understand SV dynamics and their impact on sound encoding.

\section{Potential mechanism of RIM-BP2 function during vesicle replenishment}

Providing enough time for full RRP recovery (Pangršič et al., 2010), (i.e. 30-60 seconds inter-pulse-intervals), the RIM-BP2 deficient IHCs display preserved $\mathrm{Ca}^{2+}$ nanodomain-like control of exocytosis. However, in paired-pulse experiments in vitro (Figure 18) and forward masking experiments in vivo (Figure 24), RRP recovery was significantly reduced especially for short inter-stimulus-intervals (shorter than $50 \mathrm{~ms}$ ). This observation could reflect the 
requirement of RIM-BP2 for fast SV-replenishment following RRP depletion. In this process, RIM-BP2 might function as a dynamic molecular linker for newly recruited SVs, quickly guiding SVs into close proximity to a nearby $\mathrm{Ca}^{2+}$-channel. The rate-limiting step for SVs after RRP depletion could be the proper spatial positioning of newly arriving SVs in close proximity to a $\mathrm{Ca}^{2+}$-channel. The mechanism could be potentially described by a defect in "positional priming" (Neher and Sakaba, 2008). During positional priming, molecularly primed SVs move along the plasma membrane to engage with a nearby $\mathrm{Ca}^{2+}$-channel. This way, slow releasing SVs are converted into fast releasing SVs. Since the recruitment of slow releasing SVs is fast, positional priming may be rate-limiting (Neher and Sakaba, 2008). The mechanism was initially described for calyx of Held synapses and it is unclear, whether the same mechanism exists in IHCs. Positional priming was strongly associated with UNC13/Munc13, which is mainly recruited to the plasma membrane by RIM1a (Andrews-Zwilling et al., 2006; Dulubova et al., 2005; Weimer et al., 2006). However, both UNC13/Munc13 and RIM1 $\alpha$ are not expressed in IHCs (Jung et al., 2015a; Vogl et al., 2015). Moreover, positional priming was found to be actin dependent (Lee et al., 2013), which was not investigated in this work. Nonetheless, it is tempting to speculate that fast RRP-replenishment involves a RIM-BP-depending positional priming of SVs in IHCs. Moreover, the reduced availability of $\mathrm{Ca}^{2+}$-channels in RIM-BP2 deficient AZs, might further slow RRP-replenishment as SVs find fewer slots to connect. Longer RRP-recovery times might increase the likelihood of other protein-protein interactions taking place in RIM-BP2 mutants, which will eventually link the newly arriving SV to its release site.

Interestingly, in line with the hypothesis of a role of RIM-BP in positional vesicle priming, a deceleration in the resupply of high release probability SVs was observed in RIM-BP deficient Drosophila NMJ (Müller et al., 2015). The authors suggest a distinct function of DRBP in the rate-limiting conversion of SVs from the 'reserve pool' to the RRP during presynaptic homeostatic plasticity (Müller et al., 2015). In agreement, a very recent study from mouse rod bipolar cell ribbon synapses reported that RIM-BPs are important for RRP refilling as it found a RRP-replenishment deficit in RIM-BP1/2 knockout synapses (Luo et al., 2017). In mammalian central nervous system synapses, no RRP recovery deficit was found during paired-pulse stimulations with $100 \mathrm{~ms}$ (Acuna et al. 2015) or $5 \mathrm{~s}$ inter-pulse intervals (Grauel et al. 2016; Acuna et al. 2015). This is nonetheless consistent with the observed full RRP recovery for longer inter-stimulus-intervals in IHCs ( $\geq 100 \mathrm{~ms}$ ). Nonetheless, in the calyx of Held a shift from the fast component towards the slow component of release was found in RIM-BP2 deficient synapses during RRP depletion (Acuna et al. 2015), which could be due to a deficit in converting slow releasing SVs into fast releasing SVs, further arguing for a role RIM-BP2 in positional priming. 
Along these lines, it should be mentioned that the large discrepancy between $\mathrm{Ca}^{2+}$-influx and exocytosis in the sustained phase of exocytosis, where new SVs get recruited after RRP depletion, could be rescued by elevating extracellular $\left[\mathrm{Ca}^{2+}\right]_{\mathrm{e}}$ in RIM-BP2 deficient IHCs (Figure 16). Elevated extracellular $\left[\mathrm{Ca}^{2+}\right]_{\mathrm{e}}$ accelerates $\mathrm{Ca}^{2+}$-dependent SV trafficking and replenishment, which was reported for hair cells (Cho et al., 2011; Goutman and Glowatzki, 2007; Moser and Beutner, 2000; Schnee et al., 2011; Spassova et al., 2004) and other synapses (e.g. calyx of Held synapses (Hosoi et al., 2007; Wang and Kaczmarek, 1998)). Hence, lower presynaptic $\mathrm{Ca}^{2+}$-signaling due to the reduced number of synaptic $\mathrm{Ca}^{2+}$-channels in RIM-BP2 deficient IHC AZs might therefore explain their slower RRP SVreplenishment. Alternatively or in addition, an increased $\mathrm{Ca}^{2+}$-influx through elevated extracellular $\left[\mathrm{Ca}^{2+}\right]_{\mathrm{e}}$ likely reaches more distant release sites so that the exact spatial distribution and matching of $\mathrm{Ca}^{2+}-$ channels and SVs is less critical (Moser and Beutner, 2000). Elevating extracellular $\left[\mathrm{Ca}^{2+}\right]_{e}$ could thereby bypass the rate-limiting step of positional priming and exocytosis in RIM-BP2 deficient IHC synapses. Thereby, loss of RIMBP2 might decreases the temporal precision of the evoked glutamate release onto the postsynaptic SGN, leading to reduced and delayed postsynaptic firing at sound onset (Figure 24). This could be linked to the reduced wave I amplitude in the auditory brainstem recording (ABR) or the increased hearing thresholds of RIM-BP2 deficient mice (Figure 21). Since however RIM-BP2 is also knocked out in efferent olivocochlear neurons that provide inhibitory feedback onto the afferent terminals of type I SGNs (Ruel et al., 2001), effects caused through alterations in efferent modulation cannot be ruled out.

\section{RRP-replenishment from a 'reserve' SV pool}

The next question now is: Given the morphological and physiological findings of this RIMBP2 knockout study, is it possible to identify a specific pool of IHC SVs that is recruited upon RRP depletion? Conventional synapses employ synapsin I to crosslink SVs within the actin cytoskeleton in a 'reserve' SV pool to prevent them from premature fusion (reviewed in Rizzoli and Betz 2005). IHCs however lack synapsins (Safieddine and Wenthold, 1999; Vogl et al., 2015) and such a' reserve' SV pool was not specifically defined so far. But, there are indications that a sub-fraction of vesicles is clustered by actin and released during the early sustained phase of exocytosis (Guillet et al., 2016). These SVs might represent a 'reserve pool' in IHCs that is recruited upon RRP depletion (Guillet et al., 2016). Interestingly, exocytosis during 20 ms stimulation, which is thought to deplete the RRP in IHCs (Moser and Beutner, 2000), was significantly increased when pharmacologically perturbing the actin network (Guillet et al., 2016). The authors therefore proposed that SVs, which would only be available for release during longer stimulations ( $\geq 50 \mathrm{~ms}$ ) in control condition, fused with the plasma membrane ahead of time, indicating a role of actin in 
preventing premature exocytosis (Guillet et al., 2016). Thereby, actin might secure SVreplenishment at high rates and prevent SV exhaustion during the sustained phase of exocytosis by crosslinking SVs in a 'reserve pool' (Guillet et al., 2016).

More evidence that ribbon synapses employ a 'reserve pool' of SVs comes from goldfish retinal bipolar cells, where single SV fusion and dynamics could be monitored with FM1-43 dye imaging employing total internal reflection microscopy (Almers et al., 2000). The authors were able to distinguish between 'resident SVs' that are equivalent to morphologically docked SVs and fuse during the fast phase of exocytosis and newly recruited 'newcomer SVs' that fuse during the slow phase of exocytosis. Interestingly, nearly $30 \%$ of the 'newcomers' became stationary, meaning that SVs were captured in 'reserve' at a distance of $20 \mathrm{~nm}$ from the plasma membrane until a stimulus triggers their recruitment to the membrane (Almers et al., 2000). One could speculate, that this physiologically described actin-dependent 'reserve pool' (Guillet et al., 2016) and the morphologically described 'reserve pool' of SVs (Almers et al., 2000) indeed describe the same pool of SVs, which might be recruited upon RRP depletion.

How are the physiological and morphological findings in RIM-BP2 deficient IHCs related to these findings? Despite the absence of RIM-BP2, the number of MP-SVs, which are thought to form the RRP, was unaltered in IHCs (Figure 19). Interestingly, however, RIM-BP2 disruption caused a shift of a large fraction of membrane proximal SVs closer to the plasma membrane and a smaller fraction further away from the plasma membrane. The average distance of SVs from the previously defined 'reserve' SVs was $20 \mathrm{~nm}$ (Almers et al., 2000), which fits well with the observed reduced number of SVs with intermediate membrane distance ( 20-40 nm) in the absence of RIM-BP2 (Figure 20). In addition, the physiologically described defect in fast SV recruitment after RRP depletion in RIM-BP2 knockout IHCs (Figure 16 and 18) correlates well with the time-course of actin-dependent SV mobilization (Guillet et al., 2016). Hence, one could speculate that in wild type IHCs, those 'reserve pool' SVs get recruited upon RRP depletion and that this recruitment process might require RIMBP2. One could however also speculate that SVs get recruited to the release sites in a lateral fashion from more distant locations towards the ribbon center (Lenzi et al., 2002). The lateral distribution of MP-SVs with respect to the PD was unaltered in $R I M-B P^{-/} \mathrm{AZs}$, which might explain the mild effect of RIM-BP2 disruption on the RRP release. However, "outlying"-SVs were shifted further away from the PD in RIM-BP2-/ AZs. Therefore, one could propose that those "outlying"-SVs get laterally recruited to the release sites adjacent to the synaptic ribbon during the sustained phase of release. Upon loss of RIM-BP2 this lateral SV recruitment to the release sites could be impaired, leading to an increased distance of SVs to the PD. Therefore, such lateral recruitment might be an additional mechanism to consider. 


\section{Potential molecular mechanism of RIM-BP2 at IHC AZs}

Generally, one should keep in mind, that $\mathrm{Ca}^{2+}$-imaging and immunohistochemical staining in RIM-BP2 deficient IHCs pointed towards a 40-50\% decrease of synaptic $\mathrm{Ca}^{2+}$-channels (Figure 14 and 15). Whether both, the reduced number of $\mathrm{Ca}^{2+}$-channels and slowed RRPreplenishment (Figure 18 and 24) from SVs of an 'reserve pool' contribute to the significantly reduced sustained rate of exocytosis (Figure 16) individually or whether the observed replenishment defect is a consequence of reduced $\mathrm{Ca}^{2+}$-channels needs further elucidation. One could imagine that upon loss of synaptic $\mathrm{Ca}^{2+}$-channels, availability of SV release slots is reduced at mutated AZs (illustrated in Figure 25). Nonetheless, release of RRP SVs was affected only mildly (Figure 16), as presumably a sufficient number of release sites was still present (Figure 25A). Moreover, in the absence of RIM-BP2, RRP SVs remained tightly coupled to the remaining $\mathrm{Ca}^{2+}$-channels in a nanodomain-like manner when analyzed in hyperpolarized IHCs, where sufficient recovery time was provided (Figure 17). In a more dynamic scenario during prolonged IHC stimulation, where SV s have to engage with a $\mathrm{Ca}^{2+}$ channel quickly, the availability of $\mathrm{Ca}^{2+}$-channels and release sites might however be rate limiting (Figure 25B). The additional absence of a molecular linker like e.g. RIM-BP2 that guides SVs to the remaining $\mathrm{Ca}^{2+}$-channels might further enhance the deceleration of SVreplenishment (Figure 25B). Given the possibility of RIM-BP2 interaction with the ribbon anchor bassoon (Davydova et al., 2014) another conceivable interpretation for the slow RRP-replenishment upon RIM-BP2 disruption could be a slightly increased distance between $\mathrm{Ca}^{2+}$-channels and the presynaptic density and thus, the ribbon-tethered SVs (Figure 25B). Both, STED microscopy and $\mathrm{Ca}^{2+}$-imaging did not reveal changes in the width and area of Cav1.3 clusters and spread of the $\mathrm{Ca}^{2+}$ signal (Figure 14 and 15), arguing against major changes in $\mathrm{Ca}^{2+}$-channel localization. However, a slight increase of the distance of $\mathrm{Ca}^{2+}$-channels from the PD cannot be ruled out and could slow down the engagement of newcoming ribbon-tethered SVs with a $\mathrm{Ca}^{2+}$-channel during RRPreplenishment (Figure 25B). Thereby, SV turnover speed would not only be limited by a lower probability of $\mathrm{SV}$ - release site matching due to a reduced number of $\mathrm{Ca}^{2+}$-channels, but also through the slowed engagement of SVs with the remaining $\mathrm{Ca}^{2+}$-channels (Figure 25B). This argues for a dual role of RIM-BP2 in IHC synaptic transmission, whereby RIMBP2 might potentially function as a molecular hub between L-type $\mathrm{Ca}^{2+}$-channels and/or Bassoon, RIM2/Rab3, and synaptic vesicles. 
A

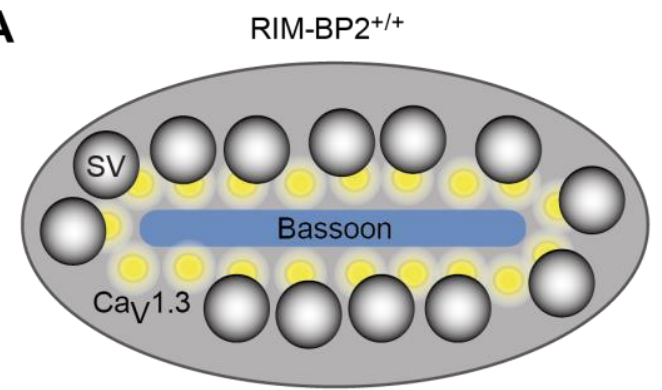

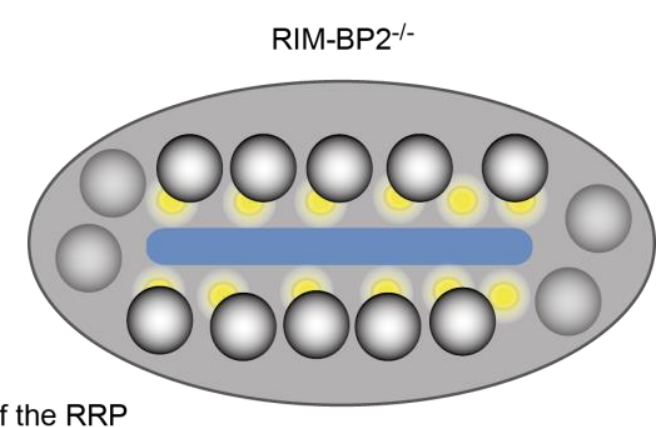

Exocytosis of the RRP

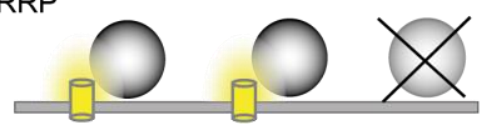

B

Vesicle recruitment after RRP depletion

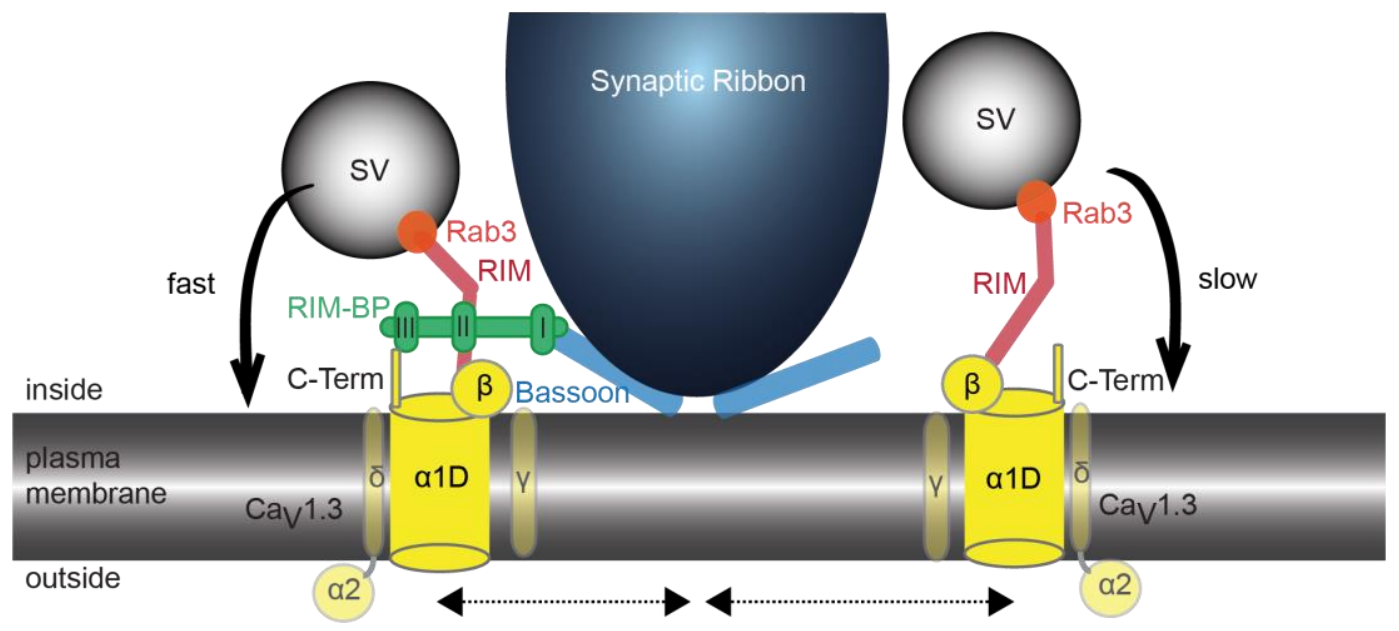

Figure 25: Schematic summary of RIM-BP2 function at IHC AZs

[A] A lower number of Cav1.3 $\mathrm{Ca}^{2+}$-channels (yellow) was found to be present in the absence of RIM-BP2, whereas the number of MP-SVs (SV) and their distance to the PD (blue) remained unaltered. The reduction of $\mathrm{Cav} 1.3 \mathrm{Ca}^{2+}$-channels likely explains the mildly reduced RRP exocytosis. [B] During the sustained phase of SV release, RRP SVs are replenished and engage with a nearby $\mathrm{Ca}^{2+}$-channel (left side of the scheme). In the absence of RIM-BP2, RRP-replenishment was slowed (right side of the scheme). Mechanistically this could be explained (1) by a lower probability of SV - release site matching due to a reduced number of $\mathrm{Ca}^{2+}$-channels, (2) by a slightly increased distance of newcoming SVs to a $\mathrm{Ca}^{2+}$-channel (dashed arrows) possibly because of the lacking RIMBP2 (green) - bassoon (blue) interaction and/or (3) by a role of RIM-BP2 in "positional priming" by mediating fast engagement of a newcoming SV with a $\mathrm{Ca}^{2+}$-channel, possibly through RIM-BP2 - RIM (red) - Rab3 (orange) interaction. 


\section{Chapter 2}

"Establishing voltage imaging in spiral ganglion neurons" 


\section{$5.1 \quad$ Introduction}

While each presynaptic $A Z$ of a given IHC is driven by the same receptor potential, the postsynaptic responses of SGNs differ i.e. in their spontaneous firing rate, threshold, and dynamic range (Liberman, 1978; Ohlemiller and Echteler, 1990; Sachs and Abbas, 1974; Taberner and Liberman, 2005; Yates et al., 1990). Likely this diversity is a mechanism to encode sounds of varying intensity. There is accumulating evidence that presynaptic heterogeneity in IHC AZs at least partially underlies the diversity of SGN responses as described in the General Introduction. However, beyond $\mathrm{Ca}^{2+}$-signaling (Frank et al., 2009; Meyer et al., 2009; Ohn et al., 2016) so far functional differences among the synapses have not yet been studied and a causal link of presynaptic heterogeneity to SGN response diversity remains to be demonstrated. Addressing presynaptic heterogeneity on the level of SV exocytosis at individual AZs by pH-sensitive pHluorin (Miesenböck et al., 1998; Sankaranarayanan et al., 2000) turned out to be challenging in IHCs, because the intraluminal $\mathrm{pH}$ of vGlut1-pHluorin positive organelles was less acidic ( 6.5) compared to conventional presynaptic terminals ( 5.5) (Miesenböck et al., 1998; Neef et al., 2014). pH sensitive fluorescence proteins are generally suitable for monitoring SV fusion, due to the $\mathrm{pH}$ gradient between the vesicular compartments and the extracellular space. While the fluorescence signal of pHluorin is quenched inside the acidic SV lumen $(\mathrm{pH} 5-6)$, upon externalization of pHluorin molecules during SV fusion, proton quenching is released due to the neutral extracellular space and its fluorescence signal increases. However, the relatively neutral vesicular $\mathrm{pH}$ in $\mathrm{IHCs}$, lead to rather small changes of pHluorin fluorescence signals upon SV exocytosis (Neef et al., 2014). Postsynaptic patch-clamp recordings from SGN terminals elicited important details about hair cell transmitter release from a single $A Z$, about spike timing, amplitude, and spontaneous rates (Glowatzki and Fuchs, 2002; Rutherford et al., 2012). In paired in vitro patch-clamp recordings of IHCs and SGN boutons a high variability in EPSC shape and amplitude was observed (Glowatzki and Fuchs, 2002; Rutherford et al., 2012). But it is experimentally difficult to access several postsynaptic boutons of SGNs contacting a single IHC by multiple in vitro patch-clamp recordings to simultaneously characterize the response of several SGNs to the same stimulus (Wu et al., 2016). To date no more than two boutons innervating the same IHC have been recorded (Wu et al., 2016), which highlights the difficulty and limitations of this technique when aiming to probe the heterogeneous responses characteristic of postsynaptic SGNs. Nevertheless, this experimental approach enabled the authors to demonstrate that, indeed, SGNs with different spike properties innervate the same IHCs in the rat cochlea (Wu et al., 2016). 
In order to explore heterogeneous responses characteristic of several postsynaptic SGNs contacting the same IHC on a single synapses level, the application of a genetically encoded voltage indicator (GEVI) in SGNs was chosen. Optical detection of APs with voltage indicators is a non-invasive approach to study neuronal and network activity, but it remained challenging for a long time. Multiple challenges, e.g. intracellular injection of voltage-dyes, membrane localization of genetically encoded voltage indicators, brightness, kinetics, voltage-sensitivity, signal-to-noise-ratio, and undesirable hyperpolarizing photocurrents of voltage indicators had to be faced and optimized (reviewed in Kulkarni and Miller 2017; Y. Xu, Zou, and Cohen 2017). The discovery of the voltage sensor ArcLight Q239 (Jin et al., 2012) was a breakthrough in the field. It is highly sensitive (35\% $\Delta \mathrm{F} / \mathrm{F}$ per $100 \mathrm{mV}$ ) to membrane voltage changes, but could only resolve neuronal APs up to $60 \mathrm{~Hz}$ ( $\tau$ between -70 and $+30 \mathrm{mV}$ : AcrLight Q239 $\tau_{\text {on }} 9 \mathrm{~ms}$, $\tau_{\text {off }} 15 \mathrm{~ms}$ ) (Piao et al., 2015). The genetically encoded archaerhodopsin-based voltage indicators QuasAr1, 2 (Hochbaum et al., 2014), and 3 (unpublished, Prof. Adam Cohen) were engineered from the microbial rhodopsin protein, Archaerhodopsin 3 (Arch) from Halorubrum sodomense (Kralj et al., 2011). Besides improved brightness and voltage sensitivity ( $\Delta \mathrm{F} / \mathrm{F}$ per $100 \mathrm{mV}$ : QuasAr2 90\%, QuasAr1 33\%), they lack endogenous proton-pumping photocurrent and are characterized by fast response kinetics ( $\tau$ between -70 and $+30 \mathrm{mV}$ : QuasAr1 $\tau_{\text {on }} 0.05 \mathrm{~ms}$, $\tau_{\text {off }} 0.07 \mathrm{~ms}$; QuasAr2 $\tau_{\text {on }} 1.2 \mathrm{~ms}$, $\tau_{\text {off }} 1.0 \mathrm{~ms}$ ) (Hochbaum et al., 2014; Kralj et al., 2011). Nonetheless, Arch-derived GEVIs are typically 30-80-fold dimmer than GFP and require high near-infrared illumination intensities (200-1000 W/cm²) (Hochbaum et al., 2014; Kralj et al., 2011). Recently developed versions of the blue-shifted GEVI Ace-mNeon (Ace1Q$\mathrm{mNeon}$ and Ace2N-(4AA)-mNeon) showed remarkable brightness and speed ( $\tau$ between 70 and $+30 \mathrm{mV}$ : Ace-2N-mNeon $\tau_{\text {on }} 0.36 \mathrm{~ms}, \tau_{\text {off }} 0.42 \mathrm{~ms}$ ), which allowed single-cell and single-AP resolution in mouse and fly brains in vivo (Gong et al., 2015).

However, QuasArs are more suitable for a combination with blue-light optogenetic stimulation or $\mathrm{Ca}^{2+}$-imaging due to their near-infrared fluorescence spectrum. Particularly, combination of QuasAr imaging with presynaptic $\mathrm{Ca}^{2+}$-imaging would allow a direct correlation of presynaptic IHC AZ properties and postsynaptic SGN response characteristics on a single synapse level. Moreover, optogenetic stimulation would allow exploring the neuronal SGN behavior independent of sensory IHC stimulation. Hence, optical stimulation would be a useful tool to investigate whether the presynaptic input, or the intrinsic properties of the SGNs themselves shape their heterogeneous response characteristics, given homogeneous expression of CheRiff as found in transgenic mice (Lou et al., 2016). "All-optical electrophysiology" was performed with the GEVI QuasAr2 as effector and the blue-shifted channelrhodopsin CheRiff as actuator both in acute brain slices 
and in vivo (Hochbaum et al., 2014; Lou et al., 2016). It allowed qualitative classification of neurons based on their firing pattern and the detection of individual APs upon activation of CheRiff (Hochbaum et al., 2014; Lou et al., 2016). Besides the availability of a transgenic mouse line co-expressing QuasAr2 and CheRiff (Lou et al., 2016), virus mediated gene transfer can be used for transgene expression in SGNs. Virus mediated gene transfer using AAV2/6 successfully expressed the channelrhodopsin CatCh in SGNs and rendered SGNs light-sensitive (Hernandez et al., 2014). Hence, AAV2/6 mediated gene expression is highly suitable to express GEVIs in SGNs and was therefore employed in this work.

\section{$5.2 \quad$ Results}

In this chapter, the feasibility of the use of GEVIs QuasAr2 and QuasAr3 as optical readout systems for spike detection in SGNs was determined. For that, a confocal microscope, optimized for presynaptic $\mathrm{Ca}^{2+}$-imaging, was enhanced with a custom wide field imaging set up to carry out voltage imaging in the far-red fluorescence imaging spectrum. Further, QuasAr2 and QuasAr3 expression were examined in cochleae of Floxpatch-Bhlhb5-Cre mice and in postnatally AAV2/6-transduced mice, respectively. Finally, the capability of detecting membrane-voltage changes by fluorescence was tested in both expression systems by employing two different stimulation approaches. First, optical SGN stimulation by blue light induced depolarizing photocurrents through CheRiff and membrane voltage readout through QuasAr2 and QuasAr3 was tested. Second, presynaptic IHC patch-clamp stimulation was performed to stimulate SGNs indirectly through exocytic IHC glutamate release, while QuasAr3 fluorescence in postsynaptic SGN boutons was recorded simultaneously.

\section{Expression of QuasAr2 and all-optical electrophysiology in the cochlea of Floxpatch- Bhlhb5-Cre mice}

An advantage of genetically encoded voltage indicators over synthetic dyes is their ability to be specifically targeted to a subset of cells in tissue. Compared to virus-mediated expression, transgenic mouse lines show more uniform expression levels, which might be especially important for optical reporters or stable photocurrents during quantitative optogenetic stimulation (Gong et al., 2007; Harris and Dallos, 1979; Madisen et al., 2015). The Cre-dependent transgenic Optopatch2 mouse line, named Floxpatch (Lou et al., 2016), was crossed with a Bhlhb5-Cre driver line for Cre-mediated excision of the premature Stop codon and thereby tissue-specific expression of the Optopatch2 construct in neurons and hence, SGNs of the cochlea (Ross et al., 2010). Floxpatch mice express the GEVI QuasAr2 
as well as CheRiff, a blue-shifted channelrhodopsin tagged with eGFP (Hochbaum et al., 2014; Lou et al., 2016). Endogenous eGFP fluorescence was visible in Floxpatch-Bhlhb5$\mathrm{Cre}^{+}$mouse cochleae (Figure 26a, side view), especially in the Rosenthal's canal, where SGN somata are located, and was absent in Floxpatch-Bhlhb5-Cre mice (Figure 26b). Specific anti-GFP antibody staining in Floxpatch-Bhlhb5-Cre ${ }^{+}$(Figure 26a'), but not Floxpatch-Bhlhb5-Cre- (Figure 26b') mouse organs of Corti further validated the expression of eGFP in SGNs. Since the sequences coding for QuasAr2 and CheRiff-eGFP are linked by a P2A sequence for stoichiometric co-expression (Lou et al., 2016), one can conclude that QuasAr2 should be expressed in Floxpatch-Bhlhb5-Cre+ ${ }^{+}$cochlea SGNs.

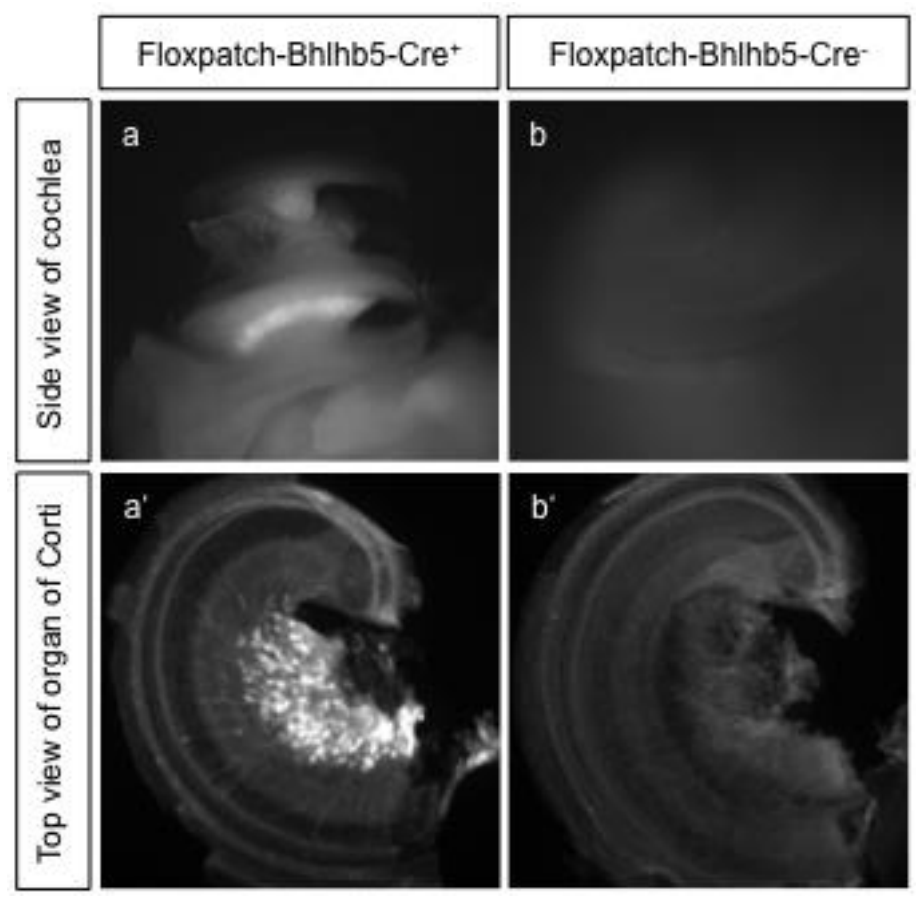

Figure 26: Optopatch2 expression in the cochlea of Floxpatch-Bhlhb5-Cre mice

A Cre-dependent transgenic Optopatch2 mouse line, named Floxpatch (Lou et al., 2016) was crossed with a Bhlhb5-Cre driver line for specific expression of QuasAr2 and CheRiffeGFP in SGNs (Ross et al., 2010). Endogenous eGFP fluorescence was visible in a side view of the dissected cochlea of Floxpatch-Bhlhb5-Cre+ mice (a) and was absent in Floxpatch-Bhlhb5-Cre- mice (b). Specific anti-GFP antibody staining in Floxpatch-Bhlhb5$\mathrm{Cre}^{+}\left(\mathrm{a}^{\prime}\right)$, but not Floxpatch-Bhlhb5-Cre (b') mouse organs of Corti further validated the expression of Optopatch2 in SGNs. This experiment was performed in the laboratory of Prof. Adam Cohen, Harvard University, and Prof. Lisa Goodrich, Harvard Medical School.

The Optopatch2 construct expressed in Floxpatch-Bhlhb5-Cre mice contains the channelrhodopsin CheRiff and therefore allows optical stimulation of SGNs by blue lightinduced photocurrents. Hence, SGNs were stimulated optically with laser pulses of increasing intensity and QuasAr2 fluorescence was acquired simultaneously to monitor changes in membrane voltage and optically induced APs. QuasAr2 imaging experiments were carried out in the laboratory of Prof. Adam Cohen, Harvard University. Apical turns of 
the organ of Corti from transgenic Floxpatch-Bhlhb5-Cre ${ }^{+}$mice (Figure 8 and Figure 27) were freshly dissected and SGN boutons were stimulated by 500 ms pulses of increasing $488 \mathrm{~nm}$ laser irradiance $\left(0.6\right.$ to $\left.3 \mathrm{~W} / \mathrm{cm}^{2}\right)$ at a custom made wide-field imaging microscope (Hochbaum et al., 2014; Kralj et al., 2011; Lou et al., 2016). QuasAr2 fluorescence was recorded with a camera frame rate of $1 \mathrm{kHz}$ and regions of interest at several SGN boutons were manually chosen for analysis (Figure 27B). Apart from optical crosstalk induced by $488 \mathrm{~nm}$ laser wide-field illumination (Hochbaum et al., 2014), no fluorescence changes in membrane voltage or APs were detected (Figure 27B).

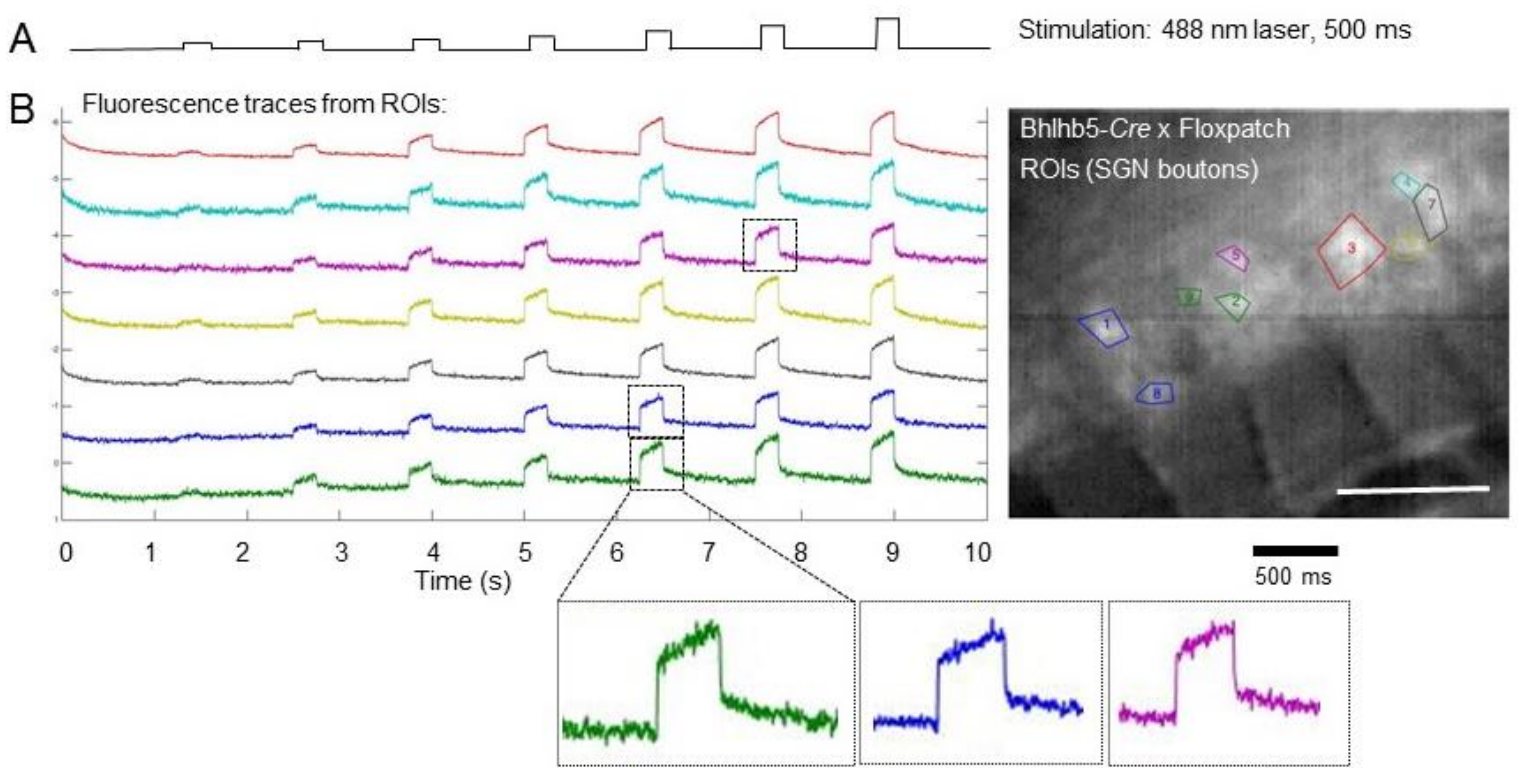

Figure 27: Optopatch2 experiment in SGN boutons of a Floxpatch-Bhlhb5-Cre ${ }^{+}$ mouse organ of Corti

SGN boutons from a freshly dissected organ of Corti of a Floxpatch-Bhlhb5-Cre ${ }^{+}$mouse ([B] right) were optically stimulated with $500 \mathrm{~ms}$ pulses of increasing $488 \mathrm{~nm}$ laser irradiance (from 0.6 to $3 \mathrm{~W} / \mathrm{cm}^{2}$, illustrated in $[\mathrm{A}]$ ). QuasAr2 fluorescence was acquired with a frame rate of $1 \mathrm{kHz}$ and different regions of interest (ROls) ([B] right, scale bar: $10 \mu \mathrm{m}$ ) were chosen for analysis. For each ROI, the mean fluorescence was plotted over time ([B] left). The increase in QuasAr2 fluorescence during blue laser illumination most likely represents optical crosstalk (Hochbaum et al., 2014). No additional fast QuasAr2 fluorescence changes, i.e. voltage-spikes, were detected. This experiment was performed in the laboratory of Prof. Adam Cohen, Harvard University.

One reason for the absence of fluorescently detected membrane voltage changes by the use of Optopatch2 could be a relatively low expression level of the optical actuator CheRiff and reporter QuasAr2 in the transgenic mouse line compared to virus-mediated overexpression. It appears likely that higher expression levels of QuasAr2 are more capable of robustly detecting neuronal activities. Further, higher expression levels of CheRiff might 
increase light-induced photocurrents through a higher number of channels in the membrane.

\section{Expression of QuasAr3 in the postnatally virus-transduced mouse cochlea}

Therefore, virus-mediated gene expression of the channelrhodopsin CheRiff and the voltage indicator QuasAr3(Q95H), combined in the Optopatch3 gene construct (Figure 9, unpublished, by the laboratory of Prof. Adam Cohen, Harvard University) was employed next. In addition to higher expected expression levels through virus-mediated transgene overexpression, the Optopatch3 construct is supposed to offer superior expression levels compared to Optopatch2, because trafficking signal motifs (TSX3 and TS) and an endoplasmic reticulum export sequence (ER2) were inserted for both transgenes to enhance their protein trafficking and expression at the membrane surface.

To express the Optopatch3 construct in SGNs, the Optopatch3 DNA construct was packed into AAV2/6 (produced by North Carolina Core facility), as virus-mediated gene transfer using AAV2/6 was previously shown to successfully target SGNs in the cochlea (Hernandez et al., 2014). The Optopatch3 AAV2/6 virus was postnatally injected into mouse cochleae via the round window (Akil et al., 2012) (injection performed by Christiane Senger-Freitag). The virus was injected at postnatal day p5 and QuasAr3 expression was examined $\sim 10$ days later, shortly after hearing onset at p14-p16.

QuasAr3 was fused to citrine a variant of the GFP mutant YFP (yellow fluorescent protein), and hence detected by anti-GFP antibodies (Nagai et al., 2002). Therefore, QuasAr3 expression could be determined via immunohistochemical labeling with an anti-GFP antibody. Since in each animal the virus was injected into only one cochlea, the other noninjected cochlea served as control. Hence, injected and non-injected control cochleae were cut into $16 \mu \mathrm{m}$ thick cryo-sections. Mid-modiolar cross sections were labeled with anticalretinin and anti-GFP antibodies and imaged with confocal microscopy (Figure 28). Thereby, an overall impression of the expression pattern of QuasAr3 after postnatal AAV2/6 injection in the round window of the cochlea was achieved and the transduction efficiency of the AAV2/6 injection was estimated. The transduction efficiency of cochleae after postnatal virus injection was determined by the presence of GFP-positive SGN somata and amounted 56\% (16 injected mice from 2 litters). Co-immunohistochemical labeling with calretinin was performed, since calretinin is an endogenous $\mathrm{Ca}^{2+}$-buffer of IHCs (Pangršič et al., 2015) and type I, but not type II SGNs (McLean et al., 2009). 

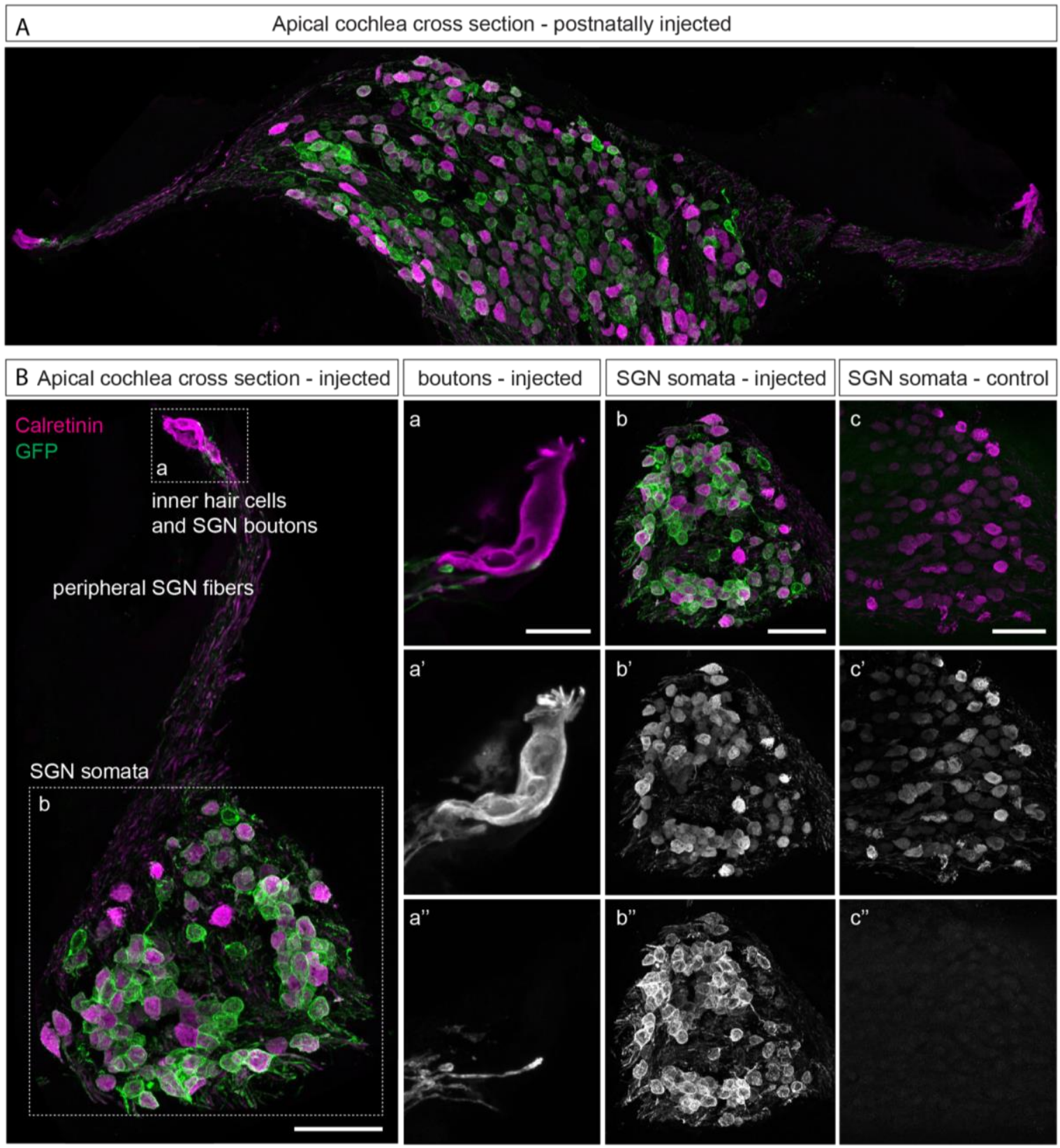

Figure 28: Expression of QuasAr3 in the cochlea after postnatal AAV2/6 injection

Confocal images of cryo-sections of an AAV2/6-transduced mouse cochlea. Cochleae were injected postnatally at p5 and fixed at p15. QuasAr3 expression was determined by immunolabeling with an anti-GFP antibody (green) detecting the QuasAr3-citrine fusion construct. An anti-calretinin antibody (magenta) visualized type I SGNs and IHCs (McLean et al., 2009; Pangršič et al., 2015). [A] Overview cross section through an apical cochlea turn. Image center shows SGN somata in the Rosenthal's canal. Peripheral fibers radiate towards the IHCs on both sides of the image. [B] Cross section through an apical cochlear turn (left, scale bar: $50 \mu \mathrm{m}$ ) and individual close-up images (right) of SGN boutons adjacent to an IHC (a-a"', scale bar: $10 \mu \mathrm{m})$ and SGN somata (b-b"', scale bar: $50 \mu \mathrm{m})$ from an AAVinjected animal as well as SGN somata of a non-injected control cochlea (c-c", scale bar: $50 \mu \mathrm{m})$. a, b, and c show merged confocal images of calretinin immunofluorescence (") and GFP immunofluorescence ("). 
Figure 28A shows an overview cross section through the apical cochlea turn of an exemplary AAV2/6 - injected cochlea. A large number of type I SGNs were GFP-positive, indicating high AAV2/6 transduction rates (Figure 28A). The calretinin/GFP merge image shows SGN somata in the center and their peripheral axons projecting towards the also calretinin-positive IHCs. Since live-cell voltage imaging experiments will be mainly performed in the apical cochlear region, Figure 28B shows a merged overview image of the apical cochlear turn (left) and individual close-up images (right) of SGN boutons adjacent to an IHC (a-a"), SGN somata (b-b"), and in addition SGN somata of a non-injected control cochlea (c-c"). GFP signal was clearly present both in boutons at the IHC base (a") and SGN somata (b") and was absent in non-injected SGN somata (c"). QuasAr3-citrine fluorescence was highly membrane-localized, which was best visible in SGN somata when comparing the distribution of GFP fluorescence signal (b, b") to the cytosolic calretinin fluorescence signal (b, b').

Next, the expression of QuasAr3 after postnatal injection was examined in SGN fibers and boutons adjacent to the IHCs in whole-mounts of freshly dissected organs of Corti. Antibody staining was performed against the IHC-specific protein vGlut3 (blue) (Seal et al., 2008) and the type I and II SGN-specific protein NF200 (magenta) (McLean et al., 2009) (Figure 29). The anti-GFP antibody fluorescence (green) revealed QasAr3-citrine expression in SGN boutons adjacent to IHCs (Figure 29, asterisk).

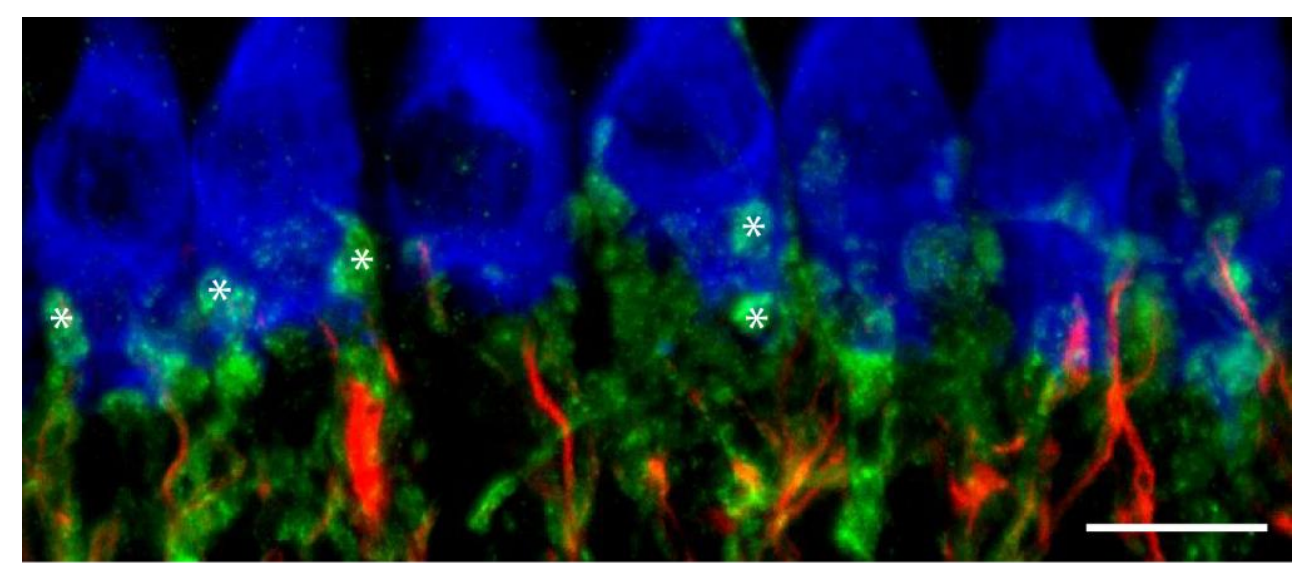

Figure 29: QausAr3-Citrine expression in SGN boutons

Maximum projection of confocal sections from a whole-mount of a freshly dissected organ of Corti of an AAV2/6 transduced mouse cochlea. Virus was injected postnatally at p5 and the organ of Corti was fixed at p15. QuasAr3 expression was determined by immunolabeling with an anti-GFP antibody (green) detecting the QuasAr3-citrine fusion construct. IHCs were labeled with anti-vGlut3 antibody (blue) (Seal et al., 2008), type I and II SGN were labeled with an anti-NF200 antibody (red) (McLean et al., 2009). Asterisks highlight some SGN boutons, which show strong expression of QuasAr3-citrine and which are adjacent to IHCs. Scale bar: $10 \mu \mathrm{m}$. 
In order to distinguish QuasAr3-expressing from non-QuasAr3-expressing organs of Corti after postnatal virus injection before live-cell imaging experiments in non-fixed tissue, baseline QuasAr3 fluorescence was measured in freshly dissected organs of Corti during excitation with a $637 \mathrm{~nm}$ laser at different intensities (1 - $140 \mathrm{~mW}$ ) (Figure 30). QuasAr3 fluorescence was clearly visible (Figure 30A) by $637 \mathrm{~nm}$ laser illumination in the custommade wide-field imaging microscope (Figure 10). SGN boutons of non-injected control organs of Corti showed auto-fluorescence signals that looked qualitatively similar to QuasAr3 fluorescence in boutons. The intensity of QuasAr3 baseline fluorescence however, differed remarkably (increased by a factor of 1000) from background fluorescence (Figure 30A, B). Validation of QuasAr3 expression by determining QuasAr3 baseline fluorescence at different laser intensities was confirmed by anti-GFP antibody staining (as in Figure 29) and was consistent. Therefore, positive virus-transduction of SGNs was determined by measuring the QuasAr3 fluorescence intensity range before each voltage imaging experiment.
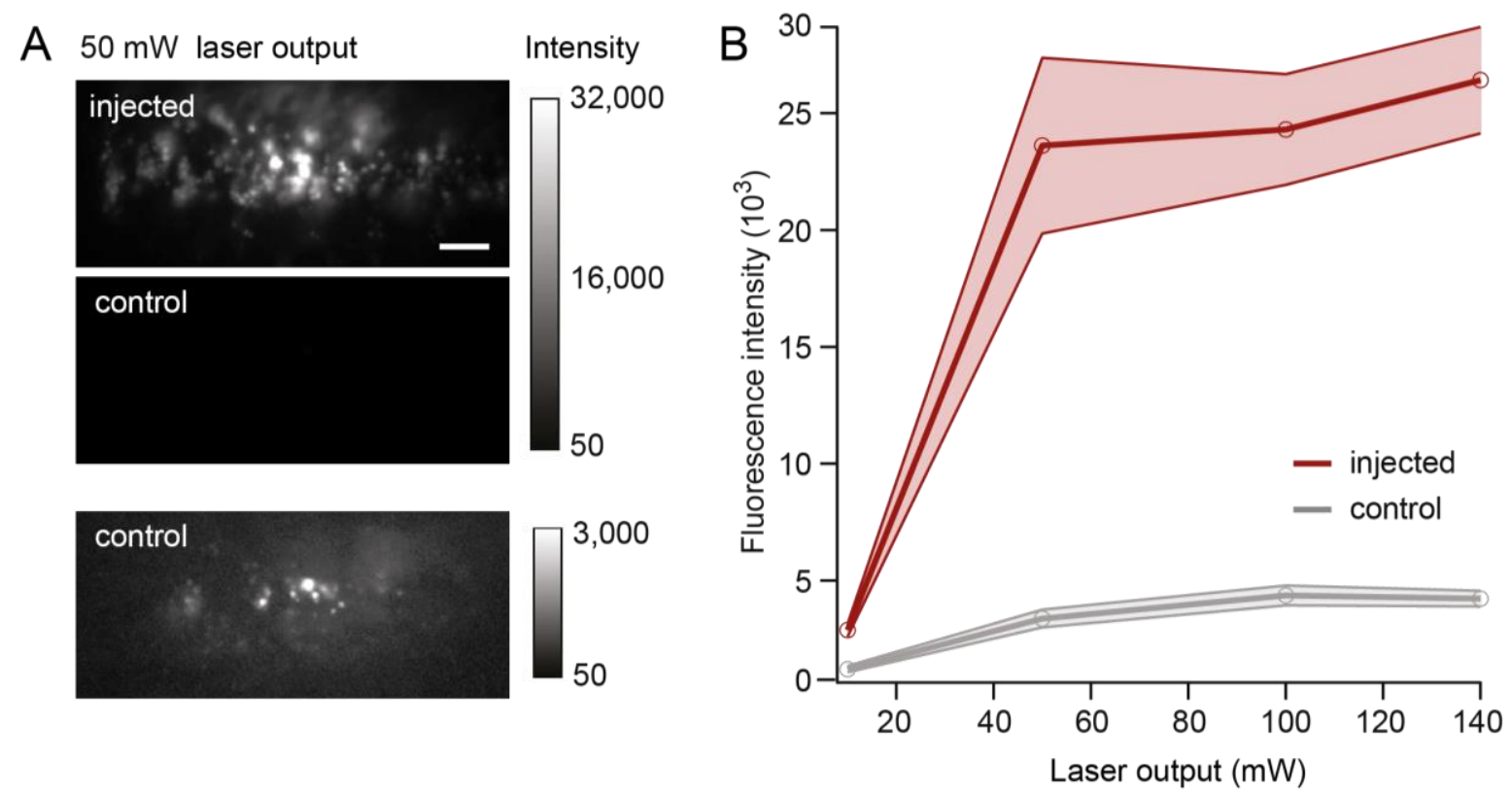

Figure 30: Baseline fluorescence of QuasAr3 expressing boutons

QuasAr3 fluorescence was clearly visible by $637 \mathrm{~nm}$ laser illumination in the custom-made wide-field imaging microscope. [A] Top view on several boutons (white spots) in a freshly dissected apical turn of a virus injected (top) and non-injected control (middle and bottom) organ of Corti (scale bar $5 \mu \mathrm{m}$ ) acquired at $50 \mathrm{~mW} 637 \mathrm{~nm}$ laser output. Top and middle image are displayed with the same intensity lookup table. Fluorescence intensities of boutons from the injected organ of Corti differ remarkably from the auto-fluorescence in non-injected control boutons. Control boutons are additionally displayed with an adjusted lookup table for comparison of fluorescence patterns (bottom). Positive virus transduction of the injected cochleae was confirmed by immunohistochemistry (as in Figure 29). [B] Fluorescence intensities were quantified by measuring the average fluorescence intensity of $n=15-30$ boutons from $N=2$ injected and control mice, each. Data represent mean $\pm S E M$. Age of mice: $p 15$. 


\section{Wide-field illumination setup}

Together with Dr. Kai Bodensiek, I established a custom-built wide-field imaging setup (see Materials and Methods, Figure 10), by modifying a preexisting patch-clamp setup equipped with a laser-scanning microscope. Dichroic mirrors and a separate excitation laser coupling port enabled wide-field imaging without interfering with the preexisting laser scanning microscope beam path. The HEKA patch-clamp amplifier was used as master to synchronize illumination, stimulation, camera acquisition, and the collection of confocal images.

To gain high laser intensities and homogeneous wide-field illumination of the sample, the beam of a $637 \mathrm{~nm}$ diode laser was expanded with a lens system and size-modulated by an iris. When illuminating a densely packed sample of TetraSpeck Microspheres, a sample illumination spot (white dashed line) with approximately $75 \mu \mathrm{m}$ diameter was measured (Figure 31A). Converting the measured laser output from a photometer onto the illuminated area, an average laser irradiance of up to $4000 \mathrm{~W} / \mathrm{cm}^{2}$ was reached at maximal laser output of $140 \mathrm{~mW}$. In a recent study, laser irradiance for QuasAr2 imaging ranged from $100 \mathrm{~W} / \mathrm{cm}^{2}$ in neuronal cultures, $1200 \mathrm{~W} / \mathrm{cm}^{2}$ in organotypic slices (Hochbaum et al., 2014) up to 2500 $\mathrm{W} / \mathrm{cm}^{2}$ in dorsal root ganglia in vivo (Lou et al., 2016). Therefore, during QuasAr3 voltage imaging experiments in SGNs mostly $30 \mathrm{~mW}$ laser output intensity was used, as this was sufficient to reach a high average sample irradiance of $1200 \mathrm{~W} / \mathrm{cm}^{2}$. Homogeneity of the laser beam profile was examined by illuminating a homogenous Alexa-633 solution (Figure $31 \mathrm{~B})$. No speckles were visible, and the beam profile showed a uniform Gaussian distribution (red lines) (Figure 31B).

For rapid detection of small fluorescence changes of QuasAr3, a sCMOS camera (OrcaFlash4.0V2) was employed. With a calculated 60.7x magnification factor (60x water immersion objective) determined by a micrometer scale, one pixel on the camera chip with a size of $6.5 \mu \mathrm{m}$ converted into $107 \mathrm{~nm}$ on the sample. To reach high image acquisition rates of $1 \mathrm{kHz}$, the camera readout area was restricted to $2048 \times 184$ pixels (white solid box) symmetric to the camera chip centerline (white solid line) (Figure 31C) using binning of $2 \times 2$ pixels. Both, the laser illumination spot and the readout area of the camera restrict the available imaging field. Nonetheless, the effective readout area is approximately $75 \mu \mathrm{m}$ $x 20 \mu \mathrm{m}$ (Figure 31D), sufficient to image the fluorescence of several boutons (red circles) of one IHC (dashed white lines) or from several IHCs simultaneously (Figure 31D). 

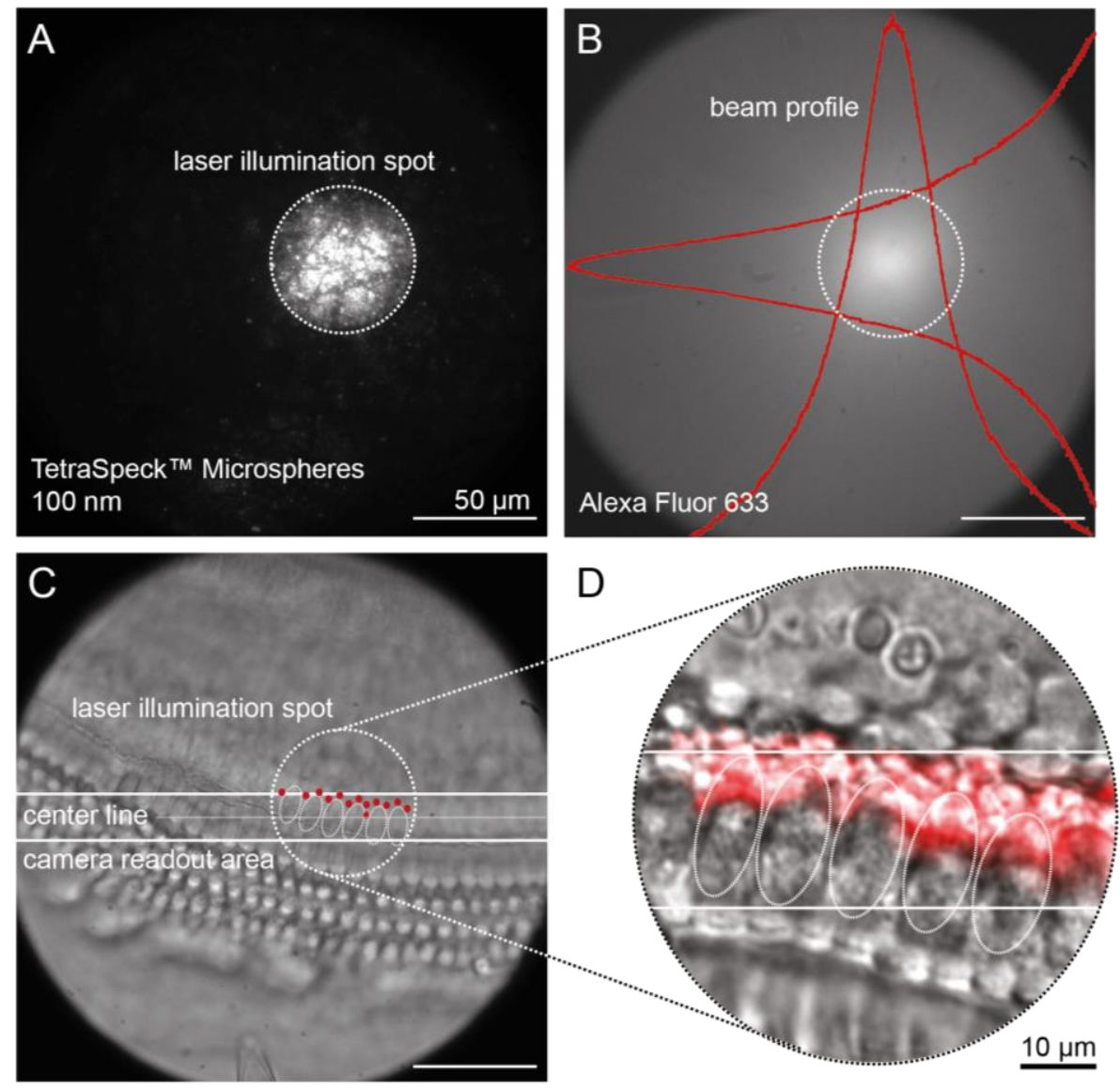

Figure 31: Wide-field illumination setup

The custom-built wide-field imaging setup was established together with Dr. Kai Bodensiek. [A] Expanded and size-modulated $637 \mathrm{~nm}$ diode laser beam illuminating a circular region of approximately $75 \mu \mathrm{m}$ diameter (dashed lines) within a sample of densely packed TetraSpeck Microspheres. Scale bar $50 \mu \mathrm{m}$. [B] The laser beam profile was investigated by illuminating a homogenous Alexa-633 solution. The beam profile showed a uniform Gaussian distribution (red lines). Scale bar $50 \mu \mathrm{m}$. [C] Bright-field image of a freshly dissected mouse organ of Corti. The image was acquired while focusing on the IHC hair bundles. Dashed lines indicate the locations of IHCs. Red dots indicate the locations of SGN terminals innervating the IHCs. To reach high image acquisition rates of $1 \mathrm{kHz}$, the camera readout area was restricted to $2048 \times 184$ pixel (white solid box) symmetric to the camera chip centerline (white solid line). Scale bar: $50 \mu \mathrm{m}$. [D] Enlarged view on the laser illuminated area from the freshly dissected mouse organ of Corti in [C], focused towards the IHC base. Dashed lines indicate IHCs and white box indicates the camera readout area during voltage imaging. A fluorescent wide-field image (red) of a virus transduced QuasAr3 expressing mouse was merged with the bright-field image (grays) and roughly indicates the location of SGNs. Scale bar: $10 \mu \mathrm{m}$.

Further, the $637 \mathrm{~nm}$ laser power stability was tested for different laser modulations and intensities to minimize possible optical fluctuation effects induced by the laser (Figure 32). Together with Dr. Kai Bodensiek, a custom-built photodiode circuit was used to record laser power with a sampling rate of $20 \mathrm{kHz}$. The measured photodiode output did not linearly correlate with the laser power, but provided a high temporal resolution to detect laser fluctuations. Laser power was measured in the analog and digitally triggered constant power 
(APC) mode (Figure 32A and B), and digitally triggered constant current (CC) mode (Figure $32 \mathrm{C})$ for three different laser intensities (10 mW, $75 \mathrm{~mW}$ and $100 \mathrm{~mW}$ ).

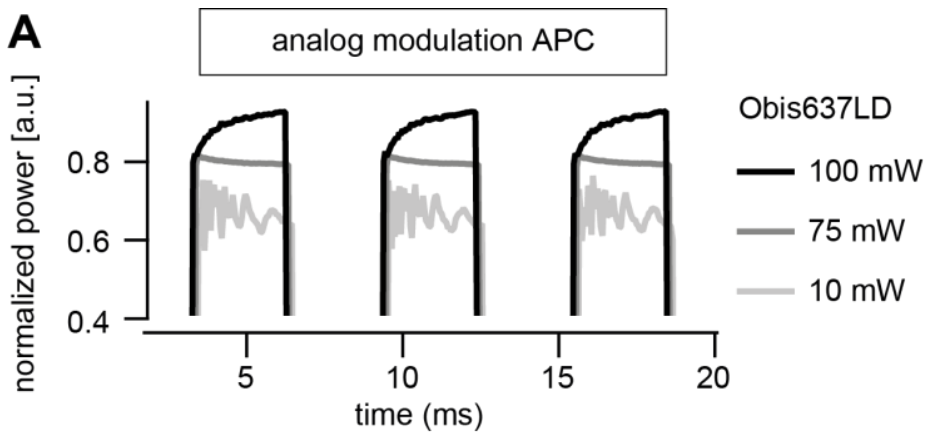

Figure 32: Laser stability using different modes of modulation

[A-C] For the Obis $637 \mathrm{~nm}$ laser, different modulations were tested regarding their stability of the laser output power. A custom made photodiode circuit using a sampling rate of $20 \mathrm{kHz}$ was used to measure the laser power stability for different intensities (10 mW light gray, $75 \mathrm{~mW}$ dark gray, $100 \mathrm{~mW}$ black). Measurements were performed together with Dr. Kai Bodensiek. Laser powers were normalized to their maximum. Three different modulations were tested: [A] analog in constant power (APC) mode, [B] digital in APC mode, [C] digital in constant current (CC) mode. The most stable laser power was obtained for [C] and hence used for voltage imaging experiments.

[D] Fluorescence of an individual TetraSpeck bead illuminated by the Obis 637 $\mathrm{nm}$ laser in the digital modulation CC mode [as in C] at $10 \mathrm{~mW}$ laser intensity was acquired with the sCMOS camera at $1 \mathrm{kHz}$ frame rate. Fluorescence was normalized to the average fluorescence over time $\left(F_{0}\right) ; \Delta F / F_{0}$ scale: 0.1

Compared to the CC mode, the APC mode uses an internal photodiode feedback loop that controls the optical output level. This feedback loop is especially useful, if changes in diode temperature occur, however it can also introduce laser output noise (www.optimaoptics.com/pdf/NOTES.PDF, 05.10.2017). The measured photodiode output indicated that 
the recorded laser powers were only stable in the digitally triggered CC mode, especially for the lowest tested laser intensity of $10 \mathrm{~mW}$ (Figure 32C), which could be due to noise introduced by the photodiode feedback loop used by the APC mode. Hence, the digital CC mode was used during all voltage imaging experiments, later. To avoid heating of the laser diode, the $637 \mathrm{~nm}$ laser was placed on a $2 \mathrm{~cm}$ thick aluminum board for passive cooling. In addition to the photodiode measurements, fluorescence of fixed TetraSpeck beads was acquired with the sCMOS camera ( $1 \mathrm{kHz}$, for $1 \mathrm{~s}$ ) during $10 \mathrm{~mW} 637 \mathrm{~nm}$ laser illumination in the digital CC mode (Figure 32D). Thereby, not only potential laser noise, but also camera noise can be measured. $\Delta F / F_{0}$ of the fluorescent bead signal was calculated the same way as for QuasAr3 fluorescence in SGN boutons, later. Namely, a region of interest (ROI) including a single bead was chosen and $\mathrm{F}_{0}$ was calculated as the average $\mathrm{ROI}$ fluorescence over time. By first subtracting and then dividing the fluorescence of each individual frame by $F_{0}, \Delta F / F_{0}$ was obtained. Fluorescence fluctuations of the bead were in the range of $<0.5$ $\Delta \mathrm{F} / \mathrm{F}_{0}$

\section{Optical fiber stimulation}

In order to employ optical stimulation of Optopatch3-expressing SGN by blue light-induced photocurrents through the channelrhodopsin CheRiff and simultaneously monitor changes in membrane voltage through QuasAr3, a blue light stimulation source had to be installed at the setup. For that, a laser fiber was fixed onto a piezoelectric micromanipulator. The laser fiber brought in close proximity to the organ of Corti is illustrated in Figure 33A. During voltage imaging and optical stimulation, however, the fiber was placed in $250 \mu \mathrm{m}$ distance to SGN boutons (Figure 33A, blue circles), which were placed at the $637 \mathrm{~nm}$ laser illumination area symmetric to the camera center line (see Figure 31C). An analog trigger controlled stimulus timing and blue laser intensity ranging from $0.5 \mathrm{~mW}$ to $16 \mathrm{~mW}$ in the imaging plane (Figure 33B). Laser irradiance at the fiber end in aqueous solution at the location of the sample tissue was calculated based on the irradiation angle of the fiber fixed on the piezoelectric micromanipulator $\left(20^{\circ}\right)$, the distance between the fiber and the sample $(250 \mu \mathrm{m})$, the fiber diameter $(50 \mu \mathrm{m})$ and the numerical aperture of the laser fiber (NA 0.22). The calculated example fiber irradiance profile was plotted for $5 \mathrm{~mW}$ laser output at the fiber exit and shows very high laser irradiance (Figure 33C), exceeding the intensities that were reported previously $\left(0.05\right.$ to $\left.3 \mathrm{~W} / \mathrm{cm}^{2}\right)$ for cell soma stimulation (Hochbaum et al., 2014; Lou et al., 2016). However, the theoretically calculated irradiance was likely overestimated, since the fiber was manually cut and the resulting irradiance angle, thus the loss of power with distance from the fiber, is therefore likely larger. Further, so far, optical CheRiff stimulation was only described for cell bodies (Hochbaum et al., 2014; Lou et al., 2016), which probably require weaker laser irradiance, since more CheRiff channels are present 
and contribute to the depolarizing photocurrent. Hence, in order to assess the minimum laser power required for triggering of APs in SGN boutons, a protocol with increasing laser power steps (intensity from $0.5 \mathrm{~mW}$ to $16 \mathrm{~mW}$, Figure 33B) was employed.

A
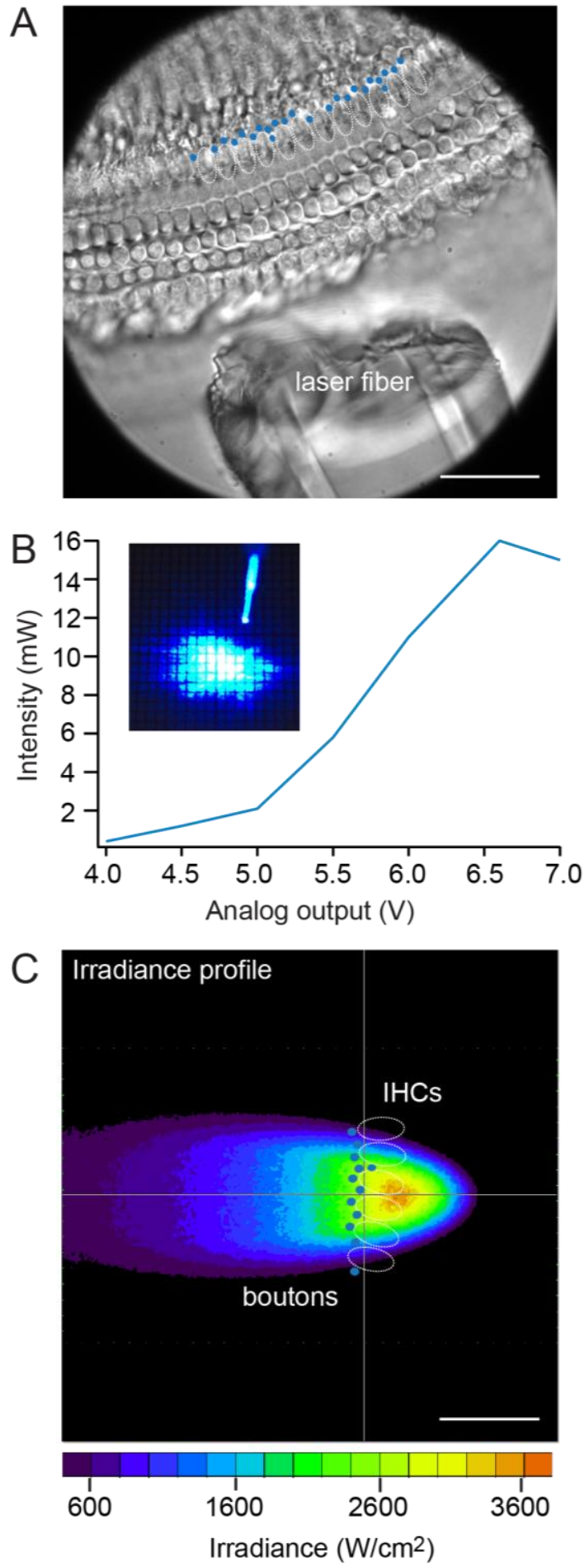

Figure 33: Optical stimulation setup for SGNs with blue light

Installation of a blue light source for optical stimulation of Optopatch3-expressing SGN boutons to induce depolarizing photocurrents through CheRiff.

[A] Bright field image of a freshly dissected organ of Corti with the laser fiber brought in close proximity to the organ of Corti for illustration. Blue circles indicate locations of SGN boutons adjacent to IHCs (white dashed circles). During optical stimulation the laser fiber fixed to a piezoelectric micromanipulator was placed in $250 \mu \mathrm{m}$ distance to the SGNs, which were placed at the $637 \mathrm{~nm}$ laser illumination area symmetric to the camera center line (see Figure 31C). Scale bar: $50 \mu \mathrm{m}$.

[B] Blue laser intensity from a laser fiber was controlled by an analog trigger from a HEKA amplifier (x-axis). The laser fiber output intensity ranged from $0.5 \mathrm{~mW}$ to 16 $\mathrm{mW}$ (y-axis) measured by a photometer in the imaging plane.

[C] Simulation of the fiber irradiance profile at the tissue. The location of the putative position of IHCs and SGN boutons is indicated by white dashed circles and blue circles, respectively. Laser irradiance in aqueous solution at the sample tissue position was calculated based on the irradiation angle of the fiber $\left(20^{\circ}\right)$, the distance between the fiber and the sample $(250 \mu \mathrm{m})$, the fiber diameter $(50 \mu \mathrm{m})$ and the numerical aperture of the laser fiber (NA 0.22). Irradiance lookup table corresponds to $5 \mathrm{~mW}$ laser output at the fiber end. Measurements and calculations were performed together with Dr. Kai Bodensiek. Scale bar: $50 \mu \mathrm{m}$. 
The optical stimulation was first tested in non-virus-transduced SGNs to measure optical crosstalk and thereby investigate proper positioning of the laser fiber to illuminate SGN boutons (Figure 34). Therefore, apical turns of the organ of Corti from non-virus injected mice were freshly dissected and stimulated by $50 \mathrm{~ms}$ pulses of maximal $473 \mathrm{~nm}$ laser intensity ( $6.5 \mathrm{~V}$ analog output, Figure $33 \mathrm{~A})$. Images were acquired with a camera frame rate of $1 \mathrm{kHz}$ and ROls of several SGN boutons were manually chosen for analysis (Figure 34). Normalized fluorescence changes $\left(\Delta F / F_{0}\right)$ of several ROls were plotted over time and show an increase in fluorescence that strongly correlates with the timing of blue light stimulation pulses (Figure 34, dashed box). Hence, the increase in fluorescence likely represents optical crosstalk from the blue laser, which was reported previously (Hochbaum et al., 2014). This experiment indicates that the laser fiber illuminated the organ of Corti at the location of SGN boutons, which is critical for optical SGN stimulation.

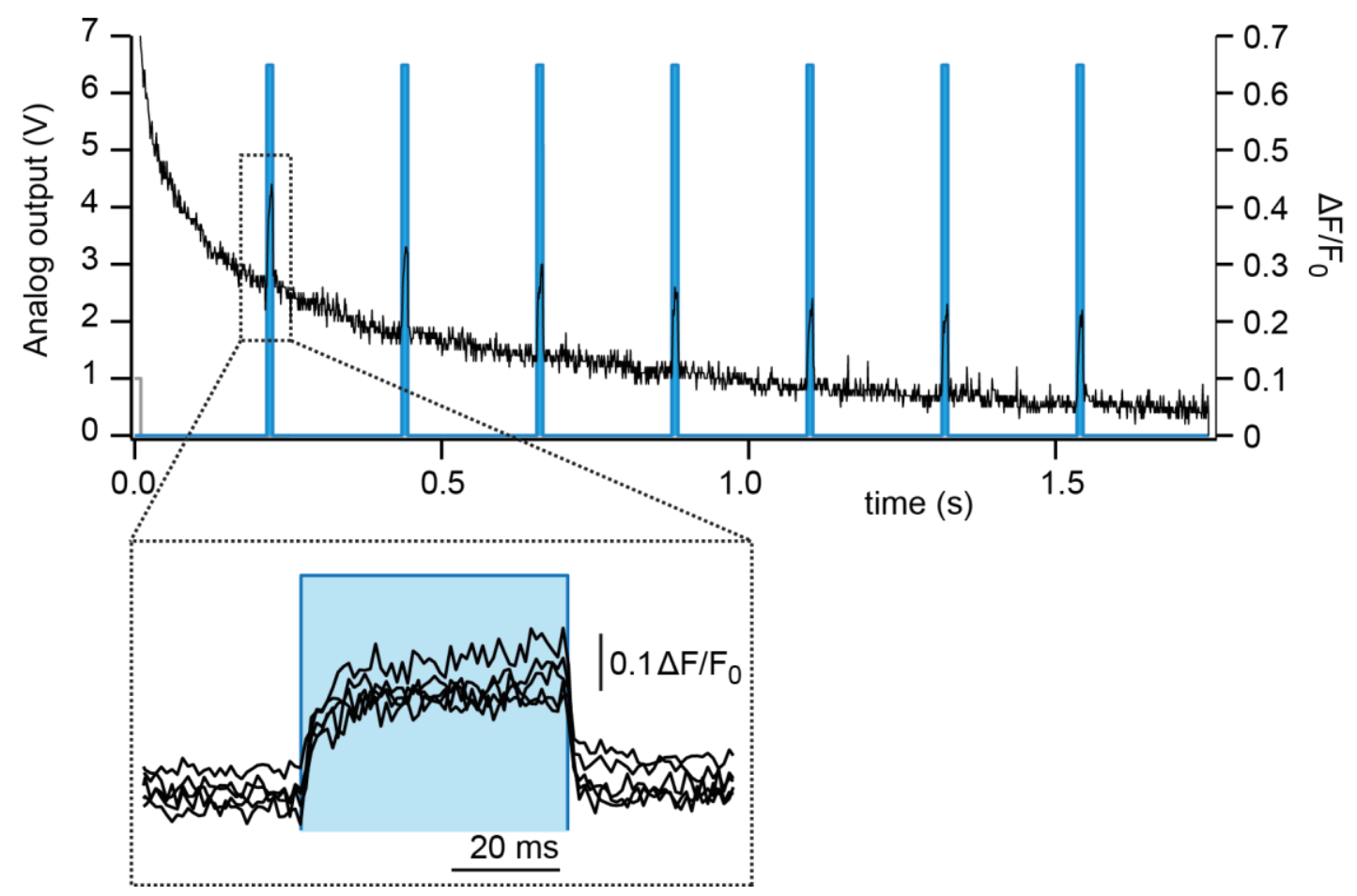

Figure 34: Optical crosstalk by blue light stimulation

$50 \mathrm{~ms}$ pulses of maximal $473 \mathrm{~nm}$ laser intensity (6.5 V analog output, see Figure 32B) illuminated non-virus-transduced SGNs of a freshly dissected organ of Corti. Images were acquired with $1 \mathrm{kHz}$ and $30 \mathrm{~mW} 637 \mathrm{~nm}$ laser intensity. ROls were manually placed on different SGN boutons for analysis. Normalized fluorescence changes $\left(\Delta F / F_{0}\right)$ of several ROls were plotted over time and show an increase in fluorescence that strongly correlates with the timing of blue light stimulation pulses and likely represents optical crosstalk (Hochbaum et al., 2014). 


\section{All-optical electrophysiology in AAV2/6-Optopatch3 transduced mouse cochleae}

Next, optical blue light-stimulation of SGN boutons by CheRiff-mediated photocurrents was combined with optical readout of changes in membrane voltage through QuasAr3. After postnatal virus injection of the cochlea with AAV2/6-Optopatch3, the apical turn of the organ of Corti was dissected and 50 ms pulses of increasing $473 \mathrm{~nm}$ laser intensity were applied to stimulate SGNs (Figure 35). Images were acquired during constant $30 \mathrm{~mW} 637 \mathrm{~nm}$ laser illumination to visualize Quasar3 fluorescence with a camera frame rate of $1 \mathrm{kHz}$. ROls were manually placed on several SGN boutons for analysis (Figure 35A) and normalized fluorescence changes $\left(\Delta F / F_{0}\right)$ of several ROIs from two different IHCs (Figure 35A dashed lines) were plotted over time (Figure 35B) on top of the schematized blue laser stimulation pattern.

A
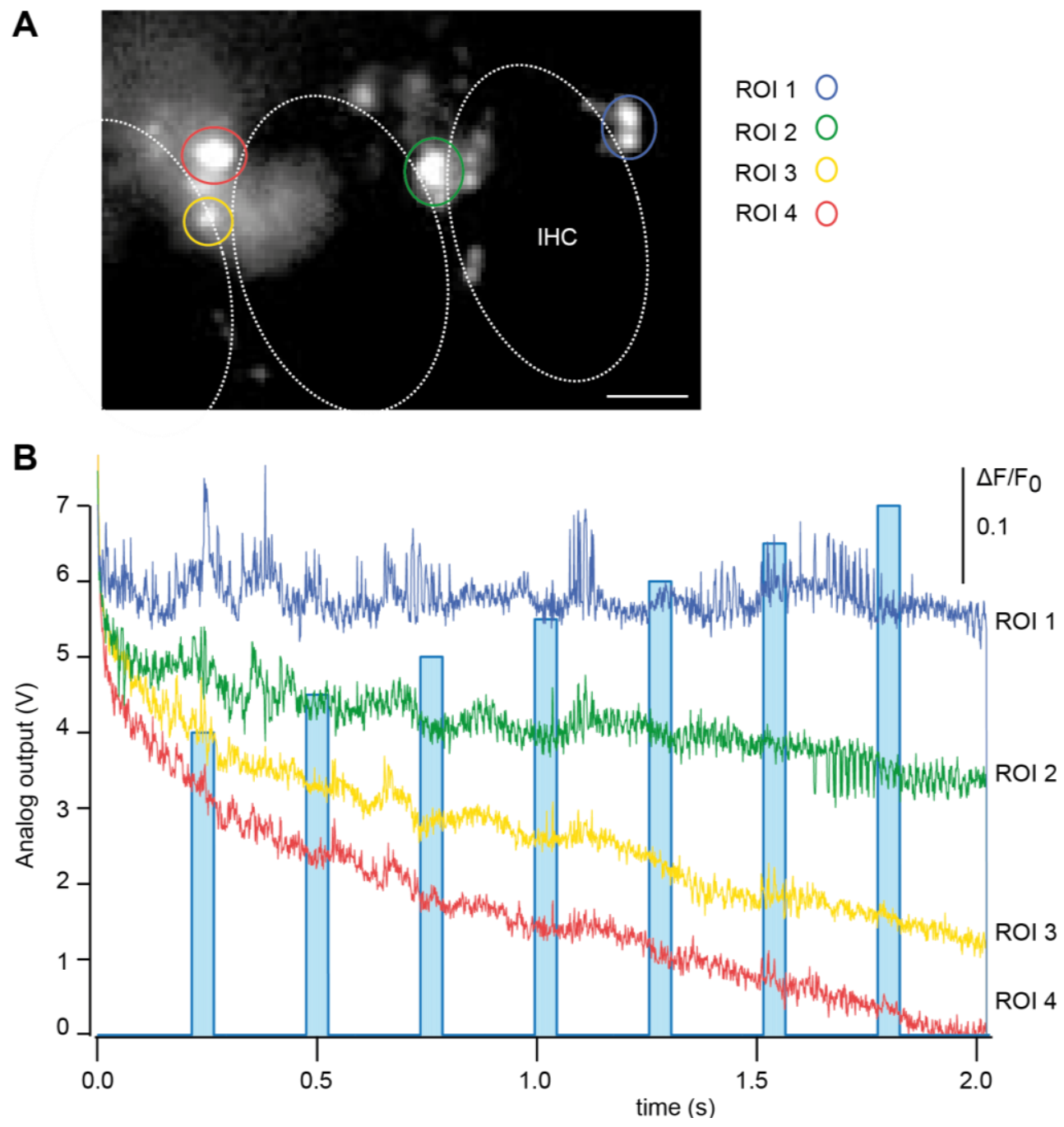

Figure 35: All-optical electrophysiology in SGNs expressing CheRiff and QuasAr3 [A] Fluorescence image of AAV2/6-Optopatch3 transduced SGNs from a freshly dissected organ of Corti. The location of IHCs (dashed white lines) was determined based on a bright field image. SGN boutons (ROI 1-4) adjacent to the IHCs were chosen for image analysis in [B]. Scale bar: $5 \mu \mathrm{m}$. [B] $50 \mathrm{~ms}$ pulses of increasing $473 \mathrm{~nm}$ laser intensity were triggered 
by the HEKA amplifier (analog output, left axis) and are schematized as blue bars. Fluorescence images were acquired with $1 \mathrm{kHz}$ during $30 \mathrm{~mW} 637 \mathrm{~nm}$ laser illumination. Different SGN boutons were chosen manually for analysis as ROls (color code in $[A]$ ). Normalized fluorescence changes $\left(\Delta F / F_{0}\right)$ of $R O I 1$ to 4 were plotted over time. $\Delta F / F_{0}$ traces are displayed with $\mathrm{y}$-offset for better visibility; bar on top right shows $\Delta \mathrm{F} / \mathrm{F}_{0}$ scale of 0.1 .

Fluctuations in the $\Delta F / F_{0}$ traces were most prominent for ROl1 (Figure 35B blue), but the signals did not show obvious correlation with the stimulus pattern. Moreover, optical crosstalk from the blue laser fiber was not as prominent as in the control experiment (Figure 34). Possibly the fiber was not placed in the correct orientation and distance to illuminate the SGN boutons as in the control experiments, such that the induced photocurrents through CheRiff might have been too small to trigger APs in SGNs. Nonetheless, even though the observed $\Delta \mathrm{F} / \mathrm{F}_{0}$ changes were small $(\leq 0.1)$ and did not correlate with the stimulus timing, they might represent APs. As photocurrents could have lowered the SGN spike threshold and thereby increase the probability of spontaneous spike generation, spontaneous secretion of glutamate from IHCs might therefore more easily trigger APs in SGNs. The data interpretation should be treated with caution and does not lead to a final conclusion whether QuasAr3 can be employed as a tool for postsynaptic spike detection in SGNs.

\section{Combining IHC perforated patch-clamp stimulation with QuasAr3 imaging}

Finally, postsynaptic QuasAr3 voltage imaging was combined with presynaptic stimulation of IHCs. Presynaptic IHC patch-clamp stimulation was employed to indirectly stimulate SGNs through exocytic release of glutamate from IHCs and QuasAr3 fluorescence was simultaneously recorded in postsynaptic SGN boutons. Compared to optical stimulation, this approach enables better control and readout of the stimulus, as IHC exocytosis can be monitored by membrane capacitance changes. Further, this stimulus approach is independent of the CheRiff expression level, which determines the amplitude of blue light induced photocurrents and thereby the presence and timing of APs that can be visualized by QuasAr3.

The perforated patch-clamp technique was used to stimulate IHCs from freshly dissected organs of Corti after postnatal virus injection (Figure 36A). Here, whole-cell $\mathrm{Ca}^{2+}$-current $\left(\mathrm{I}_{\mathrm{Ca}}\right)$ and corresponding membrane capacitance $\left(\mathrm{C}_{\mathrm{m}}\right)$ were recorded before, during and after $50 \mathrm{~ms}$ step depolarization of the IHC to $-14 \mathrm{mV}$ (Figure 36B). Even though whole-cell patch clamp recordings integrate the activity of all IHC synapses, the strong depolarization applied here is expected to evoke release at all of the cells synapses. Therefore, the recorded capacitance changes can be used as readout of IHC exocytosis and thus, glutamate release onto postsynaptic SGNs. Simultaneous to the patch-clamp recording, membrane voltage changes of SGNs innervating the IHC were monitored by detecting QuasAr3 fluorescence 
(Figure 36B, bottom). QuasAr3 fluorescence was recorded with $1 \mathrm{kHz}$ camera frame rate under $30 \mathrm{~mW} 637 \mathrm{~nm}$ laser wide field illumination. Based on a merged bright field - and QuasAr3 fluorescence image, the locations of the boutons innervated by the patch-clamped IHC were determined (Figure 36A). ROls including three SGN boutons adjacent to the patch-clamped IHC (Figure 36A,B) and one more distant SGN, which should not be stimulated by the depolarized IHC (Figure 36A,B) were chosen manually for image movie analysis.

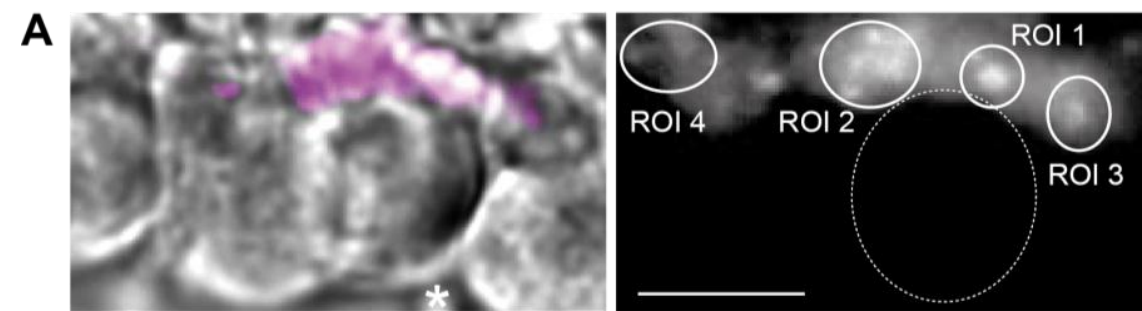

B

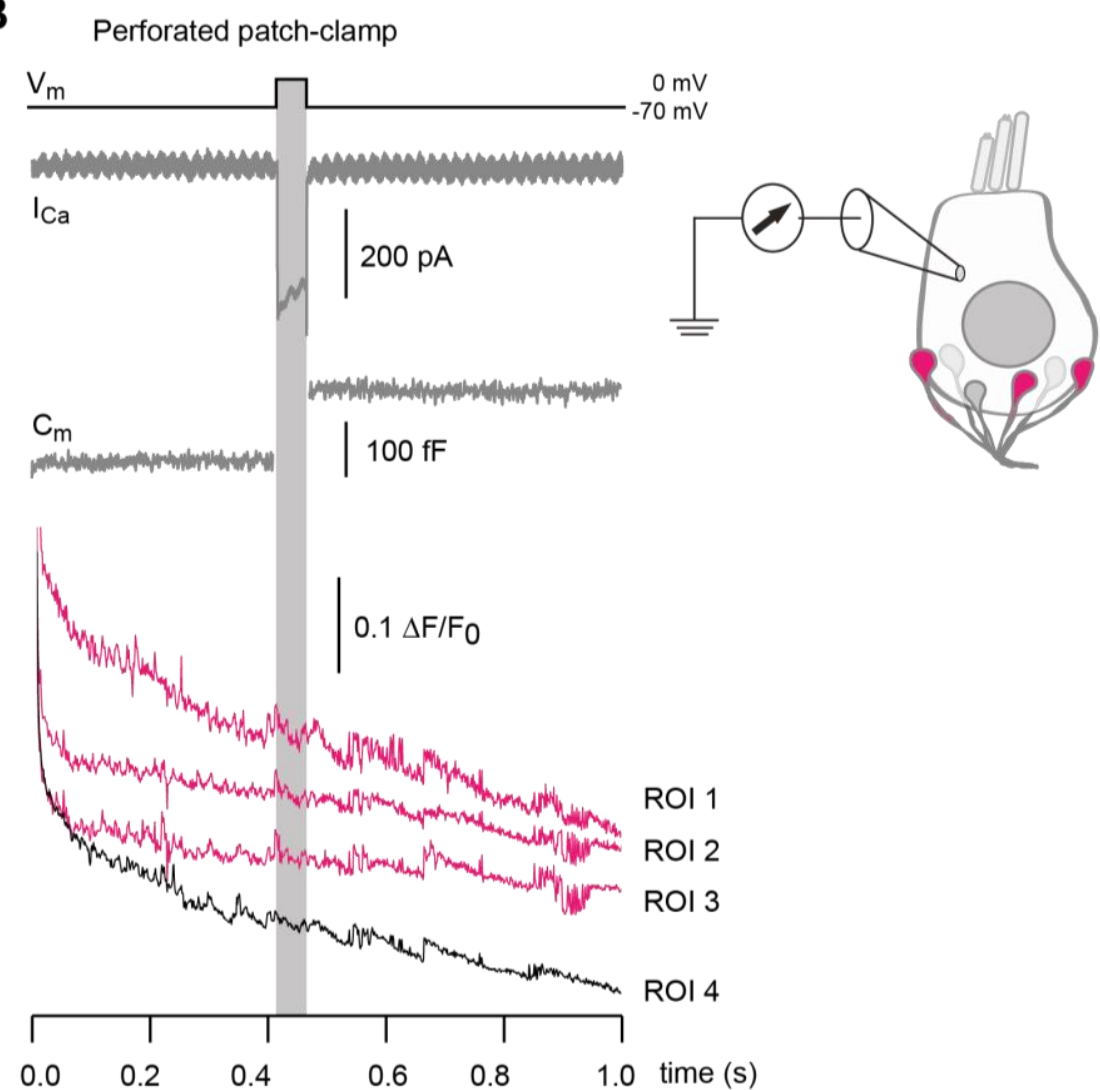

Figure 36: Combining whole-cell patch clamp stimulation of the IHC and postsynaptic QuasAr3 voltage imaging

IHC perforated patch-clamp and simultaneous postsynaptic voltage imaging was performed in a freshly dissected apical turn of an organ of Corti (p14) after postnatal virus injection (p5). [A] Left: Composite of a bright field image (grays) and wide-field $637 \mathrm{~nm}$ illumination fluorescence image (magenta) of the patch-clamped IHC and adjacent QuasAr3 expressing SGN boutons (magenta). Asterisk marks the location of the patch pipette (perforated patchclamp). Right: Wide-field $637 \mathrm{~nm}$ illumination image. The location of the patch-clamped IHC is indicated by a dashed white circle. ROls were manually chosen at the location of SGN boutons adjacent to the patch-clamped IHC (ROI 1-3) and adjacent to a non-patch-clamped 
IHC (ROI 4) for image analysis in [B] (white circles). Scale bar: $10 \mu \mathrm{m}$. [B] Whole-cell perforated patch-clamp recording of an IHC (gray traces) and simultaneously recorded QuasAr3 fluorescence from SGN boutons adjacent to the patch-clamped IHC (pink and black traces). Whole-cell Ca ${ }^{2+}$-current (I $\mathrm{Ca}$, gray) and corresponding membrane capacitance $\left(\mathrm{C}_{\mathrm{m}}\right.$, gray) of an IHC before, during and after $50 \mathrm{~ms}$ step depolarization to $-14 \mathrm{mV}$ (black line and gray bar). Pink traces show simultaneously recorded normalized QuasAr3 fluorescence changes $(\triangle \mathrm{F} / \mathrm{F} 0)$ corresponding to $\mathrm{ROI} 1-3$ adjacent to the IHC (illustrated in $[A])$. Black trace shows the QuasAr3 $\Delta$ F/F0 signal corresponding to ROI 4 distant to the patch-clamped IHC (illustrated in [A]). Traces are displayed with y-offset for better visibility. Images were acquired at $1 \mathrm{kHz}$ camera frame rate and $30 \mathrm{~mW} 637 \mathrm{~nm}$ laser power. $\Delta F / F 0$ scale: 0.1 .

QuasAr3 fluorescence for each ROI was normalized as explained above. Pink traces show simultaneously recorded normalized QuasAr3 $\Delta F / F_{0}$ signals corresponding to ROls 1-3 adjacent to the IHC, whereas the black trace shows the QuasAr3 $\Delta \mathrm{F} / \mathrm{F}_{0}$ signal corresponding to ROI 4, distant from the patch-clamped IHC (illustrated in Figure 36A). Even though a slight increase in $\Delta F / F_{0}$ QuasAr3 signal was visible at the onset of the stimulus in ROI 1-3, the amplitude was not larger than the fluctuations before and after IHC stimulation. Since the IHC was voltage clamped at $-70 \mathrm{mV}$, no spontaneous SV release and thus postsynaptic spiking would be expected. Hence, also this experiment was not conclusive on whether or not QuasAr3 could be employed as a tool for postsynaptic spike detection in SGNs.

\subsection{Discussion}

Sound-evoked glutamate release from an IHC synapse is sensed by the postsynaptic SGNs that connect the peripheral sensory sound receptor system to the brain stem. While each IHC innervates 5-30 SGNs, each SGN is innervated by only one ribbon-type synapse. And even though all IHC AZs are driven by the same IHC receptor membrane potential, postsynaptic spike responses of SGNs differ remarkably. There is emerging evidence that postsynaptic response diversity is achieved by the heterogeneity of presynaptic AZs. Livecell confocal $\mathrm{Ca}^{2+}$-imaging revealed that larger ribbons clustered more synaptic $\mathrm{Ca}^{2+}$ channels, showed increased synaptic $\mathrm{Ca}^{2+}$-influx, and were located towards the modiolar side of the IHC. Those AZs further displayed a more depolarized $\mathrm{V}_{\text {half }}$ of $\mathrm{Ca}^{2+}$-influx (Frank et al., 2009; Meyer et al., 2009; Ohn et al., 2016). However, evidence for heterogeneous SGN responses regarding spontaneous spike rate, spike threshold, and dynamic spike range for SGNs with similar characteristic frequency is mostly based on in vivo extracellular recordings from ANFs (Liberman, 1978; Ohlemiller and Echteler, 1990; Sachs and Abbas, 1974; Taberner and Liberman, 2005; Yates et al., 1990). When examining cat SGNs, it was possible to morphologically correlate the preferential innervation side of the $\mathrm{IHC}$ with the 
type of firing pattern (low/medium-SR and high-SR SGNs) of the SGN (Kawase and Liberman, 1992; Liberman, 1980, 1982; Liberman and Oliver, 1984). However, so far, there is no physiological evidence that proves a direct correlation pattern between the diverse above-mentioned IHC AZ properties and postsynaptic responses. To provide mechanistic insights into synaptic sound encoding, imaging techniques that monitor the activity of individual presynaptic $\mathrm{AZs}$ and SGN boutons are promising tools. Confocal $\mathrm{Ca}^{2+}$-imaging of individual presynaptic AZs is well established in IHCs (Frank et al., 2009; Meyer et al., 2009; Ohn et al., 2016) and can be combined with the use of a fluorescently labelled RIBEYEbinding peptide to mark presynaptic AZs (Francis et al., 2011). However, to date there is no established postsynaptic readout system for monitoring multiple individual SGN responses. Compared to $\mathrm{Ca}^{2+}$-indicators, voltage indicators are superior regarding their high-speed event kinetics and therefore most suitable to monitor neuronal APs. Both QuasAr1 and QuasAr2 could resolve individual APs in hippocampal neurons in single trials and have redshifted fluorescence spectra (Hochbaum et al., 2014), which would allow imaging in combination with green fluorescent presynaptic $\mathrm{Ca}^{2+}$-indicators in the future. Therefore, I established an experimental setup for live-cell voltage imaging (Figure 10 and 32-32) of QuasAr variants in SGNs. While QuasAr2 imaging was performed in a Floxpatch-Bhlhb5Cre (Lou et al., 2016; Ross et al., 2010) mouse line (in the laboratory of Prof. Adam Cohen) using direct optical blue-light SGN stimulation to induce depolarizing photocurrents through the co-expressed channelrhodopsin CheRiff, QuasAr3 imaging was performed in postnatally virus-injected mice using either direct optical stimulation or indirect SGN stimulation through patch-clamp stimulation of presynaptic IHCs. Regardless of the expression system, the GEVIs QuasAr2 and 3 were both expressed in cochlear SGNs (Figure 26 and 28) and high levels of QuasAr3 were detected in SGN boutons adjacent to IHCs (Figure 29).

However, during imaging experiments only QuasAr3 (Figure 35, 36), but not QuasAr2 (Figure 27), elicited any fluorescence responses in SGN boutons adjacent to an IHC. QuasAr3 carries the same point mutation Q95H as QuasAr1, which was previously reported to have extremely fast fluorescent on- and off-kinetics of $<1 \mathrm{~ms}$, but a lower sensitivity to membrane voltage-changes (Hochbaum et al., 2014). On the other hand, QuasAr2 is more sensitive to membrane voltage-changes, while its on- and off-kinetics are in the range of 1 ms (Hochbaum et al., 2014). The QuasAr2 response kinetics to voltage changes is still superior to other indicators like ArcLight (Piao et al., 2015), but might be still too slow to resolve APs of SGNs, since the FWHM of APs in SGNs was previously reported to be on average $0.9 \mathrm{~ms}$ (Rutherford et al., 2012). Hence, one could speculate that in this work, no APs could be detected in SGNs by the use of QuasAr2, because the fluorescence change 
in this short depolarized period might have been too small due to the "slow" on-kinetics ( $\tau_{\text {on }} 1.2 \mathrm{~ms}$ ) of QuasAr2 (Hochbaum et al., 2014). Alternatively, or additionally, a complication in the experiments could as well be a relatively lower expression level of QuasAr2 in the transgenic Floxpatch mouse compared to virus-mediated expression in QuasAr3 experiments. Nonetheless, the CAG promoter employed in the Floxpatch mouse is a ubiquitous promoter (Lou et al., 2016) and was previously reported to achieve high transcription levels in the Rosa26 locus (Chen et al., 2011).

Nonetheless, the slight increase in $\Delta \mathrm{F} / \mathrm{F}_{0}$ signal amplitude for QuasAr3 during optical stimulation of SGNs or electrical stimulation of IHCs was not larger than the QuasAr3 signal fluctuations before and after the stimuli and comparable or only slightly bigger than the noise floor measured by the fluorescence beads (Figure 32). In addition, in the IHC patch-clamp experiment, not only the SGNs adjacent to the stimulated IHC (ROI 1-3), but also the more distant SGN (ROI 4) showed temporally correlated $\Delta F / F_{0}$ signal fluctuations (Figure 36), which argues against the detection of IHC triggered APs in this particular experiment. Moreover, during the patch-clamp experiment, the $\mathrm{IHC}$ was voltage clamped at $-70 \mathrm{mV}$, where no spontaneous SV release and thus postsynaptic spiking would be expected. Therefore, it is hard to distinguish between the desired QuasAr3 responses to membrane voltage changes or noise. To investigate whether QuasAr3 signals are indeed caused by evoked APs, a control experiment by application of tetrodotoxin (TTX), a Nav channel blocker that inhibits the generation of APs, could be performed. At this point of the project, however, it is not possible yet to draw a conclusion on whether QuasAr3 can be used to monitor APs in SGNs and probe the presynaptic mechanism of postsynaptic response diversity.

Overall, the sensitivity of the wide-field imaging setup could be further increased in order to achieve robust fluorescence QuasAr3 responses, which are reliably distinguishable from background noise. Voltage imaging is a balancing act between signal detection and speed. While extremely fast on- and off- kinetics of the voltage indicator and high-speed acquisition by the camera are required to monitor APs, the signal-to-noise ratio (SNR) decreases under high-speed conditions. Technically, an increase in the SNR could be achieved by a higher NA objective (currently 60x, NA 0.9) to capture as many photons as possible. On the other hand, the choice of the objective is limited by the required large working distance, since for IHC patch-clamp recordings, an upright microscope with a water-dipping objective is most suitable. Further, the SNR could be increased by a low camera readout noise, and a high quantum efficiency of the camera chip in the far-red imaging range $(>700 \mathrm{~nm})$. However, voltage imaging also requires fast acquisition of a reasonable area (at least $15 \times 15 \mu \mathrm{m}$ to 
enable recording from one IHC with several adjacent boutons) with reasonable resolution (approximately $200 \mathrm{~nm}$ to distinguish individual boutons).

Aside from signal-to-noise issues, there is another caveat regarding the high-speed kinetics of voltage indicator fluorescence. Since the number of photons that are emitted by the sensor and detected by the camera per time and pixel gets highly reduced especially during high-speed recordings where the exposure time is extremely short ( $\leq 1 \mathrm{~ms})$, thus requiring higher illumination intensities for adequate signal amplitudes. Thereby, the brightness and quantum yield of the indicator becomes very important. Rhodopsin based voltage indicators have a small quantum yield $(<0.001)$ and require high irradiance because of their complex photo cycle (Hochbaum et al., 2014; Maclaurin et al., 2013). Even though Arch variants are robust to photobleaching (Kralj et al., 2011), the high irradiance at the imaged tissue might induce thermal damage. This was however not observed in this study. During simultaneous IHC patch-clamp recording and QuasAr3 imaging in SGNs, patch-clamped IHCs were repetitively irradiated with approximately $1000 \mathrm{~W} / \mathrm{cm}^{2} 637 \mathrm{~nm}$ laser light for $1 \mathrm{~s}$ and showed stable $\mathrm{Ca}^{2+}$-currents and exocytosis. SGN boutons however have a much smaller volume compared to IHCs, whereby thermal tissue heating might have a different effect.

If optimization of the wide-field imaging setup through an increase in sensitivity and thereby more reliable optical detection of APs might not lead to the desired outcome, one could think about alternative GEVIs. A good alternative to rhodopsin-based voltage indicators are e.g. FRET-based voltage indicators, such as the recently developed blue-shifted AcemNeon versions (Gong et al., 2015). The FRET pair consists of an opsin with the bright and photostable fluorescent protein mNeonGreen (Gong et al., 2015). The fast kinetics $(<0.5$ ms) of Ace-mNeon allowed single-cell and single-AP resolution in mouse and fly brains in vivo (Gong et al., 2015). Unfortunately, the fluorescent spectrum of Ace-mNeon is blueshifted, which would not allow simultaneous $\mathrm{Ca}^{2+}$-imaging in presynaptic IHC AZs using the well-established small molecule green $\mathrm{Ca}^{2+}$-indicators Fluo-4FF or Fluo-5N (Frank et al., 2009; Meyer et al., 2009). Combination of pre- and postsynaptic imaging would provide a functional link between heterogeneous presynaptic AZ properties and postsynaptic SGN spike response diversity. Nonetheless, one could use one of the less well-established red fluorescent $\mathrm{Ca}^{2+}$-indicators or at least make use of the fluorescently labelled RIBEYEbinding peptide (Francis et al., 2011) to mark presynaptic AZs and estimate the presynaptic ribbon size as measure for AZ strength (Frank et al., 2009). Since AAV2/6 mediated gene expression after postnatal virus injection into the round window of the cochlea worked well, this method could be applied to other genetically encoded sensors such as Ace-mNeon. 


\section{Outlook}

To understand synaptic transmission, it is essential that we identify and characterize the molecular components involved in it and their role in the functional molecular networks mediating this process. One obvious crucial step in synaptic transmission is exocytosis, but in addition, we will have to understand the post-synaptic processes that are responsible for decoding and transmitting the presynaptic output better. Finally, we will have to combine the synaptic physiology with computational methods to produce a systems relevant model for processing of sound information. This is possible by examining auditory synaptic information processing on the molecular, anatomical, physiological, and systems level, as it provides knowledge about the molecular key players in synaptic transmission and about how auditory information processing works along the auditory pathway.

There is evidence that IHCs decompose auditory information such as sound pressure intensity through their heterogeneous synaptic properties. This enables them to forward already decomposed information along separate neuronal trajectories towards the brain. Using presynaptic $\mathrm{Ca}^{2+}$-imaging and postsynaptic single unit ANF recordings, Gipc3 was identified as a presynaptic candidate protein for modulating the activation of presynaptic $\mathrm{Ca}^{2+-i n f l u x}$ and thereby modulating the spontaneous SGN firing rate (Ohn et al., 2016). Genetic deletion of Gipc3 in mice shifted the $\mathrm{Ca}^{2+}$-channel $\mathrm{V}_{\text {half }}$ to more hyperpolarized potentials, thereby increasing IHC exocytosis and spontaneous SGN discharge rates (Ohn et al., 2016). In addition, the gradient of $\mathrm{Ca}^{2+}$-channel $\mathrm{V}_{\text {half }}$ and maximal $\mathrm{Ca}^{2+}$-influx along the IHC symmetry axis was reversed compared to wild-type AZs (Ohn et al., 2016). Despite Gipc3, the molecular players allowing for such heterogeneous IHC synapse properties have, however, not been identified yet. Voltage imaging in SGNs that receive input from a single IHC will provide novel insights into the mechanism of how IHCs decompose stimulatory input via their multiple heterogeneous AZs and how SGNs respond to it. Hence, SGN voltage imaging might be a useful tool to screen for new candidate molecules modulating presynaptic synapse heterogeneity and change postsynaptic SGN response properties. Since AAV-mediated expression of GEVIs makes this technique applicable to any transgenic candidate mouse line, it may allow us to investigate whether synapse heterogeneity and postsynaptic responses might be altered in different knockout mice. Even though, the QuasAr3 imaging experiments presented in this work are not yet conclusive on whether this particular GEVI is suitable to detect APs in SGNs, voltage imaging using GEVIs and AAV2/6 mediated gene expression in SGNs has a great potential. Voltage imaging is technically very challenging and constitutes a balancing act between acquisition speed, 
fluorescence signal detection and noise. Further, high GEVI expression levels and their correct plasma membrane localization are required. Nonetheless, voltage imaging is a cutting-edge technique and once, it is fully established, will provide novel insights into synaptic sound coding strategies through its high temporal resolution.

Towards the overall goal to identify and characterize novel molecular components involved in IHC exocytosis, I found that RIM-BP2 plays a key role at IHC AZs. By studying the morphological and physiological effects of genetic RIM-BP2 disruption in constitutive RIM$B P 2$ knockout mice, I found that in murine IHCs RIM-BP2 (1) positively regulates the number of synaptic Cav1.3 $\mathrm{Ca}^{2+}$-channels (Figure 14, 15) and thereby facilitates SV release (Figure 16), and (2) enhances fast SV recruitment after RRP depletion (Figure 18, 24). This was physiologically reflected by a mild auditory synaptopathy. The elevated hearing thresholds and reduced $A B R$ wave I amplitude in combination with normal otoacoustic emissions in RIM-BP2 knockout mice (Figure 21) are also often found in human patients with impaired speech comprehension (Moser et al., 2006b). Hence, the question arises, whether humans also express RIM-BP2 and whether it is associated with human hearing loss. Interestingly, the human gene for RIM-BP2 was mapped to the human chromosome 12q24.33 (Yan et al., 2005), which was previously defined as the gene locus of DFNA41, an autosomal dominant sensorineural hearing loss that is progressive and usually starts in the second decade of life (Blanton et al., 2002). Furthermore, the mouse and human RIMBP2 amino acid sequences share 83\% identity (NCBI Blast (Altschul et al., 1997) result of NP_056162.4 [homo sapiens] versus NP_001074857.1 [mus musculus]). Hence, both, the gene location of RIM-BP2 in the DFNA41 locus and the high amino acid sequence homology between human and mouse RIM-BP2 suggest that indeed RIM-BP2 might play a role in IHC synaptic transmission in humans and that it might be a relevant candidate affected by the human hereditary hearing loss. Indeed, RIM-BP2 was indeed found to be expressed by human IHCs (Girotto et al., 2014). Surprisingly however, a RIM-BP2 mutation in humans caused a reduction in patients' hearing thresholds (Girotto et al., 2014), which stands in contrast to the elevated hearing thresholds found in the RIM-BP2 knockout mouse model (Figure 21). Hence, more in depth analysis is required e.g. to map and pinpoint the affected protein sequence location and domain of the human RIM-BP2 mutation. As a next step, one could generate and characterize a mouse model carrying this human RIM-BP2 mutation. Thereby one could relate the findings from mice to humans and learn more about the molecular function of RIM-BP2 in IHC synaptic transmission. Finally, gene therapy might be employed as a future perspective to restore hereditary hearing loss in humans using virus mediated transgenic expression of deafness genes (reviewed in: Ahmed et al., 2017). 
Previously, gene replacement successfully restored hearing in deaf mice lacking the vesicular glutamate transporter vGlut3 or adaptor protein 2 (Akil et al., 2012; Jung et al., 2015b).

Finally, it will be interesting to explore the function of the other RIM-BP isoforms (RIM-BP1 and RIM-BP3) in knockout mouse models. I collected preliminary data from a RIM-BP1 and RIM-BP2 double-knockout (RIM-BP1/2 DKO) mouse line (Grauel et al., 2016) by measuring presynaptic $\mathrm{Ca}^{2+}$-influx and exocytic capacitance changes during IHC perforated patchclamp recordings and ABRs (by Nadine Dietrich). Surprisingly, both the presynaptic $\mathrm{Ca}^{2+}$ influx and exocytosis were increased compared to RIM-BP2 single-knockout IHCs, whereas ABR thresholds were further elevated. Hence, RIM-BP1 might turn out to be an attractive new candidate to further disentangle the unconventional synaptic machinery of IHCs. 


\section{References}

Acuna, C., Liu, X., Gonzalez, A., and Südhof, T.C. (2015). RIM-BPs Mediate Tight Coupling of Action Potentials to Ca2+-Triggered Neurotransmitter Release. Neuron 87, 1234-1247.

Acuna, C., Liu, X., and Südhof, T.C. (2016). How to Make an Active Zone: Unexpected Universal Functional Redundancy between RIMs and RIM-BPs. Neuron 91, 792807.

Ahmed, H., Shubina-Oleinik, O., and Holt, J.R. (2017). Emerging Gene Therapies for Genetic Hearing Loss. J. Assoc. Res. Otolaryngol. 18, 649-670.

Akil, O., Seal, R.P., Burke, K., Wang, C., Alemi, A., During, M., Edwards, R.H., and Lustig, L.R. (2012). Restoration of Hearing in the VGLUT3 Knockout Mouse Using Virally Mediated Gene Therapy. Neuron 75, 283-293.

Almers, W., Zenisek, D., and Steyer, J.A. (2000). Transport, capture and exocytosis of single synaptic vesicles at active zones. Nature 406, 849-854.

Altschul, S.F., Madden, T.L., Schäffer, A.A., Zhang, J., Zhang, Z., Miller, W., and Lipman, D.J. (1997). Gapped BLAST and PSI-BLAST: a new generation of protein database search programs. Nucleic Acids Res. 25, 3389-3402.

Andrews-Zwilling, Y.S., Kawabe, H., Reim, K., Varoqueaux, F., and Brose, N. (2006). Binding to Rab3A-interacting molecule RIM regulates the presynaptic recruitment of Munc13-1 and ubMunc13-2. J. Biol. Chem. 281, 19720-19731.

Ashmore, J. (2008). Cochlear Outer Hair Cell Motility. Physiol. Rev. 88, 173-210.

Ashmore, J., Avan, P., Brownell, W.E., Dallos, P., Dierkes, K., Fettiplace, R., Grosh, K., Hackney, C.M., Hudspeth, A.J., Jülicher, F., et al. (2010). The remarkable cochlear amplifier. Hear. Res. 266, 1-17.

Assad, J.A., Shepherd, G.M.G., and Corey, D.P. (1991). Tip-link integrity and mechanical transduction in vertebrate hair cells. Neuron 7, 985-994.

Augustine, G.J., Adler, E.M., and Charlton, M.P. (1991). The calcium signal for transmitter secretion from presynaptic nerve terminals. Ann. N. Y. Acad. Sci. 635, 365-381.

Baig, S.M., Koschak, A., Lieb, A., Gebhart, M., Dafinger, C., Nürnberg, G., Ali, A., Ahmad, I., Sinnegger-Brauns, M.J., Brandt, N., et al. (2011). Loss of Cav1.3 (CACNA1D) function in a human channelopathy with bradycardia and congenital deafness. Nat. Neurosci. 14, 77-84.

Bartoletti, T.M., Jackman, S.L., Babai, N., Mercer, A.J., Kramer, R.H., and Thoreson, W.B. (2011). Release from the cone ribbon synapse under bright light conditions can be controlled by the opening of only a few $\mathrm{Ca}(2+)$ channels. J Neurophysiol 106, 2922-2935.

Baumann, L., Gerstner, A., Zong, X., Biel, M., and Wahl-Schott, C. (2004). Functional Characterization of the L-type Ca2+ Channel Cav1.4a1 from Mouse Retina. Investig. Ophthalmol. Vis. Sci. 45, 708-713.

Bech-Hansen, N.T., Naylor, M.J., Maybaum, T.A., Pearce, W.G., Koop, B., Fishman, G.A., Mets, M., Musarella, M.A., and Boycott, K.M. (1998). Loss-of-function mutations in a calcium-channel alpha1-subunit gene in Xp11.23 cause incomplete X-linked congenital stationary night blindness. Nat. Genet. 19, 264-267.

Beurg, M., Evans, M.G., Hackney, C.M., and Fettiplace, R. (2006). A LargeConductance Calcium-Selective Mechanotransducer Channel in Mammalian 
Cochlear Hair Cells. J. Neurosci. 26, 10992-11000.

Beutner, D., and Moser, T. (2001). The presynaptic function of mouse cochlear inner hair cells during development of hearing. J. Neurosci. 21, 4593-4599.

Beutner, D., Voets, T., Neher, E., and Moser, T. (2001). Calcium dependence of exocytosis and endocytosis at the cochlear inner hair cell afferent synapse. Neuron 29, 681-690.

Bharadwaj, H.M., Verhulst, S., Shaheen, L., Liberman, M.C., and Shinn-Cunningham, B.G. (2014). Cochlear neuropathy and the coding of supra-threshold sound. Front. Syst. Neurosci. 8, 26.

Blanton, S.H., Liang, C.Y., Cai, M.W., Pandya, A., Du, L.L., Landa, B., Mummalanni, S., Li, K.S., Chen, Z.Y., Qin, X.N., et al. (2002). A novel locus for autosomal dominant non-syndromic deafness (DFNA41) maps to chromosome 12q24-qter. J. Med. Genet. 39, 567-570.

Borst, J.G.G., and Sakmann, B. (1996). Calcium influx and transmitter release in a fast CNS synapse. Nature 383, 431-434.

Brandt, A., Striessnig, J., and Moser, T. (2003). CaV1.3 channels are essential for development and presynaptic activity of cochlear inner hair cells. J. Neurosci. 23, 10832-10840.

Brandt, A., Khimich, D., and Moser, T. (2005). Few CaV1.3 Channels Regulate the Exocytosis of a Synaptic Vesicle at the Hair Cell Ribbon Synapse. J. Neurosci. 25, 11577-11585.

Bunt, A.H. (1971). Enzymatic digestion of synaptic ribbons in amphibian retinal photoreceptors. Brain Res. 25, 571-577.

Buran, B.N., Strenzke, N., Neef, A., Gundelfinger, E.D., Moser, T., and Liberman, M.C. (2010). Onset Coding Is Degraded in Auditory Nerve Fibers from Mutant Mice Lacking Synaptic Ribbons. J. Neurosci. 30, 7587-7597.

Chapochnikov, N.M., Takago, H., Huang, C.H., Pangršič, T., Khimich, D., Neef, J., Auge, E., Göttfert, F., Hell, S.W., Wichmann, C., et al. (2014). Uniquantal release through a dynamic fusion pore is a candidate mechanism of hair cell exocytosis. Neuron 83, 1389-1403.

Chen, C., Krohn, J., Bhattacharya, S., and Davies, B. (2011). A comparison of exogenous promoter activity at the ROSA26 locus using a ФiC31 integrase mediated cassette exchange approach in mouse ES cells. PLoS One 6, e23376.

Chen, Z., Kujawa, S.G., and Sewell, W.F. (2007). Auditory sensitivity regulation via rapid changes in expression of surface AMPA receptors. Nat. Neurosci. 10, 12381240.

Cho, S., Li, G.-L., and von Gersdorff, H. (2011). Recovery from Short-Term Depression and Facilitation Is Ultrafast and Ca2+ Dependent at Auditory Hair Cell Synapses. J. Neurosci. 31, 5682-5692.

Cole, A.A., Chen, X., and Reese, T.S. (2016). A Network of Three Types of Filaments Organizes Synaptic Vesicles for Storage, Mobilization, and Docking. J. Neurosci. 36, 3222-3230.

Coppola, T., Magnin-Lüthi, S., Perret-Menoud, V., Gattesco, S., Schiavo, G., and Regazzi, R. (2001). Direct Interaction of the Rab3 Effector RIM with Ca2+ Channels, SNAP-25, and Synaptotagmin. J. Biol. Chem. 276, 32756-32762.

Corey, D.P., and Hudspeth, A.J. (1983). Kinetics of the receptor current in bullfrog saccular hair cells. J. Neurosci. 3, 962-976. 
Davydova, D., Marini, C., King, C., Klueva, J., Bischof, F., Romorini, S., MontenegroVenegas, C., Heine, M., Schneider, R., Schröder, M.S., et al. (2014). Bassoon specifically controls presynaptic P/Q-type $\mathrm{Ca} 2+$ channels via RIM-binding protein. Neuron 82, 181-194.

Dick, O., Tom Dieck, S., Altrock, W.D., Ammermüller, J., Weiler, R., Garner, C.C., Gundelfinger, E.D., and Brandstätter, J.H. (2003). The presynaptic active zone protein bassoon is essential for photoreceptor ribbon synapse formation in the retina. Neuron 37, 775-786.

Dou, H., Vazquez, A.E., Namkung, Y., Chu, H., Cardell, E. Lou, Nie, L., Parson, S., Shin, H.-S., and Yamoah, E.N. (2004). Null mutation of alpha1D Ca2+ channel gene results in deafness but no vestibular defect in mice. J. Assoc. Res. Otolaryngol. 5, 215-226.

Dulubova, I., Lou, X., Lu, J., Huryeva, I., Alam, A., Schneggenburger, R., Südhof, T.C., and Rizo, J. (2005). A Munc13/RIM/Rab3 tripartite complex: from priming to plasticity? EMBO J. 24, 2839-2850.

Edmonds, B.W., Gregory, F.D., and Schweizer, F.E. (2004). Evidence that fast exocytosis can be predominantly mediated by vesicles not docked at active zones in frog saccular hair cells. J. Physiol. 560, 439-450.

Eggermann, E., Bucurenciu, I., Goswami, S.P., and Jonas, P. (2011). Nanodomain coupling between $\mathrm{Ca} 2+$ channels and sensors of exocytosis at fast mammalian synapses. Nat. Rev. Neurosci. 13, 7-21.

Fedchyshyn, M.J. (2005). Developmental Transformation of the Release Modality at the Calyx of Held Synapse. J. Neurosci. 25, 4131-4140.

Fenster, S.D., Chung, W.J., Zhai, R., Cases-Langhoff, C., Voss, B., Garner, a M., Kaempf, U., Kindler, S., Gundelfinger, E.D., and Garner, C.C. (2000). Piccolo, a presynaptic zinc finger protein structurally related to bassoon. Neuron 25, 203-214.

Fettiplace, R. (2016). Is TMC1 the Hair Cell Mechanotransducer Channel? Biophys. J. 111, 3-9.

Fettiplace, R., and Kim, K.X. (2014). The physiology of mechanoelectrical transduction channels in hearing. Physiol. Rev. 94, 951-986.

Fouquet, W., Owald, D., Wichmann, C., Mertel, S., Depner, H., Dyba, M., Hallermann, S., Kittel, R.J., Eimer, S., and Sigrist, S.J. (2009). Maturation of active zone assembly by Drosophila Bruchpilot. J. Cell Biol. 186, 129-145.

Francis, A.A., Mehta, B., and Zenisek, D. (2011). Development of new peptide-based tools for studying synaptic ribbon function. J. Neurophysiol. 106, 1028-1037.

Frank, T., Khimich, D., Neef, A., and Moser, T. (2009). Mechanisms contributing to synaptic $\mathrm{Ca} 2+$ signals and their heterogeneity in hair cells. Proc. Natl. Acad. Sci. U. S. A. $106,4483-4488$.

Frank, T., Rutherford, M.A., Strenzke, N., Neef, A., Pangršič, T., Khimich, D., Fetjova, A., Gundelfinger, E.D., Liberman, M.C., Harke, B., et al. (2010). Bassoon and the synaptic ribbon organize $\mathrm{Ca2}+$ channels and vesicles to add release sites and promote refilling. Neuron 68, 724-738.

Fuchs, P.A., Glowatzki, E., and Moser, T. (2003). The afferent synapse of cochlear hair cells. Curr. Opin. Neurobiol. 13, 452-458.

Gebhart, M., Juhasz-Vedres, G., Zuccotti, A., Brandt, N., Engel, J., Trockenbacher, A., Kaur, G., Obermair, G.J., Knipper, M., Koschak, A., et al. (2010). Modulation of Cav1.3 Ca2+ channel gating by Rab3 interacting molecule. Mol. Cell. Neurosci. 44, 246-259. 
Geisler, C.D., Deng, L., and Greenberg, S.R. (1985). Thresholds for primary auditory fibers using statistically defined criteria. J. Acoust. Soc. Am. 77, 1102-1109.

Von Gersdorff, H. (2001). Synaptic ribbons: Versatile signal transducers. Neuron 29, 7-10.

Girotto, G., Vuckovic, D., Buniello, A., Lorente-Cánovas, B., Lewis, M., Gasparini, P., and Steel, K.P. (2014). Expression and replication studies to identify new candidate genes involved in normal hearing function. PLoS One 9, e85352.

Glowatzki, E., and Fuchs, P.A. (2002). Transmitter release at the hair cell ribbon synapse. Nat. Neurosci. 5, 147-154.

Gong, S., Doughty, M., Harbaugh, C.R., Cummins, A., Hatten, M.E., Heintz, N., and Gerfen, C.R. (2007). Targeting Cre Recombinase to Specific Neuron Populations with Bacterial Artificial Chromosome Constructs. J. Neurosci. 27.

Gong, Y., Huang, C., Li, J.Z., Grewe, B.F., Zhang, Y., Eismann, S., and Schnitzer, M.J. (2015). High-speed recording of neural spikes in awake mice and flies with a fluorescent voltage sensor. Science (80-. ). 350, 1361-1366.

Goswami, S.P., Bucurenciu, I., and Jonas, P. (2012). Miniature IPSCs in Hippocampal Granule Cells Are Triggered by Voltage-Gated Ca2+ Channels via Microdomain Coupling. J. Neurosci. 32, 14294-14304.

Goutman, J.D., and Glowatzki, E. (2007). Time course and calcium dependence of transmitter release at a single ribbon synapse. Proc. Natl. Acad. Sci. U. S. A. 104, 16341-16346.

Grabner, C.P., Gandini, M.A., Rehak, R., Le, Y., Zamponi, G.W., and Schmitz, F. (2015). RIM1/2-Mediated Facilitation of Cav1.4 Channel Opening Is Required for Ca2+-Stimulated Release in Mouse Rod Photoreceptors. J. Neurosci. 35, 1313313147.

Grant, L., Yi, E., and Glowatzki, E. (2010). Two Modes of Release Shape the Postsynaptic Response at the Inner Hair Cell Ribbon Synapse. J. Neurosci. 30, 4210-4220.

Grauel, M.K., Maglione, M., Reddy-Alla, S., Willmes, C.G., Brockmann, M.M., Trimbuch, T., Rosenmund, T., Pangalos, M., Vardar, G., Stumpf, A., et al. (2016). RIM-binding protein 2 regulates release probability by fine-tuning calcium channel localization at murine hippocampal synapses. Proc. Natl. Acad. Sci. 113, 1161511620.

Graydon, C.W., Zhang, J., Oesch, N.W., Sousa, A.A., Leapman, R.D., and Diamond, J.S. (2014). Passive Diffusion as a Mechanism Underlying Ribbon Synapse Vesicle Release and Resupply. J. Neurosci. 34, 8948-8962.

Groff, J.A., and Liberman, M.C. (2003). Modulation of cochlear afferent response by the lateral olivocochlear system: activation via electrical stimulation of the inferior colliculus. J. Neurophysiol. 90, 3178-3200.

Guillet, M., Sendin, G., Bourien, J., Puel, J.-L., and Nouvian, R. (2016). Actin Filaments Regulate Exocytosis at the Hair Cell Ribbon Synapse. J. Neurosci. 36, 649-654.

Gundelfinger, E.D., and Fejtova, A. (2012). Molecular organization and plasticity of the cytomatrix at the active zone. Curr. Opin. Neurobiol. 22, 423-430.

Han, Y., Kaeser, P.S., Südhof, T.C., and Schneggenburger, R. (2011). RIM determines Ca2+ channel density and vesicle docking at the presynaptic active zone. Neuron 69, 304-316.

Harlow, M.L., Ress, D., Stoschek, A., Marshall, R.M., and McMahan, U.J. (2001). The architecture of active zone material at the frog's neuromuscular junction. Nature 
409, 479-484.

Harris, D.M., and Dallos, P. (1979). Forward masking of auditory nerve fiber responses. J. Neurophysiol. 42, 1083-1107.

Hernandez, V.H., Gehrt, A., Jing, Z., Hoch, G., Jeschke, M., Strenzke, N., and Moser, T. (2014). Optogenetic Stimulation of the Auditory Nerve. J. Vis. Exp. e52069.

Hibino, H., Pironkova, R., Onwumere, O., Vologodskaia, M., Hudspeth, A.J., and Lesage, F. (2002). RIM binding proteins (RBPs) couple Rab3-interacting molecules (RIMs) to voltage-gated Ca2+ channels. Neuron 34, 411-423.

Hochbaum, D.R., Zhao, Y., Farhi, S.L., Klapoetke, N., Werley, C.A., Kapoor, V., Zou, P., Kralj, J.M., Maclaurin, D., Smedemark-Margulies, N., et al. (2014). All-optical electrophysiology in mammalian neurons using engineered microbial rhodopsins. Nat. Methods 11, 825-833.

Hosoi, N., Sakaba, T., and Neher, E. (2007). Quantitative analysis of calciumdependent vesicle recruitment and its functional role at the calyx of Held synapse. J. Neurosci. 27, 14286-14298.

Hossain, W.A., Antic, S.D., Yang, Y., Rasband, M.N., and Morest, D.K. (2005). Where is the spike generator of the cochlear nerve? Voltage-gated sodium channels in the mouse cochlea. J. Neurosci. 25, 6857-6868.

Howard, J., and Hudspeth, A.J. (1988). Compliance of the hair bundle associated with gating of mechanoelectrical transduction channels in the Bullfrog's saccular hair cell. Neuron 1, 189-199.

Hudspeth, A.J. (2008). Making an Effort to Listen: Mechanical Amplification in the Ear. Neuron 59, 530-545.

Jarsky, T., Tian, M., and Singer, J.H. (2010). Nanodomain control of exocytosis is responsible for the signaling capability of a retinal ribbon synapse. J. Neurosci. 30, 11885-11895.

Jin, L., Han, Z., Platisa, J., Wooltorton, J.R.A., Cohen, L.B., and Pieribone, V.A. (2012). Single Action Potentials and Subthreshold Electrical Events Imaged in Neurons with a Fluorescent Protein Voltage Probe. Neuron 75, 779-785.

Jing, Z., Rutherford, M.A., Takago, H., Frank, T., Fejtova, A., Khimich, D., Moser, T., and Strenzke, N. (2013). Disruption of the Presynaptic Cytomatrix Protein Bassoon Degrades Ribbon Anchorage, Multiquantal Release, and Sound Encoding at the Hair Cell Afferent Synapse. J. Neurosci. 33, 4456-4467.

Johnson, S.L., Olt, J., Cho, S., von Gersdorff, H., and Marcotti, W. (2017). The Coupling between Ca2+ Channels and the Exocytotic Ca2+ Sensor at Hair Cell Ribbon Synapses Varies Tonotopically along the Mature Cochlea. J. Neurosci. 37, 2471-2484.

Joiner, M., and Lee, A. (2015). Voltage-Gated Cav1 Channels in Disorders of Vision and Hearing. Curr. Mol. Pharmacol. 8, 143-148.

Jung, S., Oshima-Takago, T., Chakrabarti, R., Wong, A.B., Jing, Z., Yamanbaeva, G., Picher, M.M., Wojcik, S.M., Gottfert, F., Predoehl, F., et al. (2015a). Rab3interacting molecules 2alpha and 2beta promote the abundance of voltage-gated CaV1.3 Ca2+ channels at hair cell active zones. Proc. Natl. Acad. Sci. U. S. A. 112, E3141-9.

Jung, S., Maritzen, T., Wichmann, C., Jing, Z., Neef, A., Revelo, N.H., Al-Moyed, H., Meese, S., Wojcik, S.M., Panou, I., et al. (2015b). Disruption of adaptor protein 2 (AP-2) in cochlear hair cells impairs vesicle reloading of synaptic release sites and hearing. EMBO J. 34, 2686-2702. 
Kaeser, P.S., Deng, L., Wang, Y., Dulubova, I., Liu, X., Rizo, J., and Südhof, T.C. (2011). RIM proteins tether $\mathrm{Ca} 2+$ channels to presynaptic active zones via a direct PDZ-domain interaction. Cell 144, 282-295.

Kawase, T., and Liberman, M.C. (1992). Spatial organization of the auditory nerve according to spontaneous discharge rate. J. Comp. Neurol. 319, 312-318.

Keen, E.C., and Hudspeth, A.J. (2006). Transfer characteristics of the hair cell's afferent synapse. Proc. Natl. Acad. Sci. U. S. A. 103, 5537-5542.

Khimich, D., Nouvian, R., Pujol, R., Tom Dieck, S., Egner, A., Gundelfinger, E.D., and Moser, T. (2005). Hair cell synaptic ribbons are essential for synchronous auditory signalling. Nature 434, 889-894.

Kiang, N.Y.-S., Pfeiffer, R.R., Warr, W.B., and Backus, A.S.N. (1965). Simulus coding in the cochlear nucleus. Ann. Otol. Rhinol. Laryngol. 41, 463-485.

Kintscher, M., Wozny, C., Johenning, F.W., Schmitz, D., and Breustedt, J. (2013). Role of RIM1 $\alpha$ in short- and long-term synaptic plasticity at cerebellar parallel fibres. Nat. Commun. 4.

Kittel, R.J. (2006). Bruchpilot Promotes Active Zone Assembly, Ca2+ Channel Clustering, and Vesicle Release. Science (80-. ). 312, 1051-1054.

Kiyonaka, S., Wakamori, M., Miki, T., Uriu, Y., Nonaka, M., Bito, H., Beedle, A.M., Mori, E., Hara, Y., De Waard, M., et al. (2007). RIM1 confers sustained activity and neurotransmitter vesicle anchoring to presynaptic Ca2+ channels. Nat. Neurosci. 10, 691-701.

Koschak, A., Reimer, D., Huber, I., Grabner, M., Glossmann, H., Engel, J., and Striessnig, J. (2001). a1D (Cav1.3) Subunits Can Form L-type Ca2+ Channels Activating at Negative Voltages. J. Biol. Chem. 276, 22100-22106.

Koschak, A., Reimer, D., Walter, D., Hoda, J.-C., Heinzle, T., Grabner, M., and Striessnig, J. (2003). Cav1.4alpha1 subunits can form slowly inactivating dihydropyridine-sensitive L-type $\mathrm{Ca} 2+$ channels lacking $\mathrm{Ca} 2+-$ dependent inactivation. J. Neurosci. 23, 6041-6049.

Kralj, J.M., Douglass, A.D., Hochbaum, D.R., Maclaurin, D., and Cohen, A.E. (2011). Optical recording of action potentials in mammalian neurons using a microbial rhodopsin. Nat. Methods 9, 90-95.

Krinner, S., Butola, T., Jung, S., Wichmann, C., and Moser, T. (2017). RIM-binding protein 2 promotes a large number of $\mathrm{CaV} 1.3 \mathrm{Ca2}+$-channels and contributes to fast synaptic vesicle replenishment at hair cell active zones. Front. Cell. Neurosci. (accepted for publication).

Kulkarni, R.U., and Miller, E.W. (2017). Voltage Imaging: Pitfalls and Potential. Biochemistry acs.biochem.7b00490.

Lacas-Gervais, S., Guo, J., Strenzke, N., Scarfone, E., Kolpe, M., Jahkel, M., De Camilli, P., Moser, T., Rasband, M.N., and Solimena, M. (2004). BetalVSigma1 spectrin stabilizes the nodes of Ranvier and axon initial segments. J. Cell Biol. 166, 983-990.

Lee, J.S., Ho, W.-K., Neher, E., and Lee, S.-H. (2013). Superpriming of synaptic vesicles after their recruitment to the readily releasable pool. Proc. Natl. Acad. Sci. U. S. A. 110, 15079-15084.

Lenzi, D., and Von Gersdorff, H. (2001). Structure suggests function: The case for synaptic ribbons as exocytotic nanomachines. BioEssays 23, 831-840.

Lenzi, D., Runyeon, J.W., Crum, J., Ellisman, M.H., and Roberts, W.M. (1999). Synaptic vesicle populations in saccular hair cells reconstructed by electron 
tomography. J. Neurosci. 19, 119-132.

Lenzi, D., Crum, J., Ellisman, M.H., and Roberts, W.M. (2002). Depolarization redistributes synaptic membrane and creates a gradient of vesicles on the synaptic body at a ribbon synapse. Neuron 36, 649-659.

Li, G.-L., Keen, E., Andor-Ardo, D., Hudspeth, A.J., and von Gersdorff, H. (2009). The Unitary Event Underlying Multiquantal EPSCs at a Hair Cell's Ribbon Synapse. J. Neurosci. 29, 7558-7568.

Liberman, M.C. (1978). Auditory-nerve response from cats raised in a low-noise chamber. J. Acoust. Soc. Am. 63, 442-455.

Liberman, M.C. (1980). Efferent synapses in the inner hair cell area of the cat cochlea: An electron microscopic study of serial sections. Hear. Res. 3, 189-204.

Liberman, M.C. (1982). The cochlear frequency map for the cat: Labeling auditorynerve fibers of known characteristic frequency. J. Acoust. Soc. Am. 72, 1441.

Liberman, M.C., and Oliver, M.E. (1984). Morphometry of intracellularly labeled neurons of the auditory nerve: Correlations with functional properties. J. Comp. Neurol. 223, 163-176.

Liberman, L.D., Wang, H., and Liberman, M.C. (2011). Opposing Gradients of Ribbon Size and AMPA Receptor Expression Underlie Sensitivity Differences among Cochlear-Nerve/Hair-Cell Synapses. J. Neurosci. 31, 801-808.

Limbach, C., Laue, M.M., Wang, X., Hu, B., Thiede, N., Hultqvist, G., and Kilimann, M.W. (2011). Molecular in situ topology of Aczonin/Piccolo and associated proteins at the mammalian neurotransmitter release site. Proc. Natl. Acad. Sci. 108, E392E401.

Lindau, M., and Neher, E. (1988). Patch-clamp techniques for time-resolved capacitance measurements in single cells. Pflugers Arch. 411, 137-146.

Liu, K.S.Y., Siebert, M., Mertel, S., Knoche, E., Wegener, S., Wichmann, C., Matkovic, T., Muhammad, K., Depner, H., Mettke, C., et al. (2011). RIM-Binding Protein, a Central Part of the Active Zone, Is Essential for Neurotransmitter Release. Science (80-. ). 334, 1565-1569.

Lou, S., Adam, Y., Weinstein, E.N., Williams, E., Williams, K., Parot, V., Kavokine, N., Liberles, S., Madisen, L., Zeng, H., et al. (2016). Genetically Targeted All-Optical Electrophysiology with a Transgenic Cre-Dependent Optopatch Mouse. J. Neurosci. 36, 11059-11073.

Luebke, J.I., Dunlap, K., and Turner, T.J. (1993). Multiple calcium channel types control glutamatergic synaptic transmission in the hippocampus. Neuron 11, 895902.

Luo, F., Liu, X., Südhof, T.C., and Acuna, C. (2017). Efficient stimulus-secretion coupling at ribbon synapses requires RIM-binding protein tethering of L-type $\mathrm{Ca}^{2+}$ channels. Proc. Natl. Acad. Sci. 201702991.

Maclaurin, D., Venkatachalam, V., Lee, H., and Cohen, A.E. (2013). Mechanism of voltage-sensitive fluorescence in a microbial rhodopsin. Proc. Natl. Acad. Sci. U. S. A. $110,5939-5944$.

Madisen, L., Garner, A.R., Shimaoka, D., Chuong, A.S., Klapoetke, N.C., Li, L., van der Bourg, A., Niino, Y., Egolf, L., Monetti, C., et al. (2015). Transgenic mice for intersectional targeting of neural sensors and effectors with high specificity and performance. Neuron 85, 942-958.

Mansergh, F., Orton, N.C., Vessey, J.P., Lalonde, M.R., Stell, W.K., Tremblay, F., Barnes, S., Rancourt, D.E., and Bech-Hansen, N.T. (2005). Mutation of the calcium 
channel gene Cacna1f disrupts calcium signaling, synaptic transmission and cellular organization in mouse retina. Hum. Mol. Genet. 14, 3035-3046.

Matthews, G., and Sterling, P. (2008). Evidence That Vesicles Undergo Compound Fusion on the Synaptic Ribbon. J. Neurosci. 28, 5403-5411.

Matveev, V., Bertram, R., and Sherman, A. (2011). Calcium cooperativity of exocytosis as a measure of Ca2+ channel domain overlap. Brain Res. 1398, 126-138.

Maxeiner, S., Luo, F., Tan, A., Schmitz, F., Südhof, T.C., Allwardt, B., Lall, A., Broeckerhoff, S., Dowling, J., Alpadi, K., et al. (2016). How to make a synaptic ribbon: RIBEYE deletion abolishes ribbons in retinal synapses and disrupts neurotransmitter release. EMBO J. 35, 1098-1114.

McLean, W.J., Smith, K.A., Glowatzki, E., and Pyott, S.J. (2009). Distribution of the $\mathrm{Na}, \mathrm{K}-\mathrm{ATP}$ ase alpha subunit in the rat spiral ganglion and organ of corti. J. Assoc. Res. Otolaryngol. 10, 37-49.

Melcher, J.R., Guinan, J.J., Knudson, I.M., and Kiang, N.Y. (1996). Generators of the brainstem auditory evoked potential in cat. II. Correlating lesion sites with waveform changes. Hear. Res. 93, 28-51.

Merchan-Perez, A., and Liberman, M.C. (1996). Ultrastructural differences among afferent synapses on cochlear hair cells: Correlations with spontaneous discharge rate. J. Comp. Neurol. 371, 208-221.

Meyer, A.C., and Moser, T. (2010). Structure and function of cochlear afferent innervation. Curr. Opin. Otolaryngol. Head Neck Surg. 18, 441-446.

Meyer, A.C., Frank, T., Khimich, D., Hoch, G., Riedel, D., Chapochnikov, N.M., Yarin, Y.M., Harke, B., Hell, S.W., Egner, A., et al. (2009). Tuning of synapse number, structure and function in the cochlea. Nat. Neurosci. 12, 444-453.

Miesenböck, G., De Angelis, D.A., and Rothman, J.E. (1998). Visualizing secretion and synaptic transmission with $\mathrm{pH}$-sensitive green fluorescent proteins. Nature 394, 192-195.

Mittelstaedt, T., and Schoch, S. (2007). Structure and evolution of RIM-BP genes: Identification of a novel family member. Gene 403, 70-79.

Morgans, C.W. (2001). Localization of the alpha(1F) calcium channel subunit in the rat retina. Invest. Ophthalmol. Vis. Sci. 42, 2414-2418.

Moser, T., and Beutner, D. (2000). Kinetics of exocytosis and endocytosis at the cochlear inner hair cell afferent synapse of the mouse. Proc Natl Acad Sci U S A 97, 883-888.

Moser, T., and Starr, A. (2016). Auditory neuropathy - neural and synaptic mechanisms. Nat. Rev. Neurol. 12, 135-149.

Moser, T., and Vogl, C. (2016). New insights into cochlear sound encoding. F1000Research 5, 2081.

Moser, T., Neef, A., and Khimich, D. (2006a). Mechanisms underlying the temporal precision of sound coding at the inner hair cell ribbon synapse. J. Physiol. 576, 5562.

Moser, T., Strenzke, N., Meyer, a, Lesinski-Schiedat, a, Lenarz, T., Beutner, D., Foerst, a, Lang-Roth, R., von Wedel, H., Walger, M., et al. (2006b). [Diagnosis and therapy of auditory synaptopathy/neuropathy]. HNO 54, 833-839.

Müller, U. (2008). Cadherins and mechanotransduction by hair cells. Curr. Opin. Cell Biol. 20, 557-566.

Müller, M., Genç, Ö., and Davis, G.W. (2015). RIM-binding protein links synaptic 
homeostasis to the stabilization and replenishment of high release probability vesicles. Neuron 85, 1056-1069.

Nagai, T., Ibata, K., Park, E.S., Kubota, M., Mikoshiba, K., and Miyawaki, A. (2002). A variant of yellow fluorescent protein with fast and efficient maturation for cellbiological applications. Nat. Biotechnol. 20, 87-90.

Neef, J., Jung, S., Wong, A.B., Reuter, K., Pangršič, T., Chakrabarti, R., Kügler, S., Lenz, C., Nouvian, R., Boumil, R.M., et al. (2014). Modes and Regulation of Endocytic Membrane Retrieval in Mouse Auditory Hair Cells. J. Neurosci. 34, 705716.

Neher, E. (1998). Vesicle pools and Ca2+ microdomains: New tools for understanding their roles in neurotransmitter release. Neuron 20, 389-399.

Neher, E., and Sakaba, T. (2008). Multiple Roles of Calcium lons in the Regulation of Neurotransmitter Release. Neuron 59, 861-872.

Nouvian, R., Beutner, D., Parsons, T.D., and Moser, T. (2006). Structure and function of the hair cell ribbon synapse. J. Membr. Biol. 209, 153-165.

Nouvian, R., Neef, J., Bulankina, A. V, Reisinger, E., Pangršič, T., Frank, T., Sikorra, S., Brose, N., Binz, T., and Moser, T. (2011). Exocytosis at the hair cell ribbon synapse apparently operates without neuronal SNARE proteins. Nat. Neurosci. 14, 411-413.

Ohlemiller, K.K., and Echteler, S.M. (1990). Functional correlates of characteristic frequency in single cochlear nerve fibers of the Mongolian gerbil. J. Comp. Physiol. A. 167, 329-338.

Ohn, T.-L., Rutherford, M.A., Jing, Z., Jung, S., Duque-Afonso, C.J., Hoch, G., Picher, M.M., Scharinger, A., Strenzke, N., and Moser, T. (2016). Hair cells use active zones with different voltage dependence of $\mathrm{Ca} 2+$ influx to decompose sounds into complementary neural codes. Proc. Natl. Acad. Sci. U. S. A. 113, E4716-25.

Ohtsuka, T., Takao-Rikitsu, E., Inoue, E., Inoue, M., Takeuchi, M., Matsubara, K., Deguchi-Tawarada, M., Satoh, K., Morimoto, K., Nakanishi, H., et al. (2002). CAST: A novel protein of the cytomatrix at the active zone of synapses that forms a ternary complex with RIM1 and Munc13-1. J. Cell Biol. 158, 577-590.

Pangršič, T., Lasarow, L., Reuter, K., Takago, H., Schwander, M., Riedel, D., Frank, T., Tarantino, L.M., Bailey, J.S., Strenzke, N., et al. (2010). Hearing requires otoferlin-dependent efficient replenishment of synaptic vesicles in hair cells. Nat. Neurosci. 13, 869-876.

Pangršič, T., Reisinger, E., and Moser, T. (2012). Otoferlin: a multi-C2 domain protein essential for hearing. Trends Neurosci. 35, 671-680.

Pangršič, T., Gabrielaitis, M., Michanski, S., Schwaller, B., Wolf, F., Strenzke, N., and Moser, T. (2015). EF-hand protein $\mathrm{Ca} 2+$ buffers regulate $\mathrm{Ca} 2+$ influx and exocytosis in sensory hair cells. Proc. Natl. Acad. Sci. U. S. A. 112, E1028-37.

Parsons, T.D., and Sterling, P. (2003). Synaptic ribbon: Conveyor belt or safety belt? Neuron 37, 379-382.

Parsons, T.D., Lenzi, D., Almers, W., and Roberts, W.M. (1994). Calcium-triggered exocytosis and endocytosis in an isolated presynaptic cell: Capacitance measurements in saccular hair cells. Neuron 13, 875-883.

Phillips, G.R., Huang, J.K., Wang, Y., Tanaka, H., Shapiro, L., Zhang, W., Shan, W.S., Arndt, K., Frank, M., Gordon, R.E., et al. (2001). The presynaptic particle web: Ultrastructure, composition, dissolution, and reconstitution. Neuron 32, 63-77.

Piao, H.H., Rajakumar, D., Kang, B.E., Kim, E.H., and Baker, B.J. (2015). 
Combinatorial mutagenesis of the voltage-sensing domain enables the optical resolution of action potentials firing at $60 \mathrm{~Hz}$ by a genetically encoded fluorescent sensor of membrane potential. J. Neurosci. 35, 372-385.

Picher, M.M., Oprişoreanu, A.-M., Jung, S., Michel, K., Schoch, S., and Moser, T. (2017). Rab Interacting Molecules 2 and 3 Directly Interact with the Pore-Forming CaV1.3 Ca2+ Channel Subunit and Promote Its Membrane Expression. Front. Cell. Neurosci. 11.

Pickles, J.O., Comis, S.D., and Osborne, M.P. (1984). Cross-links between stereocilia in the guinea pig organ of Corti, and their possible relation to sensory transduction. Hear. Res. 15, 103-112.

Platzer, J., Engel, J., Schrott-Fischer, A., Stephan, K., Bova, S., Chen, H., Zheng, H., and Striessnig, J. (2000). Congenital Deafness and Sinoatrial Node Dysfunction in Mice Lacking Class D L-Type Ca2+ Channels. Cell 102, 89-97.

Le Prell, C.G., Halsey, Kä., Hughes, L.F., Dolan, D.F., and Bledsoe, S.C. (2005). Disruption of Lateral Olivocochlear Neurons via a Dopaminergic Neurotoxin Depresses Sound-Evoked Auditory Nerve Activity. J. Assoc. Res. Otolaryngol. 6, 48-62.

Purves, D., Augustine, G.J., Fitzpatrick, D., Hall, W.C., Lamantia, A.-S., Mcnamara, J.O., and Willians, S.M. (2004). Neuroscience.

Reichenbach, T., and Hudspeth, A.J. (2010). A ratchet mechanism for amplification in low-frequency mammalian hearing. Proc. Natl. Acad. Sci. 107, 4973-4978.

Reijntjes, D.O.J., and Pyott, S.J. (2016). The afferent signaling complex: Regulation of type I spiral ganglion neuron responses in the auditory periphery. Hear. Res. 336, $1-16$.

Ricci, A.J., Kennedy, H.J., Crawford, A.C., and Fettiplace, R. (2005). The transduction channel filter in auditory hair cells. J. Neurosci. 25, 7831-7839.

Rio, M.S., and Pareschi, G. (2001). Global optimization and relectivity data fitting for X-ray multilayer mirrors by means of genetic algorithms. 4145, 88-96.

Rizzoli, S.O., and Betz, W.J. (2005). Synaptic vesicle pools. Nat. Rev. Neurosci. 6, 5769.

Ross, S.E., Mardinly, A.R., McCord, A.E., Zurawski, J., Cohen, S., Jung, C., Hu, L., Mok, S.I., Shah, A., Savner, E.M., et al. (2010). Loss of inhibitory interneurons in the dorsal spinal cord and elevated itch in Bhlhb5 mutant mice. Neuron 65, 886898.

Ruel, J., Nouvian, R., D’Aldin, C.G., Pujol, R., Eybalin, M., and Puel, J.L. (2001). Dopamine inhibition of auditory nerve activity in the adult mammalian cochlea. Eur. J. Neurosci. 14, 977-986.

Rutherford, M.A., and Pangršič, T. (2012). Molecular anatomy and physiology of exocytosis in sensory hair cells. Cell Calcium 52, 327-337.

Rutherford, M.A., and Roberts, W.M. (2006). Frequency selectivity of synaptic exocytosis in frog saccular hair cells. Proc. Natl. Acad. Sci. U. S. A. 103, 2898 2903.

Rutherford, M.A., Chapochnikov, N.M., and Moser, T. (2012). Spike Encoding of Neurotransmitter Release Timing by Spiral Ganglion Neurons of the Cochlea. J. Neurosci. 32, 4773-4789.

Sachs, M.B., and Abbas, P.J. (1974). Rate versus level functions for auditory-nerve fibers in cats: tone-burst stimuli. J. Acoust. Soc. Am. 56, 1835-1847. 
Safieddine, S., and Wenthold, R.J. (1999). SNARE complex at the ribbon synapses of cochlear hair cells: Analysis of synaptic vesicle- and synaptic membrane-associated proteins. Eur. J. Neurosci. 11, 803-812.

Safieddine, S., El-Amraoui, A., and Petit, C. (2012). The auditory hair cell ribbon synapse: from assembly to function. Annu. Rev. Neurosci. 35, 509-528.

Sankaranarayanan, S., De Angelis, D., Rothman, J.E., and Ryan, T.A. (2000). The use of pHluorins for optical measurements of presynaptic activity. Biophys. J. 79, 21992208.

Schmitz, F., Königstorfer, A., and Südhof, T.C. (2000). RIBEYE, a component of synaptic ribbons: A protein's journey through evolution provides insight into synaptic ribbon function. Neuron 28, 857-872.

Schnee, M.E., Lawton, D.M., Furness, D.N., Benke, T.A., and Ricci, A.J. (2005). Auditory hair cell-afferent fiber synapses are specialized to operate at their best frequencies. Neuron 47, 243-254.

Schnee, M.E., Santos-Sacchi, J., Castellano-Muñoz, M., Kong, J.-H., and Ricci, A.J. (2011). Calcium-dependent synaptic vesicle trafficking underlies indefatigable release at the hair cell afferent fiber synapse. Neuron 70, 326-338.

Schneggenburger, R., and Neher, E. (2005). Presynaptic calcium and control of vesicle fusion. Curr. Opin. Neurobiol. 15, 266-274.

Schneider, C.A., Rasband, W.S., and Eliceiri, K.W. (2012). NIH Image to ImageJ: 25 years of image analysis. Nat. Methods 9, 671-675.

Schoch, S., and Gundelfinger, E.D. (2006). Molecular organization of the presynaptic active zone. Cell Tissue Res. 326, 379-391.

Scholz, K.P., and Miller, R.J. (1995). Developmental changes in presynaptic calcium channels coupled to glutamate release in cultured rat hippocampal neurons. J. Neurosci. 15, 4612-4617.

Seal, R.P., Akil, O., Yi, E., Weber, C.M., Grant, L., Yoo, J., Clause, A., Kandler, K., Noebels, J.L., Glowatzki, E., et al. (2008). Sensorineural deafness and seizures in mice lacking vesicular glutamate transporter 3 . Neuron 57, 263-275.

Siemens, J., Lillo, C., Dumont, R.A., Reynolds, A., Williams, D.S., Gillespie, P.G., and Müller, U. (2004). Cadherin 23 is a component of the tip link in hair-cell stereocilia. Nature 428, 950-955.

Sobkowicz, H.M., Rose, J.E., Scott, G.E., and Slapnick, S.M. (1982). Ribbon synapses in the developing intact and cultured organ of Corti in the mouse. J. Neurosci. 2, 942-957.

Söllner, C., Rauch, G.-J., Siemens, J., Geisler, R., Schuster, S.C., Müller, U., and Nicolson, T. (2004). Mutations in cadherin 23 affect tip links in zebrafish sensory hair cells. Nature 428, 955-959.

Spassova, M.A., Avissar, M., Furman, A.C., Crumling, M.A., Saunders, J.C., and Parsons, T.D. (2004). Evidence that rapid vesicle replenishment of the synaptic ribbon mediates recovery from short-term adaptation at the hair cell afferent synapse. JARO - J. Assoc. Res. Otolaryngol. 5, 376-390.

Sterling, P., and Matthews, G. (2005). Structure and function of ribbon synapses. Trends Neurosci. 28, 20-29.

Strom, T.M., Nyakatura, G., Apfelstedt-Sylla, E., Hellebrand, H., Lorenz, B., Weber, B.H.F., Wutz, K., Gutwillinger, N., Ruther, K., Drescher, B., et al. (1998). An L-type calcium-channel gene mutated in incomplete $X$-linked congenital stationary night blindness. Nat Genet 19, 260-263. 
Südhof, T.C. (2012). The presynaptic active zone. Neuron 75, 11-25.

Szule, J.A., Harlow, M.L., Jung, J.H., De-Miguel, F.F., Marshall, R.M., and McMahan, U.J. (2012). Regulation of synaptic vesicle docking by different classes of macromolecules in active zone material. PLoS One 7.

Taberner, A., and Liberman, M. (2005). Response properties of single auditory nerve fibers in the mouse. J. Neurophysiol. 557-569.

Takahashi, T., and Momiyama, A. (1993). Different types of calcium channels mediate central synaptic transmission. Nature 366, 156-158.

Thoreson, W.B., Rabl, K., Townes-Anderson, E., and Heidelberger, R. (2004). A highly $\mathrm{Ca2+-sensitive} \mathrm{pool} \mathrm{of} \mathrm{vesicles} \mathrm{contributes} \mathrm{to} \mathrm{linearity} \mathrm{at} \mathrm{the} \mathrm{rod} \mathrm{photoreceptor}$ ribbon synapse. Neuron 42, 595-605.

Tom Dieck, S., Altrock, W.D., Kessels, M.M., Qualmann, B., Regus, H., Brauner, D., Fejtová, A., Bracko, O., Gundelfinger, E.D., and Brandstätter, J.H. (2005). Molecular dissection of the photoreceptor ribbon synapse: Physical interaction of Bassoon and RIBEYE is essential for the assembly of the ribbon complex. J. Cell Biol. 168, 825-836.

Vaithianathan, T., Henry, D., Akmentin, W., and Matthews, G. (2016). Nanoscale dynamics of synaptic vesicle trafficking and fusion at the presynaptic active zone. Elife 5.

Vogl, C., Cooper, B.H., Neef, J., Wojcik, S.M., Reim, K., Reisinger, E., Brose, N., Rhee, J.-S., Moser, T., and Wichmann, C. (2015). Unconventional molecular regulation of synaptic vesicle replenishment in cochlear inner hair cells. J. Cell Sci. 128, 638644.

Wagh, D.A., Rasse, T.M., Asan, E., Hofbauer, A., Schwenkert, I., Dürrbeck, H., Buchner, S., Dabauvalle, M.C., Schmidt, M., Qin, G., et al. (2006). Bruchpilot, a protein with homology to ELKS/CAST, is required for structural integrity and function of synaptic active zones in Drosophila. Neuron 49, 833-844.

Wang, L.-Y., and Kaczmarek, L.K. (1998). High-frequency firing helps replenish the readily releasable pool of synaptic vesicles. Nature 394, 384-388.

Wang, S.S.H., Held, R.G., Wong, M.Y., Liu, C., Karakhanyan, A., and Kaeser, P.S. (2016). Fusion Competent Synaptic Vesicles Persist upon Active Zone Disruption and Loss of Vesicle Docking. Neuron 91, 777-791.

Wang, Y., Sugita, S., and Südhof, T.C. (2000). The RIM/NIM Family of Neuronal C2 Domain Proteins. J. Biol. Chem. 275, 20033-20044.

Wang, Y., Liu, X., Biederer, T., and Südhof, T.C. (2002). A family of RIM-binding proteins regulated by alternative splicing: Implications for the genesis of synaptic active zones. Proc. Natl. Acad. Sci. U. S. A. 99, 14464-14469.

Weimer, R.M., Gracheva, E.O., Meyrignac, O., Miller, K.G., Richmond, J.E., and Bessereau, J.-L. (2006). UNC-13 and UNC-10/Rim Localize Synaptic Vesicles to Specific Membrane Domains. J. Neurosci. 26, 8040-8047.

Wheeler, D.B., Randall, A., and Tsien, R.W. (1994). Roles of N-type and Q-type Ca2+ channels in supporting hippocampal synaptic transmission. Science 264, 107-111.

Wichmann, C., and Moser, T. (2015). Relating structure and function of inner hair cell ribbon synapses. Cell Tissue Res. 361, 95-114.

Wichmann, C., and Sigrist, S.J. (2010). The Active Zone T-Bar-A Plasticity Module? J. Neurogenet. 24, 133-145.

Wittig, J.H., and Parsons, T.D. (2008). Synaptic ribbon enables temporal precision of 
hair cell afferent synapse by increasing the number of readily releasable vesicles: a modeling study. J. Neurophysiol. 100, 1724-1739.

Wong, A.B., Rutherford, M.A., Gabrielaitis, M., Pangršič, T., Göttfert, F., Frank, T., Michanski, S., Hell, S., Wolf, F., Wichmann, C., et al. (2014). Developmental refinement of hair cell synapses tightens the coupling of $\mathrm{Ca} 2+$ influx to exocytosis. EMBO J. 33, 247-264.

Wu, J.S., Young, E.D., and Glowatzki, E. (2016). Maturation of Spontaneous Firing Properties after Hearing Onset in Rat Auditory Nerve Fibers: Spontaneous Rates, Refractoriness, and Interfiber Correlations. J. Neurosci. 36, 10584-10597.

Wu, L.G., Westenbroek, R.E., Borst, J.G., Catterall, W.A., and Sakmann, B. (1999). Calcium channel types with distinct presynaptic localization couple differentially to transmitter release in single calyx-type synapses. J. Neurosci. 19, 726-736.

Xu, W., and Lipscombe, D. (2001). Neuronal Ca(V)1.3alpha(1) L-type channels activate at relatively hyperpolarized membrane potentials and are incompletely inhibited by dihydropyridines. J. Neurosci. 21, 5944-5951.

Xu, Y., Zou, P., and Cohen, A.E. (2017). Voltage imaging with genetically encoded indicators. Curr. Opin. Chem. Biol. 39, 1-10.

Yan, D., Ouyang, X.M., Zhu, X., Du, L.L., Chen, Z.Y., and Liu, X.Z. (2005). Refinement of the DFNA41 locus and candidate genes analysis. J. Hum. Genet. 50, 516-522.

Yates, G.K., Winter, I.M., and Robertson, D. (1990). Basilar membrane nonlinearity determines auditory nerve rate-intensity functions and cochlear dynamic range. Hear. Res. 45, 203-219.

Zampini, V., Johnson, S.L., Franz, C., Lawrence, N.D., Münkner, S., Engel, J., Knipper, M., Magistretti, J., Masetto, S., and Marcotti, W. (2010). Elementary properties of $\mathrm{CaV} 1.3 \mathrm{Ca} 2+$ channels expressed in mouse cochlear inner hair cells. J Physiol 588, 187-199.

Zampini, V., Johnson, S.L., Franz, C., Knipper, M., Holley, M.C., Magistretti, J., Masetto, S., and Marcotti, W. (2013). Burst activity and ultrafast activation kinetics of $\mathrm{CaV} 1.3 \mathrm{Ca}^{2+}$ channels support presynaptic activity in adult gerbil hair cell ribbon synapses. J. Physiol. 591, 3811-3820.

Zhai, R.G., and Bellen, H.J. (2004). The Architecture of the Active Zone in the Presynaptic Nerve Terminal. Physiology 19, 262-270.

Zheng, J., Shen, W., He, D.Z., Long, K.B., Madison, L.D., and Dallos, P. (2000). Prestin is the motor protein of cochlear outer hair cells. Nature 405, 149-155. 


\section{List of abbreviations}

2D

3D

AAV

ABR

AMPA

ANF

AZ

$\mathrm{Ca}^{2+}$

$\left[\mathrm{Ca}^{2+}\right]_{\mathrm{e}}$

$\mathrm{CAZ}$

$\mathrm{C}_{\mathrm{m}}$

$\Delta \mathrm{C}_{\mathrm{m}}$

CtBP2

CV

$\mathrm{dB}$ SPL

DPOAE

EDTA

EGTA

EPSC

EPSP

$\mathrm{F}$

$\mathrm{F}_{0}$

$\Delta \mathrm{F}$

$\Delta F / F_{0}$

$\mathrm{FA}$

fF

FWHM

$\mathrm{G}$

GEVI

GSDB

HEPES

$\mathrm{Hz}$

ICa

IV

IHC

$\mathrm{k}$

$\mathrm{K}^{+}$

$\mathrm{kHz}$

LJP

$M$

MP

MET

$\mathrm{mM}$

MP

MRS

$\mathrm{ms}$

$\mathrm{mV}$

$\mathrm{Na}^{+}$

NA

$\mathrm{nm}$
2 dimensional

3 dimensional

Adeno-associated virus

Auditory brainstem response

a-amino-3-hydroxy-5-methyl-4-isoxazolepropionic acid

Auditory nerve fiber

Active zone

Calcium ion

Extracellular $\mathrm{Ca}^{2+}$ concentration

Cytomatrix of the active zone

Membrane capacitance

Membrane capacitance increment

C-terminal binding protein 2

Coefficient of variance

Decibel sound pressure level (IEC definition: $0 \mathrm{~dB} S P L=2 \times 10^{-5} \mathrm{~Pa}$ for gas)

Distortion-product otoacoustic emission

Ethylenediaminetetraacetic acid

Ethylene glycol-bis( $\beta$-amioethyl ether)-N,N,N',N'-tetraacetic acid

Excitatory postsynaptic current

Excitatory postsynaptic potential

Fluorescence

Baseline fluorescence

Fluorescence increment

Normalized change in fluorescence intensity

Formaldehyde

Femtofarad $\left(10^{-15} \mathrm{~F}\right)$

Full width at half maximum

Conductance

Genetically encoded voltage indicator

Goat serum diluted buffer

HEPES-buffered Hanks' balanced salts solution

Hertz $\left(\right.$ second $\left.^{-1}\right)$

$\mathrm{Ca}^{2+}$-current

Current-voltage relationship

Inner hair cell

Slope of activation

Potassium ion

Kilohertz $\left(10^{3} \mathrm{~Hz}\right)$

Liquid junction potential

Molar

Membrane-proximal

Mechano-electrical transducer

Millimolar $\left(10^{-3} \mathrm{M}\right)$

Membrane proximal

Modified Ringer's solution

Millisecond $\left(10^{-3} \mathrm{~s}\right)$

Millivolt $\left(10^{-3} \mathrm{~V}\right)$

Sodium ion

Numerical aperture

Nanometer $\left(10^{-9} \mathrm{~m}\right)$ 


\begin{tabular}{|c|c|}
\hline NMJ & Neuromascular junction \\
\hline $\mathrm{OHC}$ & Outer hair cell \\
\hline $\mathrm{G} \Omega$ & Gigaohm $\left(10^{9} \Omega\right)$ \\
\hline$M \Omega$ & Megaohm $\left(10^{6} \Omega\right)$ \\
\hline p & Postnatal day \\
\hline $\mathrm{pA}$ & Picoampere $\left(10^{-12} \mathrm{~A}\right)$ \\
\hline $\mathrm{pC}$ & Picocoulomb $\left(10^{-12} \mathrm{C}\right)$ \\
\hline PBS & Phosphate-buffered saline \\
\hline PD & Presynaptic density \\
\hline PFA & Paraformaldehyde \\
\hline PSTH & Peri-stimulus time histogram \\
\hline$Q_{\mathrm{Ca}}$ & Charge integral of $\mathrm{Ca}^{2+}$-current \\
\hline RA & Ribbon-associated \\
\hline RIM & Rab3 interacting molecule \\
\hline RIM-BP & Rab3 interacting molecule-binding protein \\
\hline RRP & Readily releasable pool \\
\hline $\mathrm{R}_{\mathrm{S}}$ & Series resistance \\
\hline s & Second \\
\hline SD & Standard deviation \\
\hline SEM & Standard error of the mean \\
\hline SGN & Spiral ganglion neuron \\
\hline SNARE & soluble N-ethylmaleimide-sensitive factor attachment protein receptors \\
\hline SR & Spontaneous spike rate \\
\hline STED & Stimulated emission depletion \\
\hline SV & Synaptic vesicle \\
\hline TAMRA & Carboxytetramethylrhodamine \\
\hline TEA & Tetraethylammonium \\
\hline$V_{m}$ & Membrane potential \\
\hline $\mathrm{V}_{\text {rev }}$ & Reversal potential \\
\hline $\mathrm{W} / \mathrm{cm}^{2}$ & Watt $\left(\right.$ Joule $x$ second $^{-1}$ ) per square centimeter \\
\hline$\mu \mathrm{M}$ & Micromolar $\left(10^{-6} \mathrm{M}\right)$ \\
\hline$\mu \mathrm{m}$ & Micrometer $\left(10^{-6} \mathrm{~m}\right)$ \\
\hline
\end{tabular}




\section{Acknowledgements}

I thank Prof. Tobias Moser for giving me the great opportunity to work in his laboratory. I really appreciate his constant support, his interest, guidance, scientific discussions and I am thankful for giving me the chance to exchange and connect with colleagues within and outside the field. Prof. Tobias Moser was not only a great scientific supervisor, but also a wonderful mentor.

Moreover, I thank Prof. Erwin Neher and Prof. Stefan Hell, the members of my thesis advisory committee for their great support, encouragement, ideas and useful advice.

I am very grateful to all members of the InnerEarLab for providing a stimulating, positive and enjoyable work environment. I would like to especially thank Dr. Kai Bodensiek for his expertise and help building the wide-field voltage imaging setup; Dr. Jakob Neef for excellent technical and scientific advice in many aspects; Dr. Aaron Benson Wong for initially teaching me hair cell patch-clamp recordings and confocal $\mathrm{Ca}^{2+}$-imaging; Dr. SangYong Jung, Tanvi Butola and Prof. Carolin Wichmann for contributing experimental data for the RIM-BP2 project; Rituparna Chakrabarti for help with the electron microscopy analysis; Nadine Dietrich and Stefan Thom for measuring ABRs; Gerhard Hoch for providing his Matlab codes for image analysis; Thomas Gundlach and Daniela Marschall for taking care of the mice and Christiane Senger Freitag and Sandra Gerke for their excellent technical assistance.

Furthermore, I thank Prof. Adam Cohen and Prof. Lisa Goodrich and their lab members for hosting me in the Harvard University and Harvard Medical School in Boston, for providing transgenic mice and virus constructs and ongoing help and scientific input for establishing voltage imaging in the InnerEarLab.

I would also like to thank the Ph.D. program "Molecular Biology" - International Max Planck Research School and the Göttingen Graduate School for Neurosciences, Biophysics, and Molecular Biosciences (GGNB) (DFG grant GSC 226) at the Georg August University Göttingen, especially Dr. Steffen Burkhardt and Kerstin Grüniger and the SFB889 for financial support, great scientific retreats and scientific exchange.

Finally, I deeply thank my parents for their unfailing support, understanding and encouragement, interest in my work, advice and love. 


\section{Declaration}

This thesis has been written independently and with no other sources and aids than quoted.

Stefanie Krinner

Göttingen, October 9 ${ }^{\text {th }}, 2017$ 


\section{Curriculum Vitae}

\section{Personal Details}

Stefanie Krinner

Karolinenweg 9

D-37075 Göttingen, Germany

Phone: +49-551-39-22837

Email: steffi.krinner@t-online.de

Year of Birth: 1989

Nationality: German

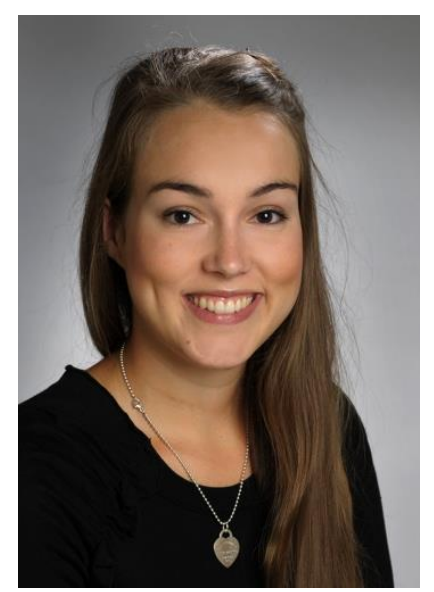

\section{Education}

\section{$2014-2017$ University of Göttingen PhD in Neuroscience}

Supervisor: Prof. Tobias Moser

Thesis committee: Prof. Erwin Neher, Prof. Stefan Hell

Location and Funding: Institute for Auditory Neuroscience \& InnerEarLab, Collaborative Research Center 889, University Medical Center Göttingen, Germany.

Program: "MSc/PhD program Molecular Biology" International Max-Planck Research School (IMPRS), Göttingen Graduate School for Neurosciences, Biophysics and Molecular Biosciences (GGNB)

Collaborative research projects abroad: 02/2016 - 04/2016

Prof. Adam Cohen, Harvard University, Boston USA

Prof. Lisa Goodrich, Harvard Medical School, Boston, USA

\section{2 - 2014 University of Göttingen: M.Sc. in Molecular Biology}

Dissertation project: Spatiotemporally resolved imaging of single active zones

Supervisor: Dr. Aaron Benson Wong

Referees: Prof. Tobias Moser, Prof. Erwin Neher

Location: Institute for Auditory Neuroscience \& InnerEarLab, Collaborative Research Center 889, University Medical Center Göttingen, Germany.

Program/Stipend: "MSc/PhD program Molecular Biology" International Max-Planck Research School (IMPRS), Göttingen Graduate School for Neurosciences, Biophysics and Molecular Biosciences (GGNB) 


\section{9 - 2012 Ludwig Maximilian University of Munich B.Sc. in Biology}

Dissertation project: Characterization of the expression pattern and biochemical interactions of the leucin-rich repeat protein Lingo-1

Supervisor: Dr. Irina Dudanova; Prof. Rüdiger Klein

Location: Department of Molecules - Signaling - Development, Max-Planck-Institute of Neurobiology, Martinsried, Germany.

\section{0 - 2012 Max Planck Institute of Neurobiology Student Research Assistant}

Dr. Irina Dudanova, Prof. Rüdiger Klein, Department of Molecules - Signaling Development, Max-Planck-Institute of Neurobiology, Martinsried, Germany.

\section{0 - 2009 Gymnasium Ottobrunn Allgemeine Hochschulreife (Abitur)}

\section{Publication list}

S. Krinner, T. Butola, S. Jung, C. Wichmann, T. Moser, "RIM-binding protein 2 promotes a large number of Cav1.3 $\mathrm{Ca}^{2+}$-channels and contributes to fast synaptic vesicle replenishment at hair cell active zones", Frontiers in Cellular Neuroscience. (Accepted for publication, October, 2017)

C. Imig, S. Min, S. Krinner, M. Arancillo, C. Rosenmund, T. C. Südhof, J. Rhee, N. Brose, B. H. Cooper, "The Morphological and Molecular Nature of SV Priming at Presynaptic Active Zones", Neuron. 2014 Oct 22;84(2):416-31

S. Weiler, S. Krinner, A. B. Wong, T. Moser \& T. Pangršič, "ATP hydrolysis is critically required for function of Cav1.3 channels in cochlear inner hair cells via fueling $\mathrm{Ca}^{2+}$ clearance", J. Neuroscience. 2014 (34), 6843-8 
